\title{
Topographie, Struktur und Dynamik thermisch aufgedampfter Polymerfilme
}

\author{
Dissertation \\ zur Erlangung des Doktorgrades \\ der Mathematisch-Naturwissenschaftlichen Fakultäten \\ der Georg-August-Universität zu Göttingen
}

vorgelegt von

\section{Christian Vree}

aus Wolfenbüttel

Göttingen, 2009 
D 7

Referent:

Prof. Dr. S. G. Mayr

Korreferent:

Prof. Dr. S. Herminghaus

Tag der mündlichen Prüfung: $\quad$ 06.07.2009 


\section{Inhaltsverzeichnis}

1 Einleitung 1

2 Grundbegriffe der Glas- und Polymerphysik 5

2.1 Dynamik in amorphen Systemen . . . . . . . . . . . . . . 5

2.1.1 Amorphe Materialien und Gläser . . . . . . . . . . . . 5

2.1.2 Relaxationen in Gläsern . . . . . . . . . . . . . . . . . 6

2.2 Grundbegriffe der Polymerphysik . . . . . . . . . . . . . . . . . 7

3 Experimentelle Methoden 11

3.1 Probenherstellung . . . . . . . . . . . . . . . . . . . . . 11

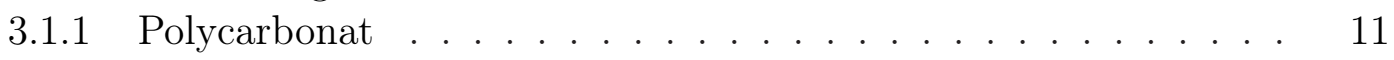

3.1 .2 Thermisches Verdampfen . . . . . . . . . . . . . . . . . . 12

3.1.3 Substrate . . . . . . . . . . . . . . . . . . 12

3.2 Probencharakterisierung . . . . . . . . . . . . . . . 13

3.2.1 Fourier-Transformations-Infrarotspektroskopie zur Analyse der chemischen Zusammensetzung . . . . . . . . . . . 13

3.2.2 Größenausschlusschromatographie und Massenspektrometrie zur Bestimmung der Kettenlänge . . . . . . . . . . . . . . 14

3.2.3 Röntgendiffraktometrie und -reflektometrie . . . . . . . . . . . . 15

3.2.4 Größen zur Charakterisierung von Oberflächen . . . . . . . . . . 16

3.2.5 Rasterkraftmikroskopie . . . . . . . . . . . 18

4 Kontinuumsmodellierung 29

4.1 Grundlagen der Kontinuumsmodellierung . . . . . . . . . . . . . . . . . 29

4.2 Modell zum Schichtwachstum dünner Polymerfilme . . . . . . . . . . . 30

4.2.1 Entwicklung der Kontinuumsgleichung . . . . . . . . . . . . 30

4.2.2 Stabilitätsanalyse der Kontinuumsgleichung . . . . . . . . . . 31

4.2.3 Numerische Lösung der Kontinuumsgleichung . . . . . . . . . . 31

5 Molekulardynamik Simulationen 33

5.1 Grundlagen der MD Simulation . . . . . . . . . . . . . . . . 33

5.2 MD Simulation eines Polymerfilms . . . . . . . . . . . . 34

5.2.1 Das Potential . . . . . . . . . . . . . . . 34

5.2.2 Herstellung und Vorbereitung der Simulationszellen . . . . . . . 36

5.2.3 Simulation einer einzelnen Kette auf der Oberfläche . . . . . . . 39

5.2.4 Simulation des Einflusses der Oberflächen auf die Dynamik . . . 39

5.2.5 Verwendete Computer-Cluster . . . . . . . . . . . . 39 
6 Experimentelle Ergebnisse $\quad 41$

6.1 Struktur thermisch aufgedampfter PC Schichten . . . . . . . . . . . . 41

6.1.1 Zusammensetzung und Struktur . . . . . . . . . . . . . . 41

6.1.2 Kettenlänge und Molmassenverteilung . . . . . . . . . . . . 43

6.2 Topographieentwicklung der thermisch aufgedampften PC Schichten . . $\quad 45$

6.2.1 Bestimmung der Schichtdicke . . . . . . . . . . . . 45

6.2.2 Einfluss des Substrattyps auf die Topographie . . . . . . . . . . 46

6.2.3 Zeitliche Entwicklung der Topographie . . . . . . . . . . . . 56

6.3 Lokale mechanische Eigenschaften . . . . . . . . . . . . . . 57

6.3.1 Messungen mit Kraft-Abstandskurven . . . . . . . . . . . . . . 57

6.3.2 Messungen mit Ultraschall-Kraftmikroskopie . . . . . . . . . . . 58

7 Ergebnisse der Kontinuumsmodellierung $\quad 65$

7.1 Modellierung eines instabilen Systems . . . . . . . . . . . . . . . . 65

7.2 Modellierung eines stabilen Systems . . . . . . . . . . . . . . . 68

8 Ergebnisse der MD Simulationen $\quad 71$

8.1 Amorphe Struktur und Glasübergang . . . . . . . . . . . . . . . 71

8.2 Dynamik einer einzelnen Kette auf der Oberfläche . . . . . . . . . . . 72

8.3 Einfluss der Oberflächen auf die Dynamik . . . . . . . . . . . . . 77

8.3.1 Schwerpunktbewegung . . . . . . . . . . . . . 78

8.3.2 Fluktuationen des Gyrationsradius . . . . . . . . . . . . . 79

9 Diskussion 83

9.1 Struktur der thermisch aufgedampften PC Schichten . . . . . . . . 83

9.1.1 Thermische Zersetzung von PC . . . . . . . . . . . . 83

9.1.2 Thermisches Aufdampfen von PC . . . . . . . . . . . . 86

9.2 Topographieentwicklung . . . . . . . . . . . . . . . . . . . . 89

9.2.1 Einfluss der Grenzflächen auf die Anfangsstadien beim thermischen Aufdampfen . . . . . . . . . . . . . . . . . 89

9.2.2 Zeitliche Entwicklung der Topographie . . . . . . . . . . . . 93

9.3 Lokale mechanische Eigenschaften . . . . . . . . . . . . . . . . . . . . 94

9.4 Diskussion der MD Simulationsergebnisse . . . . . . . . . . . . . . . 96

9.4.1 Betrachtung einer einzelnen Kette auf der Oberfläche . . . . . . 97

9.4.2 Einfluss der Oberflächen auf die diffusive Dynamik . . . . . . . 97

9.4.3 Einfluss der Oberflächen auf die konformative Dynamik . . . . . 100

9.4.4 Kopplung von diffusiver und konformativer Dynamik . . . . . . 104

$\begin{array}{ll}10 \text { Zusammenfassung } & 105\end{array}$

11 Anhang 109

11.1 Hochauflösende Massenspektren . . . . . . . . . . . . . . . . . . . . . . 109

11.2 Exemplarische Kleinwinkelröntgenmessung . . . . . . . . . . . . . . . 110

11.3 Thermisches Verdampfen von Polypropylen und Polystyrol . . . . . . . 111

11.4 Vergleich der Herstellungsvarianten in der MD Simulation . . . . . . . 112

$\begin{array}{ll}\text { Literaturverzeichnis } & 115\end{array}$ 


\section{Abbildungsverzeichnis}

2.1 Glasübergang und potentielle Energielandschaft. . . . . . . . . . . . . . 6

2.2 Schematische Darstellung einer Polymerkette. . . . . . . . . . . . 8

2.3 Beispiel einer mittleren Verschiebungsweite für Polymerketten. . . . . . 9

3.1 Struktur von PC (Bisphenol A-Polycarbonat). . . . . . . . . . . . . . 11

3.2 Prinzip der Größenausschlusschromatographie. . . . . . . . . . . . . . . 14

3.3 Schematischer Aufbau eines konventionellen AFMs und eines AFAMs. . 18

3.4 Schematische Darstellung der Cantilever Auslenkung und Kraft-Verschiebungskurve. . . . . . . . . . . . . . . . . 2 20

3.5 Mechanisches Modell eines AFM Cantilevers. . . . . . . . . . . . . . . 22

3.6 AFAM Messung zur Verdeutlichung der Kontrastumkehr. . . . . . . . . 25

3.7 Erweiterung des MultiMode AFMs zu einem AFAM. . . . . . . . . . 26

5.1 In der MD Simulation verwendete Potentiale. . . . . . . . . . . . 35

5.2 Schematische Darstellung zur Verdeutlichung der vorhandenen Wechselwirkungen. . . . . . . . . . . . . . . . . 36

5.3 Schematische Darstellung der Simulationszelle. . . . . . . . . . . . 38

6.1 Weitwinkelröntgenmessung einer thermisch aufgedampften PC Schicht und Vergleichsmessung aus der Literatur. . . . . . . . . . . . . . .

6.2 FTIR Messung an einer thermisch aufgedampften PC Schicht und Vergleichsspektrum aus der Literatur. . . . . . . . . . . . . . . . . 42

6.3 SEC Messung von thermisch aufgedampftem PC und von PC Granulat. 44

6.4 Massenspektrum von thermisch aufgedampftem PC. . . . . . . . . . . . 44

6.5 Kalibrierung der Schwingquarzwaage. . . . . . . . . . . . . . . 45

6.6 Topographieentwicklung mit der Schichtdicke für PC auf Si. . . . . . . 48

6.7 Rauigkeitsentwicklung für $\mathrm{PC}$ auf Si. . . . . . . . . . . . . . . . . 49

6.8 Korrelationsfunktionen für $\mathrm{PC}$ auf Si. . . . . . . . . . . . . . . . . . 49

6.9 Topographieentwicklung mit der Schichtdicke für $\mathrm{PC}$ auf $\mathrm{SiO}_{2}$. . . . . . $\quad 50$

6.10 Rauigkeitsentwicklung für $\mathrm{PC}$ auf $\mathrm{SiO}_{2} \ldots \ldots \ldots \ldots$. . . . . . . . . . . 51

6.11 Korrelationsfunktionen für $\mathrm{PC}$ auf $\mathrm{SiO}_{2}$. . . . . . . . . . . . 51

6.12 Topographieentwicklung mit der Schichtdicke für PC auf GaAs. . . . . 52

6.13 Rauigkeitsentwicklung für PC auf GaAs. . . . . . . . . . . . . . . . 53

6.14 Korrelationsfunktionen für PC auf GaAs. . . . . . . . . . . . . . 53

6.15 Topographieentwicklung mit der Schichtdicke für PC auf PC. . . . . . . 54

6.16 Rauigkeitsentwicklung für $\mathrm{PC}$ auf PC. . . . . . . . . . . . . . . 55

6.17 Korrelationsfunktionen für PC auf PC . . . . . . . . . . . . . . 55 
6.18 Topographieentwicklung mit der Zeit. . . . . . . . . . . . . 56

6.19 Zeitentwicklung des Anteils der unbedeckten Fläche. . . . . . . . . . . 57

6.20 Übersichtskarten der elastischen Eigenschaften. . . . . . . . . . . . . . 59

6.21 Verformung der Oberfläche durch Indentationsexperimente. . . . . . . . 60

6.22 AFAM Bilder von einer Nickel und einer Eisen-Palladium Schicht. . . . 61

6.23 Vergleich von AFAM Messungen im Spektroskopie-Modus an verschiedenen Materialien. . . . . . . . . . . . . . . . . 62

6.24 Topographie eines PC Substrats vor und nach der AFAM Messung. . . 63

7.1 Topographieentwicklung mit der Schichtdicke für Lösungen des Kontinuumsmodells mit $A>0 \ldots \ldots$. . . . . . . . . . . . 66

7.2 Rauigkeitsentwicklung für Lösungen des Kontinuumsmodells mit $A>0 . \quad 67$

7.3 Korrelationsfunktionen für Lösungen des Kontinuumsmodells mit $A>0 . \quad 67$

7.4 Topographieentwicklung mit der Schichtdicke für Lösungen des Kontinuumsmodells mit $A<0 \ldots \ldots$. . . . . . . . . . . . . . 68

7.5 Rauigkeitsentwicklung für Lösungen des Kontinuumsmodells mit $A<0 . \quad 69$

7.6 Korrelationsfunktionen für Lösungen des Kontinuumsmodells mit $A<0 . \quad 69$

8.1 Glasübergang während des Abkühlens und Paarkorrelationsfunktion. $\quad 72$

8.2 Verlauf der Gesamtenergie, typische Kettenlängenverteilung und Vergleich von Gyrationsradius und End-zu-End Abstand. . . . . . . . . . . 73

8.3 Momentaufnahmen der Zelle mit einer Kette auf der Oberfläche. . . . . 74

8.4 Zeitlicher Verlauf von Gyrationsradius und End-zu-End Abstand. . . . 75

8.5 Vergleich der mittleren Verschiebungsweiten von einer einzelnen Kette mit Zellenmittelwerten bei $T=0,40 \epsilon / \mathrm{k}_{\mathrm{B}}$. . . . . . . . . . . . . . 75

8.6 Vergleich der mittleren Verschiebungsweiten von einer einzelnen Kette mit Zellenmittelwerten bei $T=0,44 \epsilon / \mathrm{k}_{\mathrm{B}} \ldots \ldots \ldots$. . . . . . 76

8.7 Zur Definition des Oberflächen- und des Volumenbereichs in einer Simulationszelle. . . . . . . . . . . . . . . . . . . . . 77

8.8 Effektive Diffusionskonstante in Abhängigkeit von der Tiefe. . . . . . . 78

8.9 Arrhenius-Auftragung der effektiven Diffusionskonstante. . . . . . . . . 79

8.10 Tiefenprofil der Fluktuationsstärke des Gyrationsradius für verschiedene Temperaturen. . . . . . . . . . . . . . 80

8.11 Tiefenprofil der Fluktuationsstärke des Gyrationsradius für verschiedene Beobachtungszeiträume. . . . . . . . . . . . . . .

8.12 Entwicklung der Fluktuationsstärke des Gyrationsradius mit der Länge des Beobachtungszeitraums für verschiedene Temperaturen. . . . . . . .

9.1 Einfluss der Haltezeit bei verschiedenen Temperaturen im Vakuum auf das Molekulargewicht von PC nach Golden und Davis (aus [Dav64]). . 85

9.2 Vergleich von FTIR Messungen an verschieden hergestellten PC Schichten. 86

9.3 Abhängigkeit der Depositionsrate von der Temperatur und der Zeit. . . 88

9.4 Abhängigkeit der Korrelationslänge und des Rauigkeitsexponenten von der Schichtdicke. . . . . . . . . . . . . . . . . . . 92

9.5 Abnahme der Glasübergangstemperatur mit der Schichtdicke und der Molmasse. . . . . . . . . . . . . . . . . . . . . 
9.6 Arrhenius-Auftragung der effektiven Diffusionskonstante zusammen mit Bestimmung der Aktivierungsenthalpie. . . . . . . . . . . . . . .

9.7 Entwicklung der Fluktuationsstärke des Gyrationsradius mit der Länge des Beobachtungszeitraums für verschiedene Temperaturen mit Anpassung nach Gl. (9.4). . . . . . . . . . . . . . . . . . . . . . . 102

9.8 Arrhenius-Auftragung der charakteristischen Relaxationszeit. . . . . . . 103

11.1 Hochaufösendes Massenspektrum von thermisch aufgedampftem PC. . 109 11.2 SAXS Messung an thermisch aufgedampftem PC auf Si. . . . . . . . . 110

11.3 Topographien von thermisch aufgedampften PP und PS. . . . . . . . . 111

11.4 Vergleich der Tiefenprofile der effektiven Diffusionskonstanten. . . . . . 112

11.5 Vergleich der Fluktuationen des Gyrationsradius. . . . . . . . . . . . 113 



\section{Einleitung}

In der heutigen Zeit findet man Polymere in nahezu jeder Form im alltäglichen Leben. So werden synthetische Polymere als Verpackungsmaterial, in Textilien, als Klebstoffe, für Beschichtungen und für vielerlei andere Dinge eingesetzt, während Biopolymere in Form von DNA, Kohlenhydraten und Proteinen für das Leben selbst mitverantwortlich sind. In der Technik werden vor allem die synthetischen Polymere aufgrund ihres geringen Gewichts, der meist hohen Bruchfestigkeit und Zähigkeit und der vielfältig einstellbaren mechanischen und optischen Eigenschaften eingesetzt [Spr91]. Seitdem Shirakawa, Heeger und MacDiarmid leitfähige Polymere entdeckt haben [Chi77], wofür sie im Jahr 2000 auch den Nobelpreis für Chemie erhalten haben, werden Polymere auch in der Elektronik-Branche immer stärker eingesetzt, z. B. in Batterien und seit einiger Zeit auch als Leuchtdioden.

Dabei nehmen dünne Filme im Zuge der Miniaturisierung elektronischer Bauelemente eine immer wichtigere Rolle ein. In diesem Zusammenhang ist auch die Frage nach der Stabilität und nach Strukturbildung von dünnen Polymerfilmen, die beispielsweise als Photolacke in der Lithographie eingesetzt werden, von zunehmender Bedeutung für Technologie und Wissenschaft. Außerdem gilt die selbstorganisierte Strukturbildung als vielversprechende Methode für die Realisierung zukünftiger Nanostrukturen jenseits der Grenzen der herkömmlichen Lithographie [Was05]. Dabei spielen die Eigenschaften der Oberflächen und der Grenzflächen zu den klassischen in der Technik und der Elektronik verwendeten Materialien eine wichtige Rolle. Daher ist das Verständnis der Dynamik von Makromolekülen auf Oberflächen und Grenzflächen eine der grundlegenden Aufgaben der aktuellen Forschung, wie es z. B. von Grest et al. herausgestellt worden ist [Gra03].

Zur Frage der Stabilität gibt es eine Reihe von Untersuchungen zur Entnetzung an dem Modellsystem Polystyrol auf Silizium Substraten [Rei01; See01b; See01a; Bec03], allerdings wird in diesen Studien durchgehend das ,Spin Coating“-Verfahren zur Schichtherstellung verwendet, bei dem das Material mit einem Lösungsmittel aufgetragen wird. Dadurch werden sowohl die Dynamik als auch die Strukturbildung der dünnen Filme stark von den Eigenschaften des Lösungsmittels beeinflusst. Entnetzung bezeichnet meist die Umwandlung eines geschlossenen Films auf einem Substrat in Tröpfchen und trockene Bereiche, wobei viskoses Fließen aufgrund der Grenzflächenenergien als dominanter Mechanismus angenommen wird. Die Dynamik ist durch Druckgradienten im Film getrieben, die hauptsächlich auf der freien Oberflächenenergie des Polymers und dem effektiven Grenzflächenpotential zum Substrat beruhen [Vri66; Sha93; Bec03]. Die Entnetzung kann dabei durch Nukleation oder durch spinodale Entnetzung beginnen, wobei die Einzelheiten noch immer nicht vollständig geklärt sind [Rei03].

Da der Einfluss von Lösungsmitteln bei nasschemischen Herstellungsverfahren wie der „Spin Coating“-Methode eine wesentliche Rolle bei der Dynamik spielt und die Untersuchung der Strukturbildung durch Entnetzung meist eine thermische Nachbe- 


\section{Einleitung}

handlung erfordert, die z. B. die gezielte Herstellung von Nanostrukturen auf Basis der Polymerfilme oder von Polymer-Metall-Multilagen erschwert, werden „trockene“ Herstellungsmethoden als Alternativen benötigt. Hier sind vor allem die Methoden der physikalischen Gasphasenabscheidung (PVD) zu nennen, die im Bereich der Oberflächenphysik und der Beschichtung - insbesondere bei anorganischen Materialien wie Metallen und klassischen Halbleitern - seit langem verwendet werden. Unter physikalischer Gasphasenabscheidung versteht man Beschichtungsmethoden, bei denen das Ausgangsmaterial in die Gasphase überführt wird und auf einem Substrat wieder kondensiert. Man unterscheidet die Methoden nach der Art der Verdampfung, diese kann z. B. thermisch (thermisches Verdampfen), durch einen Elektronenstrahl (Elektronenstrahlverdampfen), durch einen Laser (gepulste Laserdeposition) oder durch Zerstäuben durch Ioneneinschuss (Sputtern) erfolgen. Auch im Bereich der organischen Halbleiter und speziell für organische Leuchtdioden hat sich das thermische Aufdampfen als geeignete Methode etabliert [Bal98; Gef06]. Im Bereich der Polymerphysik sind PVD Methoden nicht so weit verbreitet, auch wenn die gepulste Laserdeposition seit den Arbeiten von Hansen und Robtaille [Han88] für einige bestimmte Polymere verwendet wird [Süs05; Sch06]. Das liegt vor allem daran, dass es neben dem Verdampfungsprozess zu chemischen Veränderungen und zur Reduktion der Molmasse durch Spaltung der Ketten kommen kann [Kub94]. Daher muss der chemische Aufbau nach der Herstellung von Polymerfilmen mit PVD Techniken überprüft werden.

Aufgedampfte Schichten können wie durch „Spin Coating“" hergestellte Schichten als Ausgangspunkt für Entnetzungsexperimente verwendet werden, um z. B. geordnete Nanodots zu erzeugen [Rat07a]. Man kann den Aufdampfprozess selbst aber auch verwenden, um während des Wachstums durch Selbstorganisation bestimmte Strukturen herzustellen. In diesem Zusammenhang müssen die physikalisch relevanten Wachstumsmechanismen und deren Parameter identifiziert werden, was eine große Herausforderung darstellt, insbesondere, da sich das Problem der Strukturbildung während der Deposition als nicht deterministisches Problem erweist. Man kann das Problem im Rahmen von „getriebenen Systemen“ behandeln, welcher bisher für die Molekularstrahlepitaxie (z. B. [Vi191]), das Wachstum amorpher dünner Schichten (z. B. [May99]) oder bei Ionenbestrahlung (z. B. [May01]) verwendet worden ist. Dabei verwendet man stochastische Ratengleichungen zur Beschreibung der Entwicklung des Oberflächenprofils in einem Kontinuumsmodell, um die zugrunde liegenden Mechanismen der Strukturbildung zu bestimmen [Bar95]. Die Kombination von Experimenten und Simulationen mit stochastischen Ratengleichungen ist dabei in der Arbeitsgruppe bereits erfolgreich für andere Systeme angewendet worden [May97; May00; Vau02; Str04; Vre05; Vau07a].

Eine andere Möglichkeit, die Dynamik von Makromolekülen zu untersuchen, bieten Molekulardynamik (MD) Simulationen, die einen Zugang von der mikroskopischen Seite her ermöglichen. Da man in MD Simulationen meist sehr kurze Zeiten betrachtet, wird nicht die Frage nach der Strukturbildung untersucht, da diese meist auf längeren Zeitskalen erfolgt, vielmehr betrachtet man die zugrunde liegende Dynamik eines Systems. Diese ist für Polymere, als Beispiel eines glasbildenden Systems, trotz jahrelanger intensiver Forschung, immer noch nicht gut verstanden. Vor allem sind die molekularen Grundlagen der Relaxationen, Effekte durch geometrische Einschränkungen und wiederum der Einfluss von Oberflächen und Grenzflächen nicht geklärt. Anstatt ein Polymer atomistisch vollständig zu beschreiben, werden in der MD Simulation oft 
vergröberte Modelle eingesetzt, um den kettenartigen Charakter eines Polymers wiederzugeben, ohne den genauen Aufbau eines bestimmten Materials zu berücksichtigen [Doi86; Kre90; Sch03].

Basierend auf Experimenten (darunter vor allem dielektrische Spektroskopie [Lun00]) und auf Simulationen ist es inzwischen etabliert, dass die lokale Dynamik in Gläsern neben struktureller $\alpha$-Relaxation, die dem Fließen zugeordnet wird, mindestens eine sekundäre Relaxation beinhaltet. Die Bewegung der Polymere wird für lange Ketten durch das Modell der Reptation [Gen71] und für kürzere Ketten, deren Kettenlänge unterhalb der Verschlaufungslänge liegt, durch die Rouse-Dynamik [Rou53] beschrieben [Kre90; Kre88]. Im Zusammenhang mit der Dynamik ist auch der Glasübergang für Massivproben untersucht worden [Ben98; Ben99], dessen mikroskopischer Ursprung aber immer noch unklar ist [Hec08]. Simulationen an dünnen Polymerfilmen werden oft als Film zwischen zwei Wänden modelliert [Var02; Bin04; Des07], wodurch die Glasübergangstemperatur je nach Wechselwirkung zwischen Wand und Film erhöht oder verringert sein kann [Tor00]. In Simulationen mit freien Oberflächen findet man eine Verringerung der Glasübergangstemperatur und eine erhöhte Mobilität an der Oberfläche [Tor00; Man91; Pet06; Pet07], die auch bei metallischen Gläsern [Bal96; Böd99; Vau05] und in Experimenten zur Messung der Oberflächendynamik von Polymeren im Glaszustand [Fak07; Fak08] beobachtet worden ist.

In der vorliegenden Arbeit sollen beide Möglichkeiten zur Dynamikuntersuchung, also experimentelle Untersuchungen zusammen mit der Kontinuumsmodellierung und MD Simulationen, verfolgt werden. Zum einen sollen dünne Polycarbonatfilme durch thermisches Aufdampfen hergestellt und anschließend charakterisiert werden. Dazu werden die Bindungsstruktur, also der chemische Aufbau, durch Fourier-Transformations-Infrarotspektroskopie, die Kettenlänge durch Größenausschlusschromatographie und Massenspektrometrie und die Topographie durch Rasterkraftmikroskopie untersucht. Es werden verschiedene Substrate verwendet, um den Einfluss der Grenzfläche überprüfen zu können. Außerdem werden die lokalen mechanischen Eigenschaften mit Hilfe von KraftAbstandskurven und mit akustischer Kraftmikroskopie untersucht. Des Weiteren soll ein minimales Kontinuumsmodell, also eine stochastische Ratengleichung, die so wenig Terme wie möglich beinhaltet, entwickelt werden, das Strukturen in Übereinstimmung mit den Experimenten erzeugt, um die dominierenden physikalischen Prozesse während des Wachstums zu identifizieren. Zum anderen soll mit MD Simulationen an einem (vereinfachten) Polymermodell der Einfluss von freien Oberflächen auf die Dynamik des Systems, vor allem auf die Kinetik des Transports und auf Relaxationen der Konformationen, betrachtet werden. Die Simulationen sollen einen weiten Temperaturbereich um die Glasübergangstemperatur abdecken und in den Auswertungen sollen insbesondere Größen betrachtet werden, die im Prinzip auch experimentell zugänglich sind.

Aufgrund dieser Ziele ist die Arbeit folgendermaßen aufgebaut: Nach einer kurzen Einführung von wichtigen Begriffen aus der Glas- und Polymerphysik in Kapitel 2 werden der Reihe nach die experimentellen Methoden (Kapitel 3), das Kontinuumsmodell (Kapitel 4) und die Molekulardynamik Simulationen (Kapitel 5) vorgestellt. Die Ergebnisse dieser verschiedenen Methoden werden danach in derselben Reihenfolge in den Kapiteln 6-8 präsentiert und in Kapitel 9 gemeinsam diskutiert und in den aktuellen Stand der Forschung eingeordnet. Anschließend wird die Arbeit in Kapitel 10 kurz zusammengefasst. 


\section{Einleitung}

Teile der in dieser Arbeit präsentierten Ergebnisse sind bereits in Fachzeitschriften veröffentlicht. Die experimentellen Untersuchungen der Struktur und der Topographie sind in [Vre08] erschienen. Die Kontinuumsmodellierung und der Vergleich zu den experimentellen Ergebnissen ist in [Vre09b] zu finden und die Molekulardynamik Simulationen zum Einfluss von freien Oberflächen auf die Dynamik sind eingereicht worden [Vre09a]. 


\section{Grundbegriffe der Glas- und Polymerphysik}

In diesem Kapitel sollen einige Grundbegriffe der Polymer- und der Glasphysik behandelt werden. Vor allem geht es um die Definition einiger statistischer Größen der Polymerphysik und grundlegende Eigenschaften von Gläsern und amorphen Materialien.

\subsection{Dynamik in amorphen Systemen}

\subsubsection{Amorphe Materialien und Gläser}

Als amorph bezeichnet man im Allgemeinen Materialien, die keine langreichweitige Ordnung, bzw. Periodizität aufweisen, wie sie einen Kristall auszeichnet [Ell90]. Stattdessen ist die Struktur ungeordnet wie in einer Flüssigkeit, so dass es zwar eine lokale Nahordnung gibt, die aber nicht weiter als einige Atomabstände reicht. Dies sieht man z. B. in der Paarkorrelationsfunktion (auch radiale Verteilungsfunktion RDF $^{1}$ genannt) $g(r)=\left(\frac{V}{N}\right)\left[\frac{n(r)}{4 \pi r^{2} d r}\right]$ mit Volumen $V$, Gesamtteilchenzahl $N$ und mittlerer Teilchenzahl $n(r)$ in der Kugelschale $[r, r+d r]$, die die Anzahl anderer Teilchen im Abstand $r$ eines Teilchens angibt. Somit ist die Paarkorrelationsfunktion ein Maß für die lokale Dichte. Die Paarkorrelationsfunktion kann experimentell in Streuexperimenten über den Strukturfaktor bestimmt werden. Für einen Kristall erwartet man aufgrund der periodischen Anordnung scharfe Maxima in bestimmten periodischen Abständen, wohingegen es in einem idealen Gas keine Dichtefluktuationen gibt und somit in jedem Abstand, der größer als eine Bindungslänge ist, $g(r)=1$ gilt. In amorphen Systemen und Flüssigkeiten findet man ein ausgeprägtes Maximum beim Nächsten-Nachbar Abstand und dann aufgrund der verschwindenden Ordnung Maxima mit stark abnehmender Intensität bei den Übernächsten-Nachbar Abständen, den Über-Übernächsten usw. Bei größeren Entfernungen ist wiederum $g(r)=1 \mathrm{zu}$ erwarten.

Weist das amorphe Material beim Abkühlen zusätzlich noch einen Glasübergang auf, nennt man es auch ein Glas [El190]. Beim Glasübergang ändern sich die Ableitungen thermodynamischer Größen, wie der Wärmekapazität oder der thermische Ausdehnungskoeffizient, mehr oder weniger abrupt von festkörperartig zu flüssigkeitsähnlich. Durch Messung des Volumens in Abhängigkeit von der Temperatur kann demnach die Glasübergangstemperatur $T_{g}$ bestimmt werden. Schematisch ist eine solche Messung in Abb. 2.1(a) gezeigt (aus [Deb01]). Um zu verhindern, dass das Material beim Abkühlen in den kristallinen Zustand übergeht, muss man die Kristallisation kinetisch hemmen,

\footnotetext{
${ }^{1}$ RDF für Radial Distribution Function.
} 

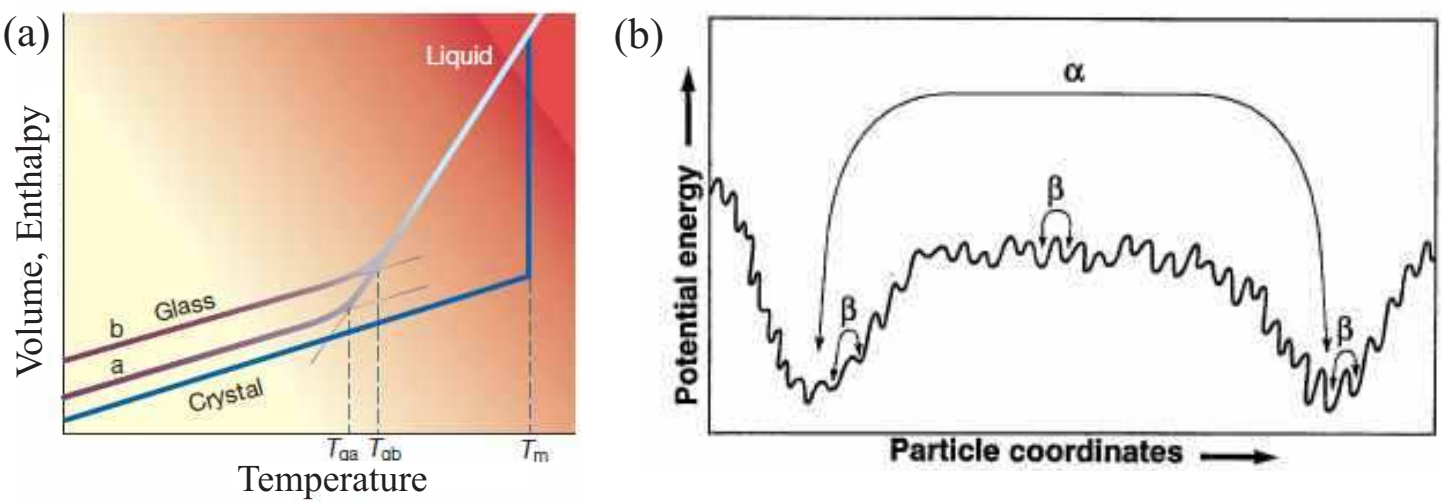

Abbildung 2.1: (a) Schematische Darstellung der Volumenänderung mit der Temperatur eines glasbildenden Systems (aus [Deb01]). Im Bereich der Glasübergangstemperatur ändert sich die Steigung deutlich. (b) Schematische Darstellung einer potentiellen Energielandschaft (PEL), in der Übergänge zwischen Minima durch $\alpha$ - und $\beta$-Prozesse stattfinden können (aus [Sti95]).

also schnell abkühlen, um die Struktur der Schmelze einzufrieren. Dabei ist die Glasübergangstemperatur abhängig von der Abkühlrate. Die statischen Strukturen von Glas und Flüssigkeit sind kaum zu unterscheiden, während die Dynamik sehr verschieden ist. Aufgrund der bestehenden Unordnung gibt es im Gegensatz zum Kristall sehr viele verschiedene Glaszustände, die energetisch gleich sein oder sich energetisch unterscheiden können. Diese Möglichkeiten der Konfigurationen kann man nach Stillinger im Bild einer potentiellen Energielandschaft (PEL) veranschaulichen (vgl. Abb. 2.1(b)), indem man die möglichen Konfigurationen auf eine Achse projiziert und darüber die zugehörigen potentiellen Energien aufträgt. So existieren unterschiedliche Minima, die durch Energiebarrieren getrennt sind. Neben diesen lokalen Minima gibt es je nach Fragilität des Materials eine Überstruktur und es bilden sich verschieden tiefe Energiemulden, die sog. „Megabasins“, wie es in den Abb. 2.1(b) angedeutet ist. Durch verschiedene Relaxationsprozesse, die im folgenden Abschnitt kurz beschrieben werden, kann ein Glas seine Konfiguration und damit die Lage in der PEL ändern.

Eine ausführliche Einführung in das Themengebiet der amorphen Materialien bietet z. B. das Buch von Elliott [Ell90].

\subsubsection{Relaxationen in Gläsern}

In Gläsern gibt es eine Vielzahl von Relaxationsprozessen, die mit verschiedenen Frequenzen ablaufen [Lun00]. Bis heute gibt es keine Theorie und vor allem keine mikroskopische Erklärung, die alle Prozesse beschreiben und erklären kann, obwohl es eine Reihe verschiedener Ansätze gibt (die Adams-Gibbs Theorie [Ada65], das freie Volumen Modell [Coh59], u. a.). Mit dielektrischer Spektroskopie konnte z. B. an Glyzerin und Polypropylencarbonat gezeigt werden, dass das Relaxationsspektrum von hochfrequenten Molekülschwingungen im infraroten Bereich, über den „Boson-Peak“ und zusätzliche schnelle Prozesse (,fast process"), die von mehreren Theorien vorausgesagt 
wurden [Nga88; Göt92], hin zu sekundären Relaxationen reicht, die z. B. als „excess Wing" [Dix90], Johari Goldstein $\beta$ [Joh70], als extra Maximum oder als Schulter auftreten können [Sch00; Lun00], bis schließlich die primäre oder $\alpha$-Relaxation auftritt, die strukturelle Relaxationen und damit den Übergang zum viskosen Fließen beinhaltet. Im Bild der PEL wird die $\alpha$-Relaxation dem Übergang zwischen verschiedenen Megabasins zugeordnet, während die $\beta$-Relaxationen Übergängen zwischen benachbarten Minima entspricht und damit durch lokale Anregungen, bzw. Änderungen und Umordnungen, erklärt werden kann [Sti95]. Dies ist in Abb. 2.1(b) veranschaulicht.

Bei Polymeren können aufgrund der chemischen und strukturellen Komplexität zusätzliche Relaxationen, wie z. B. Seitengruppenbewegungen, oder Effekte durch Verschlaufungen der Ketten entstehen.

\subsection{Grundbegriffe der Polymerphysik}

An dieser Stelle soll keine ausführliche Einführung in die Physik von Polymeren und Makromolekülen folgen, diese findet man z. B. in den Büchern von Sperling [Spe85] oder Doi und Edwards [Doi86]. Stattdessen sollen einige grundlegende Definitionen gegeben werden.

Ein Polymer ist eine Kette aus sich wiederholenden Grundbausteinen, den Monomeren, die meist aus einfachen organischen Kohlenstoffverbindungen bestehen. Diese können entweder linear oder verzweigt vorliegen. Die Eigenschaften eines Polymers sind weniger durch den mikroskopischen Aufbau der Monomere als vielmehr durch die Größe und Flexibilität der Kette und deren Wechselwirkung bestimmt. In einer einfachen Näherung kann man den mikroskopischen Aufbau der Monomere vernachlässigen und sie wie in der Simulation geschehen - durch Kugeln ersetzen, um allgemeine Eigenschaften von Makromolekülen zu untersuchen. Spezifische Eigenschaften bestimmter Polymere können so natürlich nicht betrachtet werden.

Um eine Polymerkette zu beschreiben, benötigt man zum einen statische Größen zur Beschreibung der Länge und damit der Masse der Kette und deren Ausdehnung und zum anderen dynamische Größen zur Beschreibung der Bewegung und Dynamik. Um die Länge, bzw. Größe einer Kette anzugeben, verwendet man experimentell häufig Molmassen und Molmassenverteilungen, während man in Simulationen die Zahl der Bausteine angeben kann. Bei der Angabe der Molmasse unterscheidet man das zahlengemittelte Molekulargewicht $M_{n}$ und das gewichtsgemittelte Molekulargewicht $M_{w}$, die folgendermaßen definiert sind [Doi86]:

$$
\begin{gathered}
M_{n}=\frac{\sum_{i} N_{i} M_{i}}{\sum_{i} N_{i}} \\
M_{w}=\frac{\sum_{i} N_{i} M_{i}^{2}}{\sum_{i} N_{i} M_{i}}
\end{gathered}
$$

Dabei bezeichnet $N_{i}$ die Anzahl der Moleküle mit Molekulargewicht $M_{i}$.

Abb. 2.2 zeigt eine mögliche Konformation einer Polymerkette. Um die räumliche Ausdehnung einer Kette zu beschreiben, werden der End-zu-End Abstand $R_{E}$ und der Gyrationsradius $R_{G}$, der den mittleren Abstand der Monomere vom Schwerpunkt misst, 


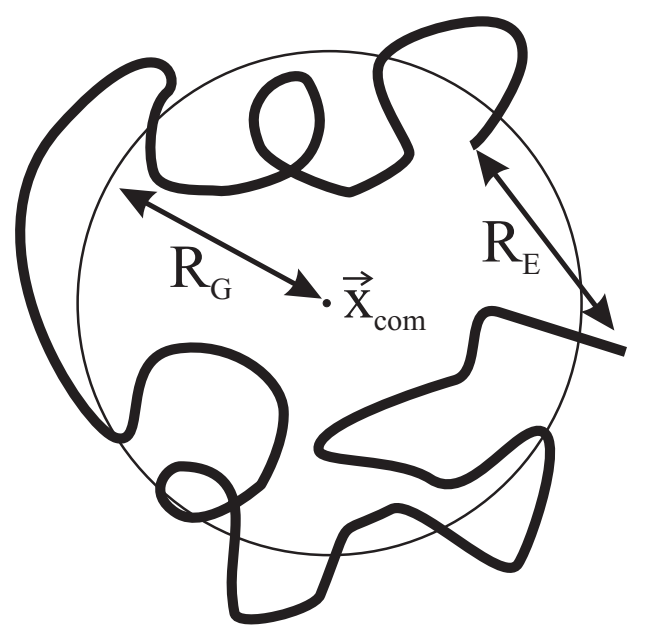

Abbildung 2.2: Schematische Darstellung einer Polymerkette zur Veranschaulichung des Gyrationsradius $R_{G}$ und des End-zu-End Abstands $R_{E}$.

definiert, wobei bei beiden Größen eigentlich nur der Betrag von Interesse ist. Daher werden im Allgemeinen die Quadrate der beiden Größen angegeben. Betrachtet man eine Kette aus $N$ Monomeren an den Positionen $\vec{x}_{i}$ mit $i=1 . . N$ mit dem Kettenschwerpunkt bei $\vec{x}_{\text {com }}$, lassen sich diese wie folgt berechnen:

$$
\begin{aligned}
& R_{E}^{2}=\left(\sum_{i=1}^{N-1} \vec{x}_{i+1}-\vec{x}_{i}\right)^{2} \\
& R_{G}^{2}=\frac{1}{N} \sum_{i=1}^{N}\left(\vec{x}_{i}-\vec{x}_{c o m}\right)^{2}
\end{aligned}
$$

Für lange ungestörte Ketten gilt für die statistischen Mittelwerte vieler Ketten die Beziehung $R_{G}^{2}=\frac{1}{6} R_{E}^{2}$.

Diese Eigenschaften werden aus den Positionen zu einer Zeit $t$ bestimmt und werden daher hier als statische Eigenschaften bezeichnet, dennoch können sie sich natürlich mit der Zeit ändern. Dagegen werden Größen, die aus der Zeitentwicklung z. B. der Positionen bestimmt werden, als dynamische Größen bezeichnet. Ein übliches Maß für die Dynamik ist die mittlere Verschiebungsweite $\mathrm{MSD}^{2}$, die man für alle Monomere, die mittleren Monomere einer Kette oder die Kettenschwerpunkte berechnen kann. Aus der MSD kann im Limes großer Zeiten der Diffusionskoeffizient nach $D=\lim _{t \rightarrow \infty} \frac{(\vec{x}(t)-\vec{x}(0))^{2}}{6 t}$ bestimmt werden [Kre90]. In Abb. 2.3(a) ist ein Beispiel für die Zeitentwicklung der MSD für die mittleren Monomere einer Kette $g_{1}(t)$ und für die Schwerpunkte $g_{3}(t)$ aus der Literatur aufgetragen [Var02], wobei die Kettenlänge unterhalb der sog. „entanglement“Länge $N_{e}$, also der Verschlaufungslänge, ist. D. h., dass Effekte durch Verschlaufungen bei dieser Kettenlänge noch keine Rolle spielen und die Dynamik der Kette durch das Rouse Modell [Rou53] beschrieben werden kann. In dieser Arbeit werden hauptsächlich Kettenlängen unterhalb von $N_{e}$ betrachtet. Auf sehr kurzen Zeitskalen verhält sich das

\footnotetext{
${ }^{2}$ MSD für Mean Square Displacement.
} 
(a)

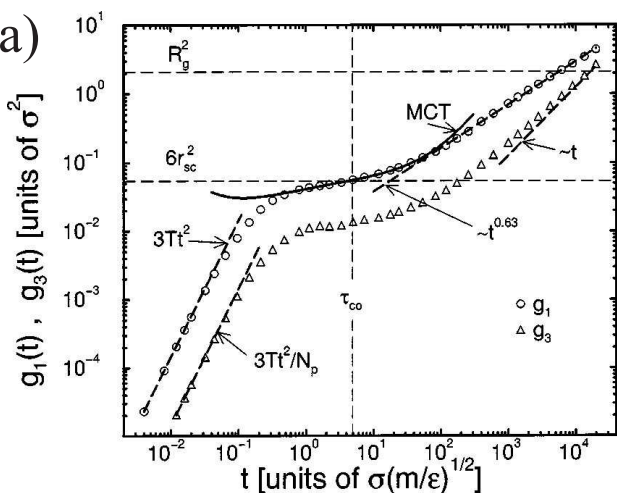

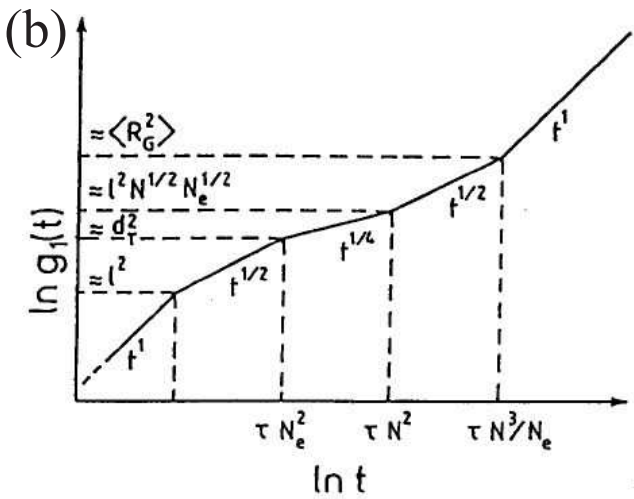

Abbildung 2.3: Beispiel der Zeitentwicklung der mittleren Verschiebungsweite der inneren Monomere $g_{1}(t)$ und der Schwerpunkte $g_{3}(t)$ von Ketten: (a) für Kettenlängen unterhalb der Verschlaufungslänge (Rouse-Dynamik) (aus [Var02]) und (b) für Ketten mit Längen oberhalb der Verschlaufungslänge (Reptations-Dynamik) (aus [Kre90]). In (a) erkennt man, dass die Schwerpunktbewegung das diffusive Regime $(\propto t)$ früher erreicht als die inneren Monomere, da sich für diese ein subdiffusvier Bereich $\left(\propto t^{0,63}\right)$ ausbildet. In (b) sieht man, dass sich für längere Ketten zusätzliche Bereiche mit unterschiedlicher Dynamik ausbilden.

System ballistisch wie bei freien Teilchen. Anschließend folgt ein Plateau- oder Übergangsbereich, in dem jedes Teilchen durch die Anwesenheit der Nachbarn gefangen ist. Es sollte nun direkt der diffusive Bereich folgen (für den $\Delta \vec{x}^{2} \propto t$ gilt), wie man es bei metallischen Gläsern (z. B. [Gue00; Fau03]) findet. Für die MSD der Schwerpunkte $g_{3}(t)$ wird dieses Verhalten auch gefunden, für die MSD der mittleren Monomere $g_{1}(t)$ bildet sich dagegen in den Simulationen ein subdiffusiver Bereich, der durch den Zusammenhalt der Monomere in einer Kette erklärt wird, die sich durch den Zusammenhalt bei der Bewegung gegenseitig behindern. In diesem Bereich kann der Verlauf durch ein Potenzgesetz $g_{1}(t) \propto t^{x}$ mit $x=0,63$ beschrieben werden [Var02]. Erst, wenn sich die Monomere im Mittel weiter als $R_{E}^{2}$ bewegt haben, ist der diffusive Bereich erreicht. Bei längeren Ketten entstehen Verschlaufungen und die Ketten grenzen ihre Bewegungsmöglichkeiten weiter ein. Ihre Dynamik wird durch das Reptationsmodell beschrieben [Gen71]. Eine Kette kann sich nur entlang einer schmalen Röhre, die durch die umliegenden Ketten gebildet wird, bewegen. So entstehen Bereiche, in denen verschiedene Potenzgesetze $\Delta \vec{x}^{2} \propto t^{x}$ mit unterschiedlichen $x$ gelten, wie sie in Abb. 2.3(b) aus [Kre90] aufgetragen sind. Erst wenn eine Kette sich aus dieser Röhre heraus bewegt hat, folgt der eigentliche diffusive Bereich.

Es bietet sich daher für die Auswertung der Dynamik von Kettenmolekülen an, die Schwerpunktbewegung zu betrachten, da man für diese den diffusiven Bereich deutlich früher erreicht. Die mittlere Verschiebungsweite des Schwerpunktes der Kette $i$ ist folgendermaßen definiert:

$$
\Delta \vec{x}_{c o m, i}^{2}(t)=\left(\vec{x}_{c o m, i}(t)-\vec{x}_{c o m, i}\left(t_{0}\right)\right)^{2}
$$

Und damit wird die effektive Diffusionskonstante der Kette $i$ aus dem Langzeitver- 
halten $t \gg t_{0}$ bestimmt:

$$
D_{\text {com }, i}=\Delta \vec{x}_{c o m}^{2}(t) / 6 t
$$

Eine Mittelung (gekennzeichnet durch \langle\rangle ) bei einer bestimmten Temperatur kann nun über alle Ketten erfolgen oder, um den Einfluss der Oberflächen zu untersuchen, durch Einteilung der Zelle in z-Richtung in Intervalle (hier der Breite 0,5 $\sigma$ ), in denen über alle darin vorhandenen Ketten gemittelt wird. Durch eine solche Mittelung erhält man dann eine z-abhängige effektive Diffusionskonstante:

$$
D_{\text {com }}(z)=\left\langle D_{i}\right\rangle_{x_{c o m, z, i} \in[z-0,25, z+0,25]}
$$

Eine andere Möglichkeit die (konformative) Dynamik des Systems zu betrachten, ergibt sich aus den zeitlichen Fluktuationen des Gyrationsradius und des End-zu-End Abstands. Abb. 2.2 verdeutlicht, dass eine größere Änderung des Gyrationsradius immer mit einer Konformationsänderung der gesamten Kette einhergeht. Änderungen des Endzu-End Abstandes können dagegen stärker von Fluktuationen beeinflusst sein, die keine starke Änderung der Konformation bewirken, da eine kleine Änderung am Kettenende einen großen Einfluss auf $R_{E}$ haben kann. Daher werden in dieser Arbeit hauptsächlich die Fluktuationen von $R_{G}^{2}$ betrachtet:

$$
\Delta R_{G, i}^{2}(t)=\sqrt{\frac{1}{n-1} \sum_{\theta=0}^{t}\left(R_{G, i}^{2}(\theta)-\left\langle R_{G, i}^{2}\right\rangle_{t}\right)^{2}}
$$

Dabei bezeichnet $\left\langle R_{G, i}^{2}\right\rangle_{t}$ den zeitlichen Mittelwert des Gyrationsradius der Kette $i$, wenn man zur Mittelung $n$ Momentaufnahmen in der Zeit $t$ heranzieht. Die so berechnete Fluktuationsstärke kann man dann wiederum wie den effektiven Diffusionskoeffizienten z-abhängig mitteln. 


\section{Experimentelle Methoden}

Ein wesentlicher Teil dieser Arbeit ist die Untersuchung der Eigenschaften von dünnen aufgedampften Polymerschichten anhand eines Polycarbonats. Dabei stehen zum einen die Strukturbildung auf verschiedenen Substraten und zum anderen die strukturelle und chemische Charakterisierung des aufgedampften Materials im Zentrum. Ein weiterer Punkt ist die Untersuchung lokaler mechanischer Eigenschaften und damit verbunden der Aufbau eines Ultraschall-Kraftmikroskops (AFAM $\left.{ }^{1}\right)$.

Daher werden in diesem Kapitel zunächst die Grundlagen der Probenherstellung erläutert. Anschließend folgt eine kurze Übersicht über die verwendeten Charakterisierungsmethoden, beginnend mit der Analyse der chemischen Struktur und der Kettenlänge und gefolgt von der Oberflächencharakterisierung. Am Ende des Kapitels werden kurz Möglichkeiten der Messung lokaler mechanischer Eigenschaften mit dem Rasterkraftmikroskop und damit verbunden das Ultraschall-Kraftmikroskop vorgestellt.

\subsection{Probenherstellung}

\subsubsection{Polycarbonat}

Polycarbonat (PC) ist die Bezeichnung für eine Gruppe von synthetischen Polymeren aus der Familie der Polyester. Es wurde erstmals 1953 von H. Schnell hergestellt [Sch56], gehört zu den thermoplastischen Kunststoffen und wird unter verschiedenen Markennamen geführt, z. B. Makrolon (Bayer), Anjalon (J\&A Plastics), Lexan (General Electronics) und CALIBRE Polycarbonate Resins (Dow Chemical). In dieser Arbeit wird das Polycarbonat Bisphenol A-Polycarbonat, dessen Struktur in Abb. 3.1 dargestellt ist, untersucht, für das im Folgenden aber auch der allgemeine Ausdruck Polycarbonat oder kurz PC verwendet wird. PC findet aufgrund der hohen Wärmebeständigkeit, der guten Lichtdurchlässigkeit und vor allem wegen der hohen Schlagzähigkeit in vielen Bereichen, wie z. B. bei Schutzhelmen oder CDs, Anwendung, obwohl es im Vergleich zu anderen Kunststoffen aufwendig und teuer in der Produktion ist.

PC liegt meist in einer amorphen Phase vor und kristallisiert nur unter bestimmten Bedingungen, wie z. B. beim Abkühlen einer übersättigten Lösung [Sch67]. Die

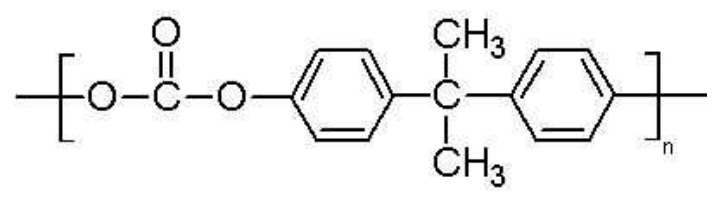

Abbildung 3.1: Struktur von PC (Bisphenol A-Polycarbonat).

\footnotetext{
${ }^{1}$ AFAM für Atomic Force Acoustic Microscope.
} 
Schmelztemperatur von $\mathrm{PC}$ beträgt $T_{m} \approx 225^{\circ} \mathrm{C}$ und die Glasübergangstemperatur etwa $T_{g} \approx 150{ }^{\circ} \mathrm{C}$. Das Monomer hat eine Masse von $M_{w, \text { monomer }}=254 \mathrm{~g} / \mathrm{mol}$ [Mar99]. Die Polymerkette besteht entlang der Hauptkette aus C-C (346 kJ/mol) und C-O $(358 \mathrm{~kJ} / \mathrm{mol})$ Bindungen [Lee64], die beide eine ähnliche Bindungsenergie aufweisen. In dieser Arbeit wird Polycarbonat in Form von farblosem Granulat ${ }^{2}$ mit einem Molekulargewicht von $M_{w} \approx 29000 \mathrm{~g} / \mathrm{mol}$, bezogen von der Firma Sigma-Aldrich ${ }^{3}$, zum Aufdampfen verwendet.

\subsubsection{Thermisches Verdampfen}

Für die Probenherstellung wird ein Verdampfer des Typs LTE ${ }^{4} 11000 \mathrm{~K}$ der Firma Lesker verwendet. Dieser zeichnet sich durch eine sehr stabile Temperaturkontrolle aus, die bereits bei niedrigen Temperaturen stabil regelt, da er für die Herstellung von organischen Schichten entwickelt worden ist und diese sehr empfindlich auf zu hohe Temperaturen reagieren können. Der Verdampfer arbeitet stabil in einem Temperaturbereich von $50{ }^{\circ} \mathrm{C} \leq T \leq 600{ }^{\circ} \mathrm{C}$.

Das Material wird aus einem Aluminiumoxid Tiegel, der max. $1 \mathrm{~cm}^{3}$ Material fasst, in einer Vakuumanlage mit einem Basisdruck von $p \approx 2 \times 10^{-7}$ mbar verdampft. Um den Tiegel zu befüllen, muss die Anlage belüftet werden. Während des Verdampfungsvorgangs wird die Schichtdicke mit einem Schwingquarz überwacht, der mit Hilfe von Ellipsometrie und Kleinwinkelröntgenstreuung (vgl. Abs. 3.2.3) kalibriert ist. Wenn nicht anders angegeben, werden die Schichten bei einer Tiegeltemperatur von $300 \pm 1{ }^{\circ} \mathrm{C}$ auf Substraten mit Raumtemperatur hergestellt. Unter diesen Bedingungen stellt sich mit Polycarbonat zu Beginn eine typische Aufdampfrate von $3 \AA / \mathrm{min}$ ein, die sich über längere Zeit zu $1 \AA /$ min verringert. So erreicht man eine maximale Schichtdicke von etwa $200 \mathrm{~nm}$, ohne den Tiegel nachzufüllen und somit den Aufdampfvorgang und das Vakuum zu unterbrechen.

Neben Polycarbonat bei $T=300{ }^{\circ} \mathrm{C}$ sind testweise auch Polycarbonat bei einer höheren Temperatur on $T=400{ }^{\circ} \mathrm{C}$, Polypropylen bei $T=250{ }^{\circ} \mathrm{C}$ und Polystyrol bei $T=280{ }^{\circ} \mathrm{C}$ verdampft worden. Die Ergebnisse für diese Schichten sind im Anhang in Abs. $11.3 \mathrm{zu}$ finden.

\subsubsection{Substrate}

Um den Einfluss der Grenzfläche zum Substrat auf das Wachstum untersuchen zu können, werden Schichten auf verschiedenen Substraten abgeschieden. Dabei kommen Si(100) Substrate (im Folgenden meist nur Si) mit einer etwa 2 nm dicken natürlichen Oxidschicht [Sou89], thermisch oxidiertes Silizium $\left(\mathrm{SiO}_{2}\right)$ mit einer ca. $500 \mathrm{~nm}$ dicken amorphen Oxidschicht, GaAs(100) (im Folgenden nur GaAs) und Polycarbonat zum Einsatz. Die anorganischen Substrate werden vor dem Einbau in die Vakuumanlage der folgenden Reinigungsprozedur unterzogen:

- $\mathrm{CO}_{2}$-Schneestrahlen

\footnotetext{
${ }^{2}$ Korngröße etwa $2 \mathrm{~mm}^{3}$.

${ }^{3}$ CAS Number 25037-45-0.

${ }^{4}$ LTE für Low Temperature Evaporator.
} 
- 5 min Ultraschallbad mit Aceton

- 5 min Ultraschallbad mit Isopropanol

- Abblasen mit einem Stickstoffgas-Strahl

Das PC Substrat wird durch das ,Spin Coating“-Verfahren auf einem $\mathrm{SiO}_{2}$ Substrat hergestellt. Dazu wird Polycarbonat in einer Mischung aus Chloroform und o-Dichlorbenzol gelöst ${ }^{5}$ und bei 2000 RPM $^{6}$ für 180 s in einem Spin Coater des Typs Delta 20BM der Firma SUSS MicroTech Lithography GmbH aufgeschleudert. Die daraus resultierenden Filme sind ca. $70 \mathrm{~nm}$ dick und sehr glatt ${ }^{7}$, wodurch sie sich gut als Substrat eignen.

\subsection{Probencharakterisierung}

\subsubsection{Fourier-Transformations-Infrarotspektroskopie zur Analyse der chemischen Zusammensetzung}

Da Schwingungsfrequenzen von Molekülbindungen meist im infraroten (IR) Bereich liegen, kann man IR-Strahlung nutzen, um Bindungen durch Absorption der Strahlung zu Schwingungen anzuregen. Somit erlauben das Absorptions- und Transmissionsverhalten, Informationen über den molekularen Aufbau bzw. die chemischen Bindungsverhältnisse zu gewinnen, da ein Photon genau dann absorbiert wird, wenn seine Frequenz und damit seine Energie mit der Schwingungsfrequenz des Moleküls übereinstimmt. So kommt es zu molekülspezifischen Absorptionskanten, die durch Vergleiche mit Literaturdaten (z. B. in [Fal97; Sch64]) identifiziert werden können. Eine detaillierte Einführung zur IR-Spektroskopie findet man z. B. in den Büchern von Kruse und Nakamoto [Kru62; Nak63].

Bei der Fourier-Transformations-Infrarotspektroskopie (FTIR)-Methode wird die Probe gleichzeitig mit allen Wellenlängen des verfügbaren Bereichs bestrahlt und das resultierende Spektrum anschließend durch Fourier-Transformation in die einzelnen Wellenlängen zerlegt. So wird eine hohe Aufösung bei geringer Messzeit erreicht.

Die FTIR Messungen in dieser Arbeit sind in Zusammenarbeit mit dem Institut für Materialphysik, Universität Göttingen, mit einem Bruker Tensor 37 mit MCT-Detektor durchgeführt worden. Die Aufösung beträgt $4 \mathrm{~cm}^{-1}$, für ein Spektrum wird über 50 Messungen gemittelt und die Spektren werden um eine Hintergrundmessung korrigiert. Um störende Wassersignale zu minimieren, wird der Probenraum mit getrockneter Luft gespült. Als Substrat eignet sich raues Aluminium, um schichtdickenabhängige Interferenzen zu vermeiden.

\footnotetext{
${ }^{5}$ Mischungsverhältnis der Lösungsmittel 1:1; darin wird $1 \%$ Massenanteil Polycarbonat gelöst.

${ }^{6} \mathrm{RPM}$ für Revolutions Per Minute.

${ }^{7}$ RMS-Rauigkeit $\sigma \leq 0,3 \mathrm{~nm}$ - siehe Gl. (3.2), bestimmt aus AFM Messungen (vgl. Abs. 3.2.5).
} 


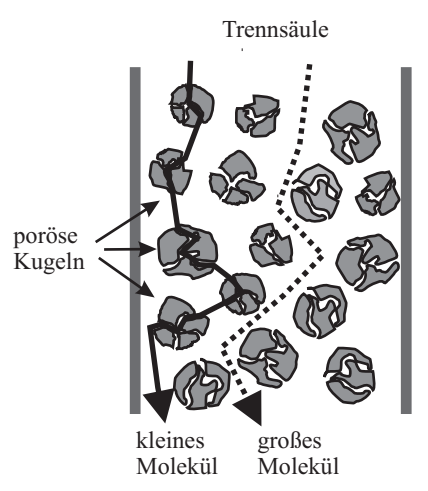

Abbildung 3.2: Prinzip der Größenausschlusschromatographie: Kleinere Moleküle können in mehr Poren diffundieren und so ihre Zeit in der Messsäule erhöhen. Die Moleküle werden ihrer Größe entsprechend getrennt.

\subsubsection{Größenausschlusschromatographie und Massenspektrometrie zur Bestimmung der Kettenlänge}

Neben den Bindungsverhältnissen in einem Polymer, die man mittels FTIR bestimmen kann, ist die Molmasse und damit die Kettenlänge ein wichtiges Charakteristikum. Ein gängiges Verfahren ist die Größenausschlusschromatographie $\left(\mathrm{SEC}^{8}\right)$, bei der das zu untersuchende Material in Lösung gebracht und durch ein System aus Trennsäulen gepumpt wird (vgl. z. B. [Wil70]). Die Trennsäulen beinhalten ein Trenngel, das aus hochporösen Kugeln mit einigen $\mu \mathrm{m}$ Radius und verschieden großen Porendurchmessern besteht. Die Moleküle werden aufgrund ihres hydrodynamischen Radius aufgetrennt, da kleineren Molekülen mehr Poren zur Verfügung stehen, in die sie diffundieren können, wie es in Abb. 3.2 skizziert ist. Hinter der Säule wird z. B. durch Messung der Brechungsindizes die Konzentration als Funktion der Zeit detektiert.

Um aus der Zeit auf die Molekülmasse zu schließen, müssen geeignete Polymerstandards zur Kalibrierung verwendet werden. Idealerweise verwendet man dazu Standards mit niedriger Polydispersität des zu untersuchenden Materials. Man kann die Kalibrierung auch universell mit einem anderen Material als Standard durchführen („Prinzip der universellen Kalibrierung" [Wil70]), dann können die berechneten absoluten Molmassen aber stark von den tatsächlichen abweichen und nur noch Verhältnisaussagen zulassen.

Die SEC Messungen an PC sind in Zusammenarbeit mit B. Steisel (AG Prof. Buback) aus dem Institut für Physikalische Chemie, Universität Göttingen, an einem WatersSystem mit drei PSS-SDV-Säulen mit nominellen Porendurchmessern von $10^{5}, 10^{3}$ und $10^{2} \AA$ bei einer Partikelgröße von $5 \mu \mathrm{m}$ mit Tetrahydrofuran (THF) als Eluent und einer Flussgeschwindigkeit von $1 \mathrm{ml} /$ min durchgeführt worden, das mit Polystyrol (PS)Standards kalibriert ist.

Um überhaupt ein Signal zu erhalten, benötigt man mindestens $2 \mathrm{mg}$ Material gelöst in THF, was aufgrund der hier verwendeten langsamen Aufdampfrate (vgl. Abs. 3.1.2) ein Problem ist. Daher wird für die SEC Proben nicht auf typische Substrate, sondern auf eine ca. $11 \times 11 \mathrm{~cm}^{2}$ große Edelstahlfolie in zwei Etappen über insgesamt 4 Tage gedampft. So erhält man eine etwa $330 \mathrm{~nm}$ dicke PC Schicht, bzw. etwa $5 \mathrm{mg}$ Material.

\footnotetext{
${ }^{8}$ SEC für Size Exclusion Chromatography.
} 
Die Folie wird anschließend zerschnitten und das Material in THF abgelöst.

Neben der Größenausschlusschromatographie wird Massenspektrometrie verwendet, um die Molmasse genauer zu bestimmen. In Zusammenarbeit mit F. Günzler (AG Prof. Buback) aus dem Institut für Physikalische Chemie, Universität Göttingen, sind dieselben Proben, die auch bei den SEC Messungen zum Einsatz kamen, an einem Elektrospray-Ionisations (ESI) Massenspektrometer ${ }^{9}$ und anschließend hochauflösend an einem FT-ICR-Massenspektrometer (7 Tesla APEX IV) mit einer externen ESI Ionenquelle (APOLLO), einer Ionentransferoptik und einer ICR-Ionenfalle (INFINITY) von Bruker Daltonics vermessen worden. Beide Geräte können Masse-zu-Ladungs Verhältnisse im Bereich $100 \mathrm{u} \leq m / z \leq 2000 \mathrm{u}$ detektieren. Die Ionisierung kann dabei durch Natriumionen (Masse $+23 \mathrm{u}$ pro ioniesierendes Natriumatom), Ammoniumionen $(+18 \mathrm{u})$ und Protonen $(+1 \mathrm{u})$ erfolgen, wobei Natriumionisierung bei den nicht hochaufgelösten Massenspektren bevorzugt auftreten. Es können Einfachionisierungen $(z=1)$ und Mehrfachionisierungen $(z \geq 2)$ auftreten, wobei die Zahl $z$ den Grad der Ionisierung angibt. Als Lösungsmittel werden THF für die normale und MethanolAmmoniumacetat (MeOh + NH4OAc) für das FT-ICR-MS verwendet. Eine genauere Beschreibung ist z. B. in [Bub07] zu finden.

\subsubsection{Röntgendiffraktometrie und -reflektometrie}

Röntgenmethoden eignen sich je nach Aufbau, bzw. Einfallswinkel, zur Strukturanalyse (Weitwinkel-Streuung) [Ash01] und zur Untersuchung der Schichtdicke und Rauigkeit von dünnen Filmen und Grenzflächen (Kleinwinkel-Streuung) [Kie31]. Für alle Röntgenmessungen dieser Arbeit wird ein Bruker AXS D8 Diffraktometer in BraggBrentano-Geometrie ( $\theta / 2 \theta$-Geometrie) mit einer $\mathrm{Cu}$-Röhre mit charakteristischer Wellenlänge $\lambda_{C u-K_{\alpha}}=0,154 \mathrm{~nm}$ (als gewichtetes Mittel aus den Wellenlängen der $\mathrm{Cu}-\mathrm{K}_{\alpha 1}$ und $\mathrm{Cu}-\mathrm{K}_{\alpha 2}$ Linien) verwendet.

Bei Kleinwinkel-Streuexperimenten misst man typischerweise unter Winkeln im Bereich $2 \theta \leq 6^{\circ}$, wobei es bei sehr kleinen Winkeln unterhalb eines kritischen Winkels $\theta_{c}$ zur Totalreflexion kommt, da Materie für Röntgenstrahlen optisch dünner als Luft ist. Oberhalb von $\theta_{c}$ dringt ein Teil der Strahlung in die Probe ein und wird dann an Grenzflächen von Medien mit unterschiedlichem Brechungsindizes, also z. B. am Substrat und an der Oberfläche reflektiert ${ }^{10}$, wodurch es zu winkelabhängigen Schichtdickeninterferenzen kommt, aus denen man die Schichtdicke $d$ bestimmen kann, da die Strahlen dann konstruktiv interferieren, wenn die Bragg-Bedingung erfüllt ist: $2 d \sin \theta=n \lambda$, wobei $n$ die Ordnung eines Maximums bezeichnet ${ }^{11}$. Da kleine Winkel betrachtet werden, gilt in guter Näherung $\sin \theta \approx \theta$ und man erhält die Schichtdicke aus:

$$
d=\frac{\lambda}{2 \Delta \theta}
$$

Diese Methode erlaubt allerdings nur die Bestimmung von Schichtdicken im Bereich $10 \mathrm{~nm}<d<150 \mathrm{~nm}$, da die Maxima bei dickeren Schichten zu nah beieinander und bei

\footnotetext{
${ }^{9}$ Finnigan LCQ-Ionenfallen Massenspektrometer (Thermo Finnigan, San Jose, USA).

${ }^{10}$ Daher verwendet man hier auch den Begriff Reflektometrie anstatt Diffraktometrie.

${ }^{11}$ Der Einfluss des Brechungsindex wird hier vernachlässigt, da dieser im Röntgenbereich typischerweise sehr klein ist (Unterschiede $\approx 10^{-5}$ ).
} 
dünneren Schichten zu wenig Maxima im Bereich kleiner Winkel liegen. Hier wird sie vor allem zur Kalibrierung der Schwingquarzwaage in der Aufdampfanlage verwendet.

Nachdem die Lage der Probe mit Hilfe von Rocking-Scans justiert ist, werden die Messungen mit einer Schrittweite von 0,005 und einer Messzeit von $1 \mathrm{~s} /$ step durchgeführt. Die Blenden sind dabei so gewählt, dass der Detektor hinreichend geschützt und dennoch genug Intensität detektierbar ist (Detektor- und Divergenzblende: $0,1 \mathrm{~mm}$, Streustrahlblende 0,2 $\mathrm{mm}$ und Schneidblende: 0,05 mm).

Im Allgemeinen werden Röntgenstrahlen an der Elektronenhülle von Atomen gebeugt und die von den Atomen ausgehenden Wellen interferieren. In Kristallen kommt es durch die periodische Anordnung der Atome nur unter bestimmten Winkeln zu konstruktiver Interferenz - und somit zu Intensitätsmaxima - und zwar genau dann, wenn für die parallelen Netzebenen mit Abstand $d$ die Bragg-Bedingung (s. o.) erfüllt ist. Aus der Lage der Reflexe kann man folglich den Netzebenenabstand bestimmen. Die Breite der Reflexe ist abhängig von der Größe der kohärent streuenden Bereiche und über die Scherrer-Formel lässt sich der Kristallitdurchmesser abschätzen [Sch18]. In amorphen Schichten, die sich durch eine fehlende Fernordnung auszeichnen, erwartet man daher breite Maxima, die durch die Nahordnung nächster Nachbarn bestimmt sind [El190].

Für die Weitwinkelmessungen wird die Probe leicht verkippt eingebaut, um Maxima durch das kristalline Substrat nicht zu detektieren. Die Blenden werden für mehr Intensität deutlich größer gewählt (Detektor- und Streustrahlblende: 0,6 mm, Divergenzblende 0,2 mm und Schneidblende: $\infty$ ). Die Messung erfolgt mit einer Schrittweite von $0,05^{\circ}$ und einer Messzeit von $80 \mathrm{~s} / \mathrm{step}$.

\subsubsection{Größen zur Charakterisierung von Oberflächen}

An dieser Stelle sollen zunächst einige wichtige Größen für die Charakterisierung von Oberflächen eingeführt werden, die es ermöglichen sollen, unterschiedliche Proben zu vergleichen. Neben der visuellen Inspektion von Bildern, die vor allem qualitative Aussagen über die Topographie zulässt, werden mathematische Größen eingeführt, die eine Oberfläche quantitativ beschreiben. Meist beschreibt man eine Oberfläche als orts- und dicken- oder zeitabhängige Höhenfunktion $h(\vec{x}, d, t)$, wobei viele Methoden, wie z. B. die Rasterkraftmikroskopie, keine absoluten Höhenangaben liefern, sondern nur Höhenänderung relativ zur über den Scanbereich gemittelten Höhe $\left(h(\vec{x}, d, t)-\langle h(\vec{x}, d, t)\rangle_{\vec{x}}\right)$. Die zeitliche Entwicklung der Höhe ist dabei, wie auch der Einfluss der Dicke, nicht an einem einzelnen Bild beobachtbar, sondern nur in einer entsprechenden Bilderserie. Daher werden sie für die folgenden Definitionen (vgl. z. B. in [Bar95]) weggelassen.

\section{RMS-Rauigkeit ${ }^{12}$}

$$
\sigma(L)=\sqrt{\left\langle\left(h(\vec{x})-\langle h(\vec{x})\rangle_{\vec{x}}\right)^{2}\right\rangle_{\vec{x}}}
$$

Dabei bezeichnet $L$ die Größe des Bildausschnitts, über den gemittelt wird.

Die RMS-Rauigkeit ist ein quantitatives Maß für die mittlere Abweichung von der durchschnittlichen Höhe.

\footnotetext{
${ }^{12}$ RMS für Root Mean Square.
} 


\section{Höhen-Höhen-Korrelationsfunktion}

$$
C(r)=\left\langle\left(h(\vec{r}+\vec{x})-\langle h(\vec{x})\rangle_{\vec{x}}\right)\left(h(\vec{x})-\langle h(\vec{x})\rangle_{\vec{x}}\right)\right\rangle_{\vec{x},|\vec{r}|=r}
$$

Damit gilt: $C(0)=\left\langle\left(h(\vec{x})-\langle h(\vec{x})\rangle_{\vec{x}}\right)^{2}\right\rangle_{\vec{x}}=\sigma^{2}$.

Die Höhen-Höhen-Korrelationsfunktion beschreibt, wie ähnlich sich Höhenstrukturen im Abstand $r$ sind, beziehungsweise in welchen lateralen Abständen wieder gleiche Höhenstrukturen auftreten. Für kleine $r$ lässt sich der Abfall von $C(r)$ folgendermaßen ausdrücken [Ras82]:

$$
C(r)=\sigma^{2} \exp \left(\frac{-r^{2}}{R_{e}^{2}}\right)
$$

Dabei ist $R_{e}$ die Korrelationslänge, die den mittleren Radius von Hügeln auf der Oberfläche beschreibt. $R_{e}$ entspricht dem Radius, bei dem $C(r)$ auf $\frac{1}{e}$ abgefallen ist.

\section{Höhendifferenzkorrelationsfunktionen der Ordnung $n \in \mathbb{N}$}

$$
H_{n}(r)=\left\langle|h(\vec{r}+\vec{x})-h(\vec{x})|^{n}\right\rangle_{\vec{x},|\vec{r}|=r}
$$

Für $n=2$ spricht man von der Höhendifferenzkorrelationsfunktion (ohne Ordnungsangabe):

$$
\begin{aligned}
H_{2}(r)= & \left\langle|h(\vec{r}+\vec{x})-h(\vec{x})|^{2}\right\rangle_{\vec{x},|\vec{r}|=r} \\
= & \left\langle h(\vec{r}+\vec{x})^{2}-2 h(\vec{r}+\vec{x}) h(\vec{x})+h(\vec{x})^{2}\right\rangle_{\vec{x},|\vec{r}|=r} \\
& \Rightarrow \quad H(r):=H_{2}(r)=2 \sigma^{2}-2 C(r)
\end{aligned}
$$

Das erste Minimum der Höhendifferenzkorrelationsfunktion, welches dem ersten Maximum der Höhen-Höhen-Korrelationsfunktion entspricht, wird als Korrelationslänge $R_{c}$ definiert. $R_{c}$ ist ein Wert für den mittleren Hügelabstand auf der Oberfläche.

\section{Fourierspektrum - Power Spectral Density}

$$
C(q)=\left\langle\left|F T\left\{h(\vec{x})-\langle h(\vec{x})\rangle_{\vec{x}}\right\}\right|^{2}\right\rangle|\vec{q}|=q
$$

Das Fourierspektrum wird auch spektrale Leistungsdichte genannt. Aus dem Maximum des Fourierspektrums erhält man den Korrelationswellenvektor $Q_{c}$, welcher mit der Korrelationslänge über die Beziehung $Q_{c}=\frac{2 \pi}{R_{c}}$ verbunden ist. Des Weiteren kann man aus den Fourierspektren Rückschlüsse auf dominierende Wachstumsprozesse oder Wachstumsinstabilitäten ziehen, da die Intensitäten der verschiedenen Wachstumsmoden angezeigt werden [Her50; Mul57; Mul59; May00]. Dominiert ein Prozess das Wachstum, fällt das Fourierspektrum mit $q^{-i}$ ab, wobei der Exponent $i$ vom Prozess abhängt (z. B. erhält man für viskoses Fließen $i=1$ oder für Oberflächendiffusion $i=4)$.

Außerdem liefert die Rücktransformation

$$
C(r)=F T^{-1}\{C(q)\}
$$

eine schnelle Möglichkeit, die Höhen-Höhen-Korrelationsfunktion zu berechnen [Pre02]. 
(a)

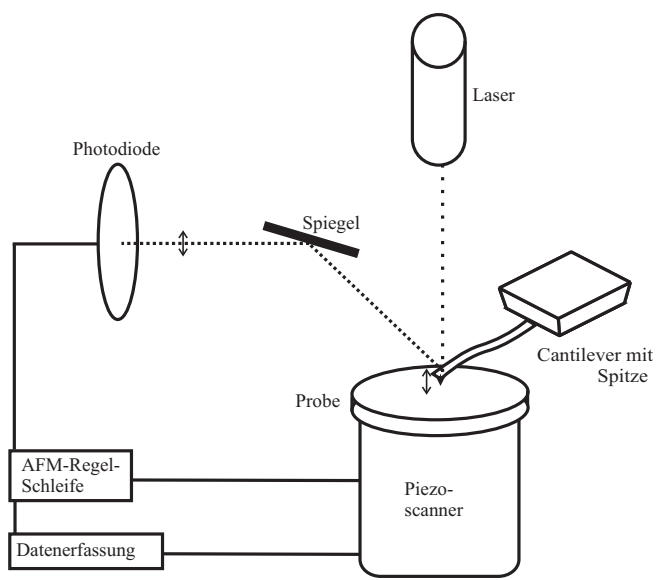

(b)

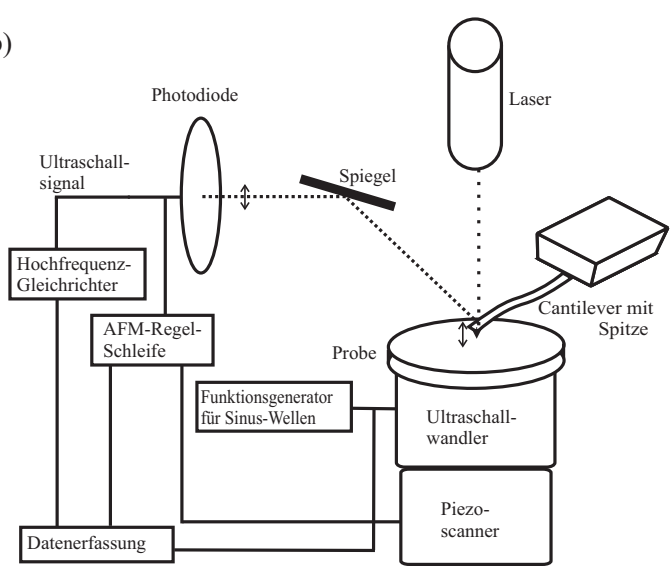

Abbildung 3.3: Schematischer Aufbau (a) eines konventionellen AFMs und (b) eines AFAMs.

\subsubsection{Rasterkraftmikroskopie}

Seit der Erfindung des Rastertunnelmikroskops (STM $\left.{ }^{13}\right) 1981$ von Binnig, Rohrer, Gerber und Weibel [Bin81; Bin82] und des Rasterkraftmikroskops (AFM $\left.{ }^{14}\right) 1986$ von Binnig, Quate und Gerber [Bin86] haben sich die Rastersondenverfahren zur Untersuchung von Oberflächen auf der Nano- bis Mikrometerskala als Standardverfahren etabliert [Cap99; Wei03; Mey04; Seo08; Zav08]. Daher soll an dieser Stelle statt einer ausführlichen Beschreibung nur eine kurze Übersicht gegeben werden, da auch das hier verwendete Gerät bereits ausführlich beschrieben wurde (z. B. in [Vre05]). Für alle AFM Messungen in dieser Arbeit wird ein MultiMode Rastersondenmikroskop mit einem NanoScope IV Controller der Firma Veeco Instruments GmbH verwendet. Bei diesem Gerät wird die Spitzenauslenkung mit einem Laserstrahl, der von der Spitze reflektiert wird, an einer Vier-Segment-Photodiode detektiert. Der maximale Scanbereich beträgt mit dem verwendeten Scanner des Typs AS-12V („, $E^{\prime \prime}$ - vertical) $12 \times 12 \mu \mathrm{m}^{2}$ bei einer Auflösung von typischerweise $512 \times 512$ Datenpunkten $^{15}$. Der Aufbau ist in Abb. 3.3(a) skizziert. Um eine möglichst effiziente Schwingungsdämpfung zu gewährleisten, steht das AFM auf einer schweren Steinplatte, die an Expanderseilen aufgehängt ist. Zur Steuerung des Geräts werden die mitgelieferten Programme NanoScope(R) III, Version 5.30r1 und Version 6.12r1 verwendet, die die meisten in Abs. 3.2.4 beschriebenen Größen ausgeben können. Weitere Auswertungen werden mit IDL ${ }^{16} 6.0$ und MATLAB ${ }^{17}$ R2008a durchgeführt. Je nach Messmethode kommen verschiedene Cantilever zum Einsatz, deren typische Abmessungen in Tab. 3.1 angegeben sind.

\footnotetext{
${ }^{13}$ STM für Scanning Tunneling Microscope.

${ }^{14}$ AFM für Atomic Force Microscope, manchmal auch SFM für Scanning Force Microscope.

${ }^{15}$ Bei Verwendung der Software NanoScope(R) III, Digital Instruments Version 6.12r1 oder höher können auch $4096 \times 1024$ Datenpunkte aufgenommen werden.

${ }^{16}$ IDL für Interactive Data Language.

${ }^{17}$ MATLAB für MATrix LABoratory.
} 


\begin{tabular}{|c|c|c|c|c|c|c|c|}
\hline Тур & $\begin{array}{c}\text { Her- } \\
\text { steller }\end{array}$ & $\begin{array}{c}\text { Mate- } \\
\text { rial }\end{array}$ & $\begin{array}{l}\text { Länge } \\
{[\mu \mathrm{m}]}\end{array}$ & $\begin{array}{r}\text { Breite } \\
{[\mu \mathrm{m}]}\end{array}$ & $\begin{array}{r}\text { Dicke } \\
{[\mu \mathrm{m}]}\end{array}$ & $\begin{array}{c}\text { Feder- } \\
\text { konstante } \\
{[\mathrm{N} / \mathrm{m}]}\end{array}$ & $\begin{array}{c}\text { Resonanz- } \\
\text { frequenz } \\
{[\mathrm{kHz}]}\end{array}$ \\
\hline BS-Tap300 & Budget & $\mathrm{Si}$ & 125 & 30 & 4 & 40 & 300 \\
\hline BS-Multi75 & Budget & $\mathrm{Si}$ & 225 & 28 & 3 & 3 & 75 \\
\hline RTESP & Veeco & $\mathrm{Si}$ & 125 & 35 & 4 & 40 & 300 \\
\hline $\begin{array}{c}\text { NP } \\
(4 \text { Cant.) }\end{array}$ & Veeco & $\mathrm{SiNi}$ & $\begin{array}{l}115 . . \\
. .196\end{array}$ & $\begin{array}{l}17 . . \\
. .41\end{array}$ & 0,6 & $\begin{array}{l}0,06 . . \\
. .0,58\end{array}$ & $\begin{array}{l}18 . . \\
. .57\end{array}$ \\
\hline
\end{tabular}

Tabelle 3.1: Typische Abmessungen der verwendeten Cantilever nach Angaben der Hersteller $($ Budget $=$ Budget Sensors; Veeco $=$ Veeco Instruments GmbH). Als Spitzenradius wird für alle Typen (außer NP) $r \leq 10 \mathrm{~nm}$ angegeben.

\section{Topographiemessungen}

Zur Messung der Topographie wird das AFM im TappingMode ${ }^{T M}$ [Zho93] betrieben, wobei der Regelkreis die Amplitude konstant hält. Dabei kommen Cantilever des Typs BS-Tap300 und BS-Multi75 zum Einsatz (vgl. Tab. 3.1).

\section{Kraft-Abstandskurven}

Eine ausführliche Beschreibung von Kraft-Abstandskurven in AFM Messungen findet man z. B. in [Cap99], daher soll an dieser Stelle nur eine kurze Wiederholung der wichtigsten Fakten erfolgen.

Mit einer Kraft-Abstandskurve beschreibt man die Wechselwirkung zwischen Spitze und Probe in Abhängigkeit vom Abstand. Tatsächlich gemessen wird aber die Auslenkung des Cantilevers $\delta_{c}$ als Funktion der z-Piezo-Bewegung $z$, bzw. des Abstandes zwischen Probenoberfläche und der Ruheposition des Cantilevers. Der wirkliche Abstand $D$ zwischen Spitze und Probe ist gegeben durch:

$$
D=z-\left(\delta_{c}-\delta_{s}\right)
$$

Dabei beschreibt $\delta_{s}$ die Deformation der Probe, die für kleine Kräfte (im elastischen Bereich) vernachlässigt werden kann. Dieser Zusammenhang ist noch einmal in Abb. 3.4(a) dargestellt. Daher gilt der Begriff Kraft-Abstandskurve eigentlich nicht für die vom AFM aufgenommenen Daten, die man besser als Kraft-Verschiebungskurve bezeichnen sollte, sondern nur für die aus den Rohdaten umgerechneten Kurven. Die Kraft zwischen Spitze und Probe ist für kleine Auslenkungen nach dem Hookeschen Gesetz durch die Auslenkung des Cantilevers und dessen Federkonstante $k_{c}$ gegeben: $F=-k_{c} \delta_{c}$. Eine typische Kraft-Verschiebungskurve ist in Abb. 3.4(b) gezeigt. Weit weg von der Oberfläche (Bereiche A und E) ist die Wechselwirkung sehr klein, bzw. null (Nullinie). Nähert sich der Cantilever der Probe, spürt er kurz vor Erreichen der Oberfläche eine attraktive Anziehung (z. B. durch van-der-Waals-Wechselwirkung) und springt in Kontakt mit der Oberfläche (Punkt B). Es folgt die Kontaktlinie oder Kontaktregion (Bereich C). Dieser Bereich beinhaltet Informationen über elastische und plastische Eigenschaften, die man mittels Kontaktmechanik-Modellen berechnen kann. 
(a)

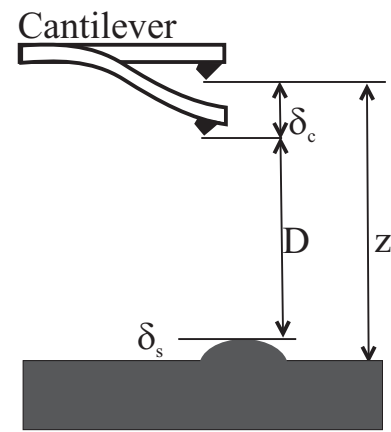

(b)

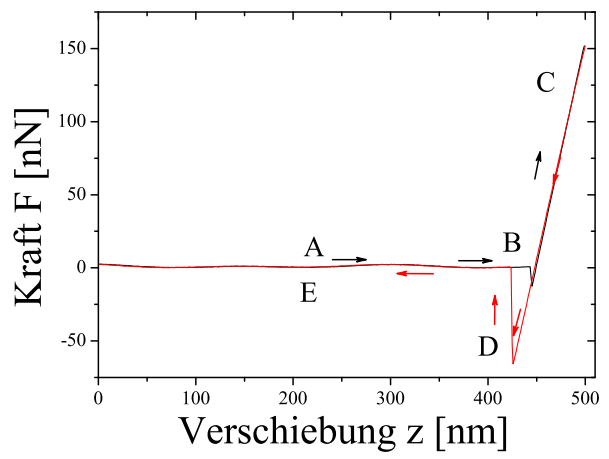

Abbildung 3.4: (a) Zusammenhang von z-Piezo-Bewegung z, Auslenkung des Cantilevers $\delta_{c}$, Deformation der Oberfläche $\delta_{s}$ und tatsächlichem Spitze-ProbeAbstand $D$ (nach [Cap99]). Die Oberflächendeformation $\delta_{s}$ kann dabei sowohl positiv (Erhöhung) als auch negativ (Eindruck) sein. (b) Typische Kraft-Verschiebungskurve gemessen auf einem metallischen Glas.

Beim Zurückfahren bleibt die Spitze durch Adhäsion an der Oberfläche haften, bis sie wieder auf die Nulllinie springt (Punkt D).

Ein einfaches Modell der Kontaktmechanik ist das Hertz-Modell [Her81], das die elastische Verformung von zwei homogenen, glatten Oberflächen unter Last beschreibt. Weder Oberflächenkräfte noch Adhäsion werden in diesem Modell beachtet. Nimmt man die Spitze als Kugel an, erhält man den folgenden Zusammenhang zwischen Kraft $F$ und Deformation $\delta$ :

$$
F=\frac{4}{3} E^{*} \sqrt{R} \delta^{3 / 2}
$$

Dabei bezeichnet $E^{*}$ den reduzierten Elastizitätsmodul (auch red. E-Modul oder red. Young-Modul) und $R$ den Spitzenradius. Der reduzierte E-Modul ergibt sich bei isotropen Materialien aus den elastischen Konstanten (E-Modul $E$ und Poissonzahl $\nu$ ) von Spitze $\left(E_{t}, \nu_{t}\right)$ und Probe $\left(E_{s}, \nu_{s}\right)$ und bei anisotropen Materialien aus den Indentationsmoduli von Spitze $\left(M_{t,\langle h k l\rangle}\right)$ und Probe $\left(M_{s,\langle h k l\rangle}\right)$ :

$$
\begin{aligned}
\frac{1}{E^{*}} & =\frac{1-\nu_{s}^{2}}{E_{s}}+\frac{1-\nu_{t}^{2}}{E_{t}} \quad \mathrm{bzw} . \\
\frac{1}{E^{*}} & =\frac{1}{M_{s,\langle h k l\rangle}}+\frac{1}{M_{t,\langle h k l\rangle}}
\end{aligned}
$$

Eine quantitative Auswertung von Kraft-Abstandskurven ist schwierig, da die Spitzeneigenschaften, vor allem der Spitzenradius nicht genau bekannt sind. Dazu können Fehler in der Cantilever-Auslenkungs-Kalibrierung und Abweichungen von der HertzTheorie kommen. Um dennoch elastische Eigenschaften an verschiedenen Stellen einer Probe miteinander vergleichen zu können, haben E. A. Hassan et. al. das sog. „FIELmapping" vorgeschlagen [AH98]. Dabei werden Kraft-Verschiebungskurven an verschiedenen Positionen im relativen Trigger-Modus ${ }^{18}$ aufgenommen, d. h. die maximale Aus-

\footnotetext{
${ }^{18}$ Messmodus der AFM-Software, bei dem die maximale Auslenkung (Trigger) des Cantilevers vorgegeben wird.
} 
lenkung und damit die maximale Kraft sind an allen Stellen gleich und man vergleicht die Arbeit $W$, die der Cantilever während des Eindrucks leisten muss. Diese ist gegeben durch die Fläche unter der Kraft-Abstandskurve. Für zwei Positionen 1 und 2 gilt dann für eine kugelförmige Spitze (Herleitung in [AH98]):

$$
\frac{W_{1}}{W_{2}}=\left(\frac{E_{1}^{*}}{E_{2}^{*}}\right)^{2 / 3}
$$

Über absolute Werte kann mit dieser Methode keine Aussage getroffen werden, aber räumliche und zeitliche Heterogenitäten lassen sich im Prinzip bestimmen.

Ein großes Problem bei den in dieser Arbeit untersuchten Polymerfilmen ist plastische Verformung durch den Eindruck. Die Theorie beschreibt nur den elastischen Bereich, daher müssen die Kräfte möglichst klein gehalten werden. Zum einen kommen daher Cantilever des Typs BS-Multi75 mit geringerer Federkonstante zum Einsatz. Außerdem wird die maximale Auslenkung so klein wie möglich gewählt, allerdings ist die Auswertung bei Auslenkungen kleiner als $5 \mathrm{~nm}$ kaum möglich.

Um Elastizitätskarten zu erstellen, werden an bis zu $126 \times 124$ Stellen (oft nur $65 \times 63$, um die Messzeit zu verkürzen und somit den Einfluss von Drift zu verringern) mit Abständen von $10-20 \mathrm{~nm}$ Kraft-Verschiebungskurven mit einer Rate von $1-2 \mathrm{~Hz}$ aufgenommen. Die Kurven werden mit IDL in Kraft-Abstandskurven umgerechnet und die Fläche unter der Annäherungskurve bestimmt. Diese Messungen sind sehr zeitintensiv (ca. 15 h für eine vollständige Karte), wodurch Drift und Temperaturschwankungen einen großen Einfluss haben. Daher wird jede Scan-Linie mit ihrem Mittelwert normiert.

\section{Ultraschall-Kraftmikroskopie}

Eine andere Möglichkeit, lokale elastische Eigenschaften zu messen, bietet die Ultraschall-Kraftmikroskopie, die am Fraunhofer Institut für zerstörungsfreie Prüfverfahren (IZFP) Saarbrücken von U. Rabe und W. Arnold entwickelt wurde [Rab94a; Rab94b; Rab96a; Rab96b]. Dabei nutzt man aus, dass sich das Schwingungsspektrum eines Cantilevers gegenüber seiner freien Schwingung ändert, wenn die Spitze in Kontakt mit einer Probe kommt. Die Steifigkeit dieses Kontaktes $k^{*}$, definiert durch die Steigung der Kraftkurve am Arbeitspunkt, hängt maßgeblich von den elastischen Konstanten von Probe und Spitze und von der Geometrie des Kontaktes ab. Eine ausführliche Herleitung und Diskussion der folgenden Ausführungen findet sich in [KM05; Rab06; KM08].

Der Cantilever kann als elastischer Balken der Länge $L$ mit konstantem Querschnitt angenommen werden, der an einer Seite fest eingespannt ist. Dieser kann in verschiedenen Schwingungsmoden (Biegemoden, longitudinale Stabmoden, Torsionsmoden) mit unterschiedlichen Frequenzen schwingen. Die erste Biegemode wird z. B. im TappingMode $^{T M}$ eingesetzt. Für die Ultraschall-Kraftmikroskopie werden in erster Linie die Biegemoden verwendet, die z. B. in [Rab06] dargestellt sind. Ist das andere Ende des Cantilevers frei, gilt für gedämpfte Biegeschwingungen die folgende Differentialgleichung [Rab06]:

$$
E I \frac{\partial^{4} y(x, t)}{\partial x^{4}}+\eta_{L} \rho A \frac{\partial y(x, t)}{\partial t}+\rho A \frac{\partial^{2} y(x, t)}{\partial t^{2}}=0
$$


(a)

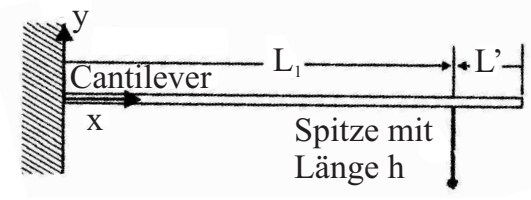

(b)

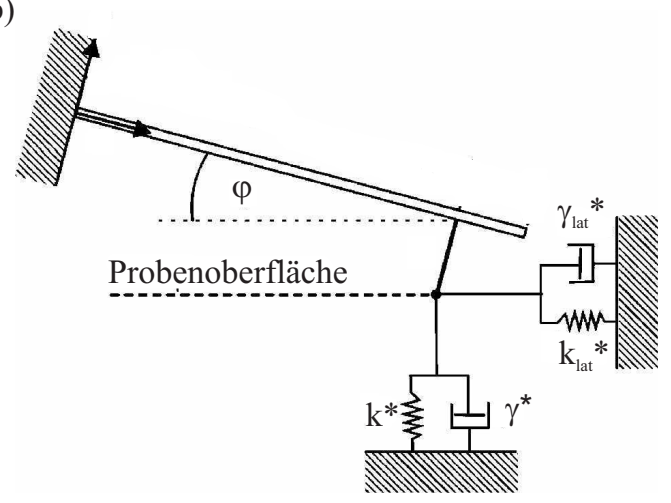

Abbildung 3.5: Mechanisches Modell eines AFM Cantilevers als elastischer Balken der Länge $L=L_{1}+L^{\prime}$, ohne (a) und mit Kontakt (b) zu einer Probe (nach [KM08]).

Dabei ist $y(x, t)$ die Auslenkung des Cantilevers am Punkt $x$ (vgl. Abb. 3.5), $E$ der E-Modul in Längsrichtung, $I=w b^{3} / 12$ mit Balkenbreite $w$ und Balkendicke $b$ das Flächenträgheitsmoment, $\eta_{L}$ eine Dämpfungskonstante gegeben durch die umgebende Luft, $\rho$ die Dichte und $A$ die Querschnittsfläche.

Mit den Randbedingungen, dass der Cantilever bei $x=0$ fest eingespannt und bei $x=$ $L$ kräftefrei sein muss ${ }^{19}$, erhält man eine charakteristische Gleichung, die die diskreten Lösungen von Gl. (3.13) definiert. Diese lässt sich numerisch lösen [Rab06; KM08]. Für die n-te Mode des Balkens erhält man die folgenden Lösungen (Frequenzen) mit den dimensionslosen Wellenzahlen $\alpha_{n} L$ :

$$
f_{n}=\frac{\left(\alpha_{n} L\right)^{2}}{c_{c}^{2}} \quad \text { mit } \quad c_{c}=L \sqrt{2 \pi \sqrt{\frac{\rho A}{E I}}}
$$

Die ersten sieben Biegemoden sind in [Rab06] numerisch berechnet und aufgelistet, so gilt z. B. für die ersten beiden freien Biegemoden: $\left(\alpha_{1} L\right)=1,875$ und $\left(\alpha_{2} L\right)=4,694$. Biegemoden sind dispersiv und der Abstand zwischen zwei Moden nimmt mit steigender Frequenz zu. Es gilt die Dispersionsrelation:

$$
\frac{f}{f_{1, \text { frei }}}=\frac{(\alpha L)^{2}}{\left(\alpha_{1, \text { frei }} L\right)^{2}}
$$

Im Kontakt mit einer Probe ändern sich die Randbedingungen. Für genügend hohe Auflagekräfte (Kontaktregion in der Kraft-Abstandskurve, so dass Adhäsionskräfte vernachlässigt werden können) kann man die auftretenden Kräfte durch Federn ( $k^{*}$ und $\left.k_{\text {lat }}^{*}\right)$ und Dämpfungstöpfe $\left(\gamma\right.$ und $\left.\gamma_{l a t}\right)$ nähern, wie es in Abb. 3.5(b) schematisch dargestellt ist. Dadurch werden die Resonanzfrequenzen verschoben und aus der Verschiebungsweite kann man die Kontaktsteifigkeit bestimmen - die vertikale Kontaktsteifigkeit ist durch $k^{*}=-\partial F(z) /\left.\partial z\right|_{z=z_{\text {Arbeitspunkt }}}$ definiert. Da die Dämpfung aufgrund der hohen Güte der Cantilever im Allgemeinen klein ist und die Resonanzkurven auch im Kontakt scharf bleiben, werden die Dämpfungstöpfe im Folgenden, wie allgemein

\footnotetext{
${ }^{19}$ Insbesondere müssen das Drehmoment $E I \frac{\partial^{2} y(x, t)}{\partial x^{2}}$ und die Scherkraft $E I \frac{\partial^{3} y(x, t)}{\partial x^{3}}$ verschwinden.
} 
üblich, vernachlässigt. Unter diesen Voraussetzungen ändert sich die charakteristische Gleichung zu:

$$
\begin{array}{r}
A_{0} c_{P}\left(\frac{h}{L_{1}}\right)^{2}\left(3 \frac{k^{*}}{k_{c}}\right)^{2}+3 A_{1} \frac{k^{*}}{k_{c}}\left(\cos ^{2} \varphi+c_{P} \sin ^{2} \varphi\right)+3 A_{2} \frac{h}{L_{1}} \frac{k^{*}}{k_{c}} \\
\times \sin \varphi \cos \varphi\left(c_{P}-1\right)+3 A_{3}\left(\frac{h}{L_{1}}\right)^{2} \frac{k^{*}}{k_{c}}\left(\sin ^{2} \varphi+c_{P} \cos ^{2} \varphi\right)+A_{4}=0
\end{array}
$$

Der Faktor $c_{P}=\frac{k_{l a t}^{*}}{k^{*}}$ beschreibt das Verhältnis zwischen lateraler und vertikaler Kontaktsteifigkeit und wird typischerweise mit $c_{P}=0,85$ angenommen. Wie in Abb. 3.5(a) abgebildet bezeichnen $L_{1}$ den Abstand von Einklemmung bis zur Spitze, $L^{\prime}$ den Abstand von Spitze zum Ende des Cantilevers, $L=L_{1}+L^{\prime}$ die Gesamtlänge des Cantilevers, $h$ die Spitzenhöhe und $\varphi$ den (technisch bedingten) Neigungswinkel des Cantilevers gegen die Probenoberfläche. Die restlichen Koeffizienten $A_{0}$ bis $A_{4}$ sind folgendermaßen definiert:

$$
\begin{aligned}
A_{0}= & \left(1-\cos \alpha L_{1} \cosh \alpha L_{1}\right)\left(1+\cos \alpha L^{\prime} \cosh \alpha L^{\prime}\right) \\
A_{1}= & \alpha L_{1}\left[-\left(1-\cos \alpha L_{1} \cosh \alpha L_{1}\right)\left(\sin \alpha L^{\prime} \cosh \alpha L^{\prime}-\sinh \alpha L^{\prime} \cos \alpha L^{\prime}\right)\right. \\
& \left.+\left(1+\cos \alpha L^{\prime} \cosh \alpha L^{\prime}\right)\left(\sin \alpha L_{1} \cosh \alpha L_{1}-\sinh \alpha L_{1} \cos \alpha L_{1}\right)\right] \\
A_{2}= & 2\left(\alpha L_{1}\right)^{2}\left[\sin \alpha L_{1} \sinh \alpha L_{1}\left(1+\cos \alpha L^{\prime} \cosh \alpha L^{\prime}\right)\right. \\
& \left.+\sin \alpha L^{\prime} \sinh \alpha_{n} L^{\prime}\left(1-\cos \alpha L_{1} \cosh \alpha L_{1}\right)\right] \\
A_{3}= & \left(\alpha L_{1}\right)^{3}\left[\left(\sin \alpha L_{1} \cosh \alpha L_{1}+\sinh \alpha L_{1} \cos \alpha L_{1}\right)\left(1+\cos \alpha L^{\prime} \cosh \alpha L^{\prime}\right)\right. \\
& \left.-\left(\sin \alpha L^{\prime} \cosh \alpha L^{\prime}+\sinh \alpha L^{\prime} \cos \alpha L^{\prime}\right)\left(1-\cos \alpha L_{1} \cosh \alpha L_{1}\right)\right] \\
A_{4}= & 2\left(\alpha L_{1}\right)^{4}(1+\cos \alpha L \cosh \alpha L)
\end{aligned}
$$

Gl. (3.16) ist eine quadratische Gleichung für die Kontaktsteifigkeit $k^{*}$. Als Lösung findet man:

$$
\begin{aligned}
k^{*} & =\frac{k_{c}}{3}\left(z \pm \sqrt{z^{2}-\left(\frac{L_{1}}{h}\right)^{2} \frac{A_{4}}{c_{P} A_{0}}}\right) \quad \text { mit } \\
z & =-\frac{A_{1}\left(\cos ^{2} \varphi+c_{P} \sin ^{2} \varphi\right)+A_{2} \frac{h}{L_{1}}\left(c_{P}-1\right) \sin \varphi \cos \varphi+A_{3}\left(\frac{h}{L_{1}}\right)^{2}\left(\sin ^{2} \varphi+c_{P} \cos ^{2} \varphi\right)}{2 A_{0} c_{P}\left(\frac{h}{L_{1}}\right)^{2}}
\end{aligned}
$$

Aus den Messungen der Kontaktresonanzen $f_{\text {kont }}$ und den freien Resonanzen $f_{\text {frei }}$ kann man über die Dispersionsgleichung (3.15), die für alle Biegemoden unabhängig von den Randbedingungen gilt, die fehlenden Wellenzahlen $\alpha L$ berechnen:

$$
\alpha L=(\alpha L)_{\text {frei }} \sqrt{\frac{f_{\text {kont }}}{f_{\text {frei }}}}
$$


Somit ist im Prinzip eine quantitative Messung der Kontaktsteifigkeit $k^{*}$ möglich, wenn man die Eigenschaften des Cantilevers - wie die Längen $L, L_{1}, L^{\prime}$, die Spitzenhöhe, den Neigungswinkel und die Federkonstante - genau kennt. Alle Angaben werden vom Hersteller angegeben, allerdings sind die Schwankungen teilweise sehr groß. Die Längen lassen sich mit einem Lichtmikroskop messen, für die Spitzenhöhe benötigt man dagegen ein Rasterelektronenmikroskop. Die Bestimmung der Federkonstante ist immer noch ein aktuelles Forschungsgebiet [Gat07]. Da auch die Cantilever- und Spitzenform nicht exakt den Annahmen entsprechen, können die absoluten Werte von den tatsächlichen (stark) abweichen. Durch den Vergleich zur freien Resonanz und die Kalibrierung der Spitze (s. u.) mit mindestens zwei Referenzproben werden diese Ungenauigkeiten minimiert. Wichtiger als die absoluten Zahlen sind für diese Arbeit die lokalen Abweichungen auf einer Probe, die mit dieser Methode sehr gut gemessen werden können, solange man im elastischen Kraftbereich misst und die Probe nicht plastisch verformt.

Ist die Kontaktsteifigkeit aus den Kontaktresonanzen bestimmt, kann man aus dieser elastische Eigenschaften extrahieren. Unter der Annahme eines Hertzschen Kontakts und einer kugelförmiger Spitze gilt nach Gl. (3.10):

$$
k^{*}=-\frac{\partial F}{\partial z}=\sqrt[3]{6 F R E^{* 2}}
$$

Durch Messungen der Kontaktresonanzen auf zwei Referenzsubstraten (Indizes 1 und 2) mit bekannten Indentationsmoduli kann der Indentationsmodul der Spitze bestimmt werden:

$$
M_{t}=\frac{M_{1} M_{2}\left(1-\sqrt{k_{1}^{* 3} / k_{2}^{* 3}}\right)}{M_{2} \sqrt{k_{1}^{* 3} / k_{2}^{* 3}}-M_{1}}
$$

Außerdem kann man durch vergleichende Messungen bei gleicher Auflagekraft den reduzierten E-Modul einer Probe bestimmen:

$$
E^{*}=E_{\text {Ref }}^{*}\left(\frac{k^{*}}{k_{\text {Ref }}^{*}}\right)^{3 / 2}
$$

Um den Kontakt anzuregen, stehen zwei verschiedene Möglichkeiten zur Auswahl. Bei der als $\mathrm{UAFM}^{20}$ bekannten Methode von Yamanaka und Nakano [Yam96] wird der Cantilever über die Piezoelemente des Halters angeregt, während bei der auch hier verwendeten AFAM Technik ein Ultraschall-Prüfkopf unter der Probe den Kontakt anregt.

Die Ultraschall-Mikroskopie lässt sich in zwei verschiedenen Modi verwenden, dem qualitativen Bild-Modus und dem quantitativen Spektroskopie-Modus. Im Bild-Modus wird im Kontaktmodus des AFMs ein Bild gescannt, während der Kontakt mit einer festen Frequenz in der Nähe einer Kontaktresonanzfrequenz angeregt wird. Neben der Topographie misst man die Amplitude mit der der Cantilever schwingt. Die Amplitude ändert sich, wenn sich die Steifigkeit - und damit die elastischen Eigenschaften der Probe, da alle anderen Werte idealerweise unverändert bleiben - ändert. Je nachdem auf welcher Flanke (absteigend oder aufsteigend) der Resonanzkurve gemessen wird, ist die

\footnotetext{
${ }^{20}$ UAFM für Ultrasonic Atomic Force Microscope.
} 

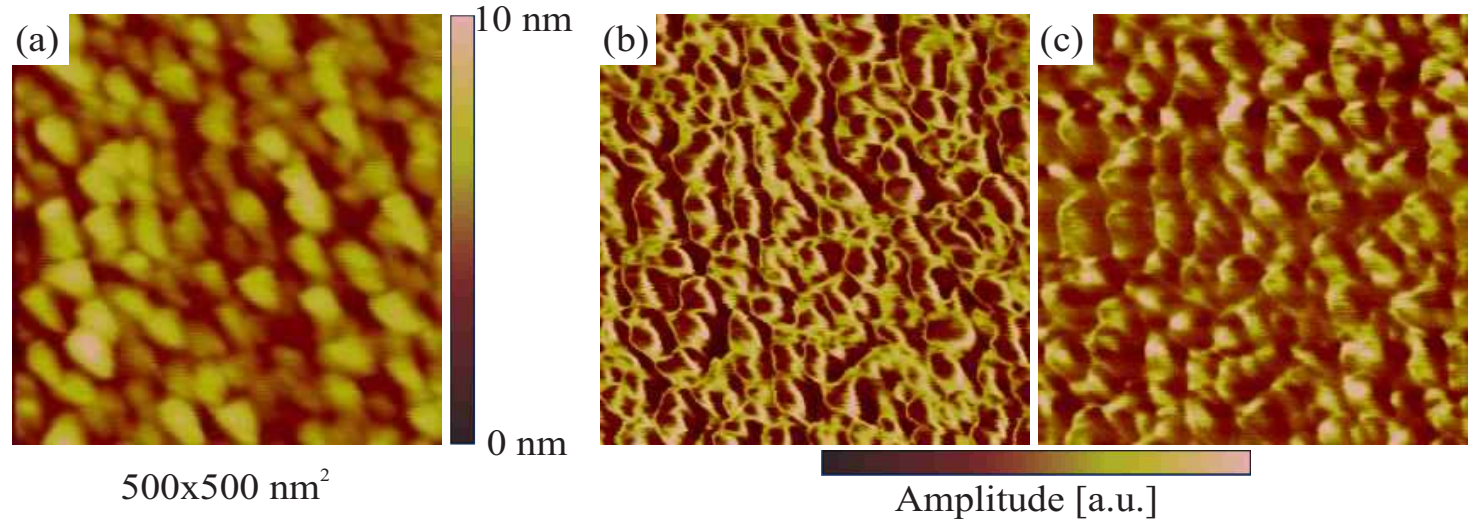

Abbildung 3.6: (a) Topographie einer Nickel Schicht gemessen im Kontaktmodus zusammen mit AFAM Messungen bei verschiedenen Anregungsfrequenzen: (b) $2550 \mathrm{kHz}$ und (c) $2580 \mathrm{kHz}$. Die Resonanzfrequenz liegt bei 2555 kHz. Man erkennt die Kontrastumkehr von Bild (b) zu (c).

Amplitude bei härteren Bereichen höher (linke Flanke) oder niedriger (rechte Flanke). Durch Verschieben der Anregungsfrequenz kann man somit eine Kontrastumkehr im Bild erhalten, wie es in Abb. 3.6 für eine Nickel-Probe gezeigt ist.

Für eine quantitative Analyse muss an jedem Punkt eines Bildes eine Resonanzkurve gemessen und daraus die Kontaktresonanzfrequenz bestimmt werden. Daraus können dann Karten für die Kontaktsteifigkeit, bzw. den Indentationsmodul erstellt werden. Es bleibt anzumerken, dass sich die Kontaktsteifigkeit bei einer kugelförmigen Spitze mit der statischen Auflagekraft erhöht, da sich die Kontaktfläche vergrößert. Bei einer zylinderförmigen Spitze ist dies nicht der Fall.

Im Rahmen dieser Arbeit ist für das MultiMode Rastersondenmikroskop eine AFAMErweiterung aufgebaut und in Zusammenarbeit mit H. Wagner (aus der Arbeitsgruppe von Prof. K. Samwer am I. Physikalischen Institut, Universität Göttingen) in Betrieb genommen worden, wie sie schematisch in Abb. 3.3(b) dargestellt ist. Zwischen Probe und Piezoscanner des AFMs wird ein Ultraschall-Prüfkopf des Typs V133-SM ${ }^{21}$ mit 2, $25 \mathrm{MHz}$ Mittenfrequenz eingesetzt, der longitudinale Schallwellen aussendet. Die Probe wird mit einer dünnen Honigschicht angekoppelt. Dieser Aufbau ist in Abb. 3.7 zu sehen. Ein in einem externen Computer integrierter mit LabVIEW ${ }^{22}$ gesteuerter Funktionsgenerator NI PCI-5402 ${ }^{23}$, der Sinusschwingungen bis $20 \mathrm{MHz}$ erzeugen kann, steuert den Prüfkopf an. Der Funktionsgenerator kann sowohl Dauerstrich-Signale ausgeben, als auch im Sweep-Modus betrieben werden, also einen vorgegebenen Frequenzbereich in kurzer Zeit durchlaufen (min. $1 \mathrm{~ms}$ pro Frequenzschritt). Die Schwingung des Cantilevers wird mit der Photodiode des AFM Messkopfs, die Frequenzen bis zu $5 \mathrm{MHz}$ aufösen kann, detektiert und das Signal direkt hinter dem Messkopf gesplittet. Während ein Teil für die eigentliche AFM-Regelung und Messung verwendet wird, wird ein anderer Teil abgegriffen, um das Ultraschall-Signal auszuwerten. Ein Hochfrequenz (HF)-Gleichrichter liefert die Amplitude des HF-Signals. Diese kann einerseits

\footnotetext{
${ }^{21}$ Dabei handelt es sich um einen Videoscan Breitbandprüfkopf mit 6,3 mm Durchmesser.

${ }^{22}$ Version LabVIEW 7.1 der Firma National Instruments.

${ }^{23}$ PCI Karte der Firma National Instruments.
} 

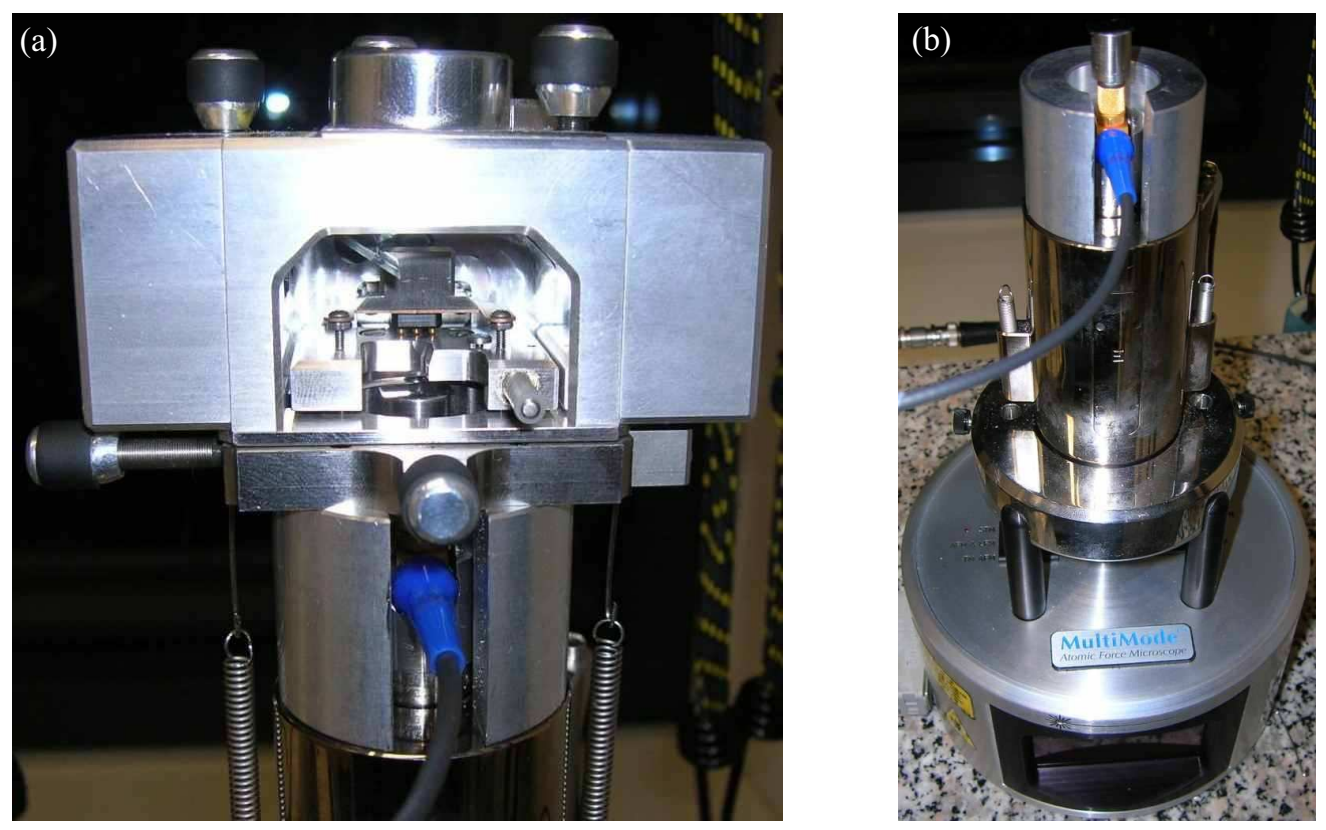

Abbildung 3.7: Erweiterung des MultiMode AFMs zu einem AFAM. Zu sehen ist der AFM Messkopf mit Ultraschall-Prüfkopf und Probe (a) und das MultiMode mit Ultraschall-Prüfkopf aber ohne den AFM Messkopf (b).

als externes Signal in den NanoScope Controller eingekoppelt werden, um ein AFAMBild aufzunehmen. Andererseits kann es auch in dem externen Computer mit Hilfe des Multifunktions-Datenerfassungsmoduls NI PCI-6040E in LabVIEW eingelesen werden, um eine Resonanzkurve aufzunehmen. Die Auflagekraft wird über die AFM Software eingestellt - ebenso die Scan-Parameter für das AFAM-Bild. Der AFM Messrechner und der externe Computer zur Frequenzmessung sind über die „EOL“24 und „EOF“25 Ausgabe des NanoScope Controllers synchronisiert, die einen Puls am Ende einer Zeile bzw. eines Bildes ausgeben. Das LabVIEW-Programm nutzt diese Ausgabe, um zur richtigen Zeit mit den Frequenz-Sweeps zu beginnen. Um eine Elastizitätskarte aufzunehmen, wird die Auto Scan-Funktion der AFM Software genutzt. Die Bildgröße wird auf $\approx 0 \mathrm{~nm}$ gesetzt, die Spitze steht also praktisch still und es werden Bilder an Positionen mit vorgegebenem Abstand untereinander aufgenommen und gleichzeitig die Resonanzkurven gemessen. Für eine Resonanzkurve wird die Frequenz typischerweise über 500 Punkte durch einen vorgegebenen Frequenzbereich gefahren. Bei einer Messzeit von $1 \mathrm{~ms}$ pro Punkt beträgt die Sweepdauer 0,5 s. Für eine Resonanzkurve wird über 510 Sweeps gemittelt. Als Referenzsubstrate werden $\mathrm{SiO}_{2}\left(M_{i s o} \approx 76 \mathrm{GPa}\right)$, Saphir(101) $\left(M_{(101)} \approx 423 \mathrm{GPa}\right)$ und Strontiumtitanat $(100)\left(M_{(100)} \approx 299 \mathrm{GPa}\right)$ verwendet. Aus den Resonanzkurven wird anschließend in MATLAB durch Anpassung einer Lorentz-Kurve das Maximum bestimmt, die Kontaktsteifigkeit und der Indentationsmodul berechnet und eine entsprechende Karte erstellt. ${ }^{26}$

\footnotetext{
${ }^{24} \mathrm{EOL}$ für End Of Line.

${ }^{25}$ EOF für End Of Frame.

${ }^{26}$ Die Implementierung und Weiterentwicklung der Auswerteroutinen in MATLAB werden von H. Wagner vorgenommen.
} 


\section{Fehlerquellen bei AFM Messungen}

Eine der größten Fehlerquellen aller Rastersondentechniken ist die Spitzenqualität. Eine stumpfe, abgeflachte oder breite Spitze ist nicht in der Lage, kleine Strukturen richtig aufzulösen. Stattdessen wird bei kleinen, spitzen Strukturen die Spitzenform abgebildet oder bei schmalen Gräben dringt die Spitze nicht bis zum Boden vor, wodurch die Strukturen flacher erscheinen können.

Störende Einflüsse durch ungewollte Schwingungen kann man durch die Aufhängung des Gerätes minimieren (s. o.). Akustische Störungen, wie z. B. durch laute Unterhaltungen, werden so nicht verhindert, dafür bleibt nur, am Messplatz möglichst Lärm zu vermeiden.

Bei mechanischen Messungen geht oft die Kontaktfläche ein, die bei einer stumpfen Spitze deutlich größer als bei einer scharfen ist. Um Fehler durch die Spitze zu minimieren, misst man Proben z. B. mit unterschiedlichen Spitzen. Bei mechanischen Messungen kalibriert man die Spitze vor und nach den Messungen an einer bekannten Referenz. Außerdem wird nicht direkt die Verbiegung des Cantilevers, sondern das Photodiodenreferenzsignal gemessen, dass für jeden Cantilever kalibriert werden muss und so eine weitere Fehlerquelle darstellt.

Da diese systematischen Fehler sehr komplex und schwer abzuschätzen sind, beinhalten die meisten Fehlerangaben vor allem in Abs. 6 statistische Fehler aus Mittelwertbildung oder Fit-Fehler. 



\section{Kontinuumsmodellierung}

In diesem Kapitel sollen zunächst die wesentlichen Grundlagen für die Modellierung von Schichtwachstum mit Kontinuumsgleichungen vorgestellt werden. Diese erlauben eine mesoskopische Beschreibung von Oberflächenstrukturen, im Gegensatz zur atomistischen Modellierung mit Molekulardynamik- oder Monte Carlo Simulationen. Anschließend wird ein Minimalmodell präsentiert, mit dem das Wachstum von dünnen aufgedampften PC Schichten simuliert werden soll. Nach einer Stabilitätsanalyse folgen am Ende des Kapitels Hinweise zur Implementierung sowie zur Wahl der Parameter.

\subsection{Grundlagen der Kontinuumsmodellierung}

Wie bereits in Abs. 3.2.4 erwähnt, kann eine Oberfläche beschrieben werden, indem man jedem Ort $\vec{x}=(x, y)$ in einem 2-dimensionalen Koordinatensystem, das in der Substratebene liegt, zu jeder Zeit $t$ eine Höhe zuweist $^{1}: h=h(\vec{x}, t)$ [Edw82; Bar95]. Zur Modellierung von Wachstumsprozessen ist die zeitliche Entwicklung dieser Höhenfunktion entscheidend. Ausgehend von einer anfangs glatten Oberfläche, lässt sich diese durch partielle stochastische Differentialgleichungen des Langevin-Typs [Sch97] beschreiben, da Aufdampf- und Diffusionsprozesse stochastischer Natur sind. Ein üblicher Ansatz lautet [Bar95]:

$$
\frac{\partial h(\vec{x}, t)}{\partial t}=F[h(\vec{x}, t)]+I+\eta(\vec{x}, t)
$$

Dabei beschreibt $I$ den mittleren Fluss ankommender Teilchen, also die Depositionsrate, die als räumlich und zeitlich konstant angenommen wird. Das Funktional $F[h(\vec{x}, t)]$ beinhaltet alle Relaxationsprozesse und $\eta$ bezeichnet ein weißes Rauschen, d. h. ein räumlich und zeitlich unkorreliertes stochastisches Rauschen, mit Mittelwert null. Folglich gilt:

$$
\begin{aligned}
\langle\eta(\vec{x}, t)\rangle_{E} & =0 \\
\left\langle\eta(\vec{x}, t) \eta\left(\vec{x}^{\prime}, t^{\prime}\right)\right\rangle_{E} & =2 R \delta\left(\vec{x}-\vec{x}^{\prime}\right) \delta\left(t-t^{\prime}\right)
\end{aligned}
$$

Hier gibt $R=\frac{1}{2} I \Omega$ mit dem Teilchenvolumen $\Omega$ die Rauschstärke an. $\delta$ ist die DeltaDistribution und mit \langle\rangle$_{E}=\langle\rangle_{\text {Ensemble }}$ wird die Mittelung über das gesamte Ensemble bezeichnet. Ein etwas anderer Ansatz, der auch in dieser Arbeit gewählt ist, kombiniert die Depositionsrate und das Rauschen zu einem effektiven Rauschterm $\xi(\vec{x}, t)$ [Edw82; Ton94] mit denselben Eigenschaften wie $\eta(\vec{x}, t)$ (Gl. (4.3)), aber mit $I=\langle\xi(\vec{x}, t)\rangle_{E}$. Gl. (4.1) ändert sich somit zu:

$$
\frac{\partial h(\vec{x}, t)}{\partial t}=F[h(\vec{x}, t)]+\xi(\vec{x}, t)
$$

\footnotetext{
${ }^{1}$ Diese Art der Parametrisierung wird Monge-Parametrisierung genannt.
} 


\subsection{Modell zum Schichtwachstum dünner Polymerfilme}

\subsubsection{Entwicklung der Kontinuumsgleichung}

Im Folgenden soll ein minimales Modell vorgestellt werden, das nur die wesentlichen Prozesse, die beim Wachstum dünner Polymerfilme auftreten, einschließt.

Änderungen im Höhenprofil einer Oberfläche beinhalten immer Materialtransport in ein Gebiet hinein oder aus diesem heraus, also einen Teilchenstrom $\vec{j}$. Für diffusive Prozesse ist ein Gradient im chemischen Potential die Triebkraft für einen Teilchenstrom, $\vec{j} \propto-\nabla \mu$. Der Teilchenstrom ist über die Kontinuitätsgleichung mit dem Höhenprofil verbunden:

$$
\frac{\partial h}{\partial t}=-\nabla \vec{j} \propto \nabla^{2} \mu
$$

Hier sollen zwei Beiträge zum chemischen Potential betrachtet werden: Die Oberflächenspannung $\gamma$ und das effektive Grenzflächenpotential $\Phi$ [Mul57; Vri66; Isr92; Sha93]:

$$
\mu=\Omega\left(-\gamma \nabla^{2} h+\left.\Phi^{\prime}(h)\right|_{\vec{x}}\right)
$$

Dabei bezeichnet $\left.\Phi^{\prime}(h)\right|_{\vec{x}}$ die 1. Ableitung des effektiven Grenzflächenpotentials nach $h$, ausgewertet an der Stelle $\vec{x}$. Mit der Form $\Phi(h)=\frac{\epsilon}{(h+d)^{8}}-\frac{A}{12 \pi(h+d)^{2}}$ kann das effektive Grenzflächenpotential beschrieben werden [Isr92], wie es z. B. auch bei der Beschreibung von Entnetzungsphänomenen verwendet wird [See01a; Bec03]. Diese Form beinhaltet kurzreichweitige repulsive Kräfte, deren Stärke durch den Parameter $\epsilon$ gegeben ist, sowie langreichweitige van-der-Waals Wechselwirkungen, gegeben durch die effektive Hamaker-Konstante $A$. Abweichend von der üblichen Definition des effektiven Grenzflächenpotentials wird in dieser Arbeit ein kleiner Parameter $d$, entsprechend der Ausdehnung der deponierten Teilchen, eingeführt, der das Auftreten von Singularitäten bei sehr kleinen Bedeckungen des Substrats verhindern soll ${ }^{2}$.

Aufgrund der Oberflächenspannung ändert sich das chemische Potential mit der lokalen Krümmung der Oberfläche, die z. B. durch Rauigkeiten entsteht [Mul57]. Unter der Annahme, dass Diffusion, getrieben durch Gradienten im chemischen Potential, der dominierende Relaxationsprozess ist, erhält man aus Gl. (4.5) mit einem Diffusionskoeffizienten $D$ :

$$
F[h(\vec{x}, t)]=D \nabla^{2} \mu
$$

Über $D=D_{S} \Omega \nu /\left(k_{B} T\right)$ ist $D$ proportional zum Oberflächendiffusionskoeffizienten $D_{S}[$ Mul57; Mul59]. Dabei bezeichnen $\nu$ die Anzahl der Teilchen pro Einheitsfläche an der Oberfläche, $k_{B}$ die Boltzmann-Konstante und $T$ die Temperatur.

Die vollständige Gleichung, die es zu lösen gilt, lautet somit:

$$
\frac{\partial h}{\partial t}=D \Omega \nabla^{2}\left(-\gamma \nabla^{2} h-\frac{8 \epsilon}{(h+d)^{9}}+\frac{A}{6 \pi(h+d)^{3}}\right)+\xi(\vec{x}, t)
$$

\footnotetext{
${ }^{2}$ Für $h \rightarrow 0$ divergiert $\Phi$. Große Steigungen können numerisch nur schwer behandelt werden und werden durch den Parameter $d$ umgangen.
} 


\subsubsection{Stabilitätsanalyse der Kontinuumsgleichung}

Gl. (4.8) lässt sich nicht analytisch lösen. Um dennoch zu untersuchen, ob und in welchen Fällen Strukturbildung auf einer anfangs ebenen Oberfläche überhaupt stattfinden kann, hilft eine lineare Stabilitätsanalyse. Daraus gewinnt man die am schnellsten anwachsende Mode, die dann die Strukturbildung dominiert. Gibt es keine ausgezeichnete Mode, wächst die Struktur gleichmäßig und die Oberfläche bleibt stabil. Um die linearisierte Version von Gl. (4.8) zu erhalten, wird $\Phi^{\prime}(h)$ um die mittlere Schichtdicke $h_{0}$ entwickelt. Für diese linearisierte Form existiert eine analytische Lösung für die Fouriertransformierte bezüglich der Ortskoordinate $\hat{h}(\vec{q}, t)=F T[h(\vec{x}, t)]$ [Vil91; May01]:

$$
\left\langle|\hat{h}(\vec{q}, t)|^{2}\right\rangle_{|\vec{q}|=q}=R \frac{1-\exp \left(-2 t D \Omega\left(\Phi^{\prime \prime}\left(h_{0}\right) q^{2}+\gamma q^{4}\right)\right)}{D \Omega\left(\Phi^{\prime \prime}\left(h_{0}\right) q^{2}+\gamma q^{4}\right)}
$$

Damit der Ausdruck auf der rechten Seite ein Maximum aufweist und somit eine Mode stärker wächst und Strukturbildung möglich ist, muss $\Phi^{\prime \prime}\left(h_{0}\right)<0$ gelten, ansonsten steigt der Ausdruck monoton an und es kann kein Maximum geben. Damit $\Phi^{\prime \prime}\left(h_{0}\right)<0$ gelten kann, muss die Hamaker-Konstante positiv sein. Somit führt $A<0$ zu stabilen Oberflächen und $A>0$ zur Instabilität. Aus diesem Grund werden im Folgenden auch die Bezeichnungen ,stabil" für $A<0$ und ,instabil" für $A>0$ verwendet. Für $\Phi^{\prime \prime}\left(h_{0}\right)<0$ gibt es ein Maximum bei $q_{m}=\sqrt{-\Phi^{\prime \prime}\left(h_{0}\right) /(2 \gamma)}$ und daraus folgt Strukturbildung mit einer Längenskala $2 \pi / q_{m}$. Diese Analyse ist allerdings nur zu Beginn des Wachstumsstadiums erlaubt, da sich $h_{0}$ mit der Zeit ändert und die Oberfläche nicht unbedingt glatt bleibt.

\subsubsection{Numerische Lösung der Kontinuumsgleichung}

Gleichungen des Typs (4.4) lassen sich nur in wenigen Fällen analytisch lösen, meist müssen die Lösungen numerisch berechnet werden. Das in dieser Arbeit verwendete Lösungsverfahren wurde bereits zur Simulation des Schichtwachstums von metallischen Gläsern [May97; May99; May00; Rai00; Str04; Str06] und deren Ionenbestrahlung [Vau07a; Vau07b; Vau08] verwendet. Es beruht auf einem von S. G. Mayr in C++ geschriebenen Computercode, in dem Integration und Differentiation durch Diskretisierung von Ort und Zeit approximiert werden [May97]. Die Ortsdiskretisierung ist über ein kubisches Gitter mit Gitterkonstante $\Delta x=1 \mathrm{~nm}, 400 \times 400$ Gitterplätzen und periodischen Randbedingungen in $x y$-Ebene realisiert, während die Zeit durch endliche Zeitschritte $\Delta t=25 \mu$ s diskretisiert wird. Die numerische Integration erfolgt durch das Euler-Verfahren [Pre02], das für hinreichend kleine Quotienten aus Orts- und Zeitschrittweite $\Delta t / \Delta x$ stabil ist.

Das Rauschen und die Depositionsrate sind in einer Zufallsvariable $\xi=\kappa \sqrt{2 R \Delta t} / \Delta x$ implementiert, wobei $\kappa$ gleichmäßig mit Standardabweichung 1 im Intervall $[0, \sqrt{12}]$ verteilt ist. Dazu wird in jedem Integrationsschritt mit Hilfe des Zufallszahlengenerators ran2 [Pre02] eine gleichverteilte Zufallszahl zwischen null und eins erzeugt und anschließend entsprechend der Rauschstärke und der Depositionsrate skaliert.

Die Parameter für die numerische Lösung von Gl. (4.8) sind in Tab. (4.1) aufgelistet. Dabei ist nur die effektive Hamaker-Konstante $A$ variiert worden ${ }^{3}$, die anderen Werte ba-

\footnotetext{
${ }^{3}$ Gezeigt werden nur Lösungen für die Paramter $A=1,6$ und $A=-1,6$. Diese Werte für $A$ sind im Vergleich zu anderen Systemen (vgl. z. B. [Bec03]) sehr hoch, dennoch liefern sie eine gute Übereinstimmung mit experimentellen Daten (vgl. Abs. 7).
} 


\begin{tabular}{|c|c|c|c|c|c|}
\hline $\begin{array}{c}\mathrm{A} \\
(\mathrm{nN} \mathrm{nm})\end{array}$ & $\begin{array}{c}\epsilon \\
\left(\mathrm{nN} \mathrm{nm}^{7}\right)\end{array}$ & $\begin{array}{c}\gamma \\
(\mathrm{nN} / \mathrm{nm})\end{array}$ & $\begin{array}{c}D \Omega \\
\left(\mathrm{nm}^{5} / \mathrm{nN} \mathrm{s}\right)\end{array}$ & $\begin{array}{c}\mathrm{d} \\
(\mathrm{nm})\end{array}$ & $\begin{array}{c}\mathrm{R} \\
\left(\mathrm{nm}^{4} / \mathrm{s}\right)\end{array}$ \\
\hline $\pm 1,6$ & 0,0006 & 0,04 & 3000 & 0,55 & 0,001 \\
\hline
\end{tabular}

Tabelle 4.1: Parameter zur Lösung der Kontinuumsgleichung (4.8).

sieren auf Literaturdaten oder sind wie folgt grob abgeschätzt: Für die Oberflächenspannung findet man in [LeG00] $\gamma=42 \mathrm{mN} / \mathrm{m}$. Für $\epsilon$ wird der Wert von Polystyrol auf $\mathrm{SiO}_{2}$ $\epsilon=6 \times 10^{-76} \mathrm{~J} / \mathrm{m}^{6}$ aus [Bec03] verwendet. Für den Radius $d$ wird als untere Grenze die mittlere Ausdehnung der Monomerbausteine eines vergröberten PC-Modells eingesetzt [Abr03], daraus ergibt sich das Teilchenvolumen unter der Annahme kugelförmiger Partikel zu $\Omega \approx 0,7 \mathrm{~nm}^{3}$. Die Rauschstärke erhält man aus $R=0,5 I \Omega \approx 0,001 \mathrm{~nm}^{4} / \mathrm{s}$ mit der aus dem Experiment bekannten Aufdampfrate $I=3 \AA / \mathrm{min}$ (vgl. Abs. 3.1.2). Um den Diffusionsterm $D$ abzuschätzen, der nach Mullins durch $D=D_{s} \Omega \nu /\left(k_{B} T\right)$ gegeben ist [Mul57], muss man im Wesentlichen den Oberflächendiffusionskoeffizienten $D_{s}$ ermitteln. Geht man davon aus, dass nur Teilchen in der obersten Lage zur Oberflächendiffusion beitragen, bleibt einem gerade deponierten Teilchen etwa soviel Zeit zu diffundieren, bis genug weiteres Material aufgedampft wurde, so dass es bedeckt ist - bei der gegebenen Aufdampfrate also $t \approx 400$ s. Typische Strukturgrößen im Experiment betragen $l \approx 100 \mathrm{~nm}$. Diese Größe muss in der vorgegeben Zeit gebildet werden und man kann den Diffusionskoeffizienten durch $D_{s} \approx l^{2} / t \approx 25 \mathrm{~nm}^{2} / \mathrm{s}$ abschätzen [Kru99]. Das Substrat befindet sich bei Raumtemperatur, somit ist $k_{B} T \approx 25 \mathrm{meV}$. Mit einer Teilchendichte von $1 / \mathrm{nm}^{2}$ erhält man: $D \Omega=D_{s} \Omega^{2} \nu /\left(k_{B} T\right) \approx 3000 \mathrm{~nm}^{5} / \mathrm{nNs}$.

Um eine bessere Vergleichbarkeit mit den Experimenten zu erreichen, bei denen größere Bereiche einer Oberfläche untersucht werden, kann man das gewonnene Höhenprofil reskalieren. Dies kann man als eine implizite Art der Parameteroptimierung betrachten, die dadurch gerechtfertigt ist, dass alle Parameter nur bei verschiedenen räumlichen Ableitungen - auch höherer Ordnung - von $h$ auftreten. Somit ist eine anschließende Reskalierung identisch mit einer vorherigen Anpassung der Startparameter. Im einfachsten Fall kann eine solche Reskalierung isotrop sein, d. h. horizontal und vertikal wird das System mit den gleichen Faktoren reskaliert. Eine allgemeinere Art der Reskalierung ist die affine Transformation: $\vec{x} \rightarrow b \vec{x}$ und $h \rightarrow b^{\alpha^{\prime}} h$ mit den Konstanten $b$ und $\alpha^{\prime}$. Um die Struktur der Oberfläche, vor allem von Fluktuationen auf kleinen Skalen, zu erhalten, kann man, wie im Folgenden verwendet, für die Konstante $\alpha^{\prime}$ den Rauigkeitsexponenten $\alpha$ wählen, wodurch die Reskalierung selbstaffin und somit strukturerhaltend wird (vgl. [Bar95; May99]). Die Strukturbildung auf kleinen Längenskalen wird durch die konkurrierenden Terme der Oberflächendiffusion und des Rauschens bestimmt und für kleine Abstände kann man aus dem Skalenverhalten der Höhendifferenzkorrelationsfunktion $H(r) \propto r^{2 \alpha}$ (für $r<<R_{c}$, vgl. Gl. (3.6)) den Rauigkeitsexponenten bestimmen [May99]. Der Parameter $b$ ist frei wählbar und kann so angepasst werden, dass die Bildgröße entsprechend groß genug ist. Auf die Bestimmung von $b$ wird kurz in Abs. 7 eingegangen.

Die Auswertung der simulierten Höhenprofile und deren Reskalierung erfolgt in IDL. Neben der visuellen Darstellung werden die Rauigkeit (vgl. Gl. (3.2)), die Höhendifferenzkorrelationsfunktion und damit der Rauigkeitsexponent $\alpha$ berechnet. 


\section{Molekulardynamik Simulationen}

Dieses Kapitel soll in die verwendeten Molekulardynamik (MD) Simulationen einführen. Mit den MD Simulationen soll der Einfluss von Oberflächen auf die Dynamik dünner Polymerfilme untersucht werden. Dazu werden einerseits eine einzelne Polymerkette auf der Oberfläche einer Simulationszelle aus denselben Polymeren mit den Ketten im Inneren und andererseits die oberflächennahen Ketten im Vergleich zu den zentralen Ketten untersucht.

\subsection{Grundlagen der MD Simulation}

Die (klassische) MD Simulation ist eine Methode zur Berechnung der Gleichgewichtsund Transporteigenschaften eines klassischen Vielteilchenproblems. Klassisch heißt dabei, dass Quanteneffekte nicht beachtet werden und als Grundlage die Newtonschen Bewegungsgleichungen verwendet werden. Die Kräfte auf ein Teilchen $i$ werden über $\vec{F}_{i}=-\nabla_{\vec{r}_{i}} U(\{\vec{r}\})$ aus dem Potential, bzw. der potentiellen Energie $U$ numerisch berechnet, wobei $\{\vec{r}\}$ die Menge der Teilchenpositionen angibt. Die Wahl und Parametrisierung des Potentials ist somit ein entscheidender Punkt bei MD Simulationen. Um die Berechnung der Kräfte zu beschleunigen, ist es bei kurzreichweitigen Potentialen zudem oft sinnvoll, Wechselwirkungen nur bis zu einem gewissen Abstand $r_{c}$ zu berechnen, da die Wechselwirkungsstärke bei größeren Abständen sehr klein ist. Um Potentialsprünge bei $r_{c}$ zu vermeiden, wird das Potential so verschoben dass $U\left(r_{c}\right) \equiv 0$ gilt.

Sind alle Kräfte berechnet, lassen sich die Bewegungsgleichungen numerisch durch Integration mittels geeigneter Algorithmen (wie z. B. das Euler Prädiktor-KorrektorVerfahren, der Verlet-Algorithmus, etc. [Kre89; All89]) lösen und für alle Teilchen werden die neuen Positionen und Geschwindigkeiten berechnet. Geeignet heißt dabei, dass der Algorithmus schnell, energie- und impulserhaltend und wenig speicheraufwendig sein soll und einen genügend großen Zeitschritt erlauben soll [All89]. Ein weiterer wichtiger Punkt ist die Wahl des Ensembles. Das System kann an ein Wärmebad gekoppelt werden (konstante Temperatur $T$ ) oder die innere Energie $E$ bleibt konstant, die Teilchenzahl $N$ bleibt oftmals erhalten und man kann das Volumen $V$ oder den Druck $p$ konstant halten. Es gibt also verschiedene Ensembles, z. B. das mikrokanonische Ensemble (NVE: mit konstanter Teilchenzahl, konstantem Volumen V und konstanter innerer Energie), das kanonische Ensemble (NVT), das isotherm-isobare (oder auch kanonisch-harmonische) Ensemble (NpT) und viele andere $(\mathrm{NpH}, \mu \mathrm{VT}, \ldots)$. Zur Erhaltung dieser Größen werden Regler (z. B. Nosé-Hoover [Nos84; Hoo85; Hoo86]) eingesetzt, die die Bewegungsgleichungen z. B. durch Einführung eines Reibungskoeffizienten, der die Kopplung an ein Wärmebad beinhaltet, erweitern oder geeignete Reskalierungen verwenden.

An dieser Stelle soll aber keine ausführliche Einführung in MD Simulationen oder Potentialentwicklungen gegeben werden, dafür gibt es genügend Literatur (z. B. [All89; Rap04]). 


\subsection{Simulation eines Polymerfilms}

Für alle MD Simulationen in dieser Arbeit wird das frei verfügbare LAMMPS ${ }^{1}$ Paket in der Version vom 12. April 2006 verwendet [Pli95], das für den effizienten Einsatz auf Parallelrechnersystemen ausgelegt ist. Wenn nicht anders angegeben, werden sie im NpT Ensemble mit Nosé-Hoover Temperatur- und Druckkontrolle und dem VelocityVerlet Integrationsalgorithmus [Win06; All89] mit einem Zeitschritt $\Delta t=0,005 \tau$ (vgl. S. 35) durchgeführt. Dieser Algorithmus beruht auf dem Verlet Algorithmus [Ver67], bei dem die Ortskoordinaten eines Teilchens im nächsten Zeitschritt $\vec{x}_{i}(t+\Delta t)$ aus der aktuellen Position $\vec{x}_{i}(t)$ und der des Zeitschritts zuvor $\vec{x}_{i}(t-\Delta t)$ berechnet werden. Statt der Position des vorherigen Zeitschritts wird beim Velocity-Verlet Algorithmus die aktuelle Geschwindigkeit $\dot{\vec{x}}_{i}(t)$ verwendet:

$$
\begin{aligned}
& \vec{x}_{i}(t+\Delta t)=\vec{x}_{i}(t)+\dot{\vec{x}}_{i}(t) \Delta t+\frac{\vec{F}_{i}(t)}{2 m_{i}}(\Delta t)^{2} \\
& \dot{\vec{x}}_{i}(t+\Delta t)=\dot{\vec{x}}_{i}(t)+\frac{\vec{F}_{i}(t)+\vec{F}_{i}(t+\Delta t)}{2 m_{i}} \Delta t
\end{aligned}
$$

\subsubsection{Das Potential}

Für die Modellierung von Polymeren in Simulationen sind neben der Kettenstruktur die Flexibilität und die Abstoßung von Monomeren, die sich nicht überlappen dürfen (,excluded volume“), entscheidend, außerdem dürfen sich die Ketten nicht durchschneiden. Aus diesem Grund bietet sich ein Modell aus miteinander durch (harmonische oder anharmonische) Federn verbundener Kugeln an, ein sog. Federmodell ${ }^{2}$. Kremer und Grest haben ein Modell vorgeschlagen [Kre90], das seitdem für viele Polymersimulationen verwendet worden ist (z. B. [Ben98; Var02; Muk06; Des06]). Dabei wechselwirken alle Teilchen über ein abgeschnittenes und verschobenes (s. o.) Lennard-Jones (LJ) Potential miteinander, wie es in Abb. 5.1(schwarze Linie) gezeigt ist:

$$
U_{L J}(r)= \begin{cases}4 \epsilon\left[(\sigma / r)^{12}-(\sigma / r)^{6}\right]-U_{L J}\left(r_{c}\right) & \text { für } r \leq r_{c} \\ 0 & \text { sonst }\end{cases}
$$

Je nach Wahl des Abschneideradius $r_{c}$ kann das LJ Potential rein repulsiv sein $\left(r_{c}=\right.$ $\left.r_{\text {min }}=2^{1 / 6} \sigma\right)$, wie in [Kre90; Kop97] oder einen kleinen attraktiven Anteil beinhalten. Wolfgardt et al. haben gezeigt, dass ein Modell ohne einen attraktiven Anteil einen negativen thermischen Ausdehnungskoeffizienten liefert [Wol96], daher wird in dieser Arbeit $r_{c}=2 r_{\min }=2 \times 2^{1 / 6} \sigma$ gewählt. Zusätzlich sind benachbarte Teilchen entlang einer Kette über das FENE ${ }^{3}$ Potential verbunden (siehe Abb. 5.1(blaue Symbole)), das den Zusammenhalt der Kette gewährleisten soll:

$$
U_{F E N E}(r)=-\frac{k}{2} R_{0}^{2} \ln \left[1-\left(\frac{\mathrm{r}}{\mathrm{R}_{0}}\right)^{2}\right]
$$

\footnotetext{
${ }^{1}$ LAMMPS für Large-scale Atomic / Molecular Massively Parallel Simulator.

${ }^{2}$ Im Englischen bead-spring model.

${ }^{3}$ FENE für Finitely Extendable Nonlinear Elastic.
} 


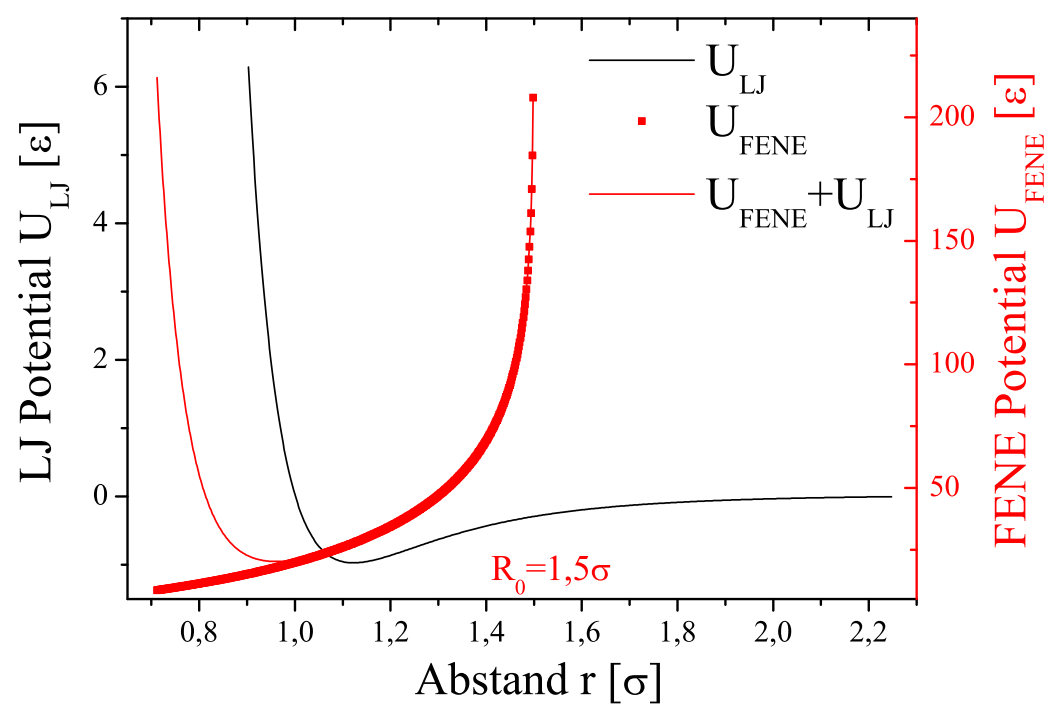

Abbildung 5.1: Zwischen allen Teilchen wirkt ein abgeschnittenes LJ Potential (schwarze Linie). Zusätzlich sind Nachbarn entlang einer Polymerkette durch das FENE Potential (rote Symbole) verbunden. Das Zusammenspiel beider Potentiale ist als rote Linie dargestellt. Durch die logarithmische Divergenz des FENE Potentials bei $R_{0}$ können sich Bindungen nicht weiter ausdehnen und der starke repulsive Anteil des LJ Potentials verhindert, dass sich Teilchen überlappen können (,excluded volume“).

Die Parameter sind dabei mit $k=30 \epsilon / \sigma^{2}$ und $R_{0}=1,5 \sigma$ so gewählt, dass Schneiden von Ketten und hochfrequente Moden vermieden werden [Ben98]. $R_{0}$ definiert die maximale Ausdehnung einer Bindung, da an dieser Stelle der logarithmische Term divergiert. Aus dem Zusammenspiel des LJ Potentials und des FENE Potentials resultiert für benachbarte Teilchen entlang einer Kette der bevorzugte Bindungsabstand ${ }^{4}$ zu 0,96 $\sigma$. Eine schematische Darstellung der vorhandenen Wechselwirkungen ist in Abb. 5.2 gezeigt.

Da es hier nicht um die Simulation eines bestimmten Polymers geht, werden im Folgenden alle Ergebnisse in LJ Einheiten angegeben, wobei die Längenskala durch $\sigma$ und die Energieskala durch $\epsilon$ festgelegt ist, wodurch die Temperatur in $\epsilon / k_{B}\left(k_{B}=1\right)$ und die Zeit in $\tau=\sqrt{m \sigma^{2} / \epsilon}(m=1)$ gemessen werden. Tab. 5.1 gibt einen Überblick über diese und weitere LJ Einheiten. Für einen Vergleich mit realen Systemen, reskaliert man die Ergebnisse mit entsprechenden Werten für $\sigma, \epsilon$ und $m$. Z. B. findet man für Polyethylen [Kre90]: $\sigma \approx 5 \AA, \epsilon \approx 39 \mathrm{meV}$ und $m \approx 28 \mathrm{~g} / \mathrm{mol}$, womit der gewählte Zeitschritt von $\Delta t=0,005 \tau$ etwa 6,8 fs entspricht. Dieser Wert erscheint für atomistische Simulationen zunächst sehr hoch, wenn hochfrequente Moden vermieden werden sollen. Allerdings werden wegen der Vergröberung des Federmodells Seitengruppenschwingungen und schnelle C-H Schwingungen nicht betrachtet, sondern nur die langsameren Schwingungen der Hauptkette $\left(\approx 4 \times 10^{-14}\right.$ s), so dass der Zeitschritt immer noch etwa eine Größenordnung kleiner ist.

\footnotetext{
${ }^{4}$ Da dieser Abstand nicht mit dem Gleichgewichtsabstand des LJ Potentials zusammenpasst, kristallisiert das System nicht, bzw. nur schwer.
} 


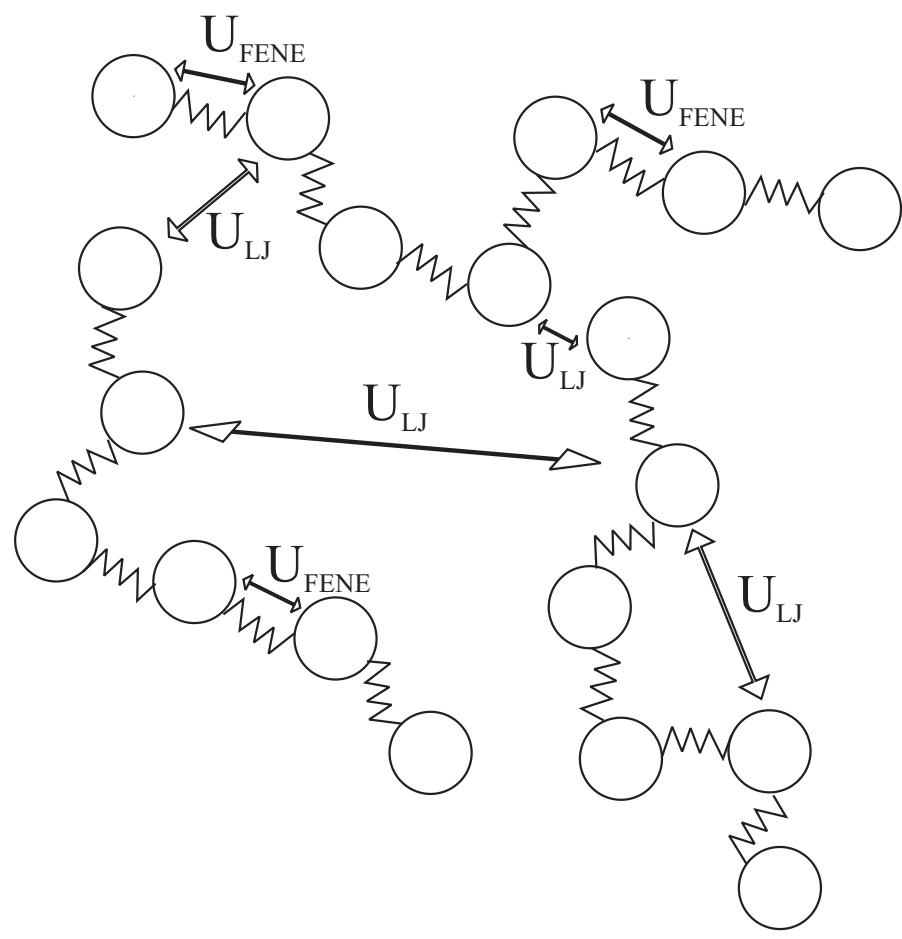

Abbildung 5.2: Schematische Darstellung der vorhandenen Wechselwirkungen in der MD Simulation. Die Polymerketten werden durch über Federn verbundene Kugeln simuliert. Man unterscheidet zwischen der Wechselwirkung zwischen beliebigen Teilchen und der Bindung entlang einer Kette.

\subsubsection{Herstellung und Vorbereitung der Simulationszellen}

Für die Herstellung der Zellen wird ein Verfahren in zwei Varianten verwendet, wie es von Kremer und Grest vorgeschlagen worden ist [Kre90]. Die eine Variante startet zunächst mit periodischen Randbedingungen in allen Richtungen (Variante 1), während die andere von vornherein mit freien Oberflächen in z-Richtung startet (Variante 2), d. h. es werden periodische Randbedingungen nur in $\mathrm{x}$ - und $\mathrm{y}$-Richtung verwendet. Beide Varianten werden später untersucht. Für die Simulation einer einzelnen Kette auf der Oberfläche wird nur Variante 1 betrachtet, während für die Untersuchungen des Einflusses der Oberflächen auf die Dynamik beide Varianten verwendet werden. Wie sich herausstellt (vgl. Anhang Abs. 11.4), liefern beide Herstellungsvarianten vergleichbare Ergebnisse. Die Simulation mit freien Oberflächen ist vergleichbar mit einem unendlich ausgedehnten dünnen freistehenden Film im Vakuum. Der prinzipielle Ablauf ist für beide Varianten ähnlich und wird im Folgenden aufgelistet:

\begin{tabular}{|c|c|c|c|c|c|c|}
\hline Länge & Energie & Zeit & Kraft & Geschwindigkeit & Druck & Temperatur \\
\hline$\sigma$ & $\epsilon$ & $\tau=\sigma(m / \epsilon)^{0,5}$ & $\epsilon / \sigma$ & $(\epsilon / m)^{0,5}$ & $\epsilon / \sigma^{3}$ & $\epsilon / k_{B}$ \\
\hline
\end{tabular}

Tabelle 5.1: Übersicht über LJ Einheiten. 
Startkonfiguration: Die Ketten werden nacheinander mit fester Bindungslänge zufällig in einer rechteckigen Box, deren Größe durch die vorgegebe Dichte definiert ist, platziert. An dieser Stelle sind Überlappungen verschiedener Ketten erlaubt, da der Platzierungsalgorithmus sonst zu ineffizient wäre. Nur in Variante 2 mit freien Oberflächen gibt es die Beschränkung, dass eine Kette die Oberfläche nicht schneiden darf. Solche Platzierungsversuche werden verworfen und eine neue Kette wird platziert. Typischerweise werden in dieser Arbeit 1730 Ketten aus jeweils 30 Kugeln verwendet, d. h. insgesamt 51900 Teilchen und 50170 FENE Bindungen.

Abbau von Überlagerungen: Da bei der zufälligen Platzierung der Ketten sehr kleine Abstände mit Überlagerungen entstehen können, die durch die starke Repulsion des LJ Potentials zu sehr hohen Kräften und damit zur Explosion der Zelle führen können, müssen diese Überlagerungen zunächst abgebaut werden. Dazu wird für kurze Zeit $\left(t=1 \times 10^{4} \Delta \mathrm{t}\right)$ bei Druck $p=1 \epsilon / \sigma^{3}$ und Temperatur $T=1 \epsilon / \mathrm{k}_{\mathrm{B}}$ ein elastischeres Potential angelegt, das auch bei kleinen Abständen nicht zu stark ansteigt:

$$
U(r)= \begin{cases}A\left[1+\cos \left(\frac{\pi r}{r_{c, s}}\right)\right] & \text { für } r<r_{c, s} \\ 0 & \text { sonst }\end{cases}
$$

Dabei gibt $r_{c, s}$ den Abstand an, bis zu dem die Teilchen auseinandergedrückt werden. Die Stärke der Abstoßung wächst mit der Zeit von $A=4$ auf $A=100$ an.

In Variante 2 werden rein repulsive LJ Wände über und unter der Zelle platziert, um ein Auseinanderlaufen der Oberflächen zu verhindern. Außerdem laufen diese Simulationen im NVT statt im NpT Ensemble.

Anschließend werden alle Geschwindigkeiten auf Null gesetzt und in zufälliger Richtung neu zufällig verteilt entsprechend der Temperatur initialisiert, um die Bildung gerichteter Geschwindigkeiten zu verhindern. Es folgt ein kurzer Lauf mit FENE Potential und einem rein repulsiven LJ Potential für weitere $t=1 \times 10^{4} \Delta \mathrm{t}$ bei Druck $p=1 \epsilon / \sigma^{3}$ (nur Variante 1, da Variante 2 im NVT Ensemble) und Temperatur $T=1 \epsilon / \mathrm{k}_{\mathrm{B}}$. Bei dieser Temperatur liegt das System in der Schmelze vor und eine statistische Bewegung der Teilchen ist gewährleistet. Danach wird das Potential wie in den Gl. (5.2) und (5.3) definiert angelegt und das System für $t=$ $8 \times 10^{4} \Delta$ t relaxiert. Nach einer kurzen Einschwingzeit bleibt die Gesamtenergie innerhalb von ca. $0,5 \%$ konstant.

In Variante 2 mit Oberflächen werden nun die LJ Wände entfernt und das System für weitere $t=1 \times 10^{6} \Delta \mathrm{t}$ im NVT Ensemble relaxiert.

Ausschalten der Druckkontrolle: Bei Simulationen mit freien Oberflächen führt die Druckkontrolle aufgrund der Oberflächenspannung zur Minimierung der Oberfläche und damit zu einem Zusammenziehen der gesamten Zelle, daher muss sie deaktiviert werden. Eine Druckkontrolle arbeitet also nur mit periodischen Randbedingungen, da ein definiertes Volumen für die Regelung benötigt wird [Rap04]. 

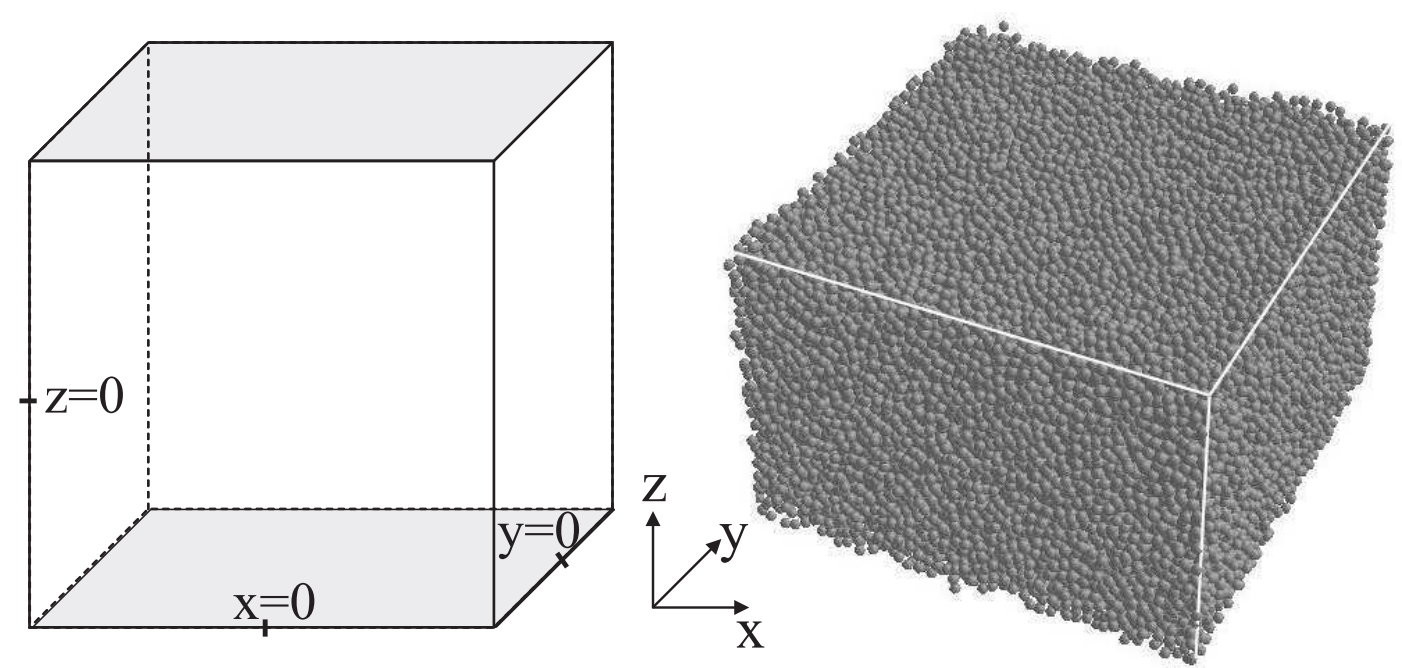

Abbildung 5.3: Schematische Darstellung (links) und Momentaufnahme (rechts) einer Simulationszelle. In x- und y-Richtung wirken periodische Randbedingungen, während die Zelle in z-Richtung offen ist und somit ein quasi unendlich ausgedehnter dünner freistehender Film simuliert wird.

Variante 1: Der Druck wird zunächst von $p=1 \epsilon / \sigma^{3}$ bei $T=1 \epsilon / \mathrm{k}_{\mathrm{B}}$ in $t=1 \times 10^{5} \Delta \mathrm{t}$ auf $p=0 \epsilon / \sigma^{3}$ reduziert und das System für mindestens $t=6 \times 10^{6} \Delta \mathrm{t}$ bei $T=1 \epsilon / \mathrm{k}_{\mathrm{B}}$ relaxiert. Anschließend werden die periodischen Randbedingungen in z-Richtungen fallen gelassen. Bei Ketten, die diese Fläche schneiden, werden die entsprechenden Bindungen gelöscht, die Ketten werden also zerschnitten. Kettensegmente mit weniger als drei Teilchen werden aus der Box entfernt, da solche Segmente manchmal auch bei niedrigen Temperaturen abdampfen. Die Druckkontrolle wird deaktiviert und das System wird für mindestens $t=2 \times 10^{6} \Delta$ t relaxiert. Dabei bleiben die $\mathrm{x}$ - und y-Dimension unverändert.

Variante 2: Es wird vom NVT in das NpT Ensemble mit deaktivierter Druckkontrolle gewechselt, d. h. x- und y-Ausdehnung bleiben konstant, alle Geschwindigkeiten noch einmal reinitialisiert und das System für mindestens $t=6 \times 10^{6} \Delta \mathrm{t}$ bei $T=1 \epsilon / \mathrm{k}_{\mathrm{B}}$ relaxiert. Die Gesamtenergie bleibt in dieser Zeit innerhalb von $<1 \%$ konstant.

Abschließende Präparation: Nach dieser Relaxation wird das System entweder zur Bestimmung der Glasübergangstemperatur mit verschiedenen Raten zwischen $\frac{9 \epsilon / \mathrm{k}_{\mathrm{B}}}{10^{6} \Delta \mathrm{t}}$ und $\frac{9 \epsilon / \mathrm{k}_{\mathrm{B}}}{10^{8} \Delta \mathrm{t}}$ abgekühlt oder aber zur Vorbereitung der eigentlichen Simulationsläufe schrittweise abgekühlt, zunächst in $t=1 \times 10^{5} \Delta \mathrm{t}$ von $T=1 \epsilon / \mathrm{k}_{\mathrm{B}}$ auf $T=0,4 \epsilon / \mathrm{k}_{\mathrm{B}}$, wo das System für weitere $t=5 \times 10^{6} \Delta$ t verweilt und dann in $t=1 \times 10^{5} \Delta \mathrm{t}$ auf $T=0,2 \epsilon / \mathrm{k}_{\mathrm{B}}$, wo es erneut für $t=3 \times 10^{6} \Delta \mathrm{t}$ gehalten wird. Dieser Punkt dient als Tieftemperatur-Startpunkt.

Ein Bild der so hergestellten Zellen ist in Abb. 5.3 schematisch (links) und als Momentaufnahme (rechts) gezeigt. 


\subsubsection{Simulation einer einzelnen Kette auf der Oberfläche}

Zunächst wird die Zelle auf die gewünschte Temperatur gebracht. Für diese Untersuchungen werden im Wesentlichen $T=0,4 \epsilon / \mathrm{k}_{\mathrm{B}}$ und $T=0,44 \epsilon / \mathrm{k}_{\mathrm{B}}$ betrachtet. Anschließend wird eine lineare Kette gerade auf der oberen Oberfläche positioniert. Die Simulation startet (Zeitpunkt $t_{0}$ ) und es werden mindestens alle $t=1 \times 10^{5} \Delta$ t Momentaufnahmen der einzelnen Kette und alle $t=1 \times 10^{6} \Delta$ t Momentaufnahmen der gesamten Zelle gemacht.

Die Positionen und Geschwindigkeiten werden anschließend mit IDL ausgewertet. Dabei werden statische und dynamische Größen wie der Gyrationsradius, der End-zuEnd Abstand, der Schwerpunkt sowie mittlere Verschiebungsweiten aller Monomere, der Schwerpunkte und der Kettenmittelpunkte berechnet (vgl. Abs. 2.2).

\subsubsection{Simulation des Einflusses der Oberflächen auf die Dynamik}

Vom Tieftemperatur-Startpunkt wird die Zelle innerhalb von $t=1 \times 10^{5} \Delta$ t auf die gewünschte Temperatur geheizt und dort für mindestens $t=8 \times 10^{6} \Delta$ t gehalten, bis die Gesamtenergie innerhalb des Rauschens konstant bleibt und nicht weiter abfällt.

Nun wird das System von dieser Startzeit $t_{0}$ aus für mindestens $t=1 \times 10^{8} \Delta$ t beobachtet. Alle $t=1 \times 10^{6} \Delta \mathrm{t}$ wird eine Momentaufnahme aller Positionen und Geschwindigkeiten aufgenommen. Außerdem werden alle $t=500 \Delta$ t die Temperatur, Gesamtenergien (innere Energie, kinetische Energie, Bindungsenergie), Druck und Volumen ausgegeben.

Um den Einfluss der Oberflächen zu untersuchen, werden 17 verschiedene Temperaturen im Bereich 0,39 $\epsilon / \mathrm{k}_{\mathrm{B}} \leq T \leq 0,62 \epsilon / \mathrm{k}_{\mathrm{B}}$ simuliert. Zum Vergleich wird eine Zelle mit periodischen Randbedingungen in allen Richtungen untersucht. Um Effekte durch die begrenzte Größe der Zelle auszuschließen, wird außerdem eine doppelt so große Zelle simuliert. Des Weiteren wird der Einfluss der letzten Relaxationszeit auf das System untersucht.

Die Auswertung erfolgt anschließend in IDL. Aus den Positionen aller Teilchen werden die in Abs. 2.2 Größen und zeitliche Fluktuationen im Gyrationsradius und End-zu-End Abstand berechnet und entsprechend ihrer z Koordinate gemittelt.

\subsubsection{Verwendete Computer-Cluster}

Die Simulationen dieser Arbeit sind hauptsächlich auf dem Woodcrest-Cluster mit Intel Xeon Prozessoren der GWDG ${ }^{5}$ entstanden. Typischerweise werden die Simulationen parallel auf 16 Prozessoren durchgeführt. Neben dem Woodcrest-Cluster sind auch das Parallelrechnersystem IBM pSeries690 der GWDG, der Rocks-GoeGrid Cluster der Fakultät für Physik sowie der Computercluster der Arbeitsgruppe Mayr verwendet worden. Da alle Ergebnisse im Rahmen der statistischen Fehler identisch sind, wird im Folgenden nicht weiter unterschieden, welche Simulationen auf welchen Rechnern gelaufen sind.

\footnotetext{
${ }^{5}$ GWDG für Gesellschaft für wissenschaftliche Datenverarbeitung mbH Göttingen.
} 



\section{Experimentelle Ergebnisse}

An dieser Stelle sollen die wesentlichen Ergebnisse der experimentellen Untersuchungen dieser Arbeit vorgestellt werden. Diese lassen sich in vier Abschnitte gliedern, wobei die strukturellen Eigenschaften und chemische Zusammensetzung der thermisch aufgedampften PC Schichten zuerst präsentiert werden. Es folgen die Untersuchungen der Topographien auf verschiedenen Substraten, wobei zunächst die Unterschiede direkt nach der Herstellung und anschließend die zeitliche Entwicklung der Topographien gezeigt werden. Am Ende werden erste Versuche zur lokalen Messung der mechanischen bzw. elastischen Eigenschaften der Proben vorgestellt.

\subsection{Struktur thermisch aufgedampfter PC Schichten}

\subsubsection{Zusammensetzung und Struktur}

Wie in Abs. 3.2.3 beschrieben werden Weitwinkelröntgenmessungen (Wellenlänge $\lambda_{C u-K_{\alpha}}=0,154 \mathrm{~nm}$ ) zur Strukturbestimmung verwendet. Abb. 6.1(a) zeigt eine solche Messung an einer $180 \mathrm{~nm}$ dicken thermisch aufgedampften PC Schicht hergestellt auf Si(100). Außerdem ist in dieser Abbildung noch eine Messung an einem leeren Si Substrat gezeigt. Beide Proben sind vor der Messung auf einen großen Si Halter aufgeklebt worden, um Reflexe vom Probentisch zu verhindern. Die PC Schicht weist ein breites Maximum um $2 \theta \approx 19^{\circ}$ auf. Bei $2 \theta=22,4^{\circ}$ ist ein zweites, schmaleres Maximum zu erkennen, das, da es auch auf dem leeren Substrat gemessen wird, dem Silizium zuzuschreiben ist. Die Lage passt zum Si(110) Reflex, der hier nur aufgrund der großen Blenden und der Verkippung messbar wird ${ }^{1}$. Unterhalb von $2 \theta \leq 11^{\circ}$ steigt die Intensität apparaturbedingt durch die feste Blendengeometrie und die damit zusammenhängende größere bestrahlte Fläche an. In Abb. 6.1(b) ist zum Vergleich eine Weitwinkelmessung an Massivproben aus PC nach verschiedener Temperaturbehandlung aus der Literatur gezeigt [Nek73]. Form und Breite der nicht-kristallisierten Proben stimmen mit der gemessenen Kurve überein.

Zur Charakterisierung der Bindungsstruktur werden wie in Abs. 3.2.1 beschrieben FTIR Spektren aufgenommen. Eine solche Messung ist in Abb. 6.2 für eine $85 \mathrm{~nm}$ dicke PC Schicht, die durch thermisches Aufdampfen auf rauem Aluminium Substrat hergestellt worden ist, gezeigt. Das Spektrum ist auf die maximale Absorptionsintensität (hier die C-O Bande), bzw. die maximale Amplitude, normiert. Zum Vergleich ist außerdem ein Spektrum aus der Literatur dargestellt [Sch64], das zum besseren Vergleich versetzt dargestellt ist. Durch die Normierung sind die Maximalamplituden beider Spektren gleich groß. Die einzelnen Absorptionsbanden können mit Hilfe von Literaturdaten den entsprechenden Bindungen zugeordnet werden [Fal97; Sch64]. Dies ist in Tab. 6.1

\footnotetext{
${ }^{1} \operatorname{Der} \mathrm{Si}(400)$ Reflex ist ca. $10^{6}$ mal stärker.
} 
(a)

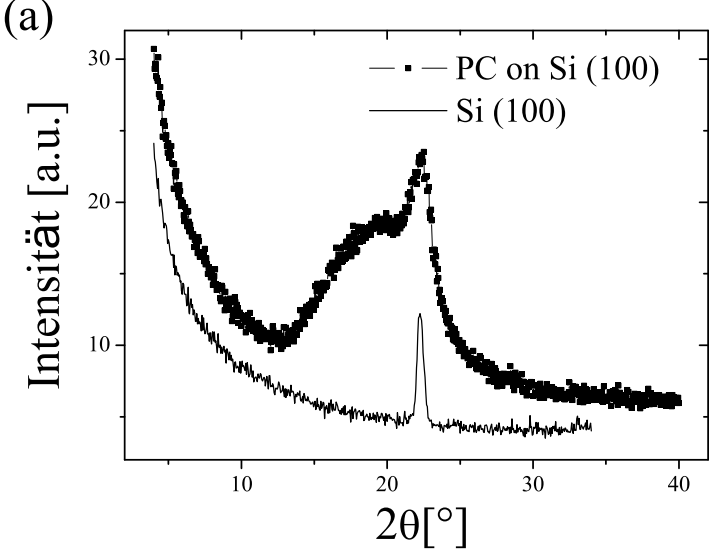

(b)

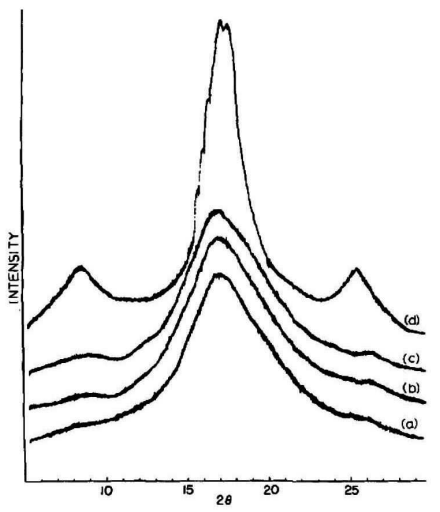

Abbildung 6.1: (a) Weitwinkelröntgenmessung einer $180 \mathrm{~nm}$ dicken thermisch aufgedampften PC Schicht hergestellt auf Si und eines leeren Si Substrats (Wellenlänge $\lambda_{C u-K_{\alpha}}=0,154 \mathrm{~nm}$ ). Neben einem breiten Maximum bei $2 \theta \approx 19^{\circ}$ erkennt man ein zusätzliches schmaleres Maximum bei $2 \theta=22,4^{\circ}$, das auch auf dem leeren Substrat zu sehen ist. (b) Weitwinkelmessung aus der Literatur [Nek73] für verschieden behandelte PC Massivproben ((a) unbehandelt, (b) 7 Tage $110{ }^{\circ} \mathrm{C}$, (c) 7 Tage $145^{\circ} \mathrm{C}$ und (d) kristallisiert).

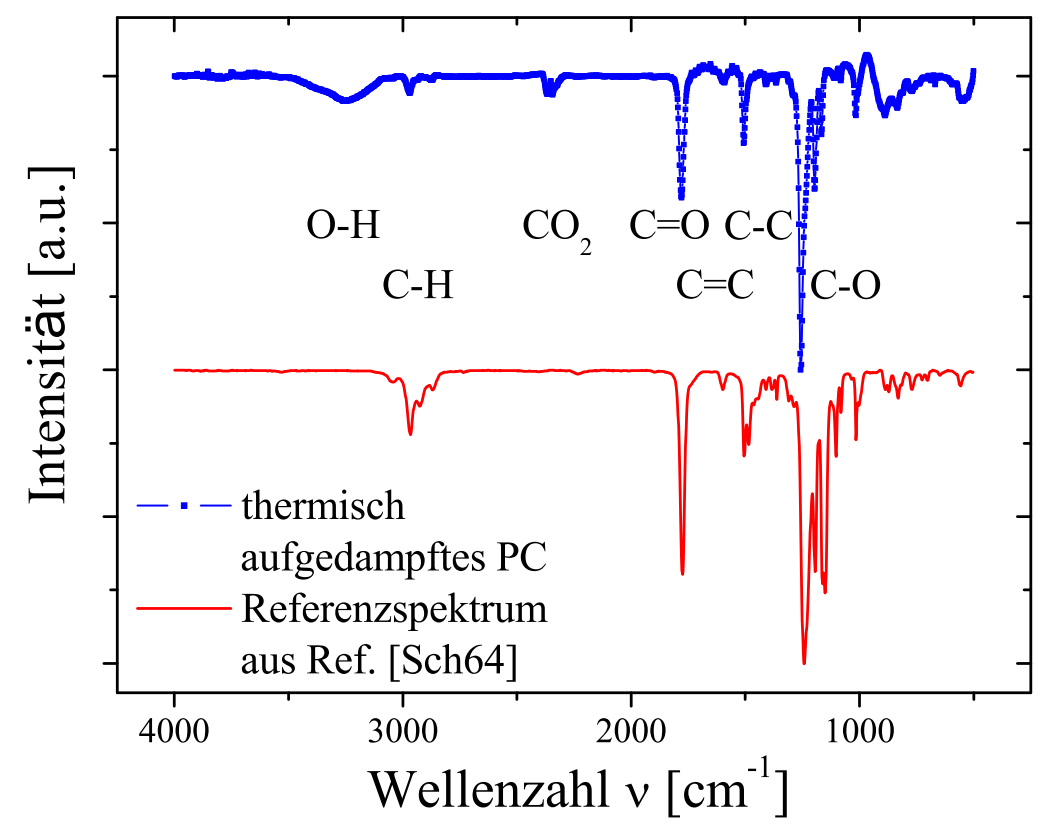

Abbildung 6.2: IR-Spektren einer $85 \mathrm{~nm}$ dicken thermisch aufgedampften PC Schicht hergestellt auf Aluminium und einer PC Massivprobe aus der Literatur [Sch64]. Die Absorptionskanten werden mit Hilfe von Literaturdaten den entsprechenden Bindungen zugeordnet [Fal97; Sch64] und in Tab. 6.1 verglichen. Beide Spektren stimmen in weiten Bereichen überein, auch wenn sich die Intensitäten einzelner Maxima unterscheiden. 


\begin{tabular}{|c|c|c|c|}
\hline $\begin{array}{c}\text { Wellenzahl } \\
\nu\left[\mathrm{cm}^{-1}\right]\end{array}$ & Bindung & $\begin{array}{c}\text { Intensität } \\
\mathrm{PC}, \text { Referenz }\end{array}$ & $\begin{array}{c}\text { Intensität } \\
\mathrm{PC}, \text { aufgedampft }\end{array}$ \\
\hline $1000-1300$ & $C-O$ & 1 & 1 \\
\hline $1450-1600$ & $C-C$ & 0,3 & 0,25 \\
\hline $1640-1680$ & $C=C$ & 0,1 & 0,05 \\
\hline $1690-1760$ & $C=O$ & 0,7 & 0,4 \\
\hline $2275-2400$ & $C O_{2}$ & 0 & 0,1 \\
\hline $2850-2960$ & $C-H$ & 0,2 & 0,05 \\
\hline $3200-3600$ & $O-H$ & 0 & 0,1 \\
\hline
\end{tabular}

Tabelle 6.1: Zuordnung und Vergleich der Absorptionskanten aus Abb. 6.2.

zusammengefasst. Die Bande bei $\nu \approx 2300 \mathrm{~cm}^{-1}$ kann $\mathrm{CO}_{2}$ zugeordnet werden und ist apparaturbedingt. Beide Spektren sind in qualitativ guter Übereinstimmung, alle Banden, die im Literaturspektrum auftauchen sind auch beim aufgedampften PC vorhanden, allerdings mit teilweise geringerer Intensität. Bei $\nu \approx 3200-3600 \mathrm{~cm}^{-1}$ tritt eine neue Bande auf, die zur O-H Bande einer Phenolschwingung gehört. Die Bindungsstruktur bleibt demnach weitestgehend intakt.

\subsubsection{Kettenlänge und Molmassenverteilung}

Um die Veränderung der Molmasse durch das thermische Aufdampfen zu analysieren, sind in Abb. 6.3 SEC Messungen von aufgedampftem PC und vom Ausgangsmaterial gezeigt. Beide Spektren sind auf ihre maximale Intensität normiert. Bei den angegebenen Molmassen muss beachtet werden, dass sie aufgrund der universellen Kalibrierung der Apparatur von der tatsächlichen Molmasse abweichen können. Das Ausgangsmaterial zeichnet sich durch ein breites Maximum bei hohen Molmassen $\left(M_{w} \approx 29000 \mathrm{~g} / \mathrm{mol}\right)$ aus. Bei niedrigen Molmassen sind außerdem einige kleine Maxima zu erkennen. Dagegen zeigt das aufgedampfte Material große Signale bei kleinen Molmassen und nur ein sehr schwaches Signal bei hohen Molmassen, das erst durch eine logarithmische Auftragung der Intensität, wie sie im Einsatz in Abb. 6.3 gezeigt ist, sichtbar wird. Es ist deutlich, dass sich die Molmasse und damit die Kettenlänge durch das Aufdampfen verringert.

Da die SEC Messungen gerade bei kleinen Molmassen durch die universelle Kalibrierung ungenau sind, zeigt Abb. 6.4 ein ESI Massenspektrum derselben Probe, die auch bei der SEC Messung verwendet worden ist. Da das Massenspektrometer maximal Masse-zu-Ladungs Verhältnisse von $m / z=2000 \mathrm{u}$ detektieren kann, können aus dieser Messung keine Aussagen über das Auftreten höherer Massen getroffen werden. Das Spektrum weist viele kleinere Maxima neben den größten Ausschlägen bei $m / z=954 \mathrm{u}$, $m / z=1248 \mathrm{u}$ und $m / z=780 \mathrm{u}$ auf. Im Anhang in Abs. 11.1 sind außerdem hochauflösende Massenspektren des FT-ICR-MS gezeigt, das eine Bestimmung der chemischen Zusammensetzung der einzelnen Maxima erlaubt. 


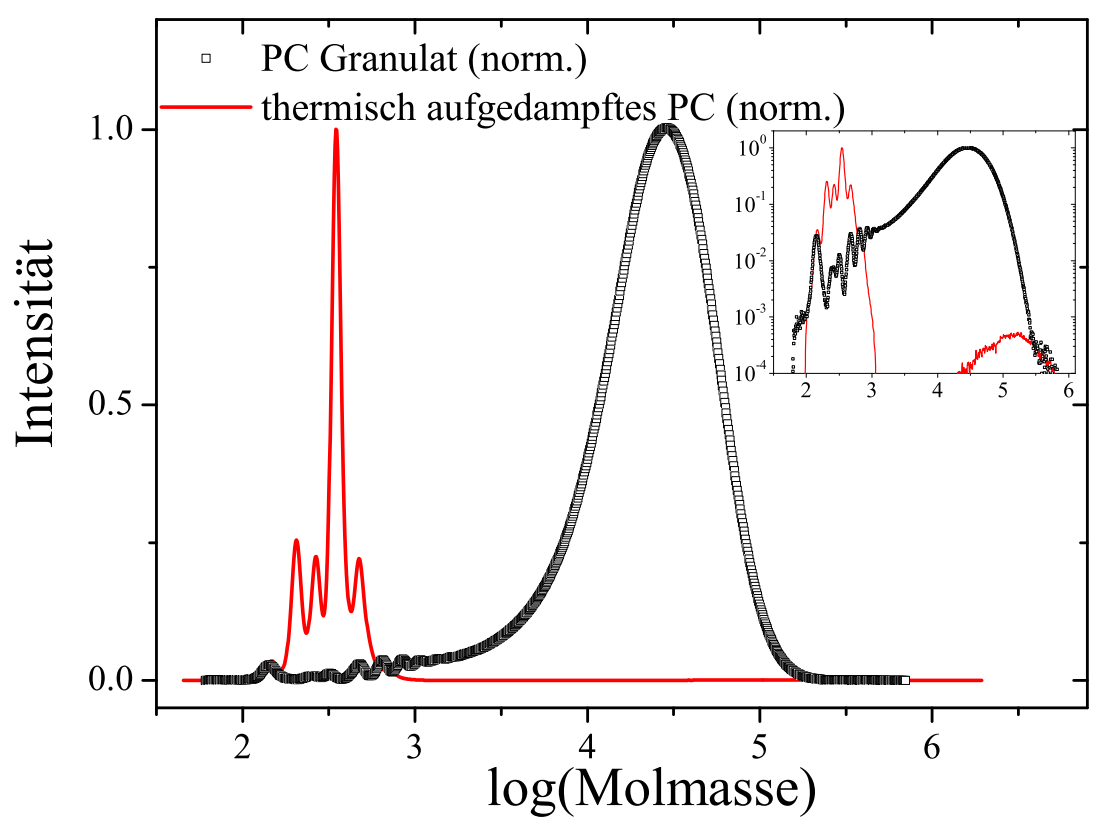

Abbildung 6.3: SEC Messungen an thermisch aufgedampftem PC und an PC Granulat (Ausgangsmaterial). Der Einsatz zeigt dieselben Daten mit logarithmisch aufgetragener Intensität, um das kleine Signal bei hohen Molmassen für das thermisch aufgedampfte Material sichtbar zu machen. Es ist zu erkennen, dass sich die Molmasse durch das Aufdampfen verringert.

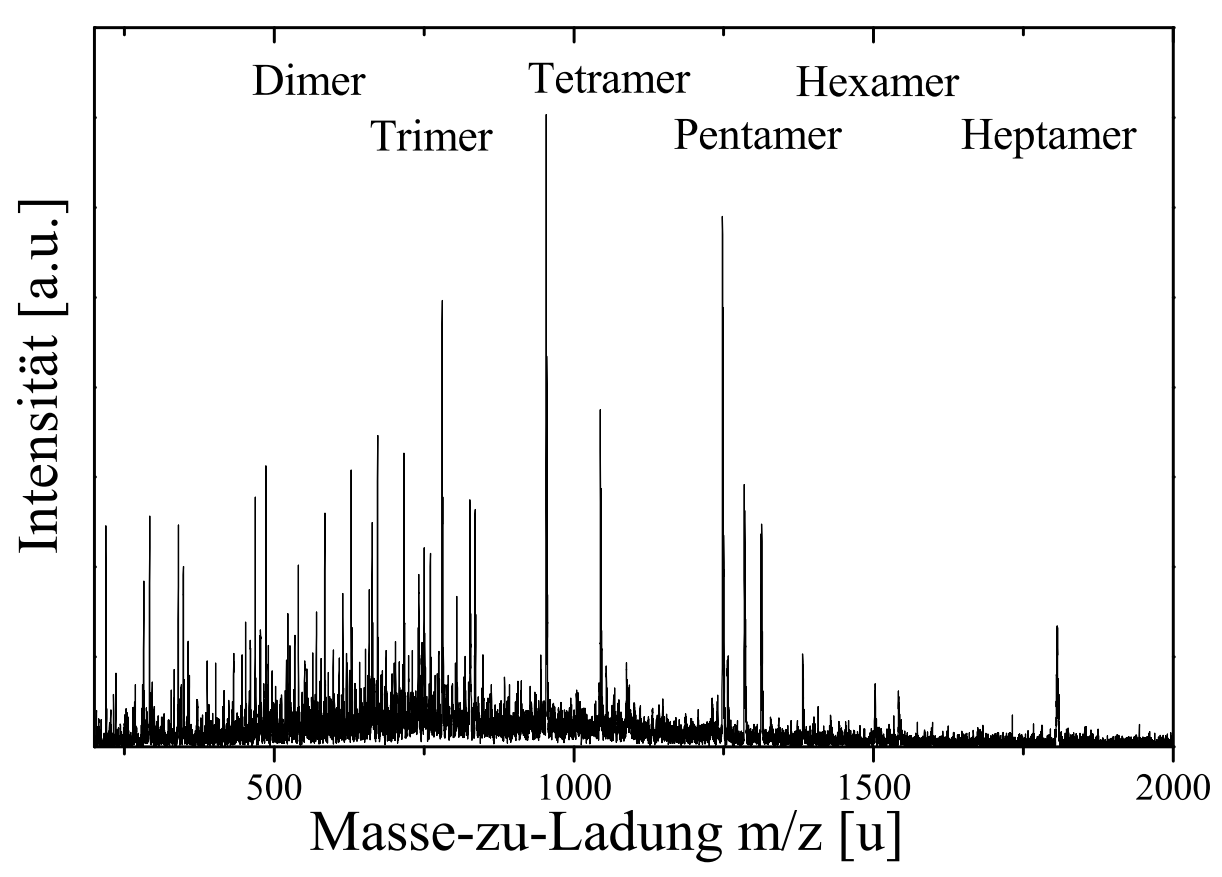

Abbildung 6.4: ESI Massenspektrum von thermisch aufgedampftem PC. Neben den höchsten Ausschlägen bei $m / z=954 \mathrm{u}, m / z=1248 \mathrm{u}$ und $m / z=780 \mathrm{u}$ treten noch viele weitere Signale vor allem im Bereich $450 \mathrm{u} \leq m / z \leq$ $750 \mathrm{u}$ auf. 


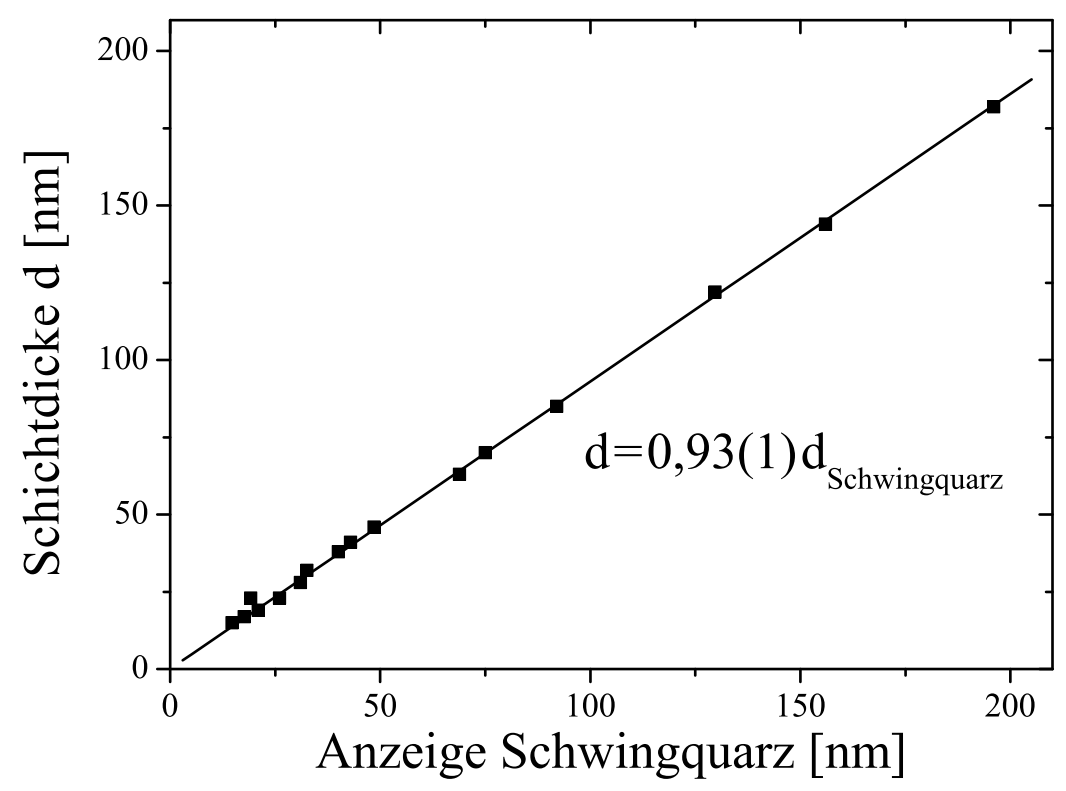

Abbildung 6.5: Die Schwingquarzwaage wird mit Hilfe von Kleinwinkelröntgenmessungen kalibriert. Man erkennt einen linearen Zusammenhang der Form $d=0,93(1) d_{\text {Schwingquarz }}$.

\subsection{Topographieentwicklung der thermisch aufgedampften PC Schichten}

\subsubsection{Bestimmung der Schichtdicke}

Zur Kontrolle der Schichtdicke steht in der Vakuumanlage eine Schwingquarzwaage zur Verfügung. Diese wird mit Hilfe von Kleinwinkelröntgenmessungen kalibriert, wie sie exemplarisch im Anhang in Abb. 11.2 gezeigt ist. Da die Schwingquarzwaage für metallische Schichten entwickelt ist und die Dichte des PC am unteren Ende der zugänglichen Dichte liegt ${ }^{2}$, ist nicht klar, dass man eine solche Waage verwenden kann. In Abb. 6.5 ist die durch SAXS gemessene Schichtdicke einiger Proben gegen die Anzeige des Schwingquarzes aufgetragen. Man erkennt einen linearen Zusammenhang der Form $d=0,93(1) d_{\text {Schwingquarz }}$, somit ist die Waage auch für die PC Schichten geeignet. Die Abweichung der Schwingquarzwaage beträgt etwa $7 \%$, wobei die angezeigten Schichtdicken systematisch etwas zu hoch liegen. Diese Abweichung resultiert zum einen aus der Geometrie, da der Schwingquarz nicht exakt an der gleichen Position wie das Substrat befestigt ist und zum anderen aus der sehr niedrigen Dichte des PCs, wegen der die Schwingquarzwaage im Grenzbereich der zugänglichen Dichte betrieben werden muss, wodurch ein größerer Fehler entstehen kann. Im Folgenden wird die Anzeige des Schwingquarzes als Maßs für die Dicke verwendet.

\footnotetext{
${ }^{2}$ Dieser Punkt betrifft die Einstellung des Materialparameters an der Schwingquarzwaage (max. 999, eingestellt 990).
} 


\subsubsection{Einfluss des Substrattyps auf die Topographie}

Um den Einfluss der Grenzfläche zum Substrat zu untersuchen, werden PC Schichten auf verschiedenen Substraten aufgedampft und mit dem AFM charakterisiert. Zunächst werden die anfänglichen Strukturen schichtdickenabhängig dargestellt. An dieser Stelle werden zur besseren Übersicht nur Topographien mit $4 \mu \mathrm{m}$ Kantenlänge vorgestellt. Für andere Bildgrößen $\left(1 \times 1 \mu \mathrm{m}^{2}, 2 \times 2 \mu \mathrm{m}^{2}\right.$ und $\left.6 \times 6 \mu \mathrm{m}^{2}\right)$ findet man ein qualitativ gleiches Verhalten, wobei die absoluten Zahlen aber um bis zu 20 \% abweichen können. Die hier vorgestellten Proben sind innerhalb von $24 \mathrm{~h}$ nach der Herstellung vermessen worden, da nicht alle Schichten über einen längeren Zeitraum stabil geblieben sind. Der Einfluss der Zeit folgt dann anschließend in Abs. 6.2.3.

\section{$\mathrm{Si}$}

Die Strukturentwicklung mit zunehmender Schichtdicke auf Si Substraten mit natürlicher Oxidschicht (vgl. Abs. 3.1.3) ist in Abb. 6.6 dargestellt. Während die Oberflächen bei dickeren Schichten $(d \gtrsim 40 \mathrm{~nm})$ homogen, glatt und ohne ausgezeichnete Struktur sind, weisen die dünneren Filme eine ausgeprägte Struktur mit grabenartigen Vertiefungen auf. Diese Veränderung in der Struktur äußert sich zudem in einem deutlichen Abfall der Rauigkeit mit zunehmender Schichtdicke, wie man es der Schichtdickenabhängigkeit der Rauigkeit in Abb. 6.7 für verschiedene Bildgrößen sehen kann. Die Rauigkeit sinkt von $\sigma(d=20 \mathrm{~nm})=7,9(3) \mathrm{nm}$ auf $\sigma(d \geq 50 \mathrm{~nm}) \leq 1,0(2) \mathrm{nm}$. In Abb. 6.8 sind einige exemplarische Höhen-Höhen-Korrelationsfunktionen (a), Höhendifferenzkorrelationsfunktionen (b) und Fourierspektren (c) für verschiedene Dicken gezeigt. Für dünnere Schichten weisen alle ein deutliches Maximum auf, aus dem die Korrelationslänge $R_{c}$, die einen mittleren Strukturabstand angibt, bestimmt werden kann. Mit zunehmender Schichtdicke steigt auch $R_{c}$ von etwa $180 \mathrm{~nm}$ bei $d=21 \mathrm{~nm}$ auf ca. $500 \mathrm{~nm}$ bei $d=170 \mathrm{~nm}$ an, allerdings werden die Maxima weniger ausgeprägt. Bei den aus den Höhendifferenzkorrelationsfunktionen berechneten Rauigkeitsexponenten zeigt sich, dass für dünne Schichten $\alpha \approx 0,85$ und für dicke Schichten $\alpha \approx 0,65$ gilt. Ebenso unterscheiden sich die Schichten im Abfall der Fourierspektren.

\section{$\mathrm{SiO}_{2}$}

Wie bei den Si Substraten ist auch die Strukturentwicklung mit zunehmender Schichtdicke auf $\mathrm{SiO}_{2}$, wie sie in Abb. 6.9 dargestellt ist, durch einen deutlichen Unterschied in der Topographie von dünnen und dickeren Schichten geprägt. Dickere Schichten mit $d \gtrsim 50 \mathrm{~nm}$ sind ähnlich zu den dickeren Schichten auf Si und auch sonst ist das Verhalten ähnlich zu den Ergebnissen auf Si. Bei den dünneren Schichten gibt es neben den grabenartigen Vertiefungen auch viele runde Öffnungen. In der Rauigkeitsentwicklung in Abb. 6.10 ist ein Abfall der Rauigkeit von $\sigma(d=20 \mathrm{~nm})=5,8(8) \mathrm{nm}$ auf $\sigma(d \geq 45 \mathrm{~nm}) \leq 1,0(3) \mathrm{nm}$ zu erkennen. In den Korrelationsfunktionen in Abb. 6.11 erkennt man wie beim Si klare Maxima bei den dünnen Schichten, die zu höheren Schichtdicken immer weniger ausgeprägt sind. Die Korrelationslänge $R_{c}$ steigt mit der Schichtdicke von etwa $230 \mathrm{~nm}$ bei $d=30 \mathrm{~nm}$ auf ca. $500 \mathrm{~nm}$ bei $d=100 \mathrm{~nm}$ an, der Rauigkeitexponent beträgt $\alpha \approx 0,9$ für dünne und $\alpha \approx 0,6$ für dickere Schichten. Der 
Abfall der Fourierspektren für dickere Schichten folgt $q^{-4}$, während er für die dünnsten Schichten (z. B. $d=30 \mathrm{~nm}$ ) steiler ist.

\section{GaAs}

Die Strukturentwicklung mit der Schichtdicke auf GaAs ist in Abb. 6.12 gezeigt. Hier sind die Strukturen deutlich ausgeprägter als auf $\mathrm{Si}$ und $\mathrm{SiO}_{2}$, auch wenn die Form gerade bei den dünneren Schichten ähnlich ist. Dies äußert sich auch in der Höhenskala, die mit $15 \mathrm{~nm}$ größer gewählt werden muss. Die Vertiefungen sind bei dünnen Schichten grabenartig und länglich, nehmen mit zunehmender Schichtdicke aber immer rundere Form an, außerdem nimmt ihre Tiefe ab. Diese Strukturen bleiben auch bei $d \geq 100 \mathrm{~nm}$ noch sichtbar. Dies wirkt sich auch auf den Rauigkeitsverlauf mit der Schichtdicke, der in Abb. 6.13 gezeigt ist, aus. Zunächst steigt die Rauigkeit stark an und hat bei $d \approx 25 \mathrm{~nm}$ mit $\sigma=14(1) \mathrm{nm}$ ihren maximalen Wert erreicht. Da die Tiefe der Struktur abnimmt, fällt auch die Rauigkeit mit der Schichtdicke ab. Bei $d \approx 170 \mathrm{~nm}$ findet man $\sigma \leq 0,8(1) \mathrm{nm}$. Dieser Wert ist vergleichbar mit den Restrauigkeiten auf Si und $\mathrm{SiO}_{2}$, dennoch unterscheiden sich die Topographien auf GaAs von denen auf Si und $\mathrm{SiO}_{2}$. In den Korrelationsfunktionen in Abb. 6.14 findet man für alle Schichtdicken deutliche Maxima. Die Korrelationslänge $R_{c}$ steigt mit zunehmender Schichtdicke von etwa $220 \mathrm{~nm}$ bei $d=20 \mathrm{~nm}$ auf ca. $500 \mathrm{~nm}$ bei $d=100 \mathrm{~nm}$, der Rauigkeitsexponent ist $\alpha \approx 0,9$ für dünne und $\alpha \approx 0,65$ für dickere Schichten. Der Abfall der Fourierspektren für dickere Schichten folgt etwa einem $q^{-4}$ Verlauf, während er für dünne Schichten $d \leq 45 \mathrm{~nm}$ steiler ist.

\section{PC}

Im Gegensatz zu den oben vorgestellten Schichten auf anorganischen Substraten ist für PC auf PC keine ausgezeichnete Struktur oder Schichtdickenabhängigkeit zu erkennen. Die Topographien für Schichten verschiedener Dicke, die in Abb. 6.15 gezeigt sind, ähneln sich und sind alle glatt und homogen, ohne Vertiefungen oder Risse. Die Rauigkeit schwankt für alle Dicken um den Wert $\sigma=0,5(2) \mathrm{nm}$ (vgl. Abb. 6.16). Aus den Korrelationsfunktionen in Abb. 6.17 kann die Korrelationslänge nur zu $R_{c} \approx 200 \mathrm{~nm}$ abgeschätzt werden, da die Maxima nur sehr schwach ausgeprägt, bzw. nicht vorhanden sind. Der Rauigkeitsexponent liegt zwischen $0,4 \leq \alpha \leq 0,65$ und der Abfall der Fourierspektren liegt zwischen $q^{-3}$ und $q^{-4}$. 

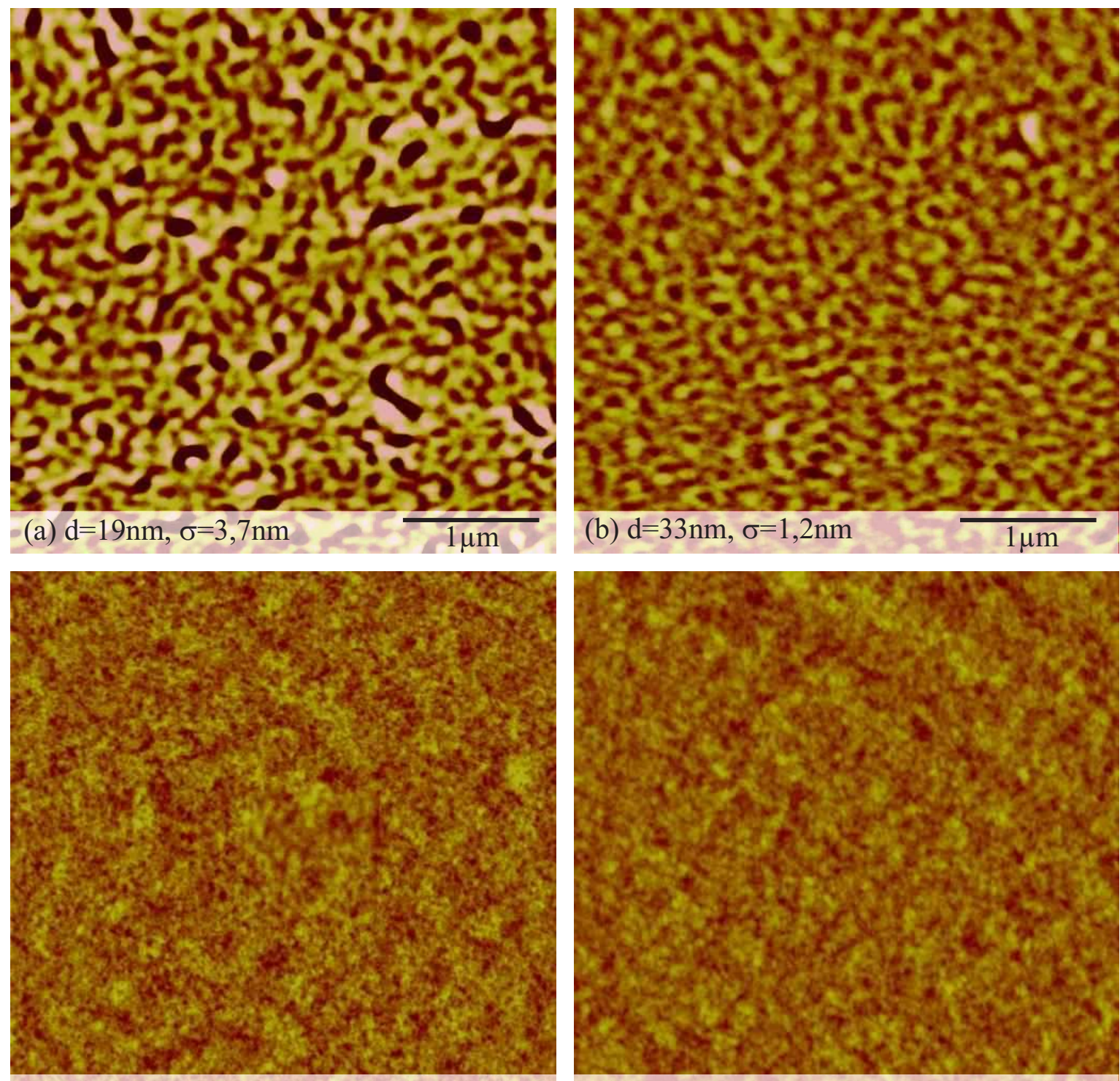

(c) $\mathrm{d}=90 \mathrm{~nm}, \sigma=0,7 \mathrm{~nm}$

$1 \mu \mathrm{m}$

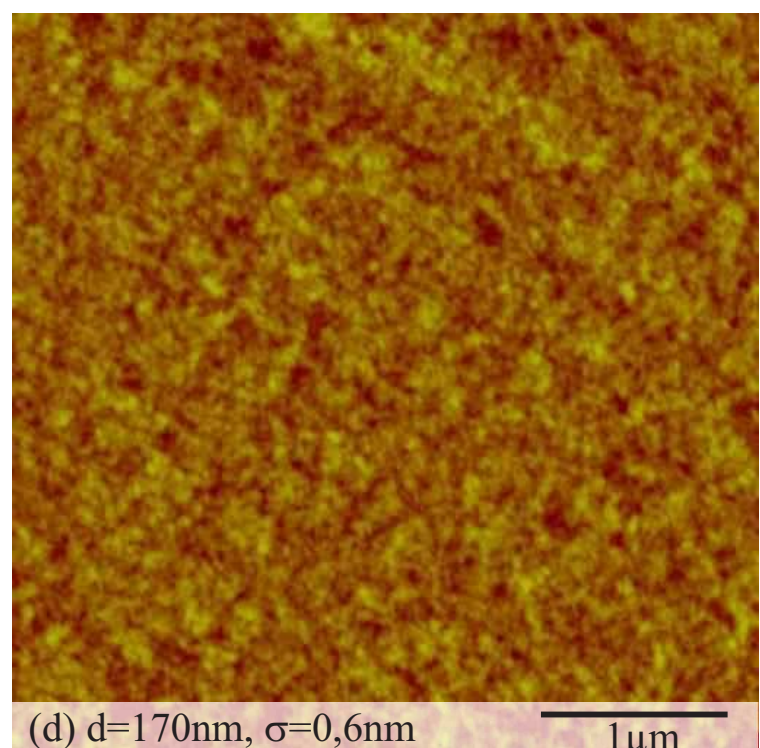

$0 \mathrm{~nm}$

$10 \mathrm{~nm}$

Abbildung 6.6: Topographie für verschiedene Schichtdicken von PC auf Si. Bei den dünnsten Schichten ist eine deutlich andere Struktur zu erkennen - statt einer homogenen, glatten Oberfläche wie bei den dickeren Schichten sind graben- und lochartige Strukturen zu sehen. 


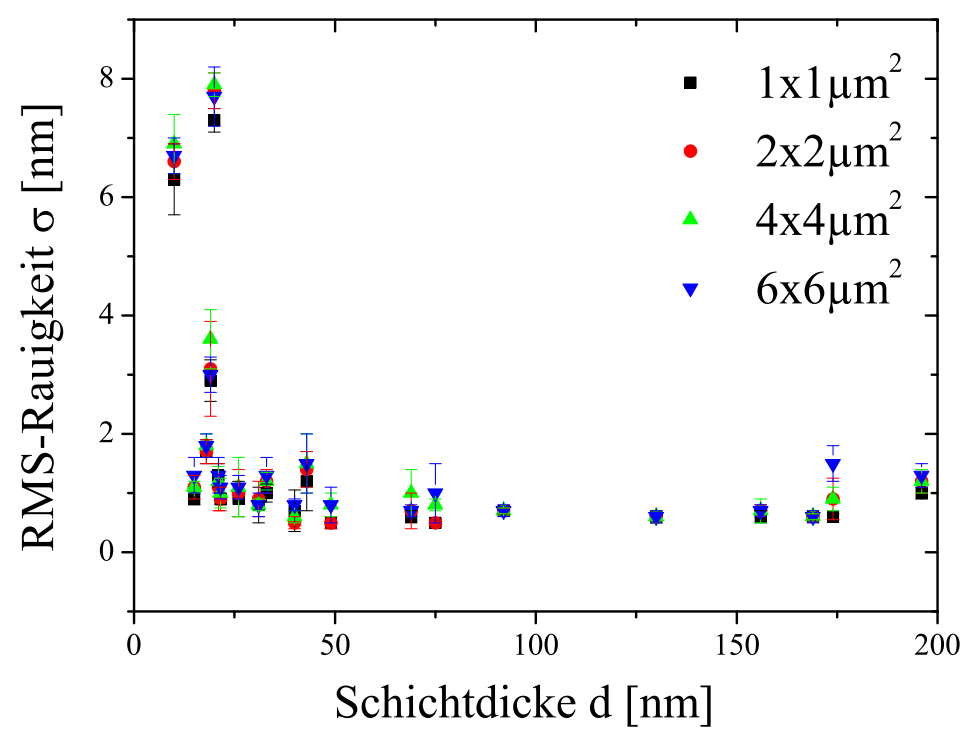

Abbildung 6.7: Rauigkeit in Abhängigkeit von der Schichtdicke auf Si für verschiedene Bildgrößen. Zu dünneren Schichten hin ist ein Anstieg in der Rauigkeit zu erkennen.
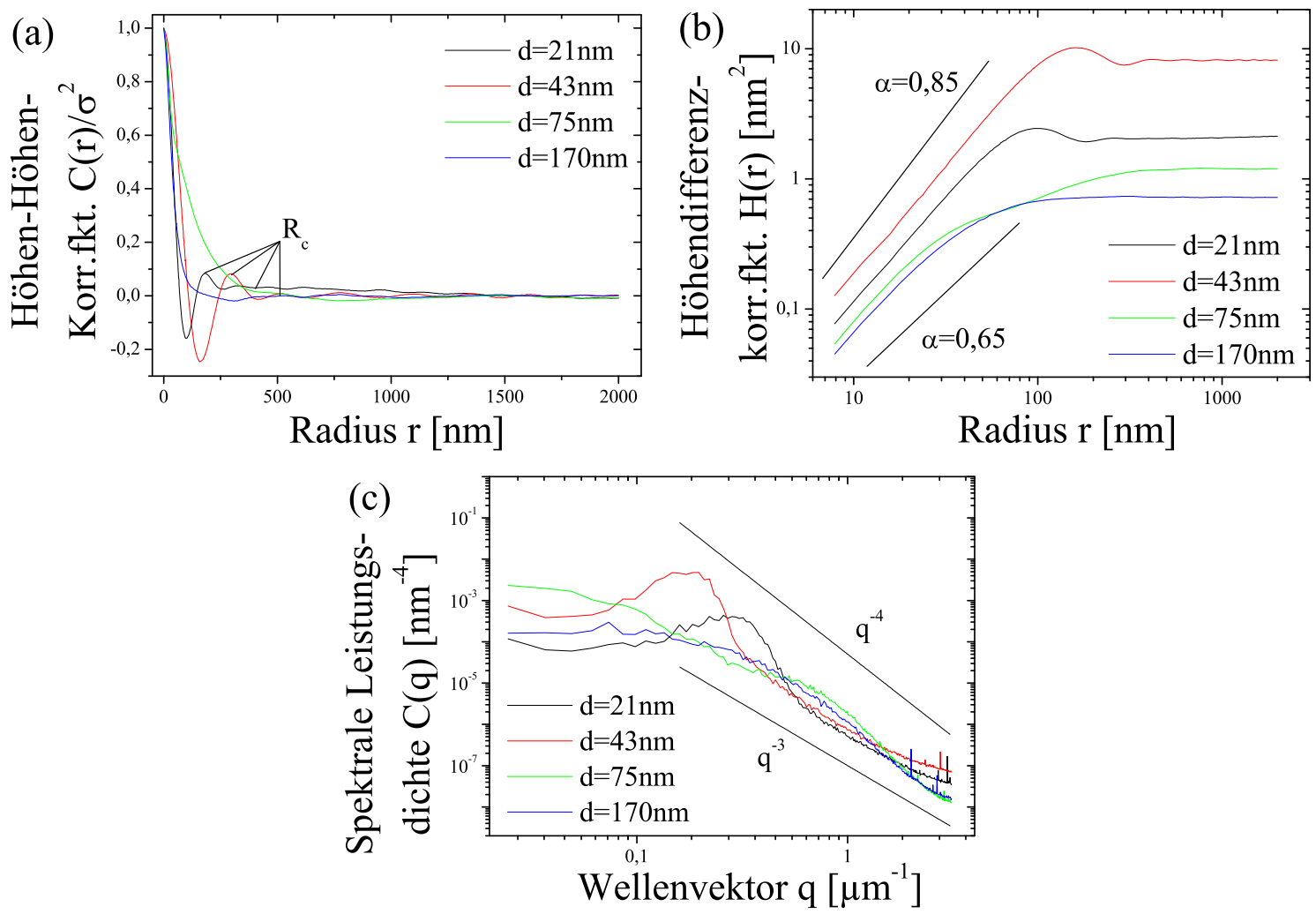

Abbildung 6.8: Korrelationsfunktionen für PC auf Si mit verschiedener Schichtdicke: (a) Höhen-Höhen-Korrelationsfunktion, (b) Höhendifferenzkorrelationsfunktion und (c) Fourierspektren. Mit zunehmender Schichtdicke wächst die Korrelationslänge $R_{c}$. 

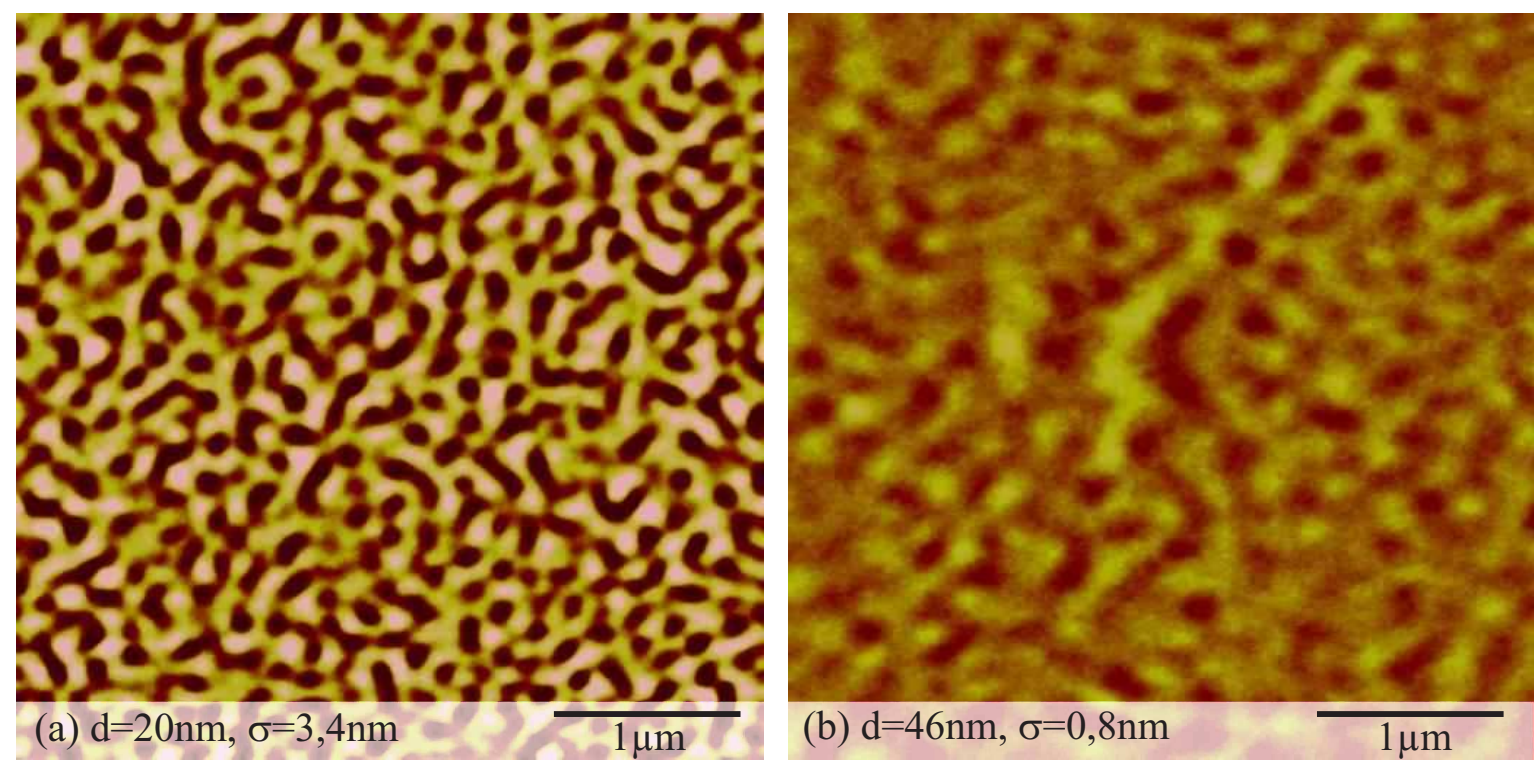

(b) $\mathrm{d}=46 \mathrm{~nm}, \sigma=0,8 \mathrm{~nm}$

$1 \mu \mathrm{m}$

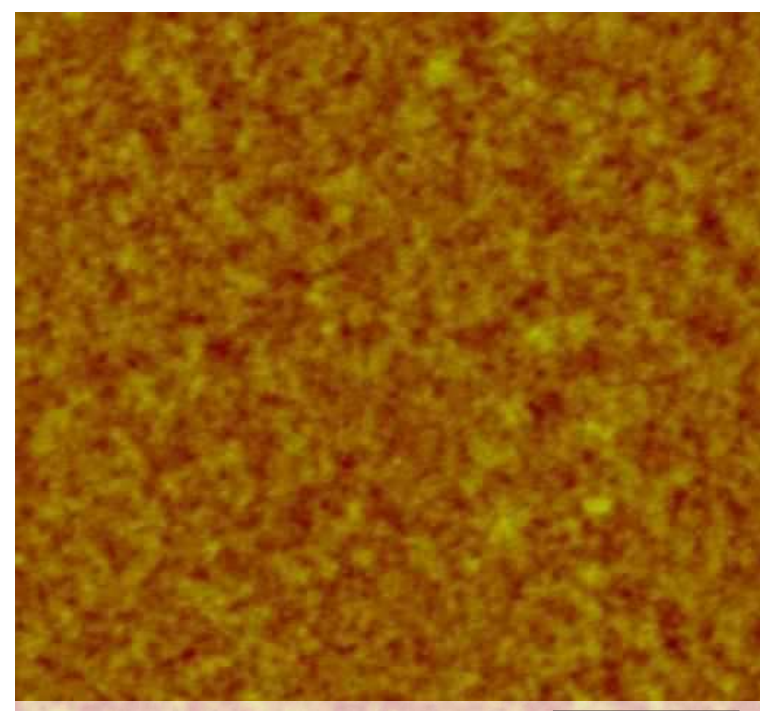

(c) $\mathrm{d}=70 \mathrm{~nm}, \sigma=0,4 \mathrm{~nm}$

$1 \mu \mathrm{m}$

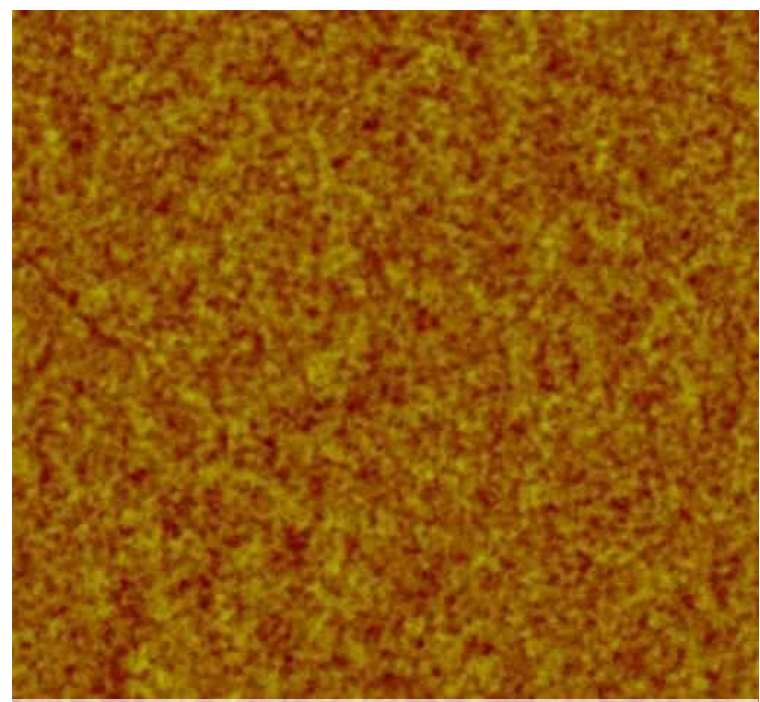

(d) $d=100 \mathrm{~nm}, \sigma=0,5 \mathrm{~nm}$

$1 \mu \mathrm{m}$

$0 \mathrm{~nm}$

$10 \mathrm{~nm}$

Abbildung 6.9: Topographie für verschiedene Schichtdicken von $\mathrm{PC}$ auf $\mathrm{SiO}_{2}$. Bei den dünnsten Schichten ist eine deutliche Strukturbildung zu erkennen, wohingegen die dickeren Schichten homogen und glatt sind. 


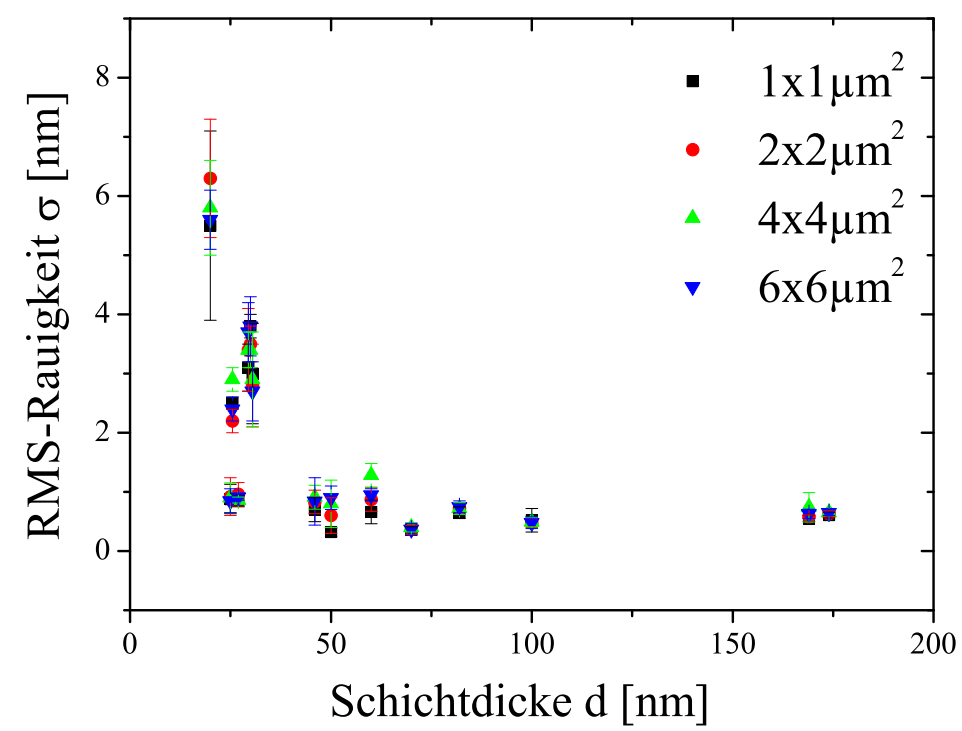

Abbildung 6.10: Rauigkeit in Abhängigkeit von der Schichtdicke auf $\mathrm{SiO}_{2}$ für verschiedene Bildgrößen. Zu dünneren Schichten hin ist ein Anstieg in der Rauigkeit zu erkennen.
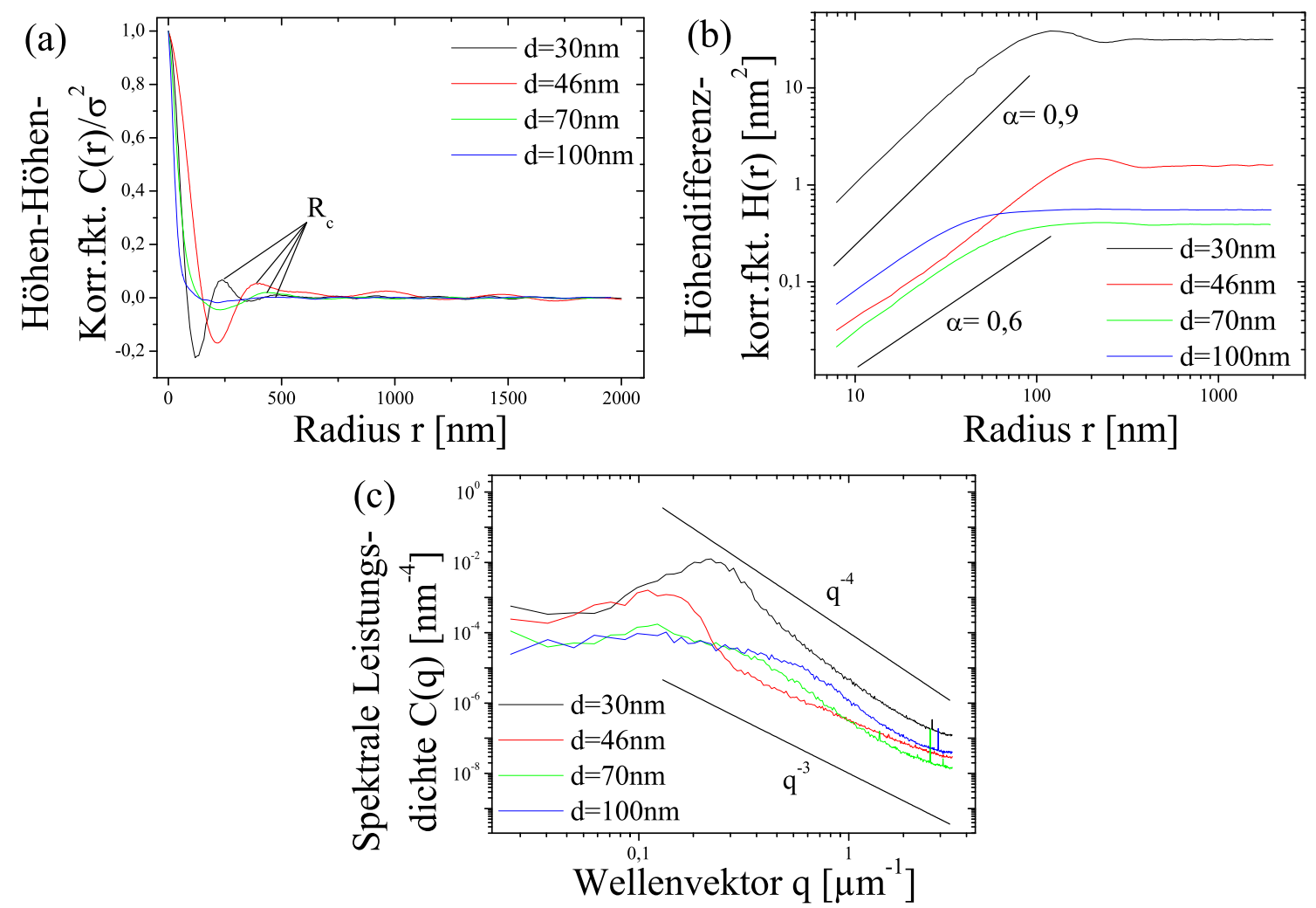

Abbildung 6.11: Korrelationsfunktionen für $\mathrm{PC}$ auf $\mathrm{SiO}_{2}$ mit verschiedener Schichtdicke: (a) Höhen-Höhen-Korrelationsfunktion, (b) Höhendifferenzkorrelationsfunktion und (c) Fourierspektren. Mit zunehmender Schichtdicke wächst die Korrelationslänge $R_{c}$. 

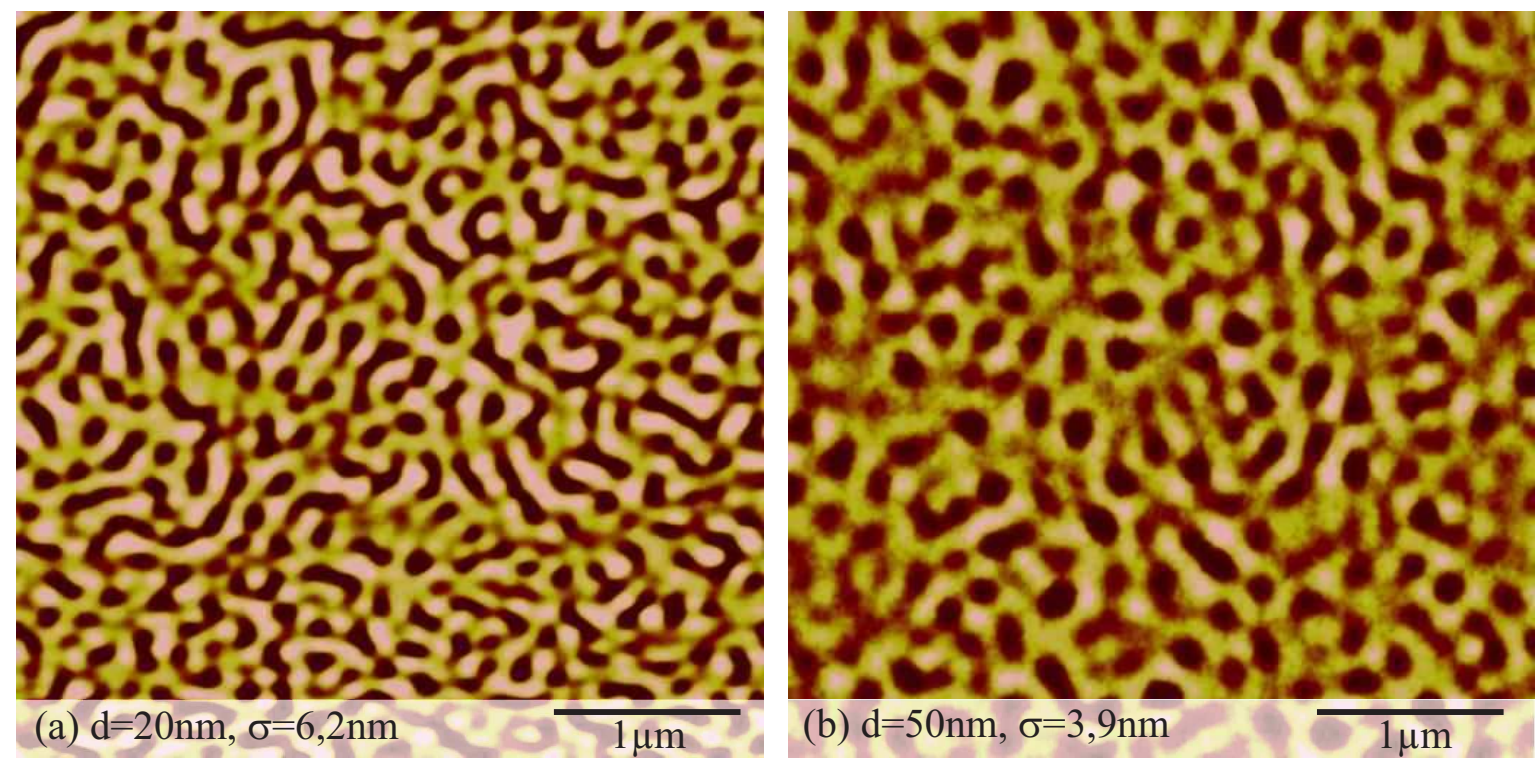

(b) $\mathrm{d}=50 \mathrm{~nm}, \sigma=3,9 \mathrm{~nm}$
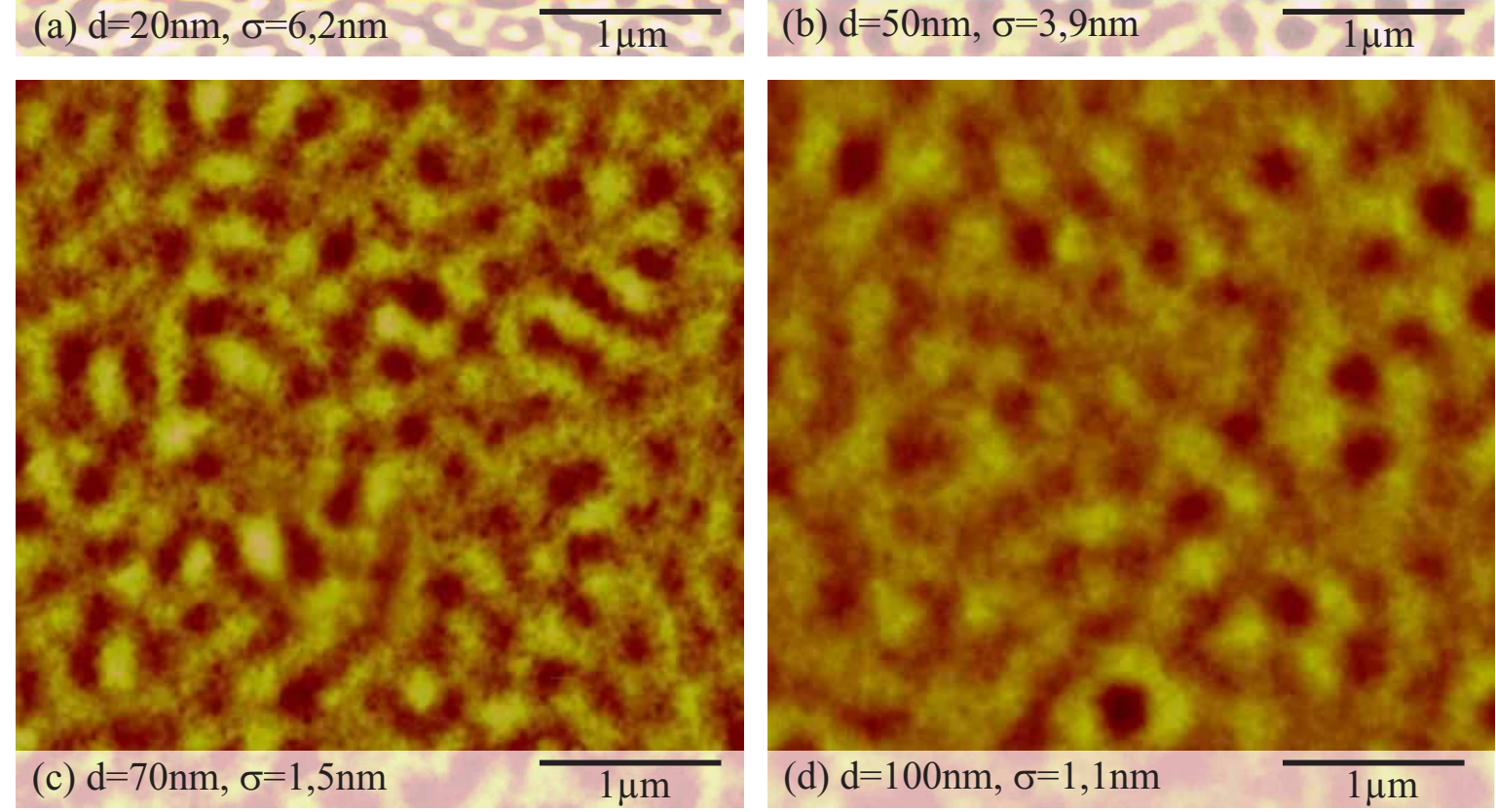

$0 \mathrm{~nm}$

$15 \mathrm{~nm}$

Abbildung 6.12: Topographie für verschiedene Schichtdicke von PC auf GaAs. Die Struktur ändert sich von grabenartigen Vertiefungen bei sehr dünnen Schichten zu eher runden Vertiefungen bei dickeren Schichten, deren Tiefe mit zunehmender Dicke abnimmt. 


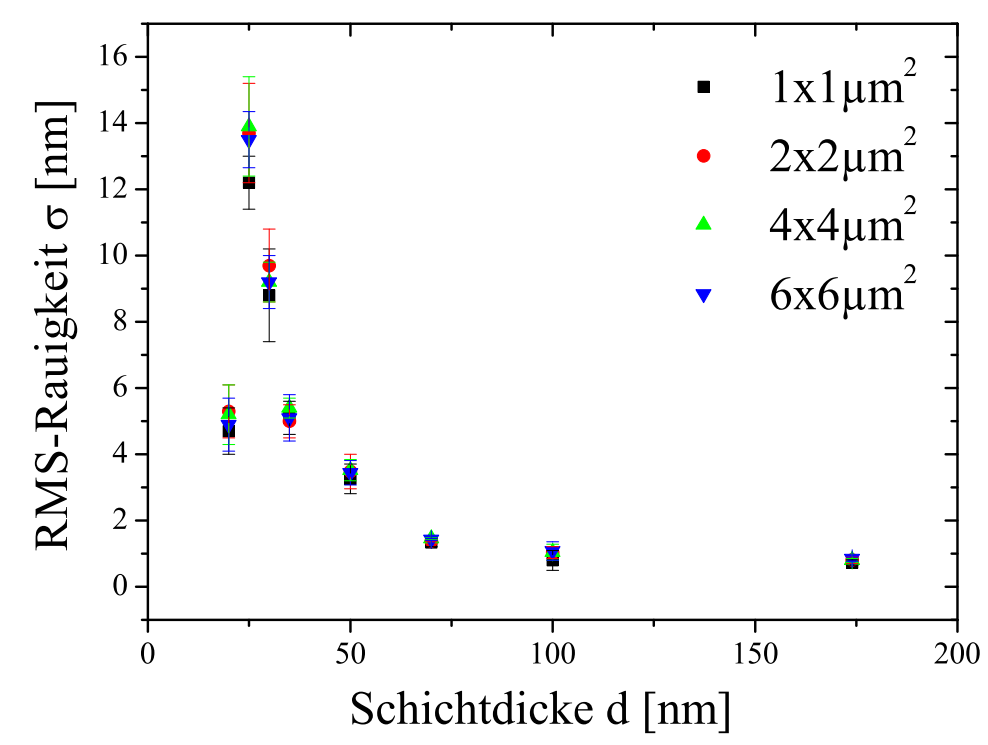

Abbildung 6.13: Rauigkeit in Abhängigkeit von der Schichtdicke auf GaAs für verschiedene Bildgrößen. Zunächst steigt die Rauigkeit stark an, um dann mit zunehmender Schichtdicke abzufallen.
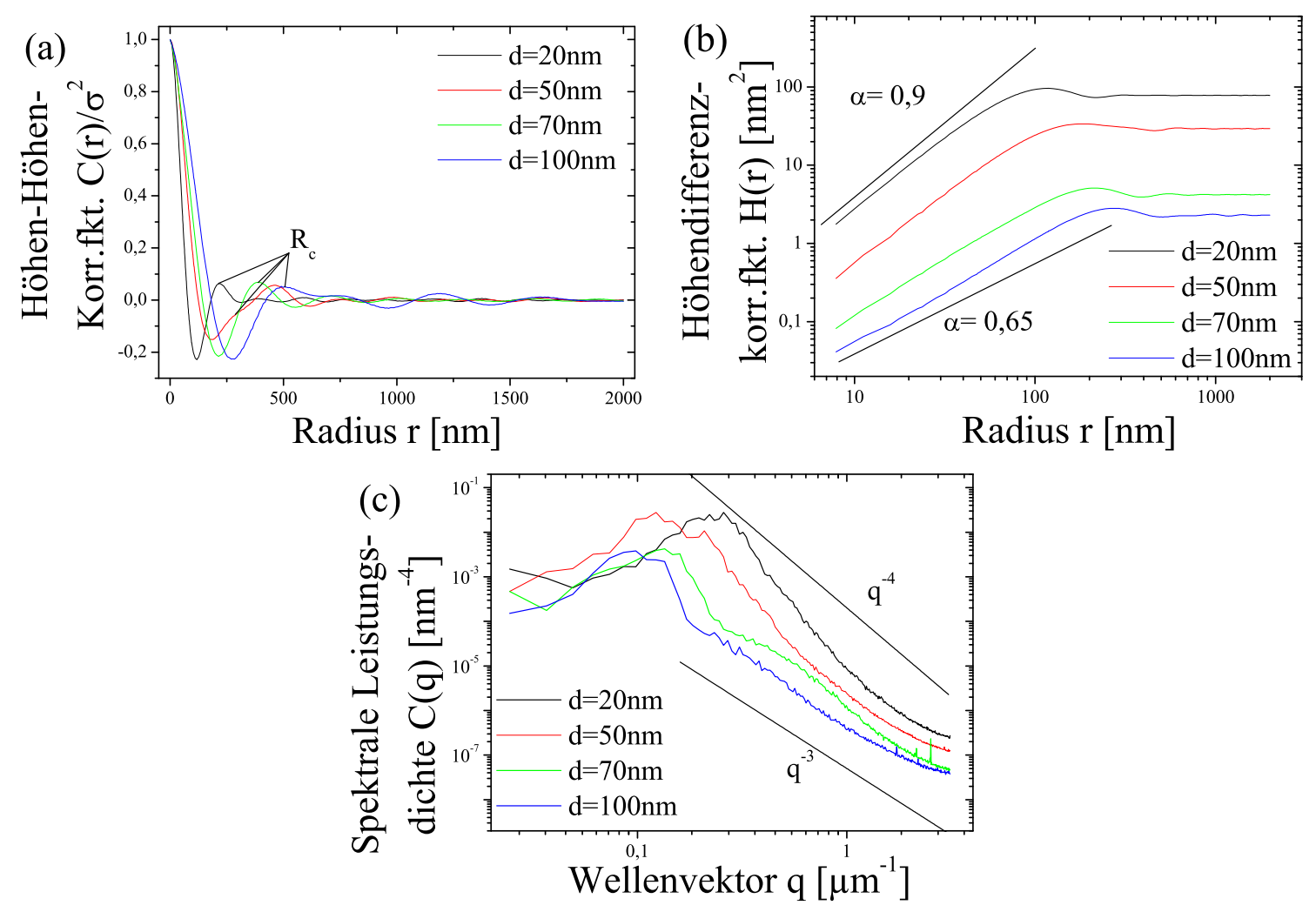

Abbildung 6.14: Korrelationsfunktionen für PC auf GaAs mit verschiedener Schichtdicke: (a) Höhen-Höhen-Korrelationsfunktion, (b) Höhendifferenzkorrelationsfunktion und (c) Fourierspektren. Mit zunehmender Schichtdicke wächst die Korrelationslänge $R_{c}$. 

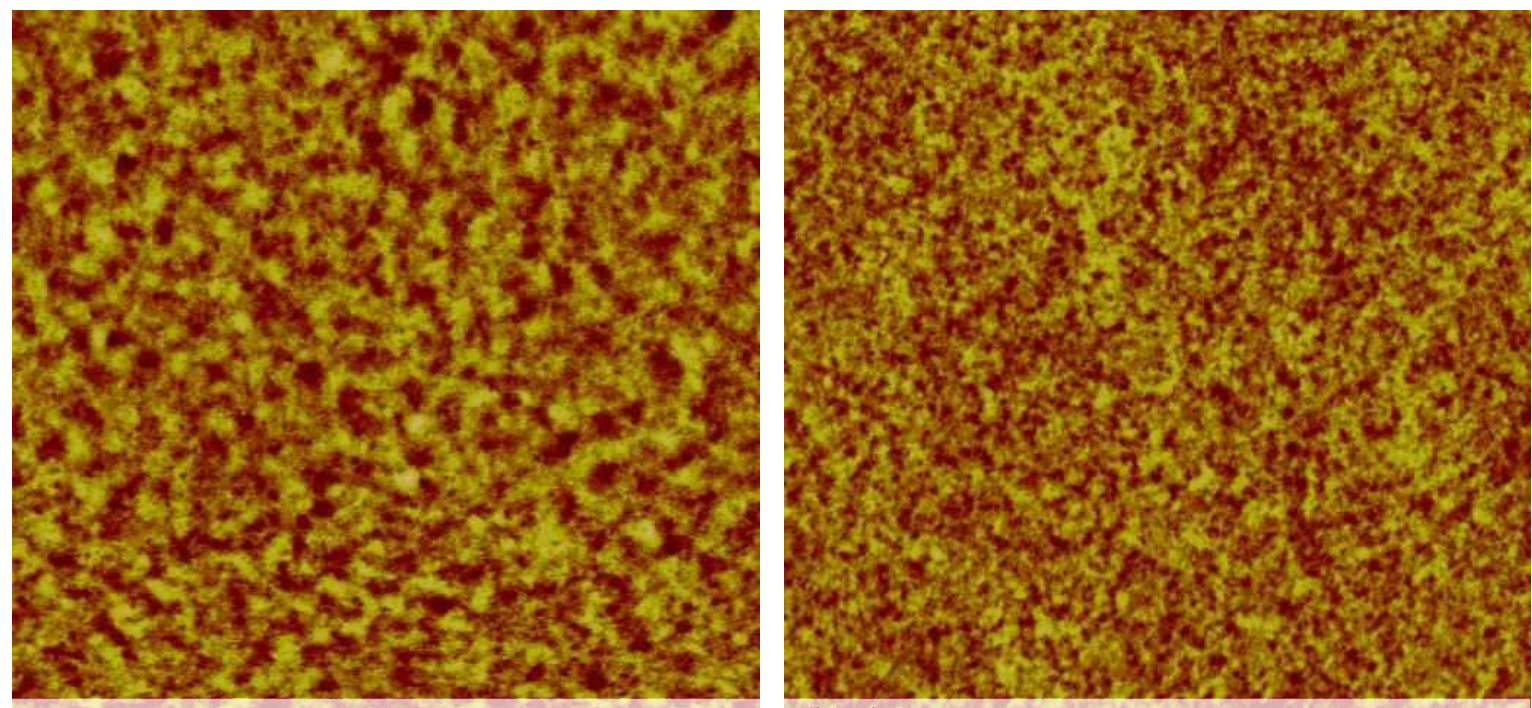

(a) $d=25 \mathrm{~nm}, \sigma=0,6 \mathrm{~nm}$

(b) $d=50 \mathrm{~nm}, \sigma=0,5 \mathrm{~nm}$

$1 \mu \mathrm{m}$

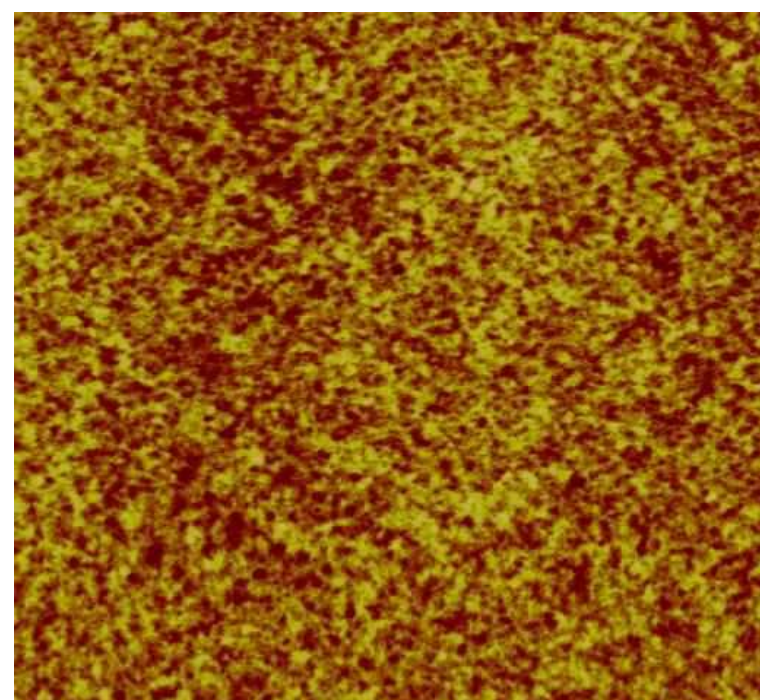

(c) $d=82 \mathrm{~nm}, \sigma=0,6 \mathrm{~nm}$

$1 \mu \mathrm{m}$

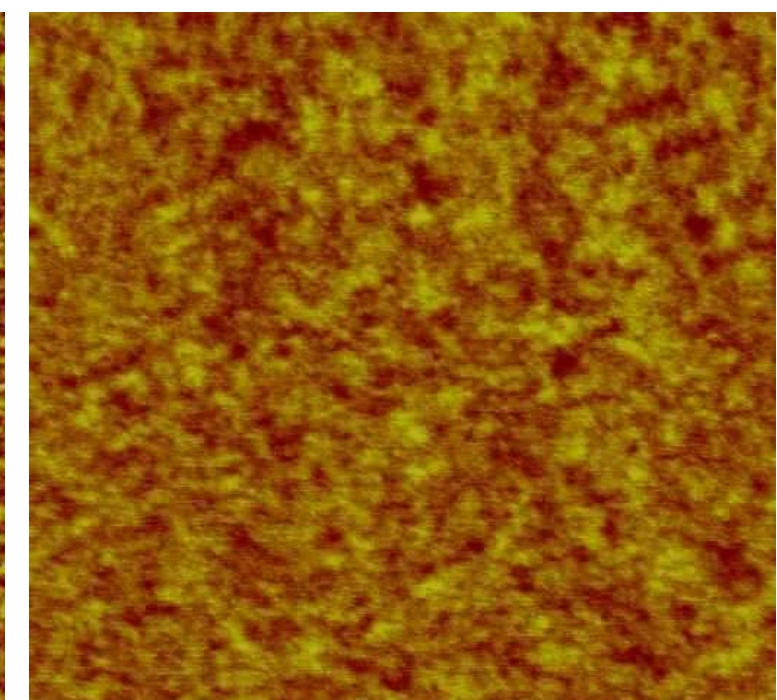

(d) $d=100 \mathrm{~nm}, \sigma=0,4 \mathrm{~nm}$

$1 \mu \mathrm{m}$

$0 \mathrm{~nm}$ $5 \mathrm{~nm}$

Abbildung 6.15: Topographie für verschiedene Schichtdicken von PC auf PC. Die Schichten sind für alle Dicken ähnlich glatt. 


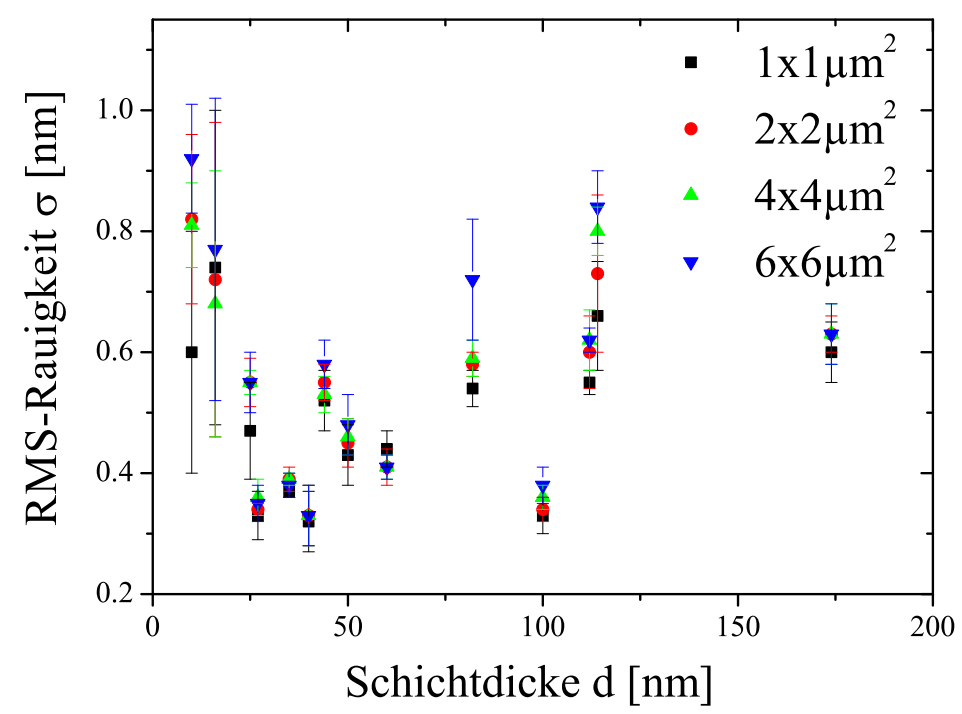

Abbildung 6.16: Rauigkeit in Abhängigkeit von der Schichtdicke auf PC für verschiedene Bildgrößen. Alle Werte schwanken statistisch um $\sigma=0,5(2) \mathrm{nm}$, ohne eine systematische Abhängigkeit von der Schichtdicke zu zeigen.
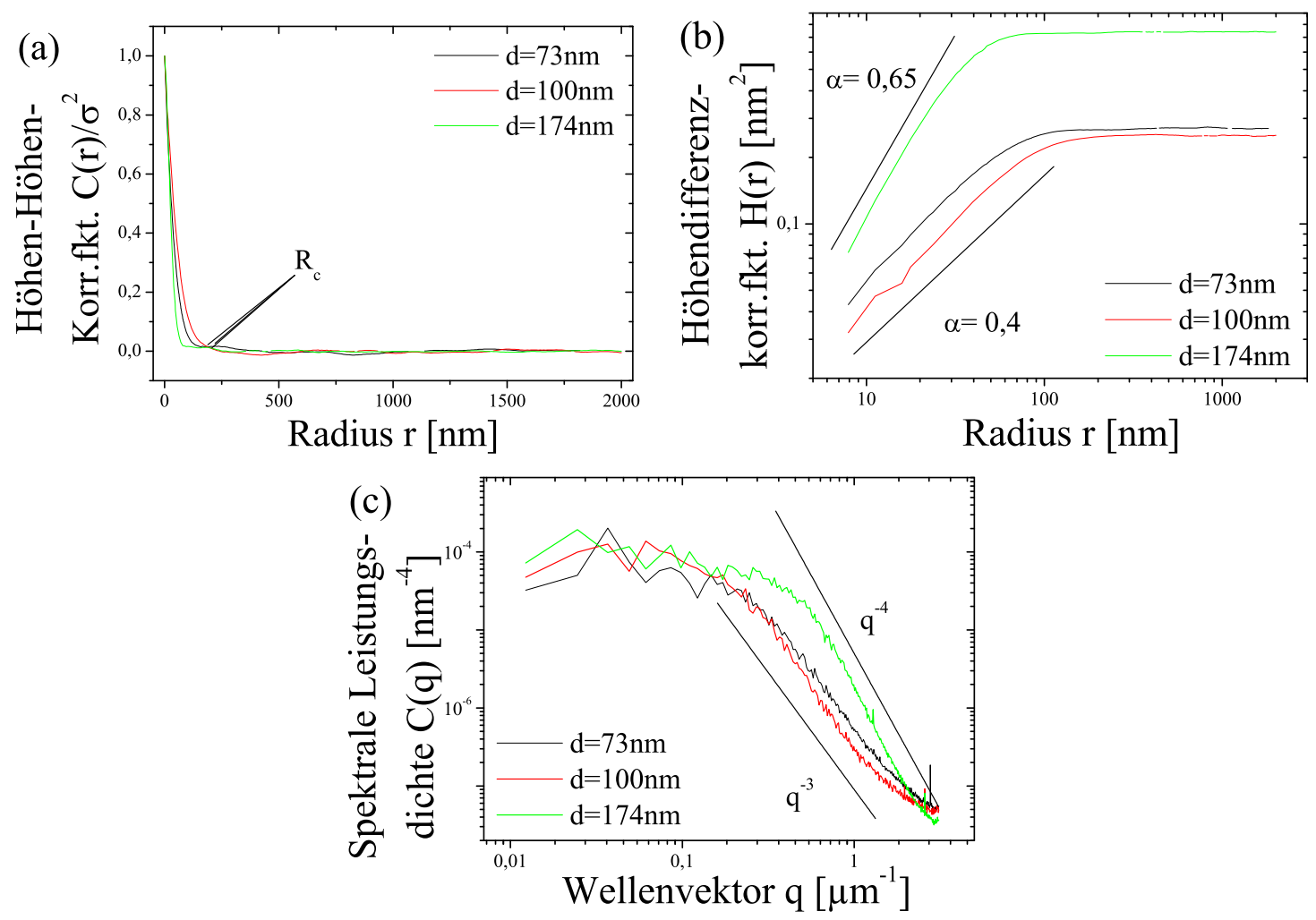

Abbildung 6.17: Korrelationsfunktionen für PC auf PC mit verschiedener Schichtdicke: (a) Höhen-Höhen-Korrelationsfunktion, (b) Höhendifferenzkorrelationsfunktion und (c) Fourierspektren. Die Maxima zur Bestimmung der Korrelationslänge $R_{c}$ sind nur schwach ausgeprägt, bzw. nicht vorhanden. 

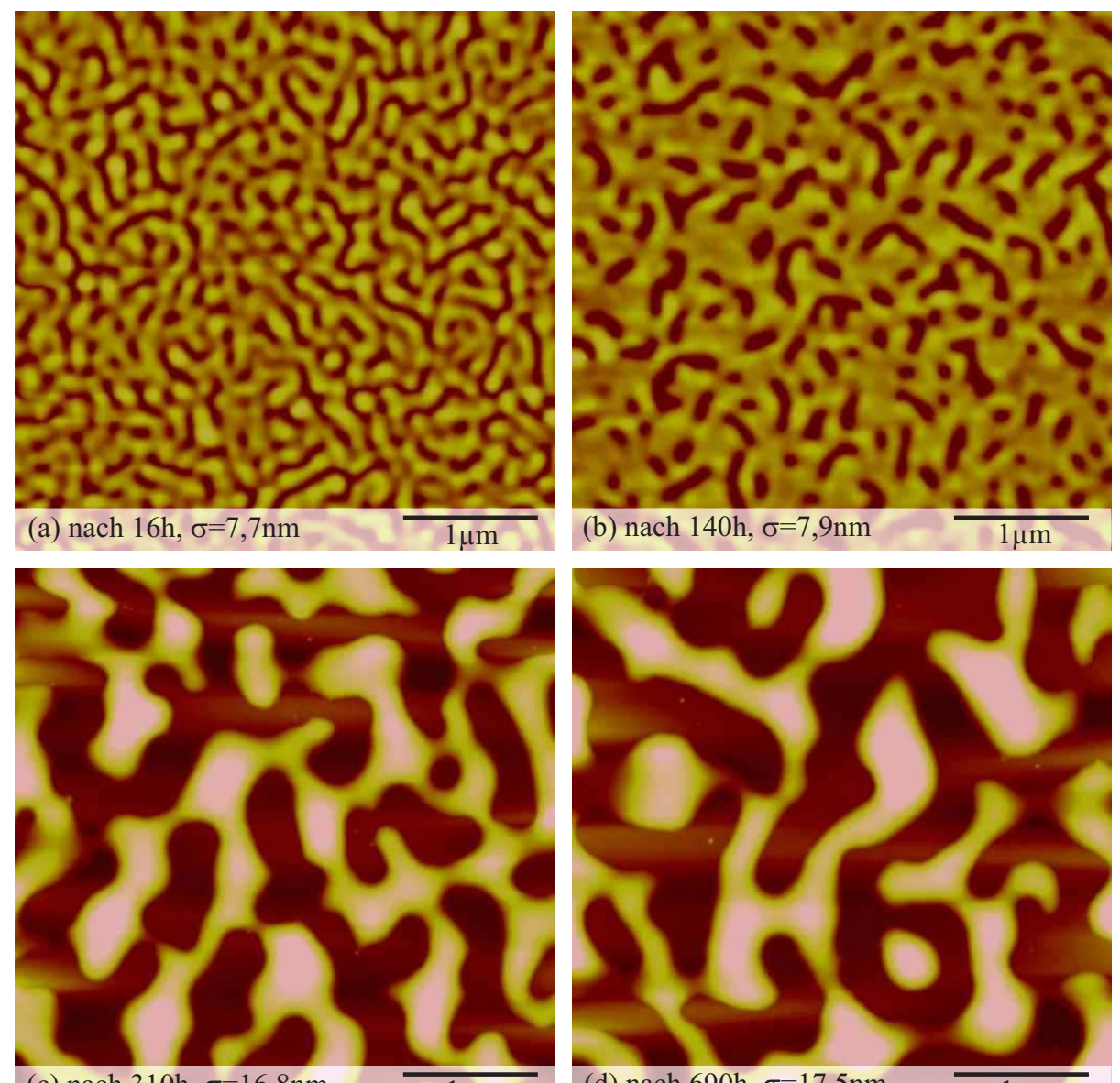

(c) nach $310 \mathrm{~h}, \sigma=16,8 \mathrm{~nm}$

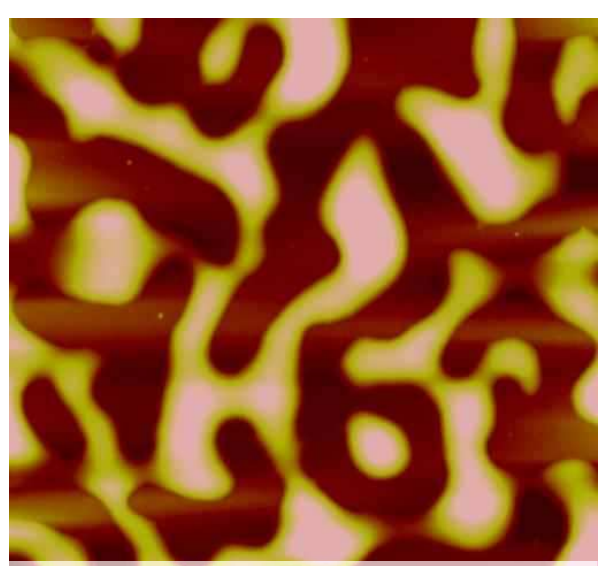

(d) nach $690 \mathrm{~h}, \sigma=17,5 \mathrm{~nm}$

$1 \mu \mathrm{m}$

$0 \mathrm{~nm}$

$60 \mathrm{~nm}$

Abbildung 6.18: Topographie einer $20 \mathrm{~nm}$ dicken PC Schicht auf Si gemessen (a) $16 \mathrm{~h}$, (b) $140 \mathrm{~h}$, (c) $310 \mathrm{~h}$ und (d) $690 \mathrm{~h}$ nach der Herstellung. Mit zunehmender Lagerungszeit bilden sich größere inselartige Strukturen.

\subsubsection{Zeitliche Entwicklung der Topographie}

Neben der Strukturbildung während der Herstellung und den damit verbundenen anfänglichen Strukturen zeigt sich bei den dünnen Schichten eine langsame Änderung der Struktur über einen Zeitraum von mehreren Tagen, bzw. Wochen. Die dickeren Schichten mit Dicken $d \gtrsim 40 \mathrm{~nm}$ zeigen dagegen auch nach Monaten keine deutliche Veränderung.

Abb. 6.18 zeigt diese zeitliche Entwicklung anhand einer $20 \mathrm{~nm}$ dicken PC Schicht auf $\mathrm{Si}$, die zu verschiedenen Zeiten nach der Herstellung vermessen worden ist. Die Lagerung der Proben erfolgt an Luft bei Raumtemperatur. Mit zunehmender Lagerungszeit bilden sich immer höhere Inseln und das Material reduziert die Kontaktfläche zum Substrat. Dies ist ebenfalls in Abb. 6.19 am Anteil der unbedeckten Fläche zu sehen. Dieser steigt von anfangs etwa $10 \%$ bis zu 50 \% innerhalb von 690 h an. Es bilden sich höhere inselartige Strukturen, die untereinander nur noch durch schmale Hälse verbunden sind. Mit zunehmender Zeit verschwinden die Hälse und es bleiben isolierte Inseln zurück. 


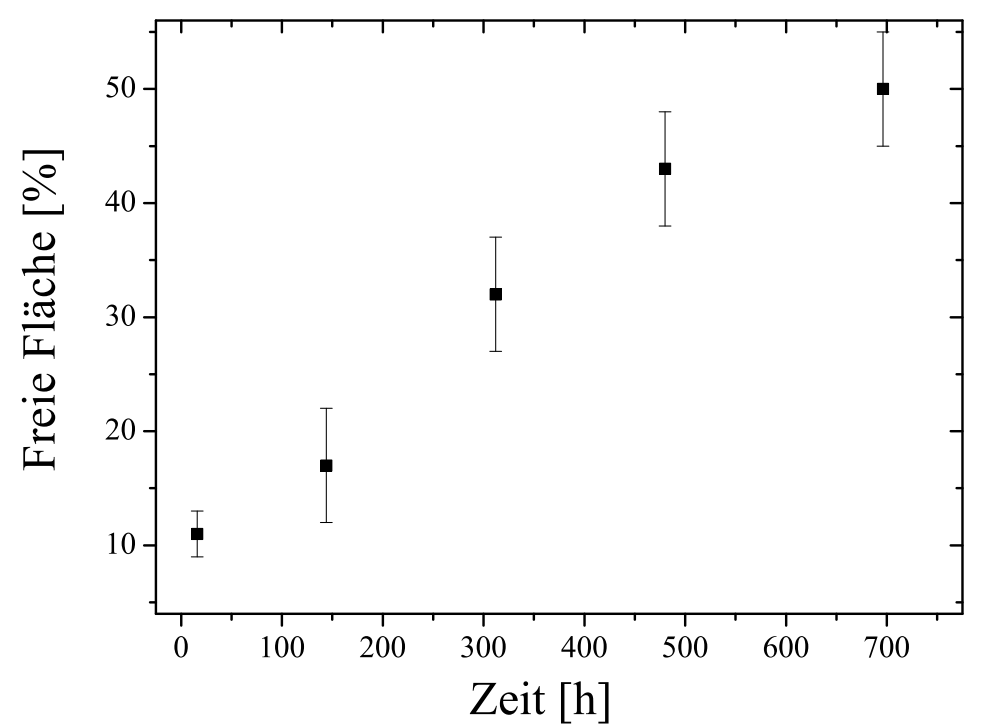

Abbildung 6.19: Anteil der unbedeckten Fläche einer $20 \mathrm{~nm}$ dicken PC Schicht auf Si in Abhängigkeit von der Lagerungszeit. Mit zunehmender Lagerungszeit wird immer mehr Fläche des Substrats freigelegt.

\subsection{Lokale mechanische Eigenschaften}

\subsubsection{Messungen mit Kraft-Abstandskurven}

Um die lokalen Unterschiede von mechanischen Eigenschaften zu untersuchen, werden wie in Abs. 3.2.5 beschrieben Kraft-Abstandskurven, bzw. Kraft-Verschiebungskurven, an verschiedenen Stellen einer Probe aufgenommen, um eine Karte dieser Eigenschaften zu erstellen. Den hier vorgestellten Messungen liegt ein Raster von $65 \times 63$ Punkten $^{3}$ mit einem Abstand von jeweils $30 \mathrm{~nm}$ zugrunde, das mit einer Rate von einer Kraft-Verschiebungskurve pro Sekunde gemessen wird. Um die Messungen untereinander vergleichen zu können, ist das sog. FIEL-Mapping (vgl. Abs. 3.2.5) verwendet worden. Bei jeder Kraft-Verschiebungskurve ist die maximale Auslenkung des Cantilevers auf $5 \mathrm{~nm}$ begrenzt und alle Kurven werden mit demselben Cantilever gemessen. Aufgetragen werden die (normierten) Flächen unter der Annäherungslinie der KraftAbstandskurven. In Fig. 6.20 sind zum Vergleich Karten sowohl von thermisch aufgedampftem $\mathrm{PC}$ auf $\mathrm{Si}, \mathrm{SiO}_{2}$ und $\mathrm{PC}$ als auch von $\mathrm{PC}$ und $\mathrm{SiO}_{2}$ Substraten gezeigt (vgl. Abs. 3.1.3). Die thermisch aufgedampften Schichten sind alle zur gleichen Zeit in einem Verdampfungsvorgang hergestellt worden und sind ca. $70 \mathrm{~nm}$ dick. Alle Oberflächen sind mit RMS-Rauigkeiten $\sigma \leq 0,5 \mathrm{~nm}$ so glatt, dass die Topographien keinen Einfluss auf die Messungen haben sollte. Die gemessenen Werte liegen dabei gaußverteilt um den Mittelwert 1 (wegen der Normierung). Die Schwankungen, also die Halbwertsbreiten der angepassten Gaußverteilung, sind auf dem leeren PC Substrat am größten $(0,4)$ und auf dem $\mathrm{SiO}_{2}$ am geringsten (0,08). Für die thermisch aufgedampften Schichten findet man Halbwertsbreiten von 0,12 auf $\mathrm{Si}, 0,11$ auf $\mathrm{SiO}_{2}$ und 0,15 auf PC. Auf dem PC erkennt

\footnotetext{
${ }^{3}$ Zur Korrektur von Drift nach größeren Stellbewegungen des Piezos werden die ersten beiden Spalten nicht ausgewertet, so dass das effektive Raster aus $63 \times 63$ Punkten besteht.
} 
man größere Bereiche mit ähnlichen Eigenschaften. Bei den thermisch aufgedampften PC Schichten ist kein weiterer Unterschied zwischen den verschiedenen Substraten zu erkennen. Die Bereiche mit ähnlichen Eigenschaften sind klein und umfassen selten mehr als ca. $100 \mathrm{~nm}$. Aus den Steigungen $m$ der Kraft-Abstandskurven im Kontaktbereich kann man Rückschlüsse auf die Härte der Proben ziehen. Diese sind für die thermisch aufgedampften PC Schichten mit $m \approx 2,1(3) \mathrm{N} / \mathrm{m}$ auf Si und $m \approx 2,0(3) \mathrm{N} / \mathrm{m}$ auf $\mathrm{SiO}_{2}$ und $\mathrm{PC}$ etwa gleich. Das PC Substrat ist etwas härter $(m \approx 2,3(2) \mathrm{N} / \mathrm{m})$ und das $\mathrm{SiO}_{2}$ Substrat mit $m \approx 3,1(2) \mathrm{N} / \mathrm{m}$ am härtesten.

Bei der Interpretation dieser Messungen muss allerdings beachtet werden, dass die verwendeten Methoden streng genommen nur im elastischen Bereich gelten und wie bereits in Abs. 3.2.5 angemerkt, ist plastische Verformung der weichen Polymeroberflächen stets ein Problem. Dies ist in Abb. 6.21(a)-(d) verdeutlicht, wo die Topographien vor und nach den Indentationsexperimenten gezeigt sind. Auf dem $\mathrm{SiO}_{2}$ Substrat (Abb. 6.21(e)) sind keine Veränderungen zu erkennen, während alle PC Schichten (aufgedampft und Substrat) plastisch verformt sind. Mit Höhenunterschieden unter $3 \mathrm{~nm}$ sind diese Veränderungen klein, beeinflussen aber die Ergebnisse. Stärker fällt die Verformung aus, wenn die maximale Auslenkung nicht oder weniger beschränkt, bzw. für die Messung ein Cantilever mit einer Federkonstante von $40 \mathrm{~N} / \mathrm{m}$ verwendet wird. Dafür ist ein Beispiel in Abb. 6.21(f) gezeigt, bei dem nach der Messung Höhenunterschiede von $20 \mathrm{~nm}$ und mehr auftreten. In Abb. 6.21(g) sind zudem zwei typische Kraft-Verschiebungskurven gezeigt, eine auf $\mathrm{SiO}_{2}$ und eine auf thermisch verdampftem $\mathrm{PC}$ auf $\mathrm{SiO}_{2}$. Die Hysterese zwischen Hin- und Rückweg auf der PC Probe ist ein Anzeichen für plastische Verformung, das auf $\mathrm{SiO}_{2}$ nicht zu sehen ist.

\subsubsection{Messungen mit Ultraschall-Kraftmikroskopie}

Um die Funktion des neuen AFAM Aufbaus zu demonstrieren, sind in Abb. 6.22 zwei Beispiele für den Bild-Modus des AFAMs (vgl. Abs. 3.2.5) zu sehen. Zum einen ist ein $500 \times 500 \mathrm{~nm}^{2}$ großer Bildausschnitt von einer Messung mit $2550 \mathrm{kHz}$ Anregungsfrequenz an Nickel gezeigt (vgl. Abb. 3.6) und zum anderen $2 \times 2 \mu^{2}$ mit $1350 \mathrm{kHz}$ Anregungsfrequenz an Eisen-Palladium. Links ist jeweils die Topographie - gemessen im Kontaktmodus - zu sehen und rechts die dazugehörigen AFAM Amplituden. Die AFAM Bilder beinhalten qualitative Informationen über die Kontaktsteifigkeit, so dass hier härtere Bereiche heller sind (beide Anregungsfrequenzen liegen jeweils etwas unterhalb der zugehörigen Kontaktresonanzfrequenz). Während in den Topographie-Bildern die Struktur der Oberfläche durch die großen Höhenunterschiede schwer erkennbar ist, sind die einzelnen Körner der polykristallinen Schichten im Amplitudenbild gut zu sehen. Die unterschiedlichen Kontaktsteifigkeiten verschiedener Körner sind ein Indiz für unterschiedliche Orientierungen.

Beispiele für Messungen im Spektroskopie-Modus sind in Abb. 6.23 anhand von Messungen auf Kalibrierungsproben gezeigt. Dafür sind mit demselben Cantilever bei gleicher Auflagekraft ${ }^{4}$ des Cantilevers und Anregungsstärke des Ultraschall-Prüfkopfs an jeweils 100 benachbarten Positionen $\left(10 \times 10\right.$ Punkte Raster) auf $\mathrm{SiO}_{2}$, Strontiumtitanat(100) und Saphir(101) Resonanzkurven aufgenommen und die Resonanzfrequen-

\footnotetext{
${ }^{4}$ Die Auflagekraft wird durch die Auslenkung des Cantilevers eingestellt.
} 

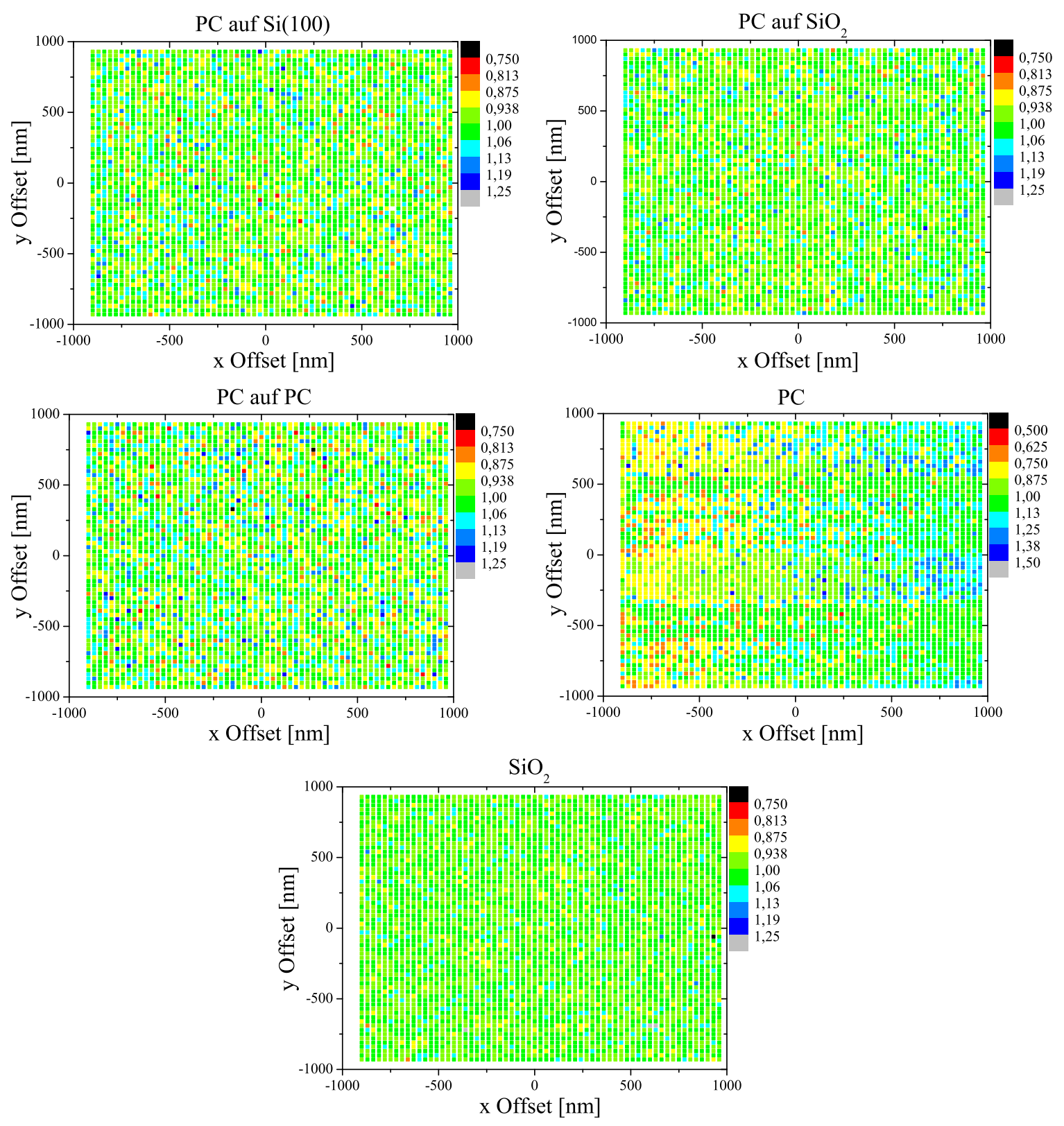

Abbildung 6.20: Karte der lokalen elastischen Eigenschaften von thermisch aufgedampftem $\mathrm{PC}$ auf verschiedenen Substraten im Vergleich mit einem $\mathrm{SiO}_{2}$ und einem PC Substrat (vgl. Abs. 3.1.3). Die aus den Flächen unter den Annäherungslinien der Kraft-Abstandskurven berechneten Werte sind wie in Abs. 3.2.5 beschrieben normiert (und daher einheitenlos). Die Werte liegen gaußverteilt um den Mittelwert 1. Es ist zu erkennen, dass die Schwankungen des $\mathrm{SiO}_{2}$ Substrats am geringsten sind. 

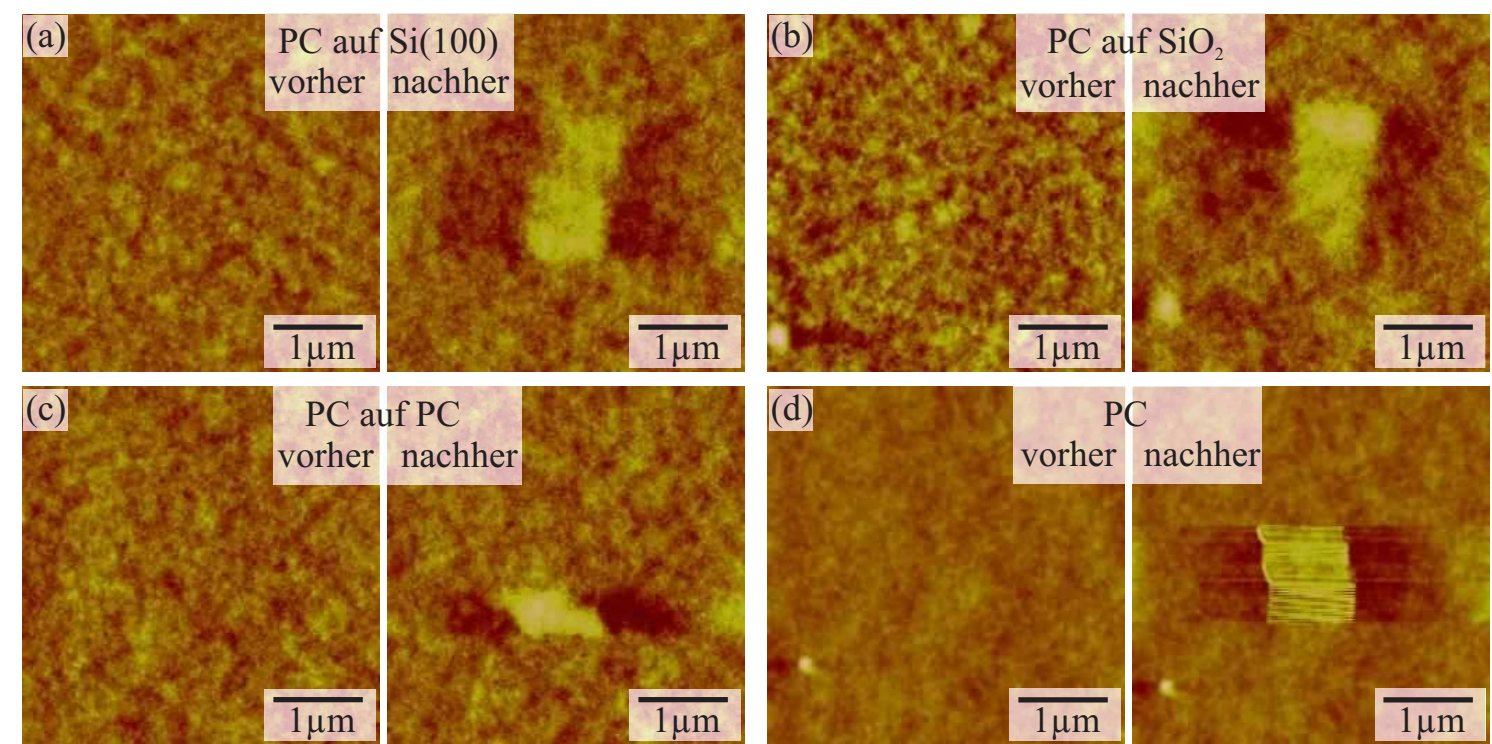

$\mathrm{PC}$ auf $\mathrm{PC}$

vorher nachher
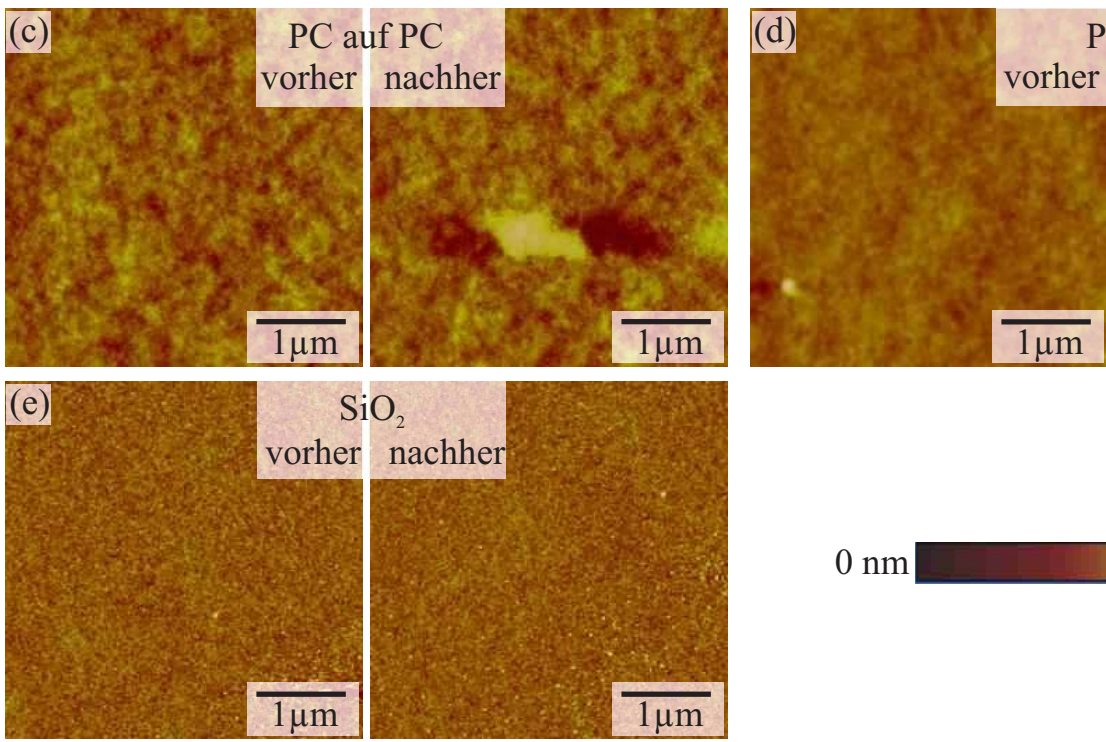

$\mathrm{PC}$

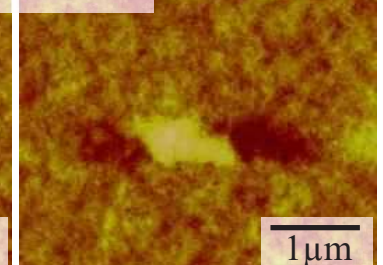

nachher

$0 \mathrm{~nm}$ $5 \mathrm{~nm}$
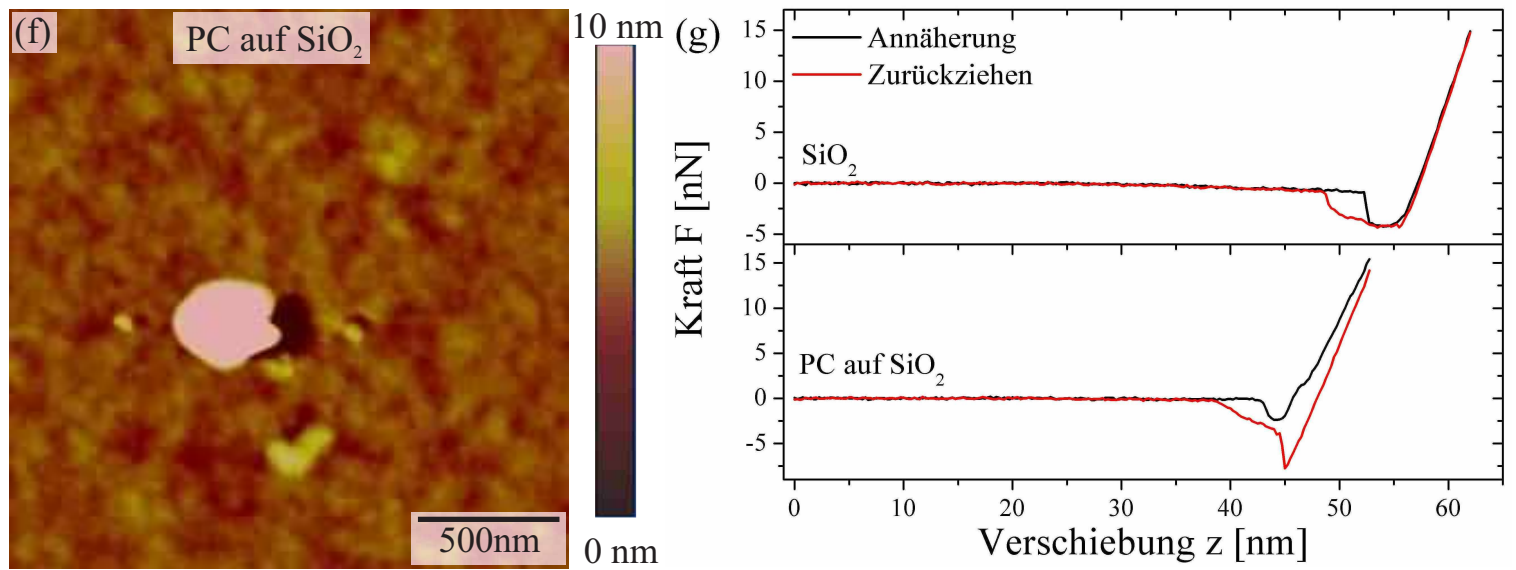

Abbildung 6.21: Vergleicht man die dargestellten Topographien vor und nach der Erstellung einer Karte der lokalen elastischen Eigenschaften, erkennt man auf $\mathrm{SiO}_{2}$ keine Unterschiede (e), während bei allen PC Schichten (aufgedampft und Substrat) Veränderungen sichtbar sind (Höhenunterschiede $\leq 3 \mathrm{~nm}$ (a-d). Führt man Indentationsexperimente mit zu großer Kraft, bzw. maximaler Auslenkung, durch, erhält man viel stärkere Verformungen (f). Der Bereich, in dem die Messung durchgeführt wurde, ist deutlich zu erkennen. In (g) sind zwei typische Kraft-Verschiebungskurven auf $\mathrm{SiO}_{2}$ (oben) und auf PC (unten) dargestellt. Die erkennbare Hysterese zwischen Hin- und Rückweg auf PC ist ein Hinweis auf plastische Verformung. 

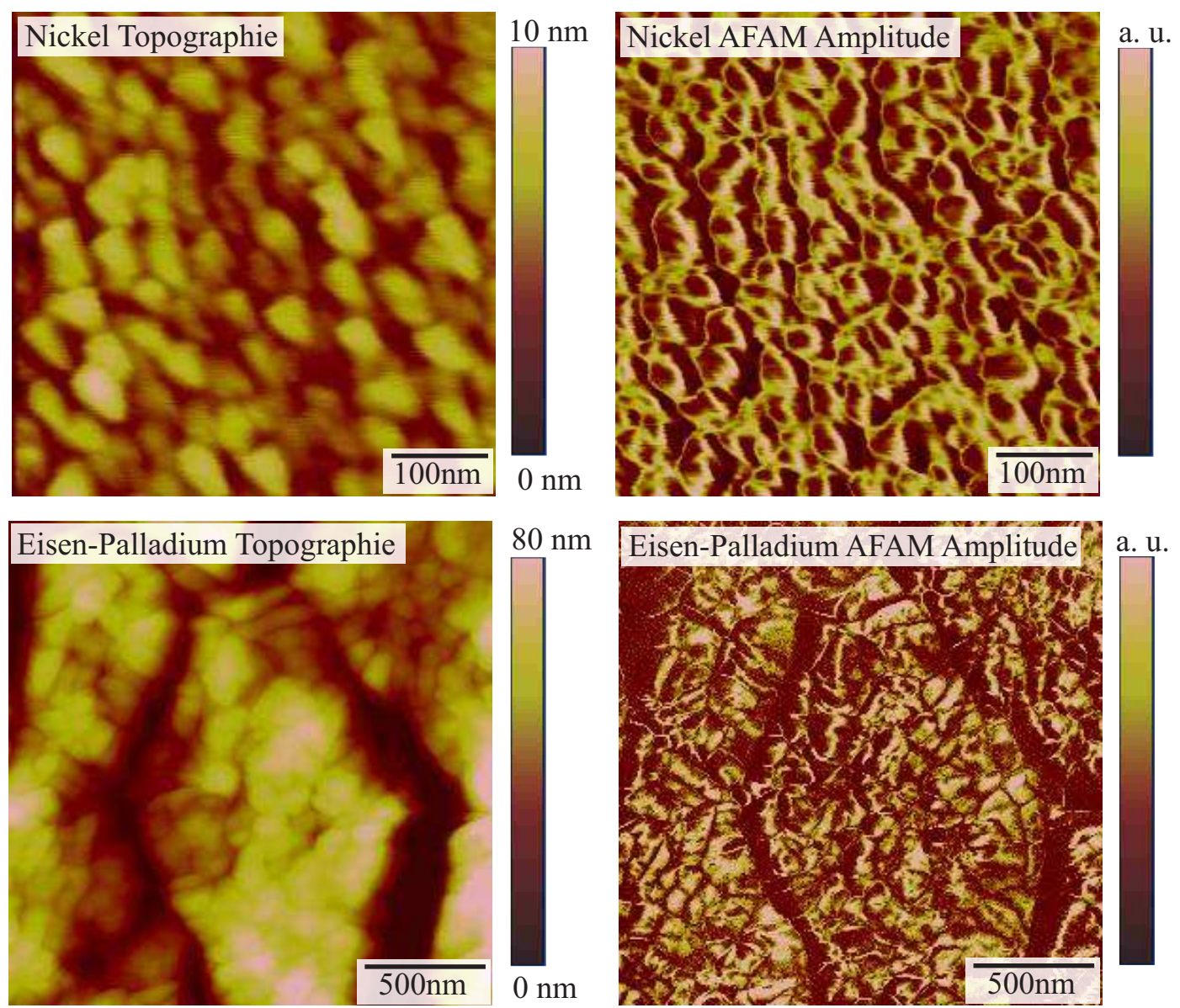

Abbildung 6.22: Beispiele für AFAM Messungen im Bild-Modus an einer Nickel Schicht (oben, vgl. Abb. 3.6) mit $2550 \mathrm{kHz}$ Anregungsfrequenz und an einer Eisen-Palladium Schicht (unten) mit 1350 kHz Anregungsfrequenz. Ein Vergleich der Topographie (jeweils linkes Bild) und der Amplitude (jeweils rechts) zeigt, dass der AFAM Bild-Modus die Kornstruktur deutlicher sichtbar macht.

zen bestimmt worden (vgl. Abs. 3.2.5). Exemplarisch sind je drei Kurven aus diesen Messungen in Abb. 6.23(a) aufgetragen. Die Resonanzfrequenzen liegen für $\mathrm{SiO}_{2}$, welches das Material mit dem niedrigsten Indentationsmodul ist $\left(M_{i s o} \approx 76 \mathrm{GPa}\right)$, am niedrigsten. Es folgen die Kurven für Strontiumtitanat(100) $\left(M_{(100)} \approx 299 \mathrm{GPa}\right)$ und Saphir(101) $\left(M_{(101)} \approx 423 \mathrm{GPa}\right)$. Die Resonanzfrequenzen sind also für härtere Materialien höher. In den Abb. 6.23(b)-(d) sind die Resonanzfrequenzen der aufgenommenen Raster farbcodiert für die einzelnen Materialien aufgetragen. Auf allen Proben variieren die Werte innerhalb eines $20 \mathrm{kHz}$ Bereichs (also unter $2 \%$ ), wobei die Variationen auf $\mathrm{SiO}_{2}$ am größten sind. Kalibriert man einen Cantilever an zwei Referenzproben, kann man aus den Resonanzfrequenzen den Indentationsmodul berechnen. Ein Beispiel dafür ist in Abb. 6.23(e) für Strontiumtitanat(100) (gemessen mit anderem Cantilever, an anderer Stelle und anderer Referenz als in (c)) gezeigt. Mit Werten im Bereich $294 \mathrm{GPa} \leq M \leq 298 \mathrm{GPa}$ sind die Ergebnisse sehr nah am Literaturwert von $M_{S T O\langle 100\rangle} \approx 299 \mathrm{GPa}$. 

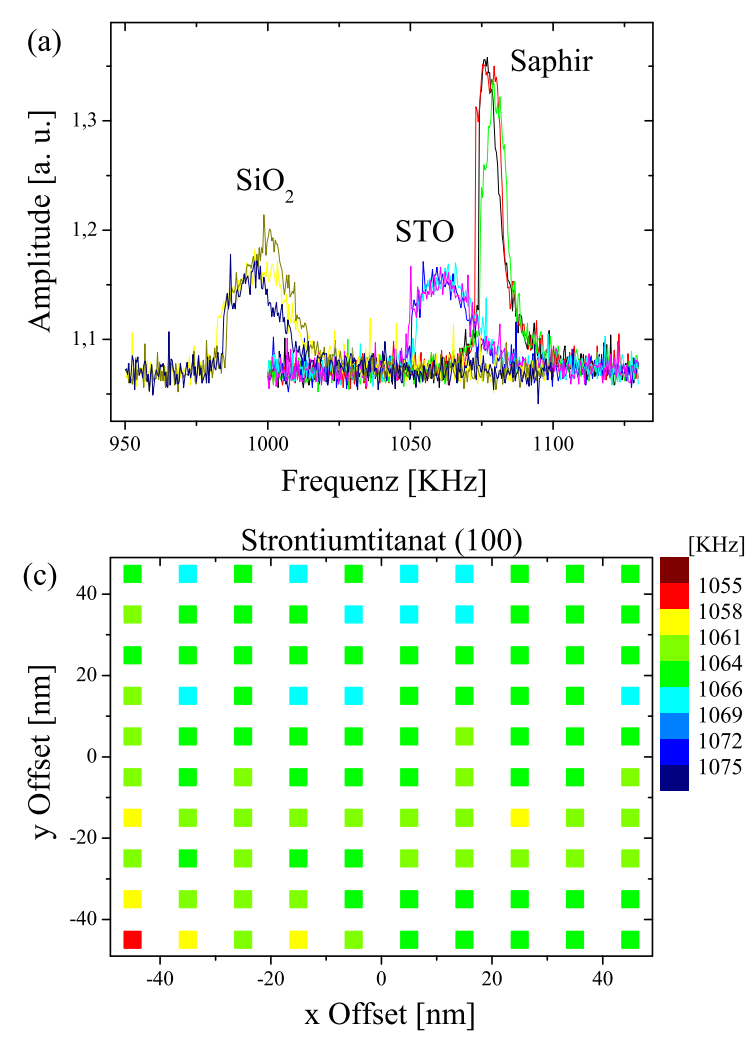
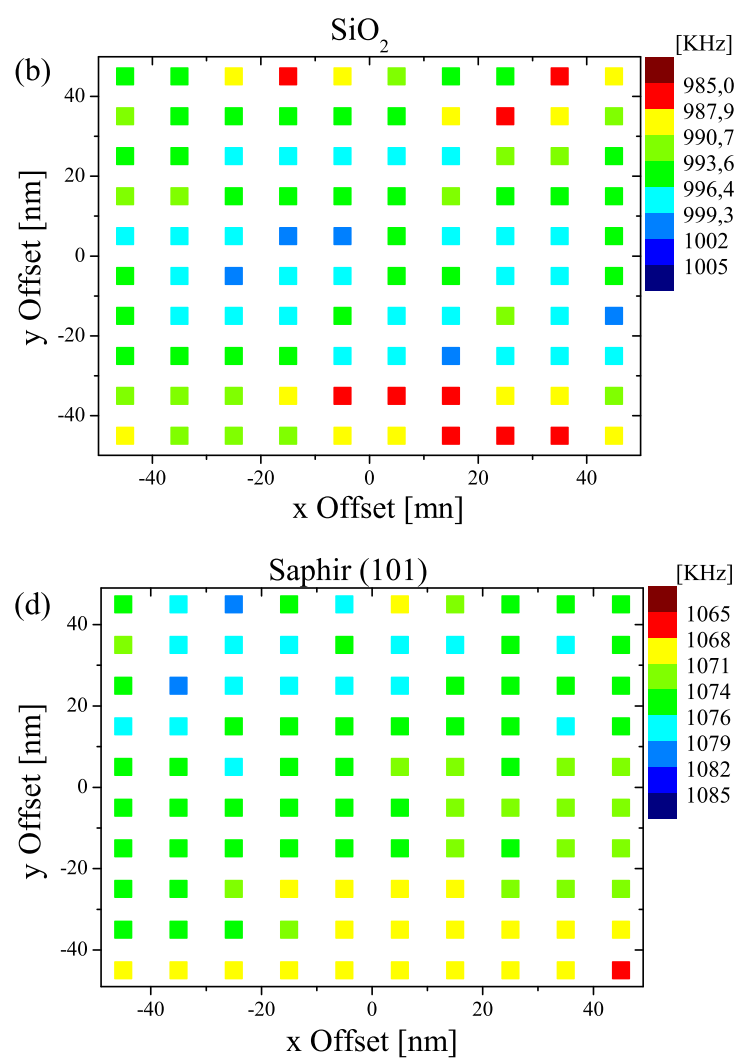

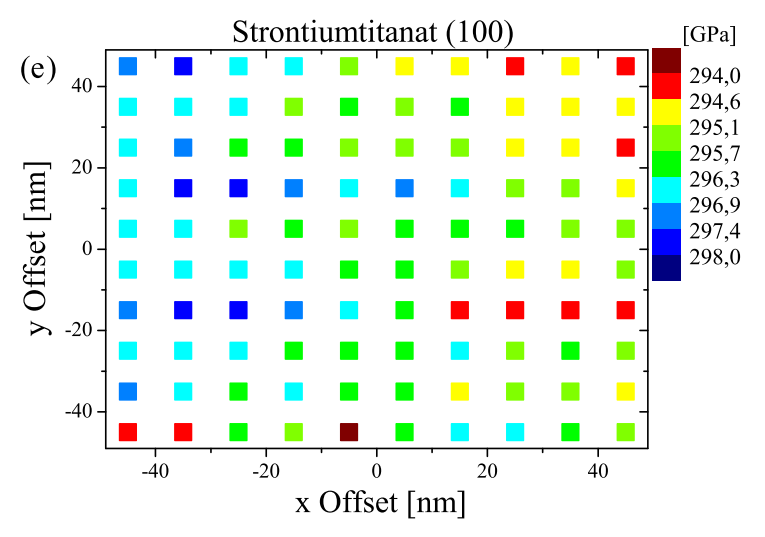

Abbildung 6.23: Beispiele für AFAM Messungen an verschiedenen Kalibrierungsproben. (a) Übersicht über die Lage der Resonanzkurven auf $\mathrm{SiO}_{2}$, Strontiumtitanat(100) (STO) und Saphir(101) anhand von jeweils drei exemplarischen Kurven aus den daneben dargestellten Messungen. Je härter das Material ist, desto höher ist auch die Kontaktsteifigkeit und damit die Resonanzfrequenz. (b)-(d) zeigen Verteilungen der Resonanzfrequenzen anhand eines $10 \times 10$ Punkte Rasters auf den verschiedenen Proben. Auf den kristallinen Proben sind die Variationen etwas geringer als auf dem amorphen $\mathrm{SiO}_{2}$. (e) Verteilung des Indentationsmoduls auf Strontiumtitanat(100), der nach Kalibrierung eines Cantilevers (auf $\mathrm{SiO}_{2}$ und Saphir) aus der Resonanzfrequenz berechnet werden kann (anderer Cantilever und andere Stelle als in (c)). Die Werte liegen nahe am Literaturwert für Massivproben von $M_{S T O(100)} \approx 299 \mathrm{GPa}$. 


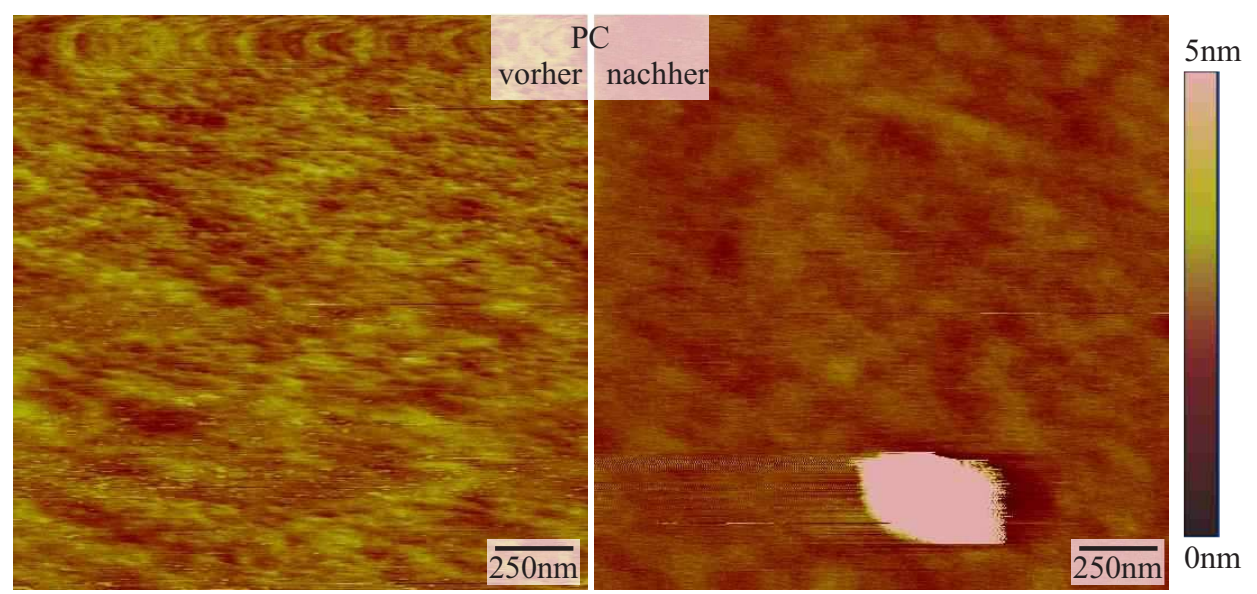

Abbildung 6.24: Topographie eines PC Substrats vor und nach der AFAM Messung. Der Bereich, in dem die Messung durchgeführt worden ist, ist durch die Verformung deutlich zu erkennen.

Führt man solche Messungen an PC Schichten (thermisch verdampft oder Substrat) durch, ergibt sich ein ähnliches Problem wie bei den Messungen mit den KraftAbstandskurven. Um signifikante Resonanzkurven zu erhalten, darf man die Anregungsstärke und die Auflagekräfte nicht beliebig klein wählen. Wie man in Abb. 6.24 an der Topographie eines PC Substrats nach der AFAM Messung sieht, reichen bei den verwendeten Cantilevern die kleinsten möglichen Auflagekräfte, mit denen man noch ein verwertbares Signal erhält, nicht aus, um plastische Verformung durch die Messung zu verhindern. Testweise sind auch Cantilever mit einer Federkonstante von $0,3 \mathrm{~N} / \mathrm{m}$ verwendet worden. Die Verformung der Oberfläche konnte so zwar verringert, aber nicht verhindert werden. 



\section{Ergebnisse der Kontinuumsmodellierung}

In diesem Kapitel sollen Lösungen der in Abs. 4 eingeführten Wachstumsgleichung (4.8) zur Simulation des Wachstums dünner aufgedampfter PC Schichten präsentiert werden. Diese Lösungen liefern ein Höhenprofil und dessen zeitliche Änderung auf dem zugrunde liegenden Gitter $(400 \times 400$ Gitterplätze). Wie in Abs. 4.2.3 beschrieben, kann man das Gitter und das Höhenprofil gemäß $\vec{x} \rightarrow b \vec{x}$ und $h \rightarrow b^{\alpha} h$ reskalieren, um Bildgrößen, die mit experimentellen Ergebnissen vergleichbar sind, zu erhalten. Für den Parameter $b$ wird im Folgenden $b=15$ gewählt, um die Gittergröße auf $6 \times 6 \mu \mathrm{m}^{2}$ zu setzen. Der Rauigkeitsexponent $\alpha$ wird wiederum aus der Höhendifferenzkorrelationsfunktion bestimmt. Um verschiedene Schichten zu vergleichen, wird außerdem wie im Experiment die Schichtdicke und nicht die Zeit als Parameter verwendet.

Da die effektive Hamaker-Konstante der wesentliche Parameter ist, werden die Lösungen für positives und negatives $A$ getrennt voneinander vorgestellt.

\subsection{Modellierung eines instabilen Systems}

Wie in Abs. 4.2.2 diskutiert, führt eine positive Hamaker-Konstante $A>0$ in Gl. (4.8) dazu, dass Lösungen dieser Gleichung Strukturbildung beinhalten. Abb. 7.1 zeigt Topographien dieser Lösungen unter Verwendung der in Tab. 4.1 aufgeführten Parameter für verschiedene Schichtdicken. Auf dem zu Beginn leeren Substrat bilden sich zunächst getrennte Inseln, die mit zunehmender Schichtdicke zusammenwachsen und eine längliche Form annehmen. Zwischen den Inseln verbleiben anfangs grabenartige Vertiefungen, deren Form mit wachsender Dicke immer kreisförmiger wird. Auch bei Schichtdicken $d \geq 200 \mathrm{~nm}$ bleiben diese Formen erkennbar, auch wenn ihre Tiefe immer weiter abnimmt. Damit zusammenhängend ergibt sich ein Rauigkeitsverlauf mit der Schichtdicke wie er in Abb. 7.2 als durchgezogene Linie dargestellt ist. Da der Startpunkt ein glattes, leeres Substrat ist, steigt die Rauigkeit zunächst bis zu einer Schichtdicke $d \approx 21 \mathrm{~nm}$ stark an. Anschließend fällt die Rauigkeit fast ebenso stark wieder ab. Zum Vergleich sind in dieser Abbildung außerdem die experimentellen Daten für PC auf GaAs (Bildgröße $6 \times 6 \mu \mathrm{m}^{2}$ ) aufgetragen. Man erkennt einen sehr ähnlichen Verlauf.

In den Korrelationsfunktionen in Abb. 7.3 findet man für alle Schichtdicken deutliche Maxima. Die Korrelationslänge $R_{c}$ steigt mit zunehmender Schichtdicke von etwa $160 \mathrm{~nm}$ bei $d=20 \mathrm{~nm}$ auf ca. $300 \mathrm{~nm}$ bei $d=100 \mathrm{~nm}$, der Rauigkeitsexponent ist $\alpha \approx 0,8$ für dünne und $\alpha \approx 0,95$ für dickere Schichten. Der Abfall der Fourierspektren für dickere Schichten folgt bei großen $q$ etwa einem $q^{-3}-q^{-4}$ Verlauf, während er für dünne Schichten $d \leq 45 \mathrm{~nm}$ steiler ist. Bei kleineren $q$ wird das Spektrum von dem ausgeprägten zur Korrelationslänge gehörenden Maximum bestimmt. 


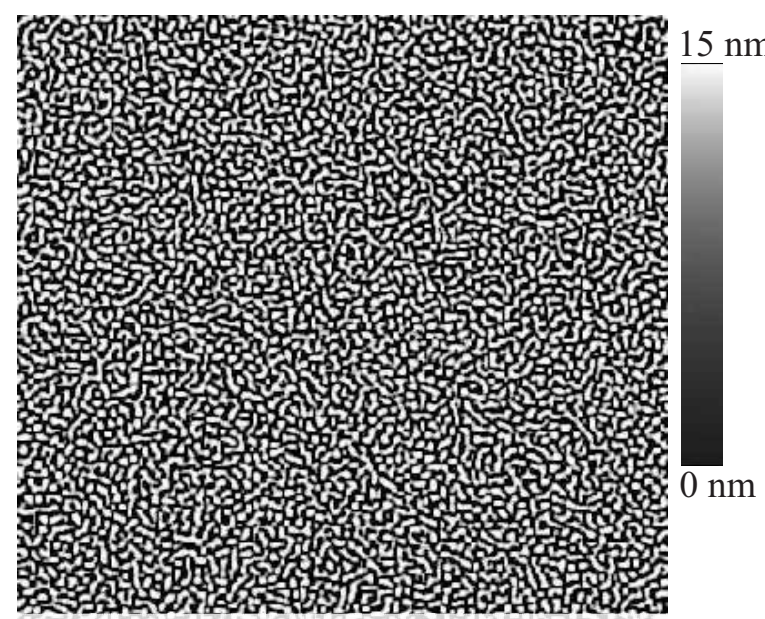

(a) $d=7,5 \mathrm{~nm}, \sigma=6,3 \mathrm{~nm} \quad \overline{1 \mu \mathrm{m}}$
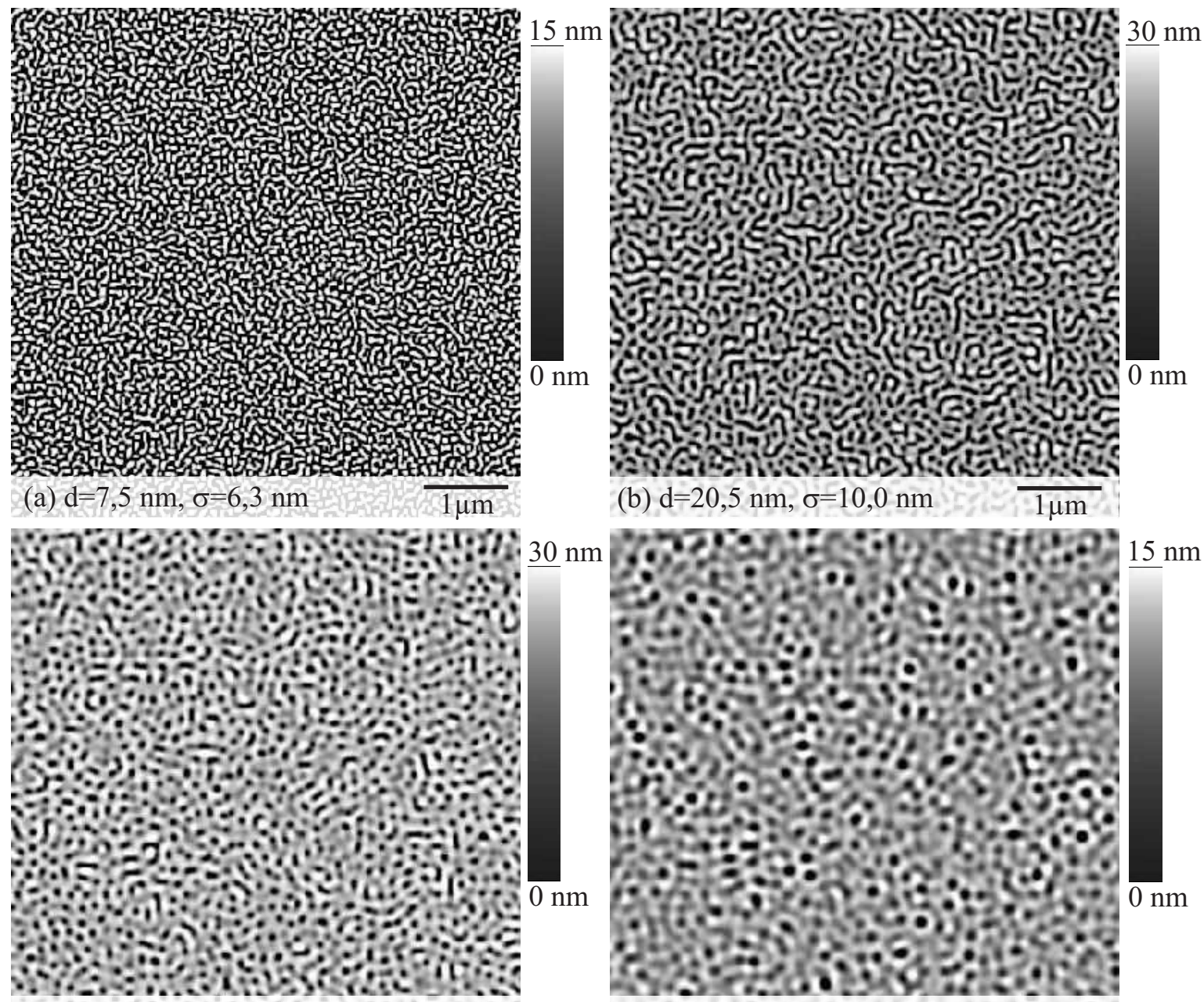

(c) $\mathrm{d}=30,5 \mathrm{~nm}, \sigma=8,4 \mathrm{~nm} \quad 1 \mu \mathrm{m}$

(b) $\mathrm{d}=20,5 \mathrm{~nm}, \sigma=10,0 \mathrm{~nm} \quad \overline{1 \mu \mathrm{m}}$
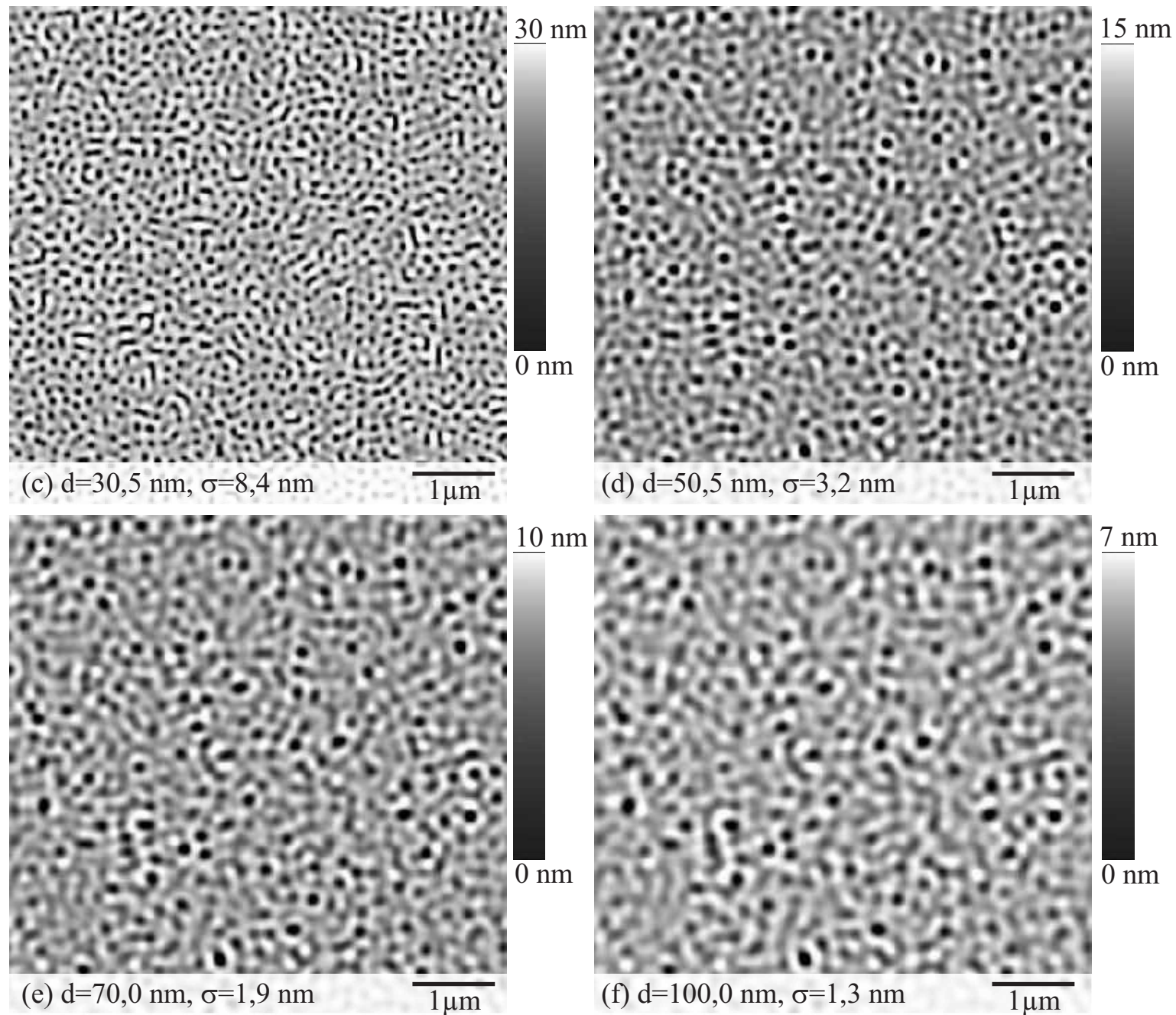

Abbildung 7.1: Topographien für verschiedene Schichtdicken für Lösungen des Kontinuumsmodells Gl. (4.8) mit $A>0(\mathrm{~A}=1,6 \mathrm{nNnm})$. Die Größe der Bildausschnitte beträgt $6 \times 6 \mu \mathrm{m}^{2}$. Zunächst bilden sich längliche getrennte Inseln, die mit zunehmender Schichtdicke zusammenwachsen und immer rundlichere Vertiefungen entstehen lassen. 


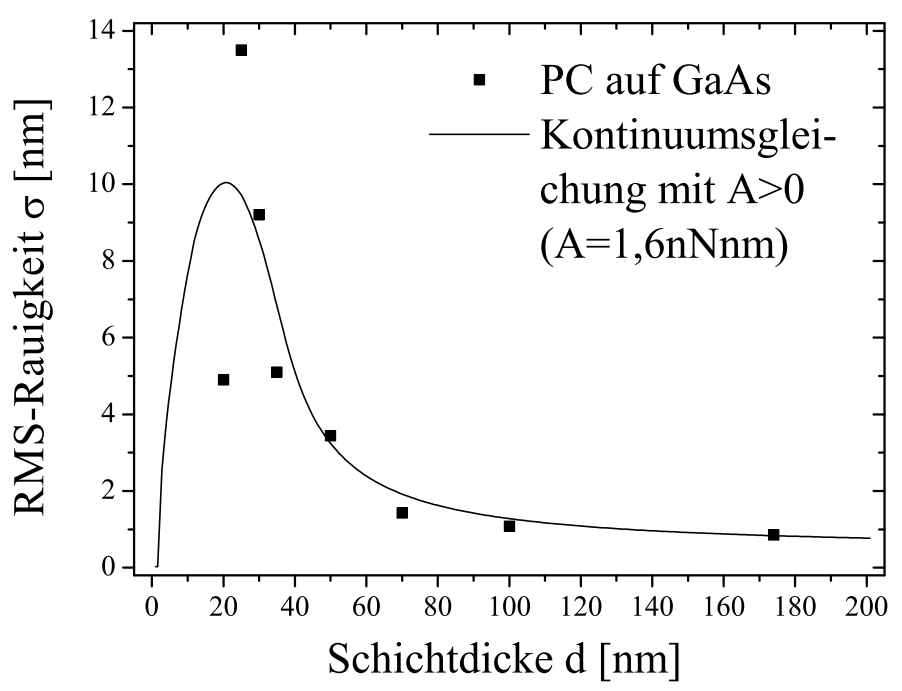

Abbildung 7.2: Rauigkeit in Abhängigkeit von der Schichtdicke für Lösungen des Kontinuumsmodells Gl. (4.8) mit $A>0$ ( $\mathrm{A}=1,6 \mathrm{nNnm}$, durchgezogene Linie). Die Rauigkeit steigt zunächst stark an und nimmt dann mit zunehmender Schichtdicke ab, wodurch sich ein Maximum bei $d \approx 21 \mathrm{~nm}$ bildet. Außerdem sind zum Vergleich noch experimentelle Daten für PC auf GaAs (vgl. Abb. 6.13) gezeigt (Quadrate).
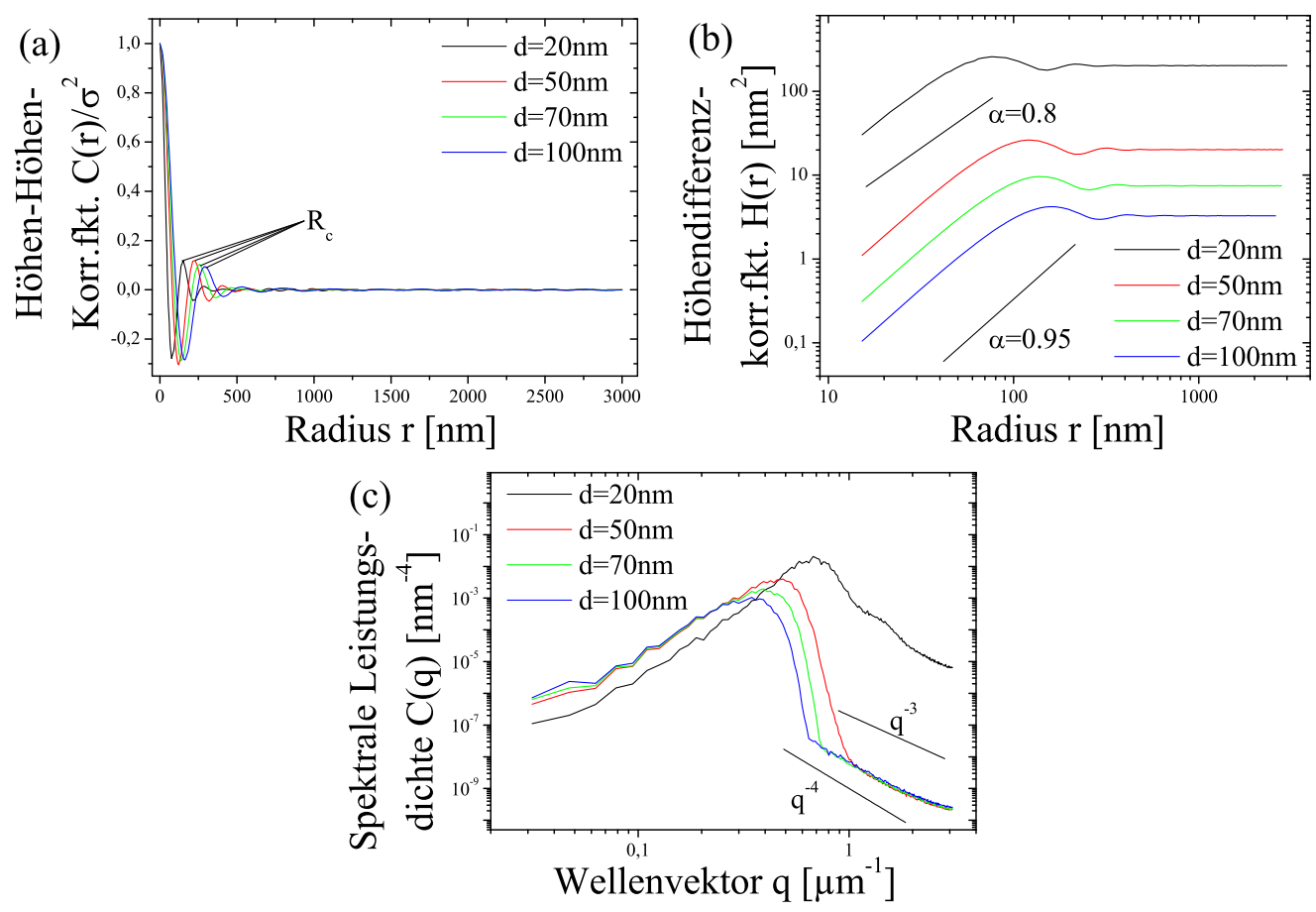

Abbildung 7.3: Korrelationsfunktionen für Lösungen des Kontinuumsmodells Gl. (4.8) mit $A>0(\mathrm{~A}=1,6 \mathrm{nNnm})$ für verschiedene Schichtdicken: (a) Höhen-Höhen-Korrelationsfunktion, (b) Höhendifferenzkorrelationsfunktion und (c) Fourierspektren. Mit zunehmender Schichtdicke wächst die Korrelationslänge $R_{c}$. 


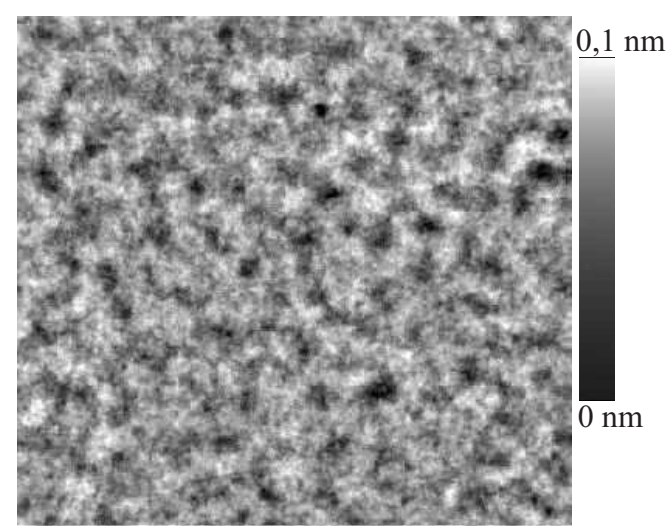

(a) $\mathrm{d}=10 \mathrm{~nm}, \sigma=0,015 \mathrm{~nm}$

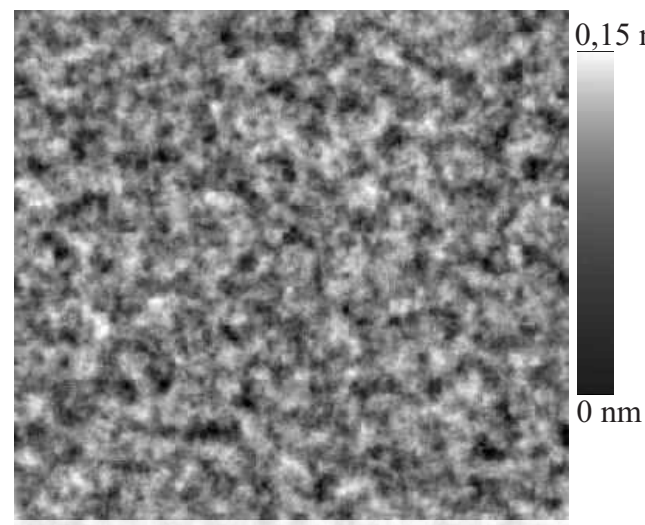

(c) $\mathrm{d}=50 \mathrm{~nm}, \sigma=0,022 \mathrm{~nm}$

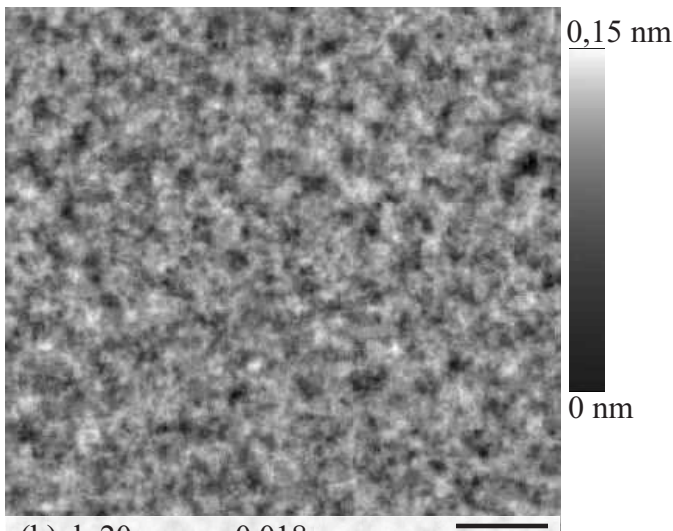

(b) $\mathrm{d}=20 \mathrm{~nm}, \sigma=0,018 \mathrm{~nm}$

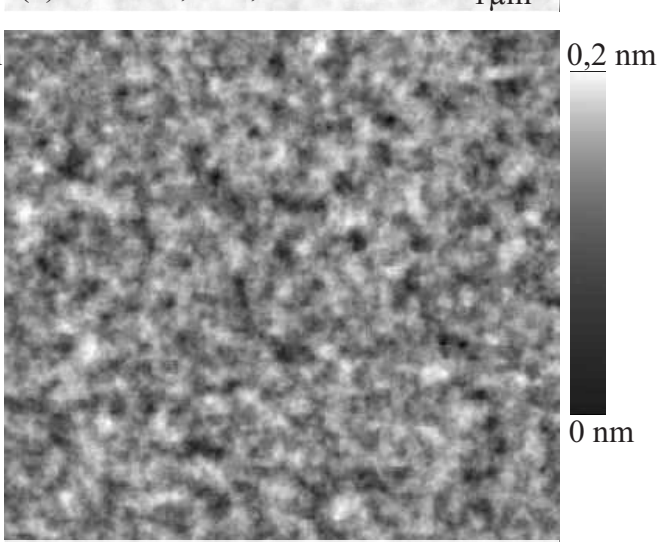

(d) $\mathrm{d}=100 \mathrm{~nm}, \sigma=0,026 \mathrm{~nm}$

$\overline{1 \mu \mathrm{m}}$

Abbildung 7.4: Topographien für verschiedene Schichtdicken für Lösungen des Kontinuumsmodells Gl. (4.8) mit $A<0(\mathrm{~A}=-1,6 \mathrm{nNnm})$. Die Größe der Bildausschnitte beträgt $6 \times 6 \mu \mathrm{m}^{2}$. Alle Schichten sind mit Höhenunterschieden im atomaren Bereich sehr glatt.

\subsection{Modellierung eines stabilen Systems}

Wird Gl. (4.8) mit negativer effektiver Hamaker-Konstante $A<0$ (A=-1,6 nNnm) gelöst, ergeben sich Topographien wie sie für Bildausschnitte der Größe $6 \times 6 \mu \mathrm{m}^{2}$ in Abb. 7.4 für verschiedene Schichtdicken gezeigt sind. Alle Oberflächen sind mit Höhenunterschieden von max. 0,2 nm und Rauigkeiten $\sigma<0,04 \mathrm{~nm}$ atomar glatt. Die Rauigkeit steigt mit zunehmender Schichtdicke von $\sigma=0,015 \mathrm{~nm}$ bei $d=10 \mathrm{~nm}$ leicht auf $\sigma=0,033 \mathrm{~nm}$ bei $d=200 \mathrm{~nm}$ an, wie man an der Rauigkeitsentwicklung in Abb. 7.5 sieht. Die doppelt-logarithmische Auftragung dieser Entwicklung, die im Einsatz in Abb. 7.5 zu sehen ist, zeigt, dass dieser Anstieg einem Potenzgesetz $\sigma \propto d^{0,25}$ folgt. Die Höhen-Höhen-Korrelationsfunktion in Abb. 7.6(a) verläuft für alle Schichtdicken sehr ähnlich, nur dass das erste Minimum mit zunehmender Dicke weniger tief wird. Für $R_{c}$ findet man $R_{c} \approx 500 \mathrm{~nm}$. Abb. 7.6(b) zeigt die Höhendifferenzkorrelationsfunktion. Es ist zu erkennen, dass die Steigung bei kleinen Abständen und damit der Rauigkeitsexponent leicht mit der Schichtdicke ansteigt, dabei gilt $0,6 \leq \alpha \leq 0,7$. Der Abfall der Fourierspektren (Abb. 7.6(c)) folgt für große Wellenzahlen etwa einem $q^{-4}$ Verlauf. 


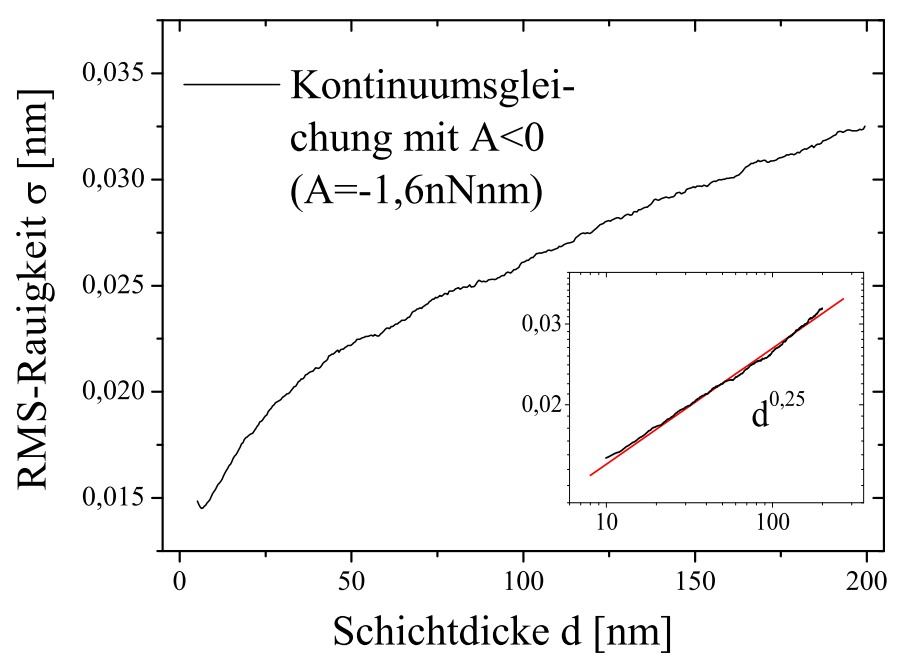

Abbildung 7.5: Rauigkeit in Abhängigkeit von der Schichtdicke für Lösungen des Kontinuumsmodells Gl. (4.8) mit $A<0(\mathrm{~A}=-1,6 \mathrm{nNnm})$. Der Einsatz zeigt dieselben Daten in doppelt-logarithmischer Auftragung. Die Rauigkeit steigt über den gesamten Dickenbereich schwach an und folgt einem Potenzgesetz $\sigma \propto d^{0,25}$.
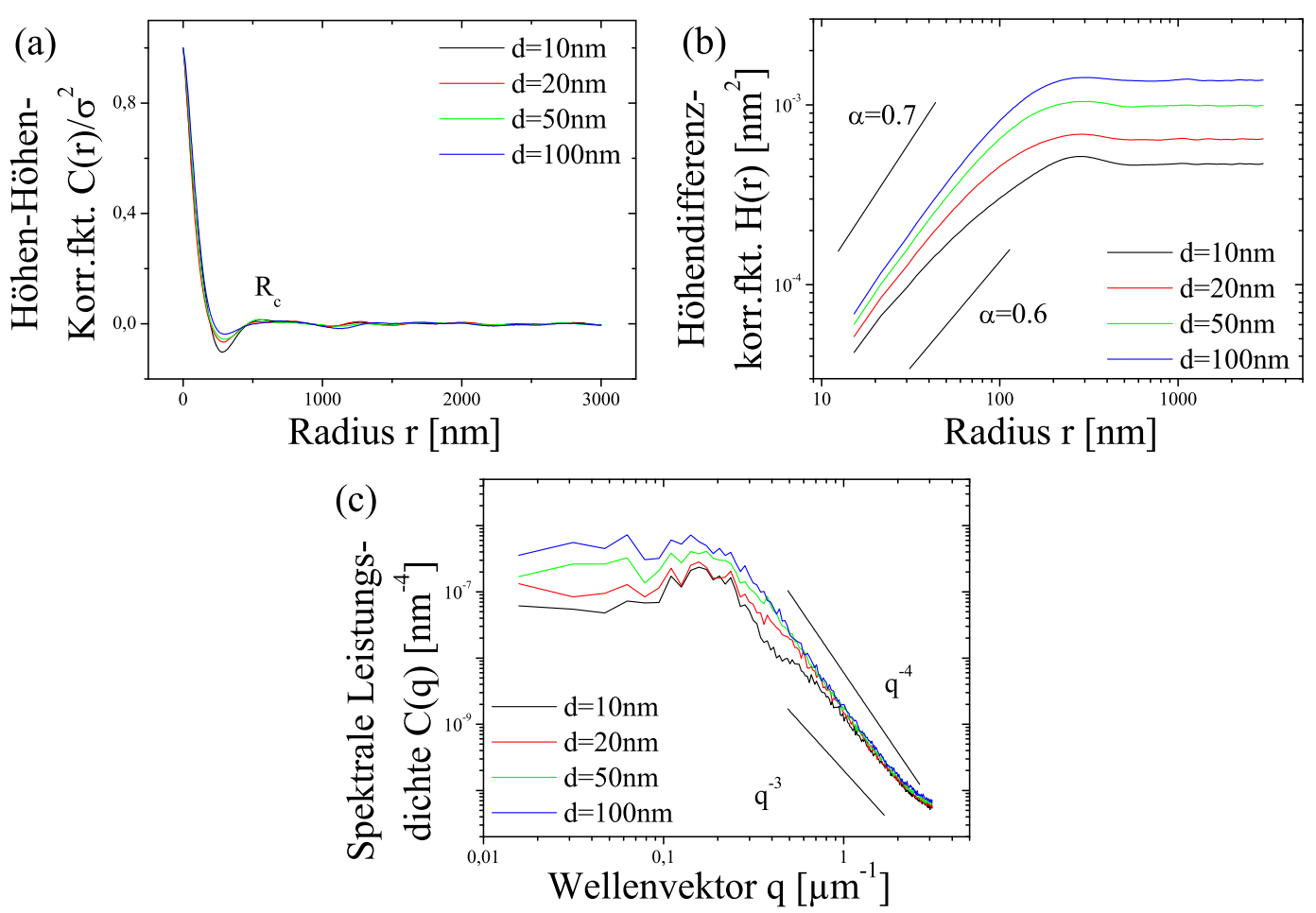

Abbildung 7.6: Korrelationsfunktionen für Lösungen des Kontinuumsmodells Gl. (4.8) mit $A<0(\mathrm{~A}=-1,6 \mathrm{nNnm})$ für verschiedene Schichtdicken: (a) Höhen-Höhen-Korrelationsfunktion, (b) Höhendifferenzkorrelationsfunktion und (c) Fourierspektren. Die Korrelationslänge ändert sich kaum mit der Schichtdicke. 



\section{Ergebnisse der MD Simulationen}

In diesem Kapitel sollen die Ergebnisse der MD Simulationen vorgestellt werden. Aus den zugänglichen Positionen und Geschwindigkeiten zu jeder Zeit $t$ können viele Eigenschaften berechnet werden. Man erhält eine große Ergebnisvielfalt, die an dieser Stelle nicht vollständig vorgestellt werden kann. Daher werden nur die wichtigsten Ergebnisse präsentiert. Zunächst soll verifiziert werden, dass die präparierten Zellen amorph und ausreichend relaxiert sind, bevor die Dynamik einer einzelnen Kette auf der Oberfläche einer Simulationszelle analysiert wird. Anschließend wird der Einfluss der Oberflächen auf die Dynamik eingehend betrachtet.

Alle Ergebnisse werden, wie in Abs. 5.2.1 beschrieben, in Lennard-Jones Einheiten dargestellt.

\subsection{Amorphe Struktur und Glasübergang}

Da ein Ziel dieser Arbeit die Untersuchung der Dynamik von Polymeren im Bereich des Glasübergangs ist, muss zunächst untersucht werden, ob die Struktur der hergestellten Zellen amorph ist und ob, bzw. bei welcher Temperatur die Zellen einen Glasübergang aufweisen. Wie in Abs. 2.1.1 erklärt, ist die Paarkorrelationsfunktion ein gutes Maß, um die Abwesenheit von langreichweitiger Ordnung zu verdeutlichen. Eine typische Paarkorrelationsfunktion aus der Simulation ist in Abb. 8.1(a) für eine Probe bei $T=0,4 \epsilon / \mathrm{k}_{\mathrm{B}}$ gezeigt. Bei einem Abstand von $r=1,1 \sigma$ ist ein scharfes Maximum zu erkennen, das den mittleren Nächsten-Nachbar Abstand angibt. Die weiteren Maxima beim Übernächsten-Nachbar Abstand, beim Über-Übernächsten usw. nehmen stark an Intensität ab, so dass der siebte Ausschlag bei $r \approx 6,3 \sigma$ kaum noch zu erkennen ist. Außerdem sind sie verbreitert, wie man es für Flüssigkeiten und amorphe Systeme erwartet (vgl. Abs. 2.1.1). Des Weiteren ist während des Abkühlens (und während des Heizens) die Signatur eines Glasübergangs zu erkennen. Dies äußert sich z. B. in der Volumenänderung mit der Temperatur, die für verschiedene Abkühlraten von $\frac{9 \epsilon / \mathrm{k}_{\mathrm{B}}}{10^{6} \Delta \mathrm{t}}$ bis $\frac{9 \epsilon / \mathrm{k}_{\mathrm{B}}}{10^{8} \Delta \mathrm{t}}$ exemplarisch in Abb. 8.1(b) gezeigt ist. Aus diesen Messungen kann, wie in Abs. 2.1.1 beschrieben, die Glasübergangstemperatur $T_{g}$ bestimmt werden. Je nach Abkühlrate findet man Werte zwischen $T_{g} \approx 0,42 \epsilon / \mathrm{k}_{\mathrm{B}}$ bei der langsamsten von den getesteten Abkühlraten und $T_{g} \approx 0,46 \epsilon / \mathrm{k}_{\mathrm{B}}$ bei der schnellsten. $T_{g}$ steigt dabei wie erwartet kontinuierlich mit zunehmender Abkühlrate.

Neben der Verifizierung der amorphen Struktur soll getestet werden, ob die Relaxation nach der Herstellung ausreicht, um einen definierten Anfangs- bzw. Referenzzustand zu haben. Ist die Relaxationszeit zu kurz, kann das System seine Gesamtenergie in der Folge weiter verringern. Wie man im Verlauf der Gesamtenergie über die Simulationszeit in Abb. 8.2(a) für eine Simulation bei $T=0,40 \epsilon / \mathrm{k}_{\mathrm{B}}$ sehen kann, bleibt die Gesamtenergie innerhalb statistischer Fluktuationen jedoch konstant. Um die Relaxa- 
(a)

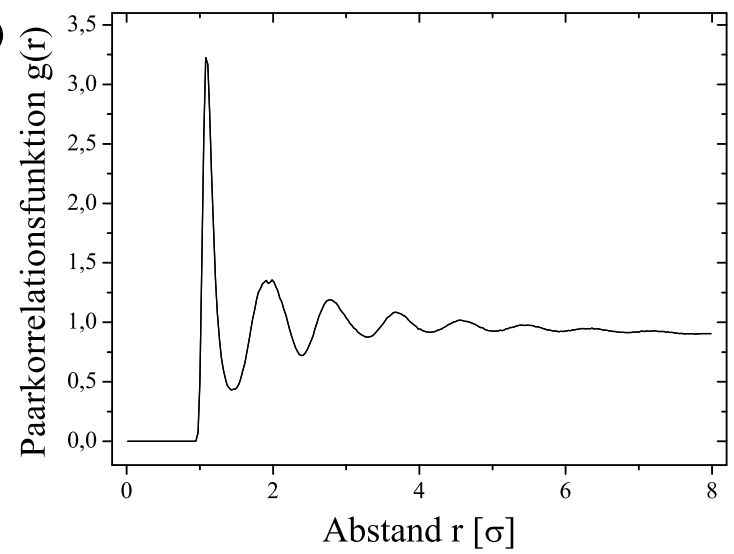

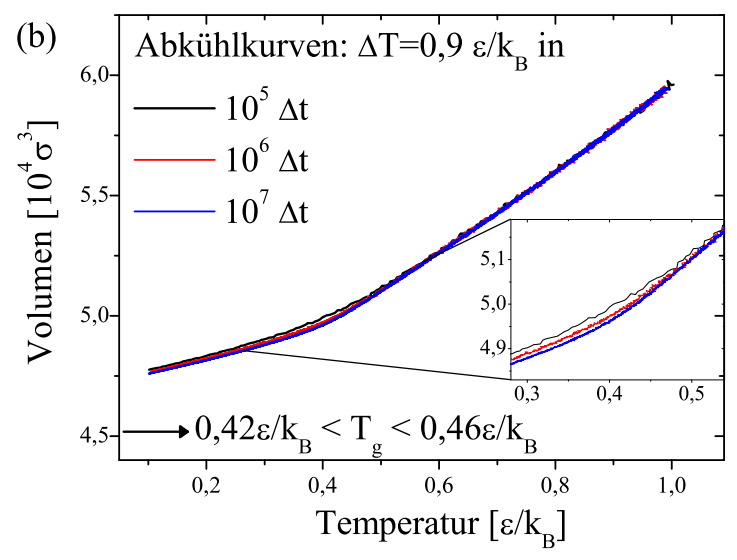

Abbildung 8.1: (a) Die Paarkorrelationsfunktion $g(r)$ einer Simulationszelle (hier für $T=0,4 \epsilon / \mathrm{k}_{\mathrm{B}}$ ) zeigt den typischen Verlauf eines Glasbildners (vgl. Abs. 2.1.1). (b) Volumenänderung mit der Temperatur während des Abkühlens mit verschiedenen Raten. Im Bereich der Glasübergangstemperatur ändert sich die Steigung deutlich. Je nach Abkühlrate erhält man Glasübergangstemperaturen von $0,42 \epsilon / \mathrm{k}_{\mathrm{B}} \lesssim T_{g} \lesssim 0,46 \epsilon / \mathrm{k}_{\mathrm{B}}$.

tionszeit weiter zu prüfen, ist in einem Testlauf die Relaxationszeit nach dem Heizen auf die gewünschte Temperatur (vgl. Abs. 5.2.4) verdoppelt worden. Diese verlängerte Relaxationszeit hat innerhalb der Fehler zu gleichen Ergebnissen geführt. Um den Einfluss des Zerschneidens bei der Präparation der Zellen nach Variante $1 \mathrm{zu}$ testen, ist in Abb. 8.2(b) eine typische Kettenlängenverteilung aufgetragen. Es ist zu erkennen, dass ca. $90 \%$ der anfänglich 1730 Ketten unverändert bleiben. Abb. 8.2(c) zeigt den Gyrationsradius und den End-zu-End Abstand für die verschiedenen Kettenlängen. Für alle Längen gilt in guter Näherung $R_{E}^{2}=6 R_{G}^{2}$, was für lineare ungestörte Ketten zu erwarten ist, d. h. die Relaxationszeit ist lang genug.

\subsection{Dynamik einer einzelnen Kette auf der Oberfläche}

Alle in diesem Abschnitt untersuchten Proben sind nach Variante 1 (vgl. Abs. 5.2.2) hergestellt worden. Von den insgesamt zehn verschiedenen Startkonfigurationen, die bei $T=0,40 \epsilon / \mathrm{k}_{\mathrm{B}}$ und $T=0,44 \epsilon / \mathrm{k}_{\mathrm{B}}$ untersucht worden sind, wird im Folgenden eine Simulation bei $T=0,40 \epsilon / \mathrm{k}_{\mathrm{B}}$ ausführlich vorgestellt. Qualitativ weisen alle Simulationen das gleiche Verhalten auf. Anschließend wird der Einfluss der Temperatur anhand eines Beispiels verdeutlicht.

In Abb. 8.3 ist die Bewegung der Kette auf der Oberfläche durch Momentaufnahmen der Simulationszelle zu verfolgen. Die anfangs gerade Kette zieht sich zunächst zusammen, da der gestreckte Zustand entropisch ungünstig ist. Dieser Startpunkt ist als Referenzpunkt für die mittleren Verschiebungsweiten ungeeignet, weshalb für diese ein späterer Zeitpunkt $t_{1}>t_{0}$ als Referenz gewählt wird. Außerdem driftet die gesamte Zelle in einigen Simulationsdurchläufen etwas, daher werden alle Positionen um die 

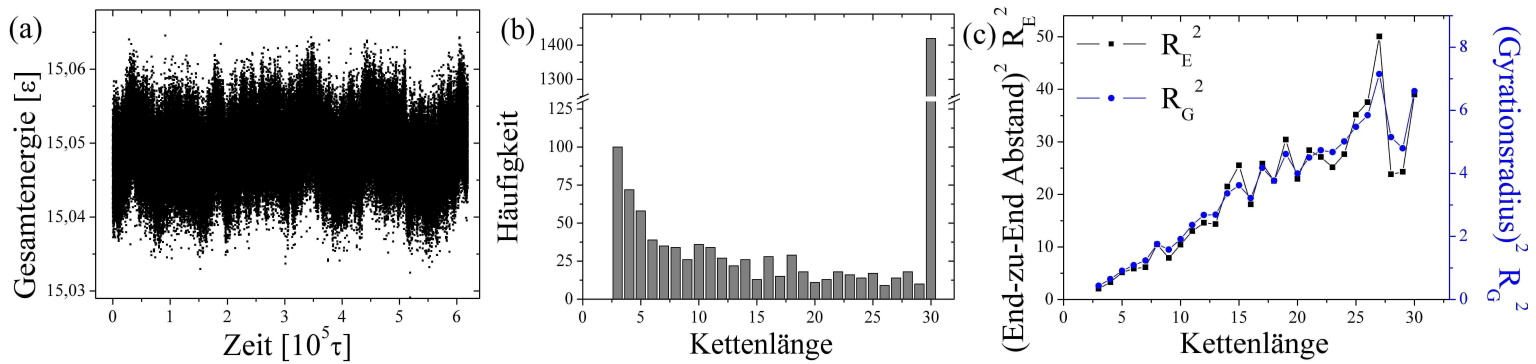

Abbildung 8.2: (a) Zeitlicher Verlauf der Gesamtenergie während einer typischen Simulation bei $T=0,40 \epsilon / \mathrm{k}_{\mathrm{B}}$. Bis auf statistische Fluktuationen bleibt die Gesamtenergie über den gesamten Bereich konstant. (b) Typische Kettenlängenverteilung für eine nach Variante 1 hergestellte Simulationszelle. Die y-Achse ist für eine bessere Lesbarkeit unterbrochen dargestellt, da die meisten Ketten nicht zerschnitten werden. (c) Vergleich von (quadratischem) Gyrationsradius $R_{G}^{2}$ und (quadratischem) End-zu-End Abstand $R_{E}^{2}$ in Abhängigkeit von der Kettenlänge für eine nach Variante 1 hergestellte Simulationszelle, also insbesondere eine Zelle mit zerschnittenen Ketten. Für alle Kettenlängen gilt in guter Näherung $R_{E}^{2}=6 R_{G}^{2}$, was für eine genügend lange Relaxationszeit spricht (vgl. Abs. 2.2).

(sehr kleine) Bewegung des Schwerpunktes der gesamten Zelle korrigiert.

Wie man z. B. zum Zeitpunkt $t_{0}+1 \times 10^{4} \tau$ sehen kann, wird die Kette zwar auf der Oberfläche platziert, aber natürlich ist es ihr auch erlaubt, sich in die Zelle hinein zu bewegen. Da das System jedoch unterhalb der Glasübergangstemperatur gehalten wird, ist die Zelle ein Festkörper und das Eindiffundieren ist entsprechend langsam. Oberhalb von $T_{g}$ ist die Kette schon nach kurzer Zeit in der Zelle „,verschwunden“, also nicht mehr an der Oberfläche zu sehen.

In Abb. 8.4 sind der zeitliche Verlauf von End-zu-End Abstand (a) und Gyrationsradius (b) der einzelnen Kette (schwarz) im Vergleich zum Mittel der gesamten Zelle (rot, vergrößert im Einsatz) bei $T=0,40 \epsilon / \mathrm{k}_{\mathrm{B}}$ dargestellt. Bei der Betrachtung einer einzelnen Kette erkennt man stets große Schwankungen, die auch bei den mittleren Verschiebungsweiten, die in Abb. 8.5(a) für die einzelnen Monomere und (b) für den Kettenschwerpunkt dargestellt sind, deutlich werden. Es gibt Zeiträume, in denen sich die Kette viel bewegt, aber auch welche, in denen sich die MSD kaum ändern. Betrachtet man die Schwerpunktbewegung in Abb. 8.5(b), fällt auf, dass sich die Kette teilweise weit von ihrem Ausgangspunkt entfernt, sich zu anderen Zeiten aber auch wieder diesem Punkt annähert, es gibt also - wie man es bei einer zufälligen Bewegung erwartet keine ausgezeichnete Richtung. Erst durch eine Mittelung über viele Ketten (oder sehr lange Zeiten) kann man quantitative Aussagen über die Diffusionsbewegung machen und daher ist eine Berechnung der effektiven Diffusionskonstante für die einzelne Kette hier nicht möglich. Dies wird bei Betrachtung der mittleren Verschiebungsweiten aller Ketten der Länge 30 in der Zelle sichtbar, die in Abb. 8.5(c) sowohl für die Mittelung über alle Monomere als auch für die Mittelung der Schwerpunktbewegungen gezeigt ist. Hier erkennt man auch deutlich, dass die Schwerpunktbewegung der Ketten den 

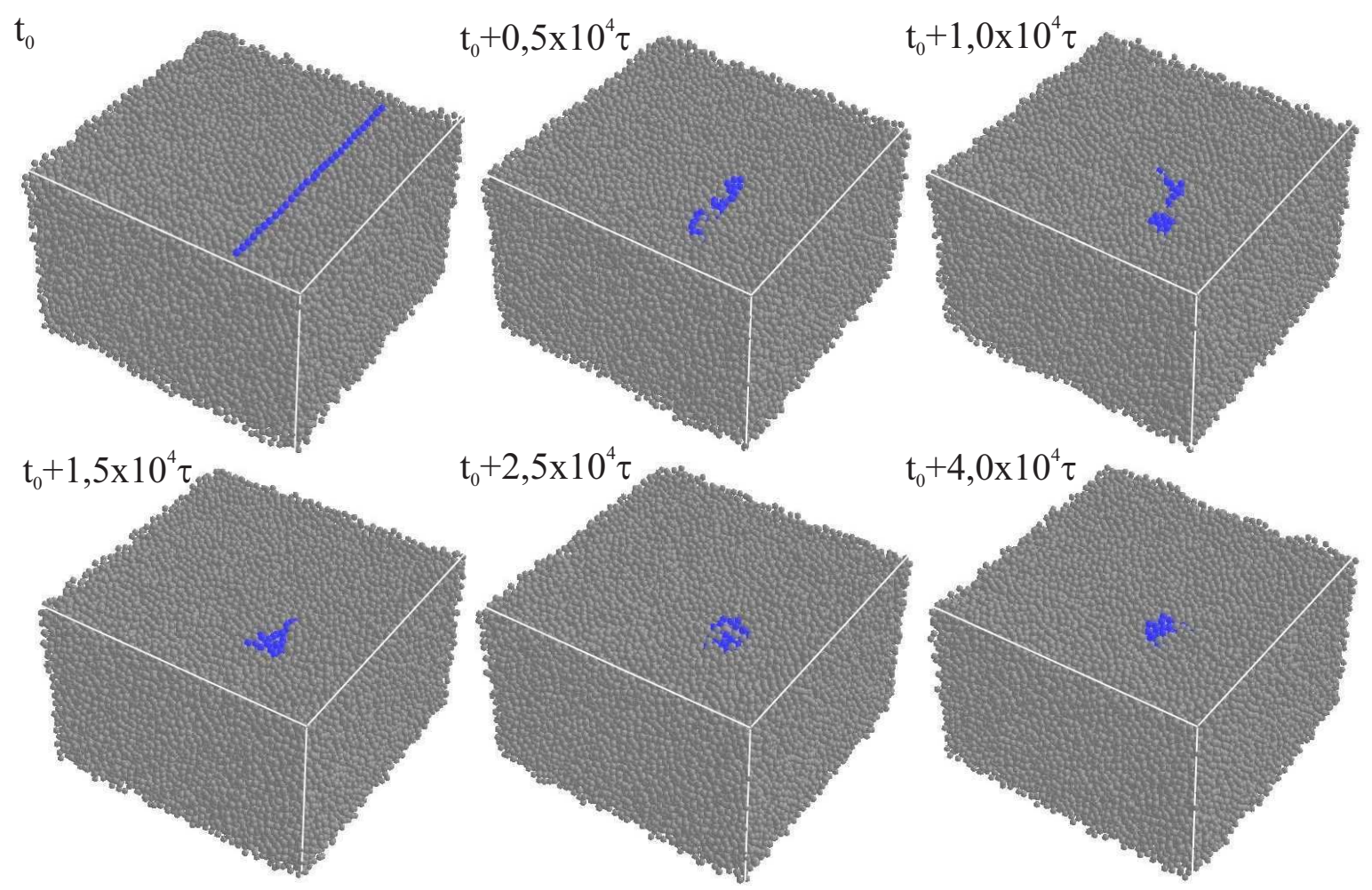

Abbildung 8.3: Momentaufnahmen einer Simulation mit einer Kette auf der Oberfläche. Zum Zeitpunkt $t_{0}$ wird die lineare gerade Kette auf der Oberfläche platziert. Innerhalb der ersten ca. $10^{3} \tau$ zieht sich die Kette zusammen.

diffusiven Bereich deutlich früher als die Monomerbewegung erreicht (vgl. Abs. 2.2).

In Abb. 8.6 sind zum Vergleich die MSDs einer Simulation bei $T=0,44 \epsilon / \mathrm{k}_{\mathrm{B}}$ gezeigt. Für die Volumendiffusion in Abb. 8.6(c) ergibt sich ein zu den Ergebnissen bei $T=$ $0,40 \epsilon / \mathrm{k}_{\mathrm{B}}$ qualitativ gleicher Verlauf, wobei die absoluten Verschiebungsweiten deutlich größer sind. Betrachtet man die Einzelkettenbewegung, unterscheiden sich die Verhalten bei den beiden Temperaturen deutlicher. Es sind zwar auch bei $T=0,44 \epsilon / \mathrm{k}_{\mathrm{B}}$ große Schwankungen zu erkennen, aber darüber hinaus gibt es eine effektive Bewegung weg vom Startpunkt. Allerdings unterscheiden sich diese Bewegungen von Simulation zu Simulation in ihrer Größse deutlich, so dass ohne eine effektive Mittelung wiederum kaum quantitative Aussagen getätigt werden können.

Durch die starken Fluktuationen ist es somit schwierig, quantitative Aussagen über die Einzelkettenbewegung zu machen, da die absoluten Werte der MSD sehr unterschiedlich sein können. Erst durch eine Mittelung über geeignet viele Ketten kann man signifikante quantitative Aussagen tätigen. Daher wird im Folgenden nicht mehr eine einzelne Kette betrachtet, sondern alle Ketten in der Nähe der Oberfläche im Vergleich zu den Ketten im Zentrum der Zelle. 

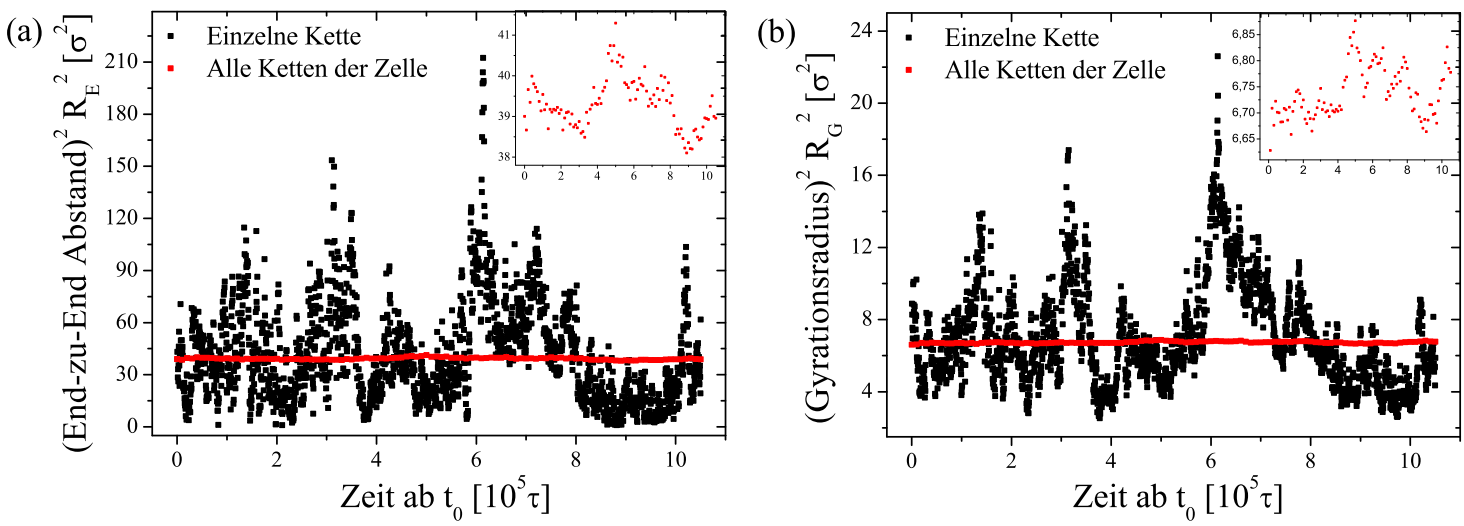

Abbildung 8.4: Zeitlicher Verlauf des End-zu-End Abstands (a) und des Gyrationsradius (b) einer einzelnen auf der Oberfläche platzierten Kette (schwarz) und des Mittels aller Ketten der Länge 30 in der Simulationszelle (rot) bei $T=0,40 \epsilon / \mathrm{k}_{\mathrm{B}}$. Vergrößert dargestellt sind jeweils die Daten aus den Mittelwerten aller Ketten.

(a)

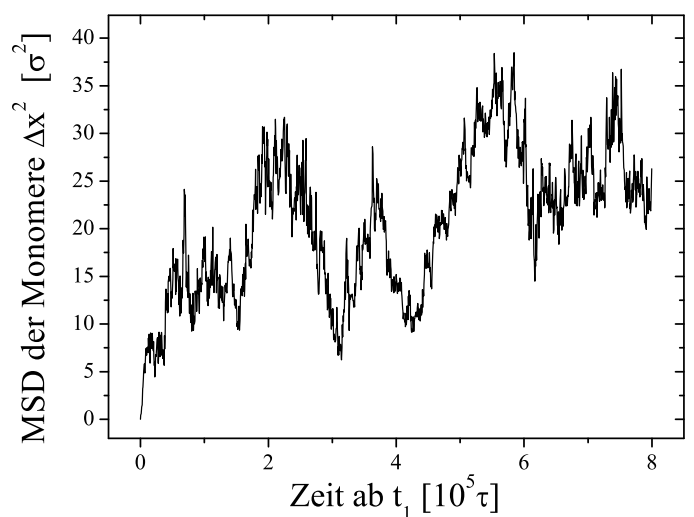

(b)

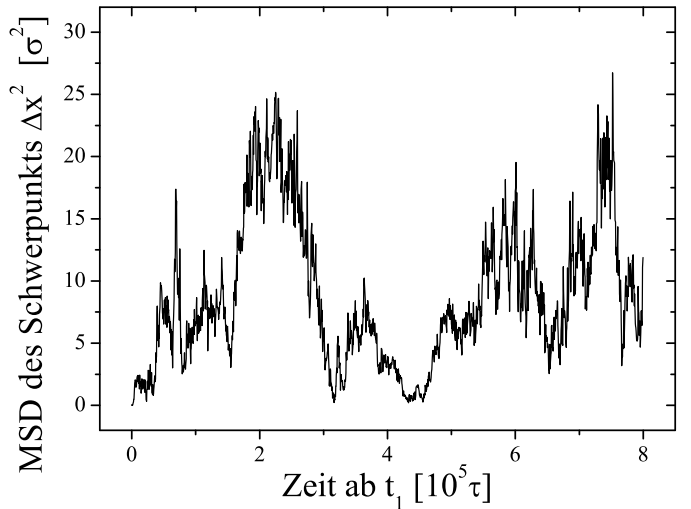

(c)

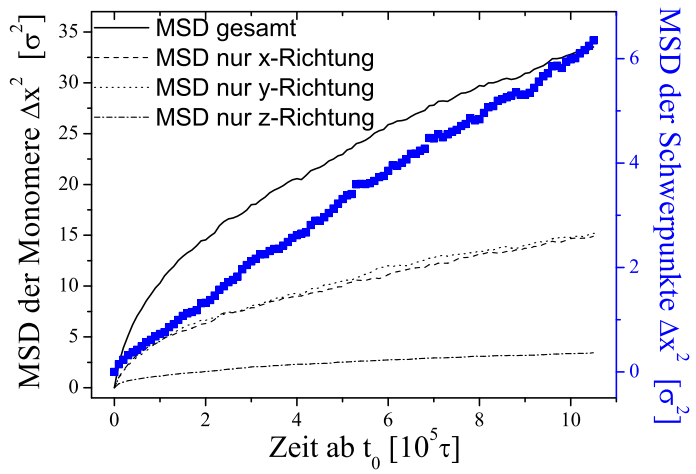

Abbildung 8.5: Zum Vergleich der Bewegung einer einzelnen Kette auf der Oberfläche mit den übrigen Ketten bei $T=0,40 \epsilon / \mathrm{k}_{\mathrm{B}}$ sieht man in (a) die MSD der Monomere der einzelnen Kette, (b) die MSD des Kettenschwerpunkts und (c) die gleichen Werte für das Mittel des gesamten Ensembles. Für die Einzelkettenbewegung ist der Zeitpunkt $t_{1}=t_{0}+0,25 \times 10^{4} \tau$ als Referenzzustand gewählt. Die Bewegung der einzelnen Kette ist durch große Schwankungen dominiert. 
(a)

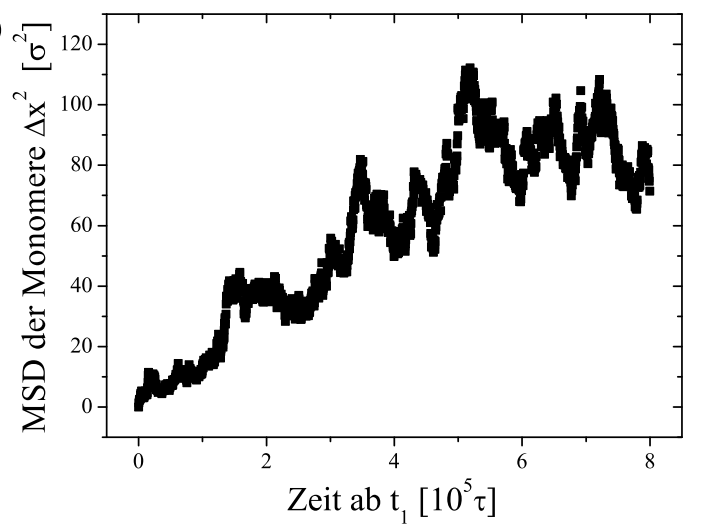

(b)

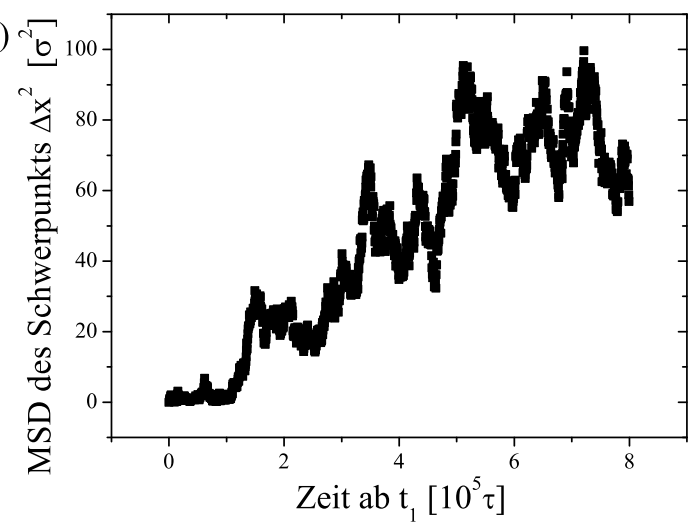

(c)

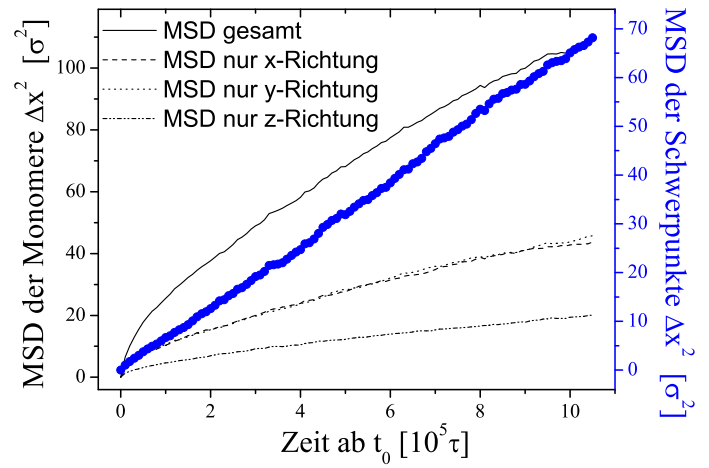

Abbildung 8.6: Zum Vergleich der Bewegung einer einzelnen Kette auf der Oberfläche mit den übrigen Ketten bei $T=0,44 \epsilon / \mathrm{k}_{\mathrm{B}}$ sieht man in (a) die MSD der Monomere der einzelnen Kette, (b) die MSD des Kettenschwerpunkts und (c) die gleichen Werte für das Mittel des gesamten Ensembles. Für die Einzelkettenbewegung ist der Zeitpunkt $t_{1}=t_{0}+0,25 \times 10^{4} \tau$ als Referenzzustand gewählt. Die Bewegung der einzelnen Kette weist bei dieser Temperatur ebenfalls große Schwankungen auf, dennoch ist eine deutliche Steigung erkennbar. 


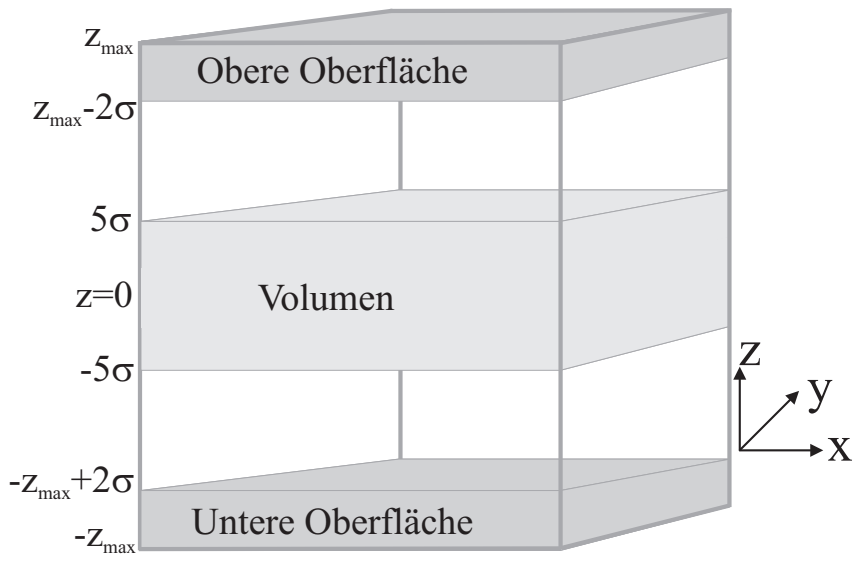

Abbildung 8.7: Zur Veranschaulichung der Definition, welche Bereiche einer Zelle zur Oberfläche und welche zum Volumen gezählt werden, sind diese Bereiche in der schematischen Darstellung markiert. Für das Volumen werden die mittleren $10 \sigma$ betrachtet, während der Oberflächenbereich jeweils die oberen und unteren $2 \sigma$ beinhaltet.

\subsection{Einfluss der Oberflächen auf die Dynamik}

Um den Einfluss von freien Oberflächen auf die Dynamik der Ketten zu untersuchen, werden im Folgenden die z-Koordinaten des Schwerpunktes einer Kette zu Beginn der Simulation verwendet, um die Nähe zur Oberfläche festzulegen. Oberflächeneffekte, also Änderungen nahe der Oberfläche im Vergleich zum Volumen, werden durch Betrachtung der z-Abhängigkeit einiger Größen, z. B. der effektiven Diffusionskonstante (vgl. Gl. (2.4) in Abs. 2.2), oder durch getrennte Mittelung innerhalb der Oberflächenbereiche und des Volumenbereichs sichtbar. Als Oberflächenbereich werden hier die oberen und unteren $2 \sigma$ der Simulationszelle betrachtet, während sich der Volumenbereich über die mittleren $10 \sigma$ erstreckt, wie es in Abb. 8.7 noch einmal veranschaulicht ist. Über diese Bereiche gemittelte Größen erhalten im Folgenden den zusätzlichen Index $V$ für das Volumen und $O$ für den Oberflächenbereich. Diese Einteilung bedeutet nicht, dass es nahe der Oberfläche eine andere Phase geben muss, vor allem existiert keine Phasengrenze. Die Größe der Bereiche ist vielmehr so gewählt, dass die Anzahl der darin enthaltenen Ketten eine statistisch signifikante Mittelung erlaubt. Des Weiteren ist anzumerken, dass sich die verschiedenen Bereiche in ihrer Dichte nicht unterscheiden.

Zur Untersuchung der Dynamik wird zum einen die Schwerpunktbewegung der Ketten betrachtet, welche ein Maß für die diffusive Dynamik und Mobilität ist. Zum anderen werden Änderungen im Gyrationsradius als Maß für strukturelle, bzw. konformative Dynamik untersucht. Dabei werden sowohl Simulationszellen hergestellt nach Variante 1 als auch solche, die nach Variante 2 hergestellt worden sind, untersucht. Da die Unterschiede jedoch kleiner als $5 \%$ sind, werden an dieser Stelle nur die Ergebnisse von Variante 2 präsentiert. Ein Vergleich beider Varianten befindet sich im Anhang in Abs. 11.4. 

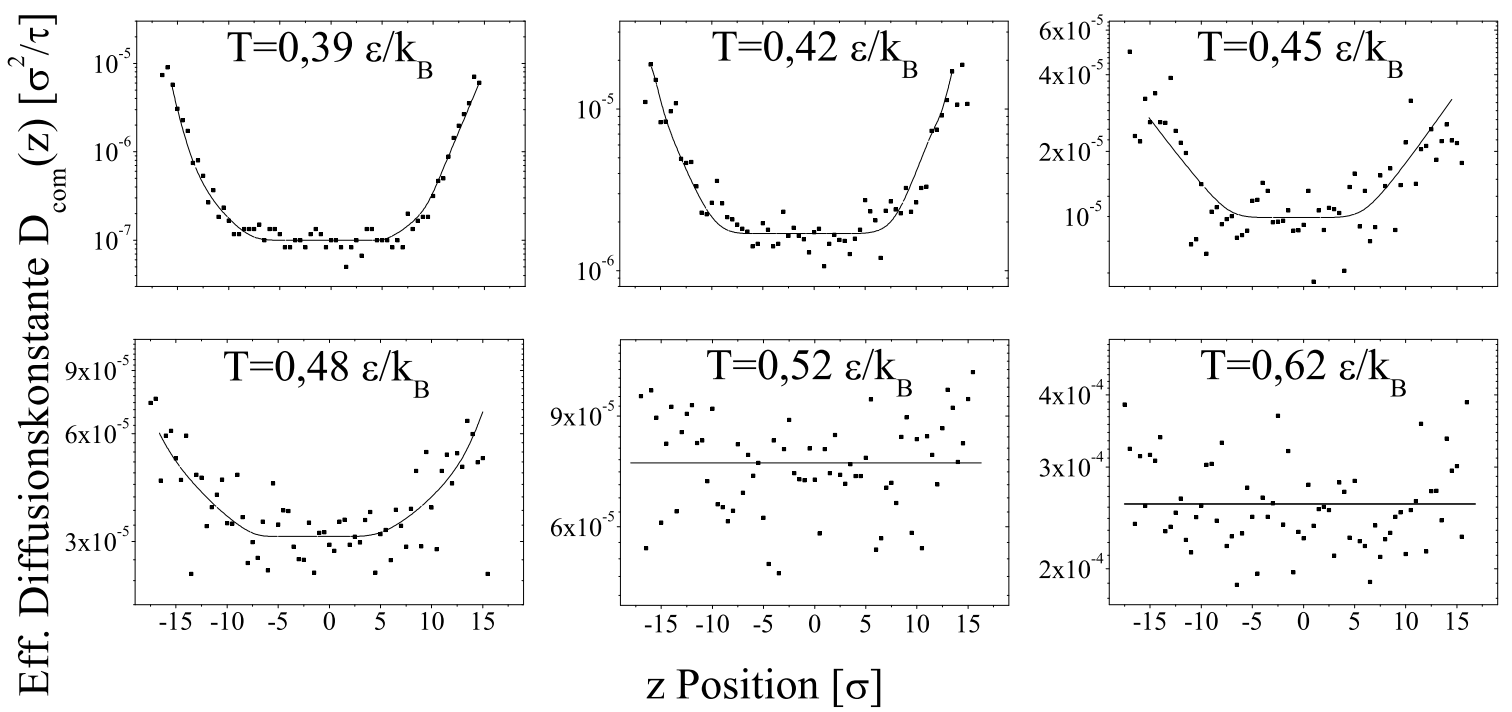

Abbildung 8.8: Tiefenprofile der effektiven Diffusionskonstante $D_{\text {com }}(z)$ auf einer logarithmischen Skala für verschiedene Temperaturen. Die Linien sind Hilfslinien, um den Verlauf zu verdeutlichen. Die freien Oberflächen befinden sich bei $z \approx \pm 15 \sigma$. Die Glasübergangstemperatur beträgt etwa $T_{g} \approx 0,44 \epsilon / \mathrm{k}_{\mathrm{B}}$. Bei niedrigen Temperaturen gibt es zu den Oberflächen hin einen starken Anstieg, der mit zunehmender Temperatur kleiner wird.

\subsubsection{Schwerpunktbewegung}

Wie in Abs. 2.2 beschrieben, kann die Mobilität einer Kette $i$ durch die MSD des Kettenschwerpunkts (vgl. Gl. (2.3)) charakterisiert werden, indem daraus eine effektive Diffusionskonstante $D_{\text {com }, i}$ (vgl. Gl. (2.4)) bestimmt wird. Anschließend werden diese entsprechend ihrer ursprünglichen z-Koordinate gruppiert (hier in Intervalle der Breite $0,5 \sigma)$ und man erhält ein Tiefenprofil der effektiven Diffusionskonstante $D_{\text {com }}(z)$. Solche Tiefenprofile sind in Abb. 8.8 für einige repräsentative Temperaturen aus dem untersuchten Temperaturbereich ${ }^{1}$ gezeigt. Die Spanne reicht von $0,39 \epsilon / \mathrm{k}_{\mathrm{B}}$ - weit unter $T_{g}$, bis $0,62 \epsilon / \mathrm{k}_{\mathrm{B}}$ - weit über $T_{g}$, wobei hier etwa $T_{g} \approx 0,44 \epsilon / \mathrm{k}_{\mathrm{B}}$ gilt.

Mit zunehmender Temperatur steigt die effektive Diffusionskonstante im Volumen $D_{\text {com }, V}$ von $D_{\text {com }, V} \approx 1 \times 10^{-7} \sigma^{2} / \tau$ bei $T=0,39 \epsilon / \mathrm{k}_{\mathrm{B}}$ auf $D_{\text {com }, V} \approx 2,5 \times 10^{-4} \sigma^{2} / \tau$ bei $T=0,62 \epsilon / \mathrm{k}_{\mathrm{B}}$. Diese Werte stimmen mit Simulationen zur Bestimmung der Volumeneigenschaften überein, ${ }^{2}$ die zur Überprüfung ohne Oberflächen, d. h. mit periodischen Randbedingungen in allen Richtungen, durchgeführt worden sind. Zu den Oberflächen hin gibt es bei niedrigen Temperaturen einen starken graduellen Anstieg um nahezu zwei Größenordnungen, der mit steigender Temperatur immer schwächer wird und bei Temperaturen oberhalb von $0,5 \epsilon / \mathrm{k}_{\mathrm{B}}$ kaum noch erkennbar, aber dennoch messbar ist. Das Verhältnis der effektiven Diffusionskonstanten im Volumen $D_{c o m, V}$ und im Ober-

\footnotetext{
${ }^{1}$ Untersucht worden sind 17 verschiedene Temperaturen im Bereich $0,39 \epsilon / \mathrm{k}_{\mathrm{B}} \leq T \leq 0,62 \epsilon / \mathrm{k}_{\mathrm{B}}$.

${ }^{2}$ Somit geben die hier mit Volumen bezeichneten Werte in guter Näherung die Volumeneigenschaften (,Bulk-Eigenschaften“) wieder.
} 


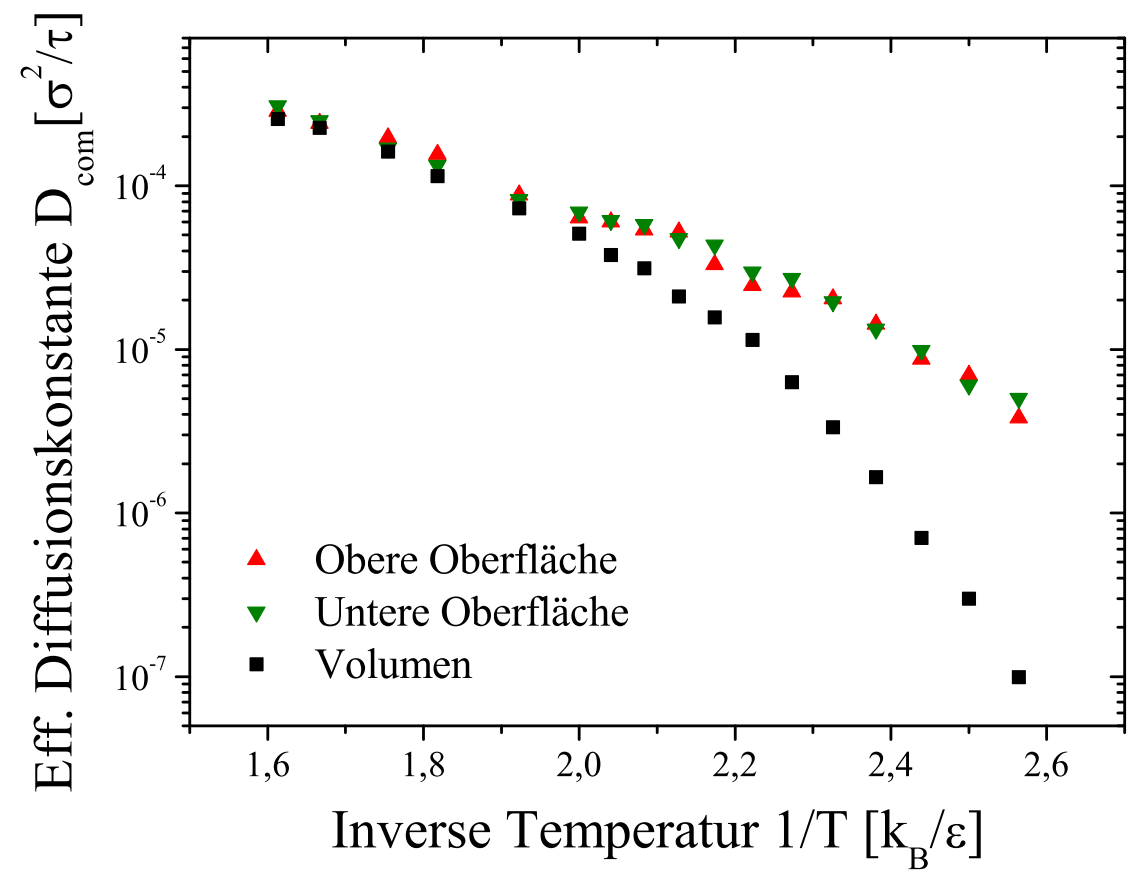

Abbildung 8.9: Arrhenius-Auftragung der effektiven Diffusionskonstante im Volumen (schwarze Quadrate) und in den Oberflächenbereichen (rote (oben) und grüne (unten) Dreiecke). Unterhalb von $T \approx 0,5 \epsilon / \mathrm{k}_{\mathrm{B}}$ werden die Unterschiede zwischen Volumen und Oberfläche deutlich größer.

flächenbereich $D_{\text {com }, O}$ fällt von $\frac{D_{c o m, O}}{D_{c o m, V}} \approx 45$ bei $T=0,39 \epsilon / \mathrm{k}_{\mathrm{B}}$ auf $\frac{D_{\text {com }, O}}{D_{c o m, V}} \approx 1,3$ für $T \geq 0,5 \epsilon / \mathrm{k}_{\mathrm{B}}$.

Um dieses Verhalten weiter zu quantifizieren, ist in Abb. 8.9 eine Arrhenius-Auftragung der effektiven Diffusionskonstante für das Volumen und für die Oberflächenbereiche gezeigt. Hier erkennt man klar, dass der Unterschied zwischen Volumen und Oberfläche unterhalb von $T \approx 0,5 \epsilon / \mathrm{k}_{\mathrm{B}}$ mit abnehmender Temperatur deutlich größer wird. Aber auch darüber ist der Wert für Oberflächendiffusion größer als für Volumendiffusion.

\subsubsection{Fluktuationen des Gyrationsradius}

Der Gyrationsradius ist ein Maß für die Größe und die räumliche Ausdehnung einer Kette und somit bedingen dessen Änderungen auch Änderungen in der Konformation der Kette. Daher werden hier zeitliche Fluktuationen von $R_{G}^{2}$ als Maß für die konformative Dynamik betrachtet. Dafür werden für jede Kette $i$ die Fluktuationen im Zeitraum $\left[t_{0} . . t\right]$ (im Folgenden nur durch $t$ angegeben) wie in Abs. 2.2 in Gl. (2.6) beschrieben bestimmt. Anschließend werden diese Fluktuationen $\Delta R_{G, i}^{2}(t)$ entsprechend der ursprünglichen z Position des Kettenschwerpunkts gruppiert und gemittelt. Auf diese Weise erhält man Tiefenprofile für die Fluktuationsstärke ähnlich zu denen der effektiven Diffusionskonstante in Abb. 8.8. Da bei der Berechnung der Fluktuationen die Länge des Beobachtungszeitraums eine Rolle spielt, können zwei verschiedene Einflüsse 


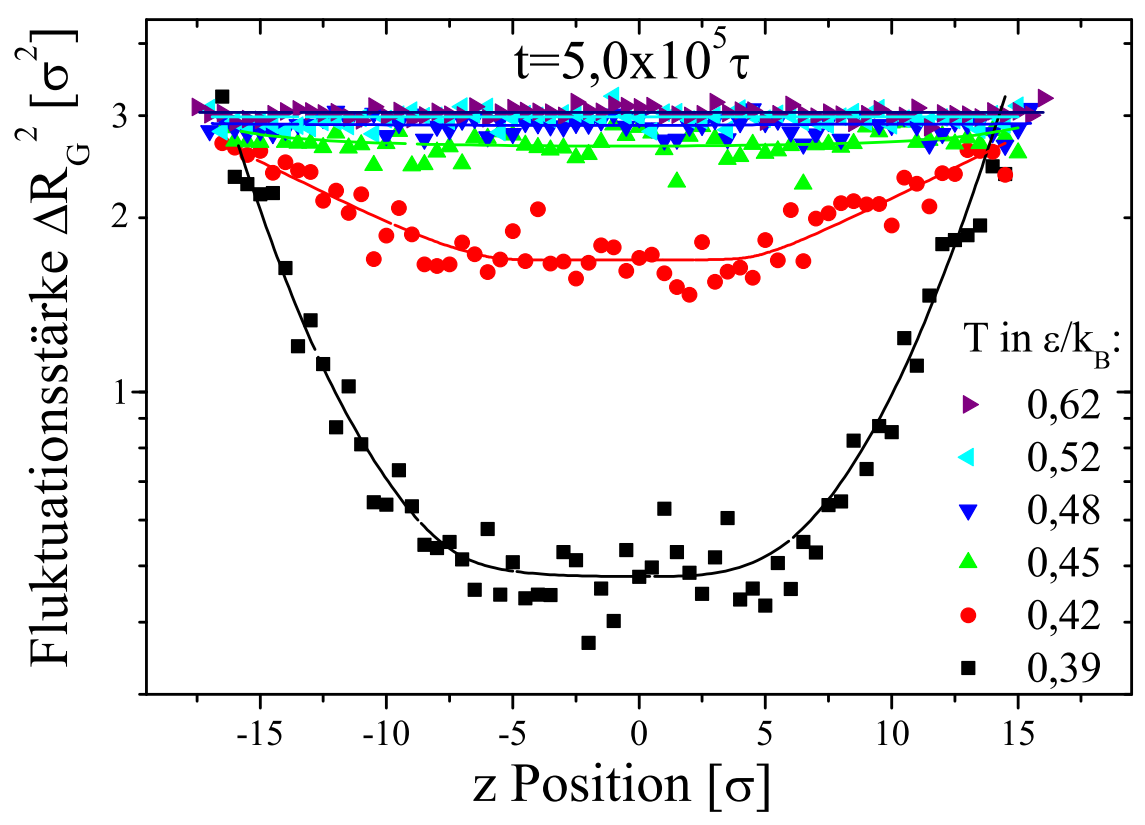

Abbildung 8.10: Tiefenprofil der Fluktuationsstärke des Gyrationsradius bei festem Beobachtungszeitraum der Länge $t=5 \times 10^{5} \tau$ für verschiedene Temperaturen. Die Linien sollen den Verlauf der Kurven verdeutlichen. Bei niedrigen Temperaturen ist ein ausgeprägter Anstieg zu den Oberflächen hin zu erkennen, der bei höheren Temperaturen verschwindet.

untersucht werden, zum einen der Einfluss der Temperatur bei gleicher Beobachtungszeit und zum anderen die Länge der Beobachtungszeit bei konstanter Temperatur.

Abb. 8.10 zeigt einige repräsentative Tiefenprofile für verschiedene Temperaturen mit fester Beobachtungszeit $t=5 \times 10^{5} \tau$. Bei niedrigen Temperaturen gibt es zu den Oberflächen hin einen starken Anstieg der Fluktuationsstärke. Dieser Anstieg wird mit steigender Temperatur immer weniger ausgeprägt und verschwindet bei dieser Beobachtungszeit oberhalb von $T=0,45 \epsilon / \mathrm{k}_{\mathrm{B}}$. Im Volumen wächst die Fluktuationsstärke mit der Temperatur bis zu einem maximalen Wert von $\Delta R_{G}^{2} \approx 3 \sigma^{2}$ an. Für $T \geq 0,48 \epsilon / \mathrm{k}_{\mathrm{B}}$ liegen alle Kurven in der Nähe dieses Maximalwerts.

Die Veränderung der Tiefenprofile der Fluktuationsstärke mit der Länge des Beobachtungszeitraums ist in Abb. 8.11 für $T=0,45 \epsilon / \mathrm{k}_{\mathrm{B}}$ gezeigt. Der Verlauf der Kurven ist ähnlich wie der für steigende Temperaturen, bei kurzen Zeiten ist der Anstieg zu den Oberflächen stärker ausgeprägt und mit zunehmender Zeit wird zum einen der Anstieg geringer und zum anderen steigt die Fluktuationsstärke und nähert sich dem Wert $\Delta R_{G}^{2} \approx 3 \sigma^{2}$ an. Ein Unterschied zwischen Volumen und Oberflächen ist also nur bei niedrigen Temperaturen, bzw. kurzen Beobachtungszeiten zu erkennen. Je höher die Temperatur, desto eher ist in der Fluktuationsstärke kein Unterschied mehr erkennbar. Der Übergang zwischen Bereichen mit erkennbaren und verschwindenden Unterschieden scheint dabei sowohl für wachsende Beobachtungszeiten als auch für steigende Temperaturen glatt zu sein.

Um den Einfluss der Oberflächen weiter zu untersuchen, ist in Abb. 8.12 das An- 


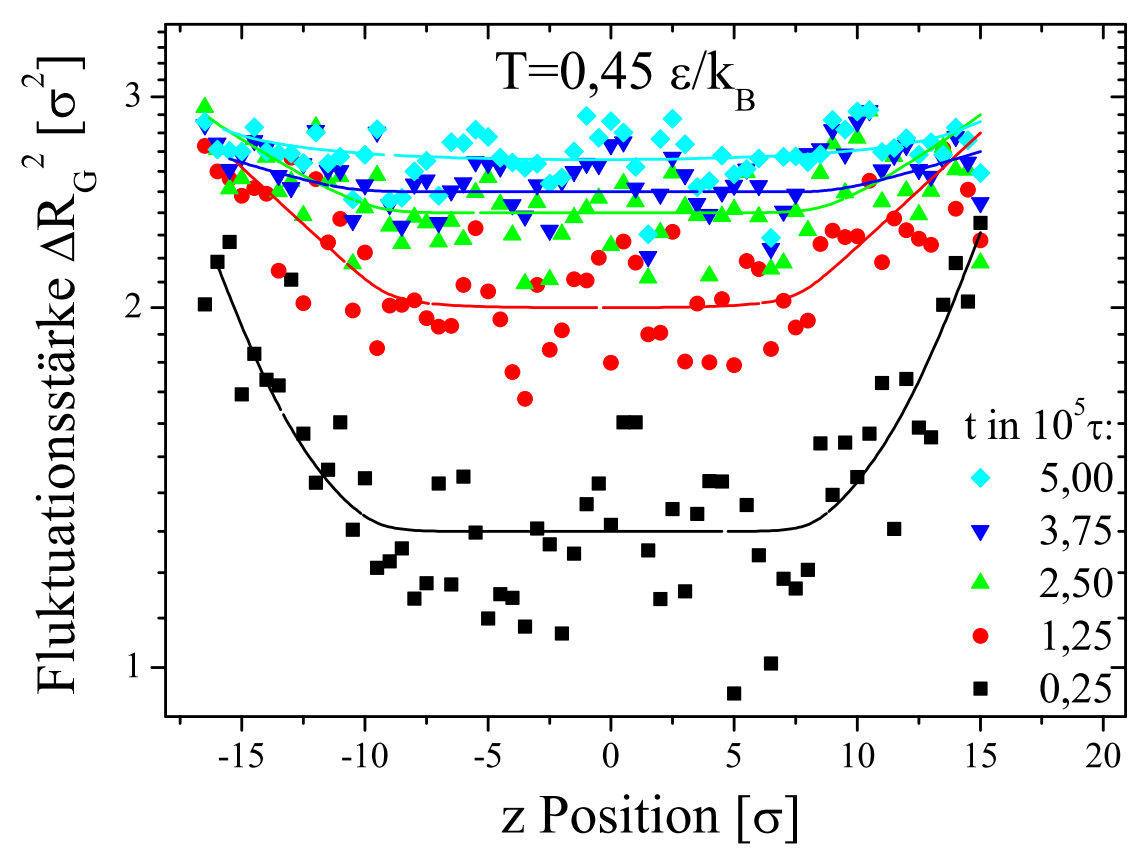

Abbildung 8.11: Tiefenprofil der Fluktuationsstärke des Gyrationsradius bei konstanter Temperatur $T=0,45 \epsilon / \mathrm{k}_{\mathrm{B}}$ für verschiedene Beobachtungszeiträume. Die Linien sollen den Verlauf der Kurven verdeutlichen. Betrachtet man das System nur für kurze Zeit, erkennt man deutliche Unterschiede zwischen Volumen und Oberflächen, die nach längerer Betrachtungszeit verschwinden.

wachsen der mittleren Fluktuationsstärke mit der Länge des Beobachtungszeitraums für verschiedene Temperaturen sowohl für das Volumen (volle Symbole) als auch für den Oberflächenbereich (offene Symbole) aufgetragen. Alle Kurven steigen mit der Zeit an, wobei der Anstieg für höhere Temperaturen schneller erfolgt. Bei niedrigen Temperaturen $\left(T<0,45 \epsilon / \mathrm{k}_{\mathrm{B}}\right)$ ist für alle betrachteten Zeiträume ein deutlicher Unterschied zwischen Volumen und Oberfläche zu erkennen, der bei hohen Temperaturen $\left(T>0,45 \epsilon / \mathrm{k}_{\mathrm{B}}\right)$ selbst bei kurzen Zeiten schwer zu erkennen ist. Im Temperaturbereich dazwischen verschwindet der Unterschied für längere Beobachtungszeiträume.

Man kann dieselbe Art der Auswertung auch für den End-zu-End Abstand durchführen, was zu qualitativ gleichen Ergebnissen führt. 


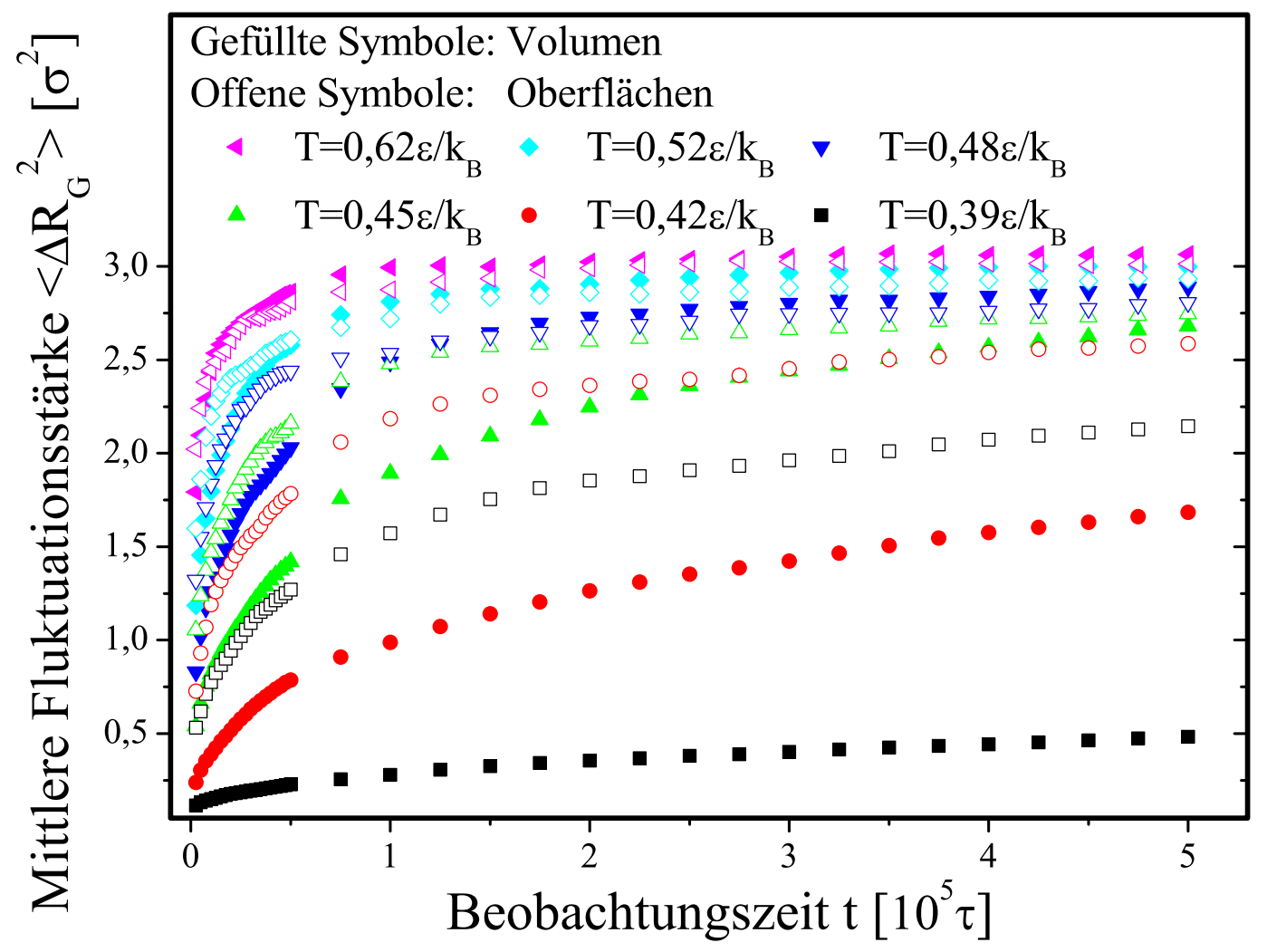

Abbildung 8.12: Entwicklung der Fluktuationsstärke des Gyrationsradius mit der Länge des Beobachtungszeitraums für verschiedene Temperaturen. Alle Kurven steigen mit der Zeit an, wobei der Anstieg mit steigender Temperatur schneller erfolgt. 


\section{Diskussion}

In diesem Kapitel sollen die Ergebnisse der vorangegangenen Kapitel diskutiert werden. Zunächst wird die Struktur der thermisch aufgedampften PC Schichten behandelt, bevor die Topographieentwicklungen betrachtet werden. Dabei erfolgt ein Vergleich der experimentellen Ergebnisse mit den Kontinuumsmodellierungen. Anschließend wird die Dynamik von dünnen Polymerfilmen anhand der MD Simulationsergebnisse diskutiert.

\subsection{Struktur der thermisch aufgedampften PC Schichten}

Ein wichtiger Punkt bei der Untersuchung des thermischen Aufdampfens von Polymeren ist die Frage nach der Veränderung der Struktur und des Aufbaus des Polymers. Durch die relativ hohen Temperaturen beim Aufdampfen kann es neben der Überführung in die Gasphase bei Polymeren (und anderen molekularen Systemen) noch zu weiteren Prozessen, wie etwa Vernetzung oder auch Zersetzung, kommen. Es gibt eine Reihe von zersetzenden Prozessen, die bei verschiedenen Temperaturen stattfinden. So können bei der Degradation einzelne Bindungen irgendwo entlang einer Kette ${ }^{1}$ aufgebrochen werden, wodurch sie in zwei kürzere Ketten zerbricht und eine große Vielzahl unterschiedlicher Kettenlängen entsteht. Wird eine Kette nicht irgendwo, sondern an den Enden aufgebrochen, spricht man von Depolymerisation, da das Polymer in seine monomeren Bestandteile zerlegt wird. Beide Prozesse verringern das Molekulargewicht, der kettenartige Charakter der Polymere bleibt aber über einen längeren Zeitraum erhalten [Kub94]. Bei der Dekomposition schließlich wird das gesamte Molekül schnell zersetzt. Alle diese Prozesse sind durch die Temperatur aktiviert und können somit durch Dissoziationsenergien charakterisiert werden. Die vollständige Dekomposition muss beim Aufdampfen vermieden werden, wenn der polymere Charakter erhalten bleiben soll. Die Temperatur muss dementsprechend niedrig gehalten werden.

\subsubsection{Thermische Zersetzung von PC}

Die thermische Zersetzung anderer Polymere wie Polyethylen und Polystyrol wurde bereits seit Ende der 40'er Jahre von Jellinek [Jel48; Jel49a; Jel49b] und Madorsky [Mad48; Mad52; Mad53] intensiv untersucht. Dabei lag das Hauptaugenmerk auf dem Gewicht und der Viskosität des zurückbleibenden Materials, während die abdampfenden Bestandteile nur in wenigen Studien untersucht, bzw. nur zum Nachweis der Zersetzung detektiert wurden. In diesen Arbeiten wurden im Vakuum Temperaturen zwischen $240{ }^{\circ} \mathrm{C}$ und $420^{\circ} \mathrm{C}$ untersucht. Polyethylen zerfällt über $350^{\circ} \mathrm{C}$ in kürzere Ketten mit

\footnotetext{
${ }^{1}$ Entlang der Kette gibt es statistisch verteilte schwächere Bindungen.
} 
einem Molekulargewicht um $M=2300 \mathrm{~g} / \mathrm{mol}$, die abdampfen können, unterhalb von $350{ }^{\circ} \mathrm{C}$ verdampfen nur Ketten mit maximal 23 Kohlenstoffatomen [Kub94]. Polystyrol depolymerisiert bei Temperaturen zwischen $325^{\circ} \mathrm{C}$ und $375^{\circ} \mathrm{C}$ im Vakuum [Mad52]. Über $400^{\circ} \mathrm{C}$ kommt es zur Dekomposition [Mad48]. Es gibt auch einige Studien zum thermischen Aufdampfen von Polyethylen [Luf70; Mak90; Ued98]. Dabei zeigte sich, dass ein Problem bei der Herstellung die starke Blasenbildung im Tiegel (sieden) ist, da dabei größere Mengen Material auf das Substrat spritzen können. Außerdem neigen die Schichten zur Kristallisation.

Ähnliche Untersuchungen zur thermischen Zersetzung sind in den letzten 50 Jahren auch an Polycarbonaten durchgeführt worden, wobei auch dort hauptsächlich das zurückbleibende Material untersucht wurde [Lee64; Dav64; Gol64; Dav68; McN91; McN93; Mon87; Mon92]. Trotz der vielen verschiedenen Untersuchungen sind die genauen Mechanismen der Zersetzung immer noch nicht im Detail verstanden (eine Übersicht über verschiedene Arbeiten ist in [Rob01] zu finden). Außerdem gibt es große Unterschiede je nachdem, ob die Zersetzung unter Stickstoffatmosphäre, an Luft oder im Vakuum untersucht wird, wie Thermogravimetriemessungen von Li und Huang zeigen [Li99]. Dabei liegt die charakteristische Zersetzungstemperatur im Vakuum bei $548^{\circ} \mathrm{C}$ deutlich höher als an Luft $\left(458^{\circ} \mathrm{C}\right)$. Nach Davis und Golden [Dav64; Gol64; Dav68], die Viskositätsmessungen, Massenspektrometrie und Gaschromatographie zur Untersuchung der Zersetzung im Vakuum verwendet haben, ist Degradation, also ein Spalten der Ketten an statistisch entlang der Hauptkette verteilten schwächeren Bindungen, der dominante Zersetzungsprozess. Unterhalb von $400{ }^{\circ} \mathrm{C}$ findet diese Spaltung überwiegend an den Carbonatgruppen statt, darüber an den Isopropylidengruppen [Li99; Rob01]. In Abb. 9.1 (aus [Dav64]) ist das Molekulargewicht von PC in Abhängigkeit von der Haltezeit bei einer bestimmten Temperatur im Vakuum dargestellt. Unterhalb von $260{ }^{\circ} \mathrm{C}$ findet kaum Zersetzung statt. Bei $305^{\circ} \mathrm{C}$ halbiert sich das Molekulargewicht nach etwa 200 h. Bei höheren Temperaturen steigt die Zersetzungsrate stark an. Die Anzahl der schwächeren Bindungen $N$ nimmt bei konstanter Temperatur mit der Zeit ab und folgt dabei einer Kinetik 1. Ordnung, d. h. es gilt: $N(t)=N_{0} e^{-k t}$, wobei $k$ eine Ratenkonstante ist. Untersucht man PC dagegen bei $380^{\circ} \mathrm{C}$ an Luft, bildet sich durch Quervernetzung ein spröder, nicht löslicher und schwarzer Rest [Dav64]. Nach Lee startet die Zersetzung von PC oberhalb von $310^{\circ} \mathrm{C}$ mit einem Oxidationsschritt [Lee64]. Andere Gruppen haben als Hauptdegradationsprodukt cyclische Oligomere gefunden [McN91; McN93; Mon87; Mon92], wobei auch hier der initiale Schritt kontrovers diskutiert wird. Bei höheren Temperaturen können zudem noch Vernetzung $\left(\mathrm{ab} 350{ }^{\circ} \mathrm{C}\right.$ [Yak61]) und Depolymerisation (ab $340^{\circ} \mathrm{C}$ [Lee64]) wichtig werden. Dieser Überblick zeigt bereits, dass die Zersetzung von PC noch nicht im Detail verstanden ist. Die Ergebnisse hängen stark von den Untersuchungsmethoden, dem verwendeten Polycarbonat, dem Temperaturbereich und den Umgebungsbedingungen ab.

Im Gegensatz zu den meisten der oben erwähnten Studien wird in dieser Arbeit nur der verdampfende Anteil untersucht. Die gute qualitative Übereinstimmung der FTIR Messungen an thermisch aufgedampften PC und Daten von PC Massivproben aus der Literatur (vgl. Abb. 6.2) legt nahe, dass der Verdampfungsvorgang die zugrundeliegende Bindungsstruktur nicht, bzw. nur wenig ändert. Dekomposition der Moleküle kann somit ausgeschlossen werden. Die Abnahme der Intensität im Bereich $1000 \mathrm{~cm}^{-1} \leq \nu \leq 1200 \mathrm{~cm}^{-1}$ und $2850 \mathrm{~cm}^{-1} \leq \nu \leq 2960 \mathrm{~cm}^{-1}$ (C-H Bindung) sowie 


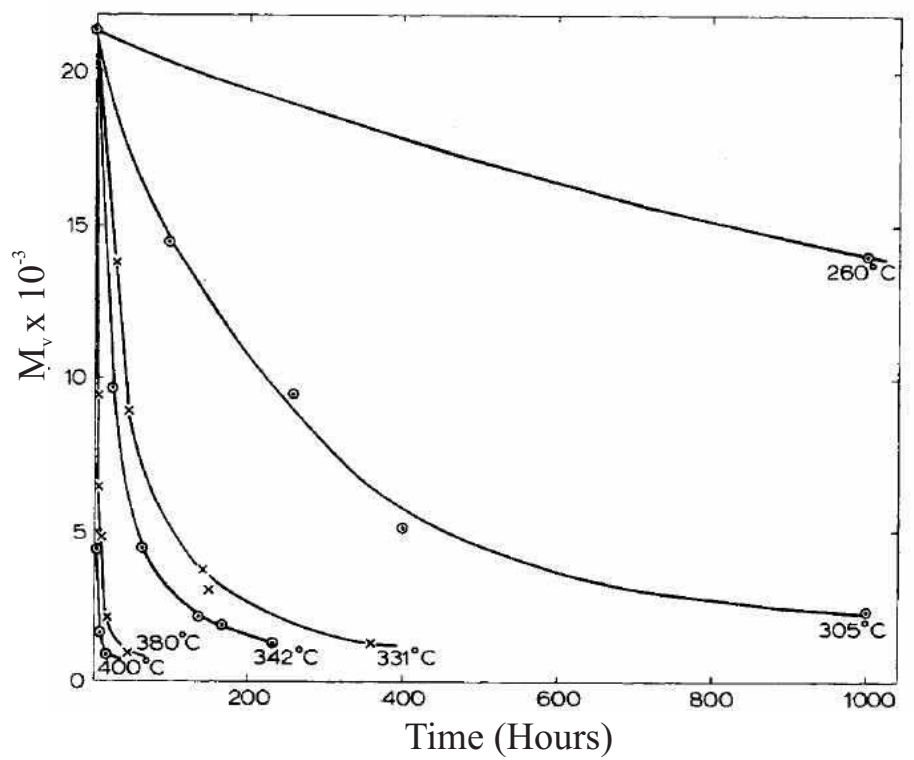

Abbildung 9.1: Einfluss der Haltezeit bei verschiedenen Temperaturen im Vakuum auf das Molekulargewicht von PC nach Golden und Davis (aus [Dav64]). Unterhalb von $260{ }^{\circ} \mathrm{C}$ findet nahezu keine Zersetzung statt. Auch bei $300{ }^{\circ} \mathrm{C}$ sollte die Zersetzung in den ersten $200 \mathrm{~h}$ gering sein.

der $\mathrm{C}=\mathrm{C}$ und $\mathrm{C}-\mathrm{O}$ Bindungen (vgl. Tab. 6.1) und die Zunahme bei $\nu \gtrsim 3200 \mathrm{~cm}^{-1}$ (Phenol, O-H) sind Hinweise auf eine verkürzte Kettenlänge, da bei kürzeren Ketten mehr Kettenenden vorhanden sind und mehr endständiges Phenol auftreten kann. Dadurch steigt der Einfluss der Enden im Vergleich zur Gesamtkette. Dass die Herstellungsmethode noch größeren Einfluss haben kann, zeigen Vergleiche zu PC Filmen, die mit gepulster Laserdeposition (PLD) hergestellt worden sind [Vre05]. Abb. 9.2 zeigt neben den bereits vorgestellten FTIR Messungen aus der Literatur und an thermisch aufgedampftem PC Messungen an laserdeponiertem PC. Auch bei der verwendeten niedrigen mittleren Energiedichte ist der Energieeintrag bei der PLD größer als beim thermischen Verdampfen und es werden mehr Bindungen gebrochen, was zu deutlich stärkeren Veränderungen führt. Bei höherer Energiedichte zeigen die FTIR Messungen kein Signal mehr [Hac04].

Die Hinweise auf eine Verkürzung der Kettenlänge werden durch die Größenausschlusschromatographie (SEC) Messungen bestätigt (vgl. Abb. 6.3), obwohl die Reduzierung der Molmasse bei $300{ }^{\circ} \mathrm{C}$ selbst bei einer Verdampfungszeit von $96 \mathrm{~h}$, die für die Herstellung der SEC Proben benötigt werden, ${ }^{2}$ gering sein sollte (vgl. Abb. 9.1 [Dav64]). Diese zeigen eine deutliche Verschiebung des Signals zu kleinen Molmassen hin und nur sehr wenig Intensität bei der Ausgangsmolmasse. Zusammen mit den Ergebnissen der Massenspektrometrie (vgl. Abb. 6.4 und 11.1) ergibt sich, dass die thermisch aufgedampften Filme aus oligomeren Polycarbonat bestehen, also aus Bruchstücken der ursprünglichen Kette mit einer Länge von ca. $2-7$ Monomeren. Dabei treten neben reinen Oligomeren, also Vielfachen des Monomers - wie z. B. das Trimer

\footnotetext{
${ }^{2}$ Die effektive Zeit für das Material im Tiegel bei $300{ }^{\circ} \mathrm{C}$ beträgt sogar nur $48 \mathrm{~h}$, da der Tiegel einmal nachgefüllt werden muss.
} 


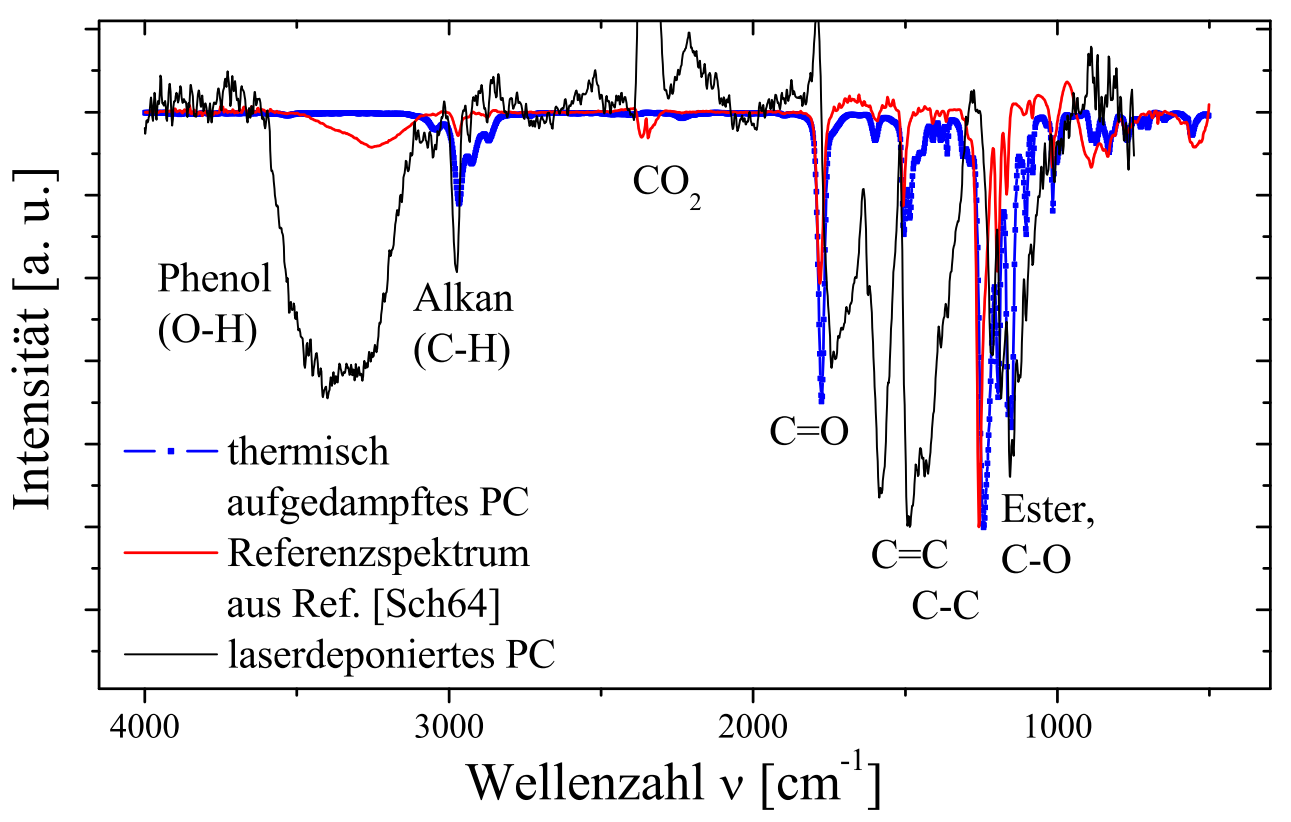

Abbildung 9.2: Vergleich von FTIR Messungen aus der Literatur [Sch64], an thermisch aufgedampftem PC und an laserdeponiertem PC [Vre05]. Im Vergleich zum thermisch aufgedampften PC weisen die laserdeponierten Schichten deutlich stärkere Abweichungen vom Literaturspektrum auf.

bei $m / z=780 \mathrm{u}$, auch Bruchstücke mit dazwischenliegender Masse auf. Z. B. gehört $m / z=1248 \mathrm{u} \mathrm{zu} \mathrm{C}_{77} \mathrm{H}_{65} \mathrm{O}_{15}$, was einem Fragment aus einem Pentamer, dem ein Propylen Baustein $\left(\mathrm{C}_{3} \mathrm{H}_{6}\right)$ fehlt, entspricht. In Abb. $11.1 \mathrm{im}$ Anhang ist die Strukturformel eines solchen Fragments eingezeichnet. Solche Stücke entstehen durch Spaltung innerhalb eines Monomers in der Kette. Da die Massenspektrometrie nur Masse-zu-Ladungs Verhältnisse von bis zu $m / z=2000 \mathrm{u}$ detektieren kann, ist nicht auszuschließen, dass die PC Schichten auch höhere Molmassen enthalten. Auch über den Anteil und das tatsächliche Gewicht des SEC Signals bei hohen Molmassen kann keine genaue Aussage getroffen werden, der Anteil ist aber klein.

Es ist festzuhalten, dass die thermisch aufgedampften PC Schichten hauptsächlich aus oligomeren Polycarbonat bestehen und es keine Hinweise auf Dekomposition oder Vernetzung gibt.

\subsubsection{Thermisches Aufdampfen von PC}

Beim thermischen Aufdampfen muss die Verdampfungsenthalpie $\Delta H$ aufgebracht werden, um das Material vom flüssigen in den gasförmigen Zustand zu überführen. Bei Polymeren ist diese Verdampfungsenthalpie durch die Größe der Makromoleküle und damit zusammenhängende Verschlaufungseffekte und eine erhöhte Anzahl von Bindungen zu benachbarten Teilchen im Vergleich zu kurzkettigen oder atomaren Bausteinen erhöht. Die zur Bildung einer Gasphase aus den ganzen Makromolekülen benötigte Temperatur liegt dementsprechend hoch, so dass bereits thermische Zersetzung stattfinden kann. Durch die Zersetzung werden die Ketten kürzer, solange keine Dekomposition 
stattfindet und die Ketten vollständig zerfallen. Für diese kürzeren Anteile ist die Verdampfungsenthalpie niedriger und sie verdampfen mit einer höheren Wahrscheinlichkeit, da die Verdampfungswahrscheinlichkeit durch einen Boltzmann-Faktor $e^{-\Delta H / k_{B} T}$ gegeben ist. D. h. je kürzer die Ketten werden, desto eher verdampfen sie aus dem Tiegel und können auf dem Substrat kondensieren. Somit ist auch klar, dass der Anteil an Material mit hoher Molmasse bei thermisch aufgedampften PC Schichten sehr klein ist (vgl. SEC Messungen in Abb. 6.3).

Bevor also beim thermischen Aufdampfen Material in die Gasphase übergehen kann, müssen kleinere Fragmente erzeugt werden, die bei der eingestellten Temperatur verdampfen können. Diese Fragmentierung erfolgt durch Degradation, die an statistisch entlang der Hauptketten verteilter schwächerer Bindungen ansetzt. Die Abnahme der Anzahl dieser spaltbaren Bindungen folgt nach Golden und Davis, wie oben beschrieben, einer Kinetik 1. Ordnung mit einer Ratenkonstante $k$ [Dav64]. D. h. die Erzeugung von kurzkettigen Fragmenten, die verdampfen können, wird mit zunehmender Zeit langsamer und in gleicher Weise sollte für längere Zeiten, wenn die anfänglich vorhandenen kurzkettigen Fragmente verdampft sind und die Erzeugung der Bruchstücke der ratenbegrenzende Prozess ist, auch die Depositionsrate $\nu$ abnehmen:

$$
\nu(t)=\nu_{0} e^{-k t}
$$

Wie bereits in Abs. 3.1.2 erwähnt, ist während des Aufdampfvorgangs eine Abnahme der Depositionsrate von etwa $3 \AA /$ min zu Beginn auf etwa $1 \AA /$ min zu beobachten. In Abb. 9.3 ist ein typischer zeitlicher Verlauf der Depositionsrate, bzw. des Logarithmus der Depositionsrate, während des Aufdampfens aufgetragen (blaue Quadrate). Zusätzlich ist eine Anpassung gemäß des exponentiellen Abfalls aus Gl. (9.1) mit den Parametern $\nu_{0} \approx 0,03 \AA \mathrm{s}^{-1}$ und $k \approx 1,5 \times 10^{-5} \mathrm{~s}^{-1}$ eingezeichnet (blaue Linie), die die Annahme, dass die Kinetik 1. Ordnung ist, bestätigt. Dieser Wert von $k$ ist deutlich höher als Werte von Davis und Golden (extrapoliert zu $300{ }^{\circ} \mathrm{C}: k \approx 5 \times 10^{-8} \mathrm{~s}^{-1}$ ) [Dav64], aber vergleichbar mit denen von Robertson und Ward (extrapoliert zu $300{ }^{\circ} \mathrm{C}$ : $k \approx 7 \times 10^{-6} \mathrm{~s}^{-1}$ ) [Rob02a], die das gleiche Polycarbonat wie in dieser Arbeit untersucht haben. Die Reaktionsgeschwindigkeit hängt demnach vom genauen Aufbau des Polycarbonats ab, während die Mechanismen die gleichen sind.

Unter der Annahme, dass zu Beginn des Aufdampfvorgangs genügend kurzkettige Fragmente vorhanden sind, bzw. diese schnell genug erzeugt werden können, so dass die Rate nicht durch den Erzeugungsschritt begrenzt ist, kann aus der Temperaturabhängigkeit der anfänglichen Depositionsrate die Verdampfungsenthalpie bestimmt werden. Dazu sind Schichten bei $290{ }^{\circ} \mathrm{C}, 300{ }^{\circ} \mathrm{C}$ und $310{ }^{\circ} \mathrm{C}$ hergestellt worden. In Abb. 9.3 ist der Logarithmus der Depositionsrate gegen den Kehrwert der Temperatur aufgetragen (schwarze Sterne) und durch eine Anpassung (schwarze Linie) gemäß $\nu(T)=A e^{\frac{-\Delta H}{k_{B} T}}$ wird die Verdampfungsenthalpie zu $\Delta H \approx 125 \mathrm{~kJ} / \mathrm{mol}$ bestimmt. Dieser Wert ist deutlich niedriger als Werte für die Aktivierungsenergie der Zersetzung von $\mathrm{PC}$, für die es in der Literatur verschiedene Angaben gibt: Golden und Davis geben $E_{a} \approx 165 \mathrm{~kJ} / \mathrm{mol}$ [Dav64] an, bei Robertson und Ward findet man $E_{a} \approx 190 \mathrm{~kJ} / \mathrm{mol}$ [Rob02a] und bei Knight $E_{a} \approx 149 \mathrm{~kJ} / \mathrm{mol}$ [Kni78]. Die Zersetzung ist zu Beginn also nicht der ratenbegrenzende Prozess und die Annahmen zur Bestimmung der Verdampfungsenthalpie sind bestätigt. 


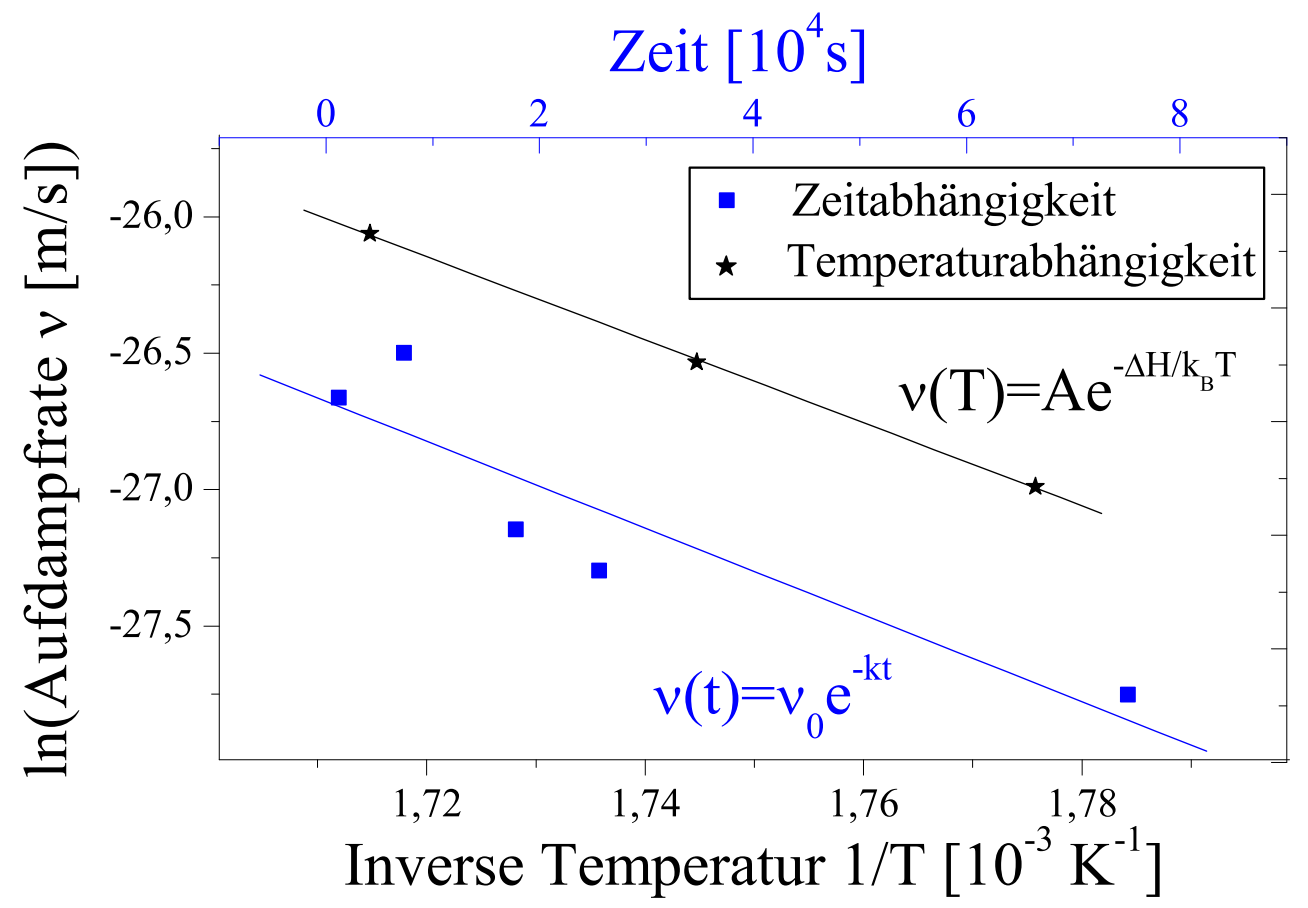

Abbildung 9.3: Abhängigkeit der (logarithmischen) Depositionsrate von der Aufdampfzeit bei $300{ }^{\circ} \mathrm{C}$ (blaue Quadrate, obere Abszisse) mit einer Anpassung (blaue Linie) gemäß $\nu(t)=\nu_{0} e^{-k t}$ (Gl. (9.1)) mit den Parametern $\nu_{0} \approx 0,03 \AA_{\mathrm{s}}^{-1}$ und $k \approx 1,5 \times 10^{-5} \mathrm{~s}^{-1}$ und von der inversen Temperatur (schwarze Sterne, untere Abszisse) mit einer Anpassung (schwarze Linie) gemäß $\nu(T)=A e^{\frac{-\Delta H}{k_{B} T}} \operatorname{mit} \Delta H \approx 125 \mathrm{~kJ} / \mathrm{mol}$.

Zusammenfassend heißt das, dass beim thermischen Aufdampfen von PC hauptsächlich kurzkettiges Material verdampft wird, das erst durch thermische Degradation gebildet werden muss. Zu Beginn sind genügend kurze Ketten vorhanden, bzw. sie werden schnell genug erzeugt, um den Verdampfungsprozess und damit die Depositionsrate nicht zu begrenzen. Mit zunehmender Zeit wird die Depositionsrate dann durch die Erzeugung von kürzeren Ketten begrenzt, die sich aufgrund der abnehmenden Zahl der schwächeren Bindungen verlangsamt.

Die erzeugten Schichten sind amorph, wie das breite Maximum der Weitwinkelröntgenmessung zeigt (vgl. Abb. 6.1). Es gibt im Gegensatz zu thermisch aufgedampften Polystyrol Schichten (vgl. Abb. 11.3) und Polyethylen Schichten [Luf70; Ued98] keine Anzeichen von Kristallisation. Der Vergleich mit Weitwinkelmessungen an Massivproben aus der Literatur [Nek73] zeigt ähnliche Form und Breite der Maxima. Dies ist ein weiterer Hinweis darauf, dass beim thermischen Aufdampfen die chemische Struktur des PC erhalten bleibt und sich nur die Kettenlänge verkürzt. Die Lage der Grundbausteine zueinander ist dadurch nur wenig beeinflusst. 


\subsection{Topographieentwicklung}

Nachdem Struktur und chemische Zusammensetzung der thermisch aufgedampften PC Schichten charakterisiert sind, wird im Folgenden ihre Topographie behandelt. Dabei stehen zunächst die Anfangsstadien und der Einfluss der verschiedenen Substrate im Vordergrund. In diesem Zusammenhang werden auch die Ergebnisse der Kontinuumsmodellierung diskutiert und mit den experimentellen Ergebnissen verglichen. Anschließend wird noch die Strukturentwicklung der dünnen Schichten auf anorganischen Substraten mit der Zeit behandelt.

\subsubsection{Einfluss der Grenzflächen auf die Anfangsstadien beim thermischen Aufdampfen}

Die Topographien von dünnen thermisch aufgedampften PC Schichten auf $\mathrm{Si}, \mathrm{SiO}_{2}$ und GaAs weisen eine ausgeprägte Struktur mit graben- oder lochartigen Vertiefungen auf. Auf GaAs sind diese eher länglich und auf $\mathrm{SiO}_{2}$ eher rund, während auf Si eine Mischung aus beiden zu erkennen ist. Diese Vertiefungen reichen bis zum Substrat, wie man aus den zugehörigen Phasenbildern, die hier nicht gezeigt sind, erkennen kann. Der Phasenkontrast, also die Verschiebung zwischen Anregungssignal und Antwort des Cantilevers, in TappingMode ${ }^{T M}$ AFM Messungen beinhaltet Informationen über die Wechselwirkungen zwischen Spitze und Probe und bietet so die Möglichkeit, Materialunterschiede festzustellen [Tam96]. Allerdings kann eine sehr dünne, wenige nm dicke, zurückbleibende Lage aus PC im Phasenkontrast nicht gesehen werden, so dass eine solche nicht auszuschließen ist. Die Strukturen weisen eine große Ähnlichkeit zu Strukturen von entnetzenden Polystyrol Filmen auf Si Substraten mit 190 nm dicker Oxidschicht [See01b; See01a] und ohne Oxidschicht auf [Xie98].

Entnetzung ist ein bei Flüssigkeiten und Polymeren nahe ihrer Glasübergangstemperatur bekanntes Phänomen [Gen85; Rei01; See01b; See01a; Bec03], das aber auch bei metallischen Schichten beobachtet wird [Pet08]. Dabei ist ein dünner Film auf einer für ihn nicht benetzbaren Oberfläche nicht stabil sondern entnetzt, zieht sich also zu Tropfen zusammen. Entnetzung kann an Defekten starten oder durch thermische Fluktuationen, die exponentiell anwachsen können, initiiert werden (spinodale Entnetzung). Wesentlich für die Benetzbarkeit ist die Wechselwirkung zwischen Material und Substrat, die durch das in Abs. 4.2.1 eingeführte effektive Grenzflächenpotential $\Phi(h)=\frac{\epsilon}{(h+d)^{8}}-\frac{A}{12 \pi(h+d)^{2}}$ beschrieben werden kann [Isr92], das die freie Energie der Wechselwirkung pro Einheitsfläche beschreibt [Vri66]. Der Beitrag der langreichweitigen van-der-Waals Kräfte, der durch die effektive Hamaker-Konstante $A$ gegeben ist, ist entscheidend dafür, ob ein System be- oder entnetzend ist. Da ein System im Gleichgewicht ist, wenn die freie Energie minimal wird, liefert das globale Minimum des effektiven Grenzflächenpotentials die Gleichgewichtsfilmdicke [Mit93; See01a]. Somit bildet sich ein homogener Film, wenn das globale Minimum bei $h=\infty$ liegt, was nur für $A<0$ und $\Phi^{\prime \prime}>0$ möglich ist. Dann ist das System vollständig benetzend und wird stabil genannt (vgl. Abs. 4.2.2). Liegt das Minimum bei $h=0$, was nur unter Vernachlässigung der kurzreichweitigen repulsiven Kräfte möglich ist $(\epsilon=0)$, ist das System vollständig entnetzend und der Film perlt vom Substrat ab, das System ist instabil. Gibt es ein globales Minimum bei einer 
Filmdicke $0<h<\infty$, wofür $A>0$ sein muss, nennt man das System teilweise benetzend. Ist bei einem Film der Dicke $d>h$ außerdem $\Phi^{\prime \prime}(d)<0$, ist das System ebenfalls instabil und der Film entnetzt so, dass sich Tropfen mit endlichem Kontaktwinkel zum Substrat aber auch eine Gleichgewichtslage der Dicke $h$ bilden [See01a]. Ändert sich das Vorzeichen bei einer höheren Schichtdicke noch einmal von $\Phi^{\prime \prime}<0 \mathrm{zu} \Phi^{\prime \prime}>0$, ist das System metastabil, d. h. dünne Schichten sind instabil und dickere Schichten stabil, wobei stabil in diesem Fall meint, dass Entnetzung nicht durch Fluktuationen sondern nur durch Defekte, bzw. Nukleation initiiert werden kann.

Neben der Ähnlichkeit der Strukturen der thermisch aufgedampften PC Schichten mit Strukturen von entnetzenden Polymerfilmen zeigt das Langzeitverhalten der dünnen Schichten, das weiter unten diskutiert wird, entnetzungsartiges Verhalten, d. h. mit zunehmender Zeit wird immer mehr Substrat freigelegt und das Material zieht sich zusammen. Dickere Schichten zeigen dieses Verhalten nicht, ihre Topographie ändert sich nicht im Verlauf der beobachteten Zeit. Bei thermisch aufgedampftem PC auf Si, $\mathrm{SiO}_{2}$ und GaAs handelt es sich also um metastabile Systeme. Das Verhalten ist dabei nur entnetzungsartig und keine wirkliche Entnetzung, da mit dem Begriff Entnetzung eigentlich das Aufbrechen eines zunächst geschlossenen Films gemeint ist und hier bei den dünnen Schichten keine geschlossenen Schichten beobachtet worden sind. Dennoch wird der Begriff Entnetzung im Folgenden auch für die hier beobachtete Entwicklung verwendet.

Für PC auf PC ist ein solches Verhalten nicht zu beobachten, hier sind alle Schichten unabhängig von ihrer Dicke stabil, was einer negativen Hamaker-Konstante entspricht. Da sich die Struktur vom PC durch das Aufdampfen nicht stark ändert, sind auf PC Substrat die Wechselwirkungen zwischen aufgedampftem Material und Substrat vergleichbar mit den internen van-der-Waals Kräften und es gibt keine treibende Kraft für Entnetzungsprozesse.

Die Strukturbildung bei den thermisch aufgedampften PC Schichten ist demnach zum einen von der Deposition und dem Wachstum mit den damit verbundenen Prozessen und zum anderen von Entnetzungsprozessen beeinflusst. Da die Entnetzungsprozesse aber sehr langsam sind und sich erst auf Zeitskalen von Wochen und Monaten bemerkbar machen (wie man es in der zeitlichen Entwicklung der Struktur in den Abb. 6.18 und 6.19 sieht), während die Schichtherstellung innerhalb einiger Stunden stattfindet, können die Entnetzungsprozesse bei der Betrachtung der anfänglichen Strukturen, die innerhalb von $24 \mathrm{~h}$ nach der Herstellung aufgenommen werden, vernachlässigt werden. Aber natürlich spielen die Wechselwirkungen zwischen Film und Substrat auch bei den Wachstumsprozessen eine wichtige Rolle und entscheiden darüber, ob es zu Lagen- oder Inselwachstum kommt. Bei Systemen, die zu Entnetzung neigen, ist Inselwachstum zu erwarten. Bei der Schichtherstellung mittels physikalischer Gasphasenabscheidung geben die Teilchen bei der Kondensation auf dem Substrat einen Teil ihrer Energie ab und diffundieren anschließend entsprechend ihrer verbleibenden Energie auf der Oberfläche, um einen energetisch möglichst günstigen Platz (beim Inselwachstum eine Insel) zu erreichen. Später stattfindende Diffusionsprozesse wie Volumendiffusion und Oberflächendiffusion hängen dagegen ebenso wie Fließprozesse von der Substrattemperatur oder der Umgebungstemperatur ab und sind bei Raumtemperatur dementsprechend langsam. Treffen während des Wachstums Inseln aufeinander, setzt Koaleszenz ein, wodurch der Film stabilisiert werden kann, wie es auch bei metallischen Gläsern [May98], 
laserdeponierten Polymeren [Hac04] und in Computersimulationen [Vre06] beobachtet worden ist. In den Bereichen, wo die Koaleszenz noch nicht vollständig abgeschlossen ist, können im Laufe der Zeit Entnetzungsprozesse oder diffusive und auf Diffusion basierende Prozesse wie Ostwald-Reifung beginnen, deren Einsetzen man z. B. durch Erhöhung der Substrattemperatur und die damit verbundene Erhöhung der Dynamik früher beginnen und schneller ablaufen lassen kann. Da in diesem Stadium dieselben Wechselwirkungen die Struktur beeinflussen, wie in den Anfangsstadien der Entnetzung eines vorher homogenen Films, sind sich die entstehenden Morphologien sehr ähnlich.

Diese Überlegungen sind auch in die Entwicklung der Kontinuumsmodellierung in Abs. 4.2.1 eingegangen. Neben der Deposition beinhaltet das Modell nur Oberflächendiffusion als Wachstumsprozess, die durch Gradienten im chemischen Potential getrieben wird. Zum chemischen Potential trägt neben der Krümmung der Oberfläche nur das effektive Grenzflächenpotential bei. Die Simulationen eines instabilen Systems $(A>0)$ liefern (nach geeigneter Reskalierung der Topographien, vgl. Abs. 4.2.3) sowohl qualitative als auch quantitative Übereinstimmung mit thermisch aufgedampften PC Schichten auf GaAs. Die Topographien sind sich sehr ähnlich und zeichnen sich durch grabenartige Vertiefungen bei den dünneren Schichten aus, die mit steigender Schichtdicke immer rundere Formen annehmen und an Tiefe verlieren. Auch die Rauigkeitsentwicklungen von Simulation und Experiment stimmen gut überein, wie es in Abb. 7.2 dargestellt ist. Das vorgestellte Kontinuumsmodell (Gl. (4.8)) ist also in der Lage, das Wachstum der thermisch aufgedampften PC Schichten (zumindest auf GaAs) über mindestens zwei Größenordnungen der Schichtdicke zu beschreiben. Weitere Tests haben gezeigt, dass Modelle mit viskosem Fließen, das bei früheren Entnetzungsexperimenten dominierend ist [Bec03], und Deposition nicht in der Lage sind, die experimentell beobachteten Strukturen zu reproduzieren. Wesentlich für die Strukturbildung ist demnach tatsächlich das effektive Grenzflächenpotential und insbesondere die Hamaker-Konstante. Ohne den Beitrag des Grenzflächenpotentials $\Phi$ vereinfacht sich die Wachstumsgleichung (4.8) zur linearen MBE Gleichung, die bereits von Wolf und Villain diskutiert worden ist [Wol90]. Diese zeigt ein Anwachsen der Rauigkeit mit der Schichtdicke gemäß einem Potenzgesetz und weist vor allem kein Maximum in der Rauigkeitsentwicklung auf, wie es hier in den Experimenten beobachtet wird. Ein solches Potenzgesetz ist typisch für kinetische Aufrauung [Bar95], die in vielen Systemen, sowohl bei Metallen als auch bei Polymeren, beobachtet wird [Col94; Zha00; Ben00; Vre06]. Ein solches Verhalten zeigt hier die Entwicklung eines stabilen Systems $(A<0)$, bei dem die Rauigkeit über den gesamten untersuchten Dickenbereich dem Potenzgesetz $\sigma \propto d^{0,25}$ folgt (vgl. Abb. 7.5). Hier ist auch in den anderen Größen, wie der Korrelationslänge $R_{c}$, dem Rauigkeitsexponenten $\alpha$ und dem Abfall des Fourierspektrums wie bei den thermisch aufgedampften PC Schichten auf PC keine Dickenabhängigkeit zu erkennen, wohingegen im instabilen System und in den Experimenten auf $\mathrm{Si}, \mathrm{SiO}_{2}$ und $\mathrm{GaAs}$ deutliche Unterschiede zwischen den dünnen und den dickeren Schichten sichtbar sind.

Die instabilen Systeme in den Experimenten und in der Simulation haben alle ihren maximalen Rauigkeitswert bei Schichtdicken unter $30 \mathrm{~nm}$ und mit steigender Schichtdicke fällt die Rauigkeit schnell ab (vgl. Abs. 6.2.2). Der Anstieg der Rauigkeit bis zum Maximum ist in den Experimenten schwer zu beobachten, da in diesem Dickenbereich nur wenige Proben hergestellt worden sind. In der Simulation dagegen ist die Datendichte sehr viel höher (Aufnahme der Topographie etwa alle $0,7 \mathrm{~nm}$ ) und die 

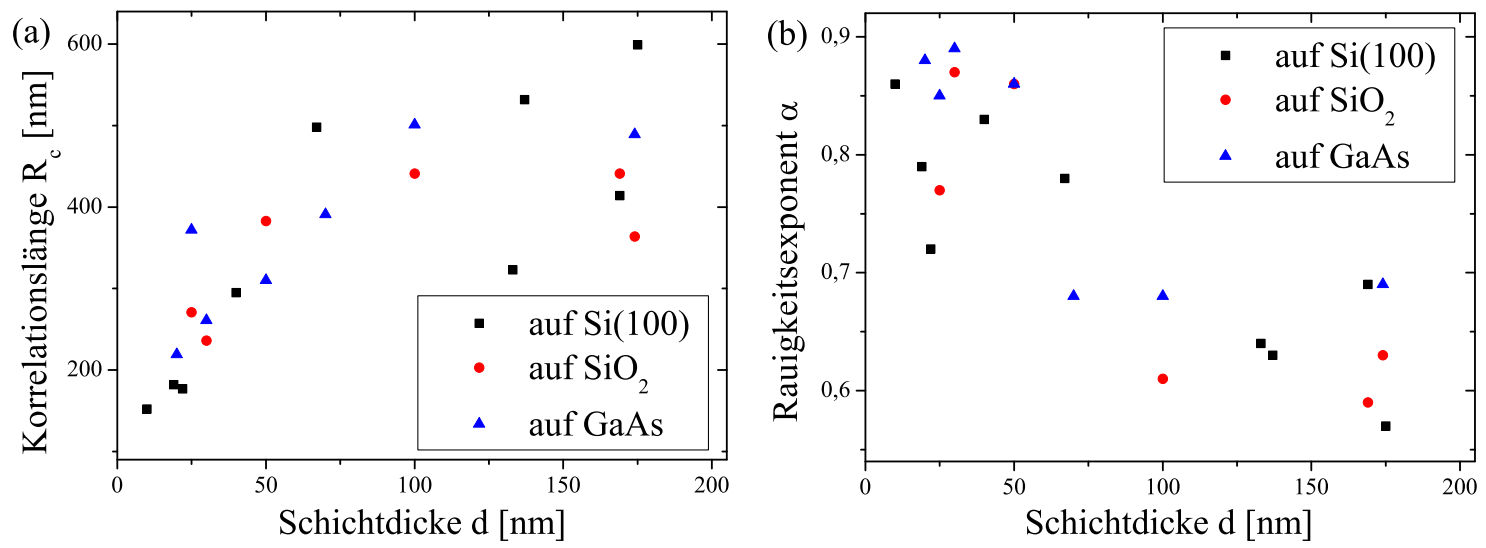

Abbildung 9.4: (a) Abhängigkeit der Korrelationslänge $R_{c}$ von der Schichtdicke für thermisch aufgedampftes $\mathrm{PC}$ auf $\mathrm{Si}, \mathrm{SiO}_{2}$ und GaAs. Mit zunehmender Schichtdicke steigt $R_{c}$ an. (b) Rauigkeitsexponent $\alpha$ von thermisch aufgedampftem $\mathrm{PC}$ in Abhängigkeit von der Schichtdicke. Bei dünnen Schichten gilt $\alpha \approx 0,85$, während bei dicken Schichten $\alpha \approx 0,65$ ist.

Strukturentwicklung während des Rauigkeitsanstiegs ist genau zu beobachten. Auf dem anfänglich leeren Substrat $(\sigma=0 \mathrm{~nm})$ bilden sich viele getrennte Inseln, die mit zunehmender Teilchenzahl anwachsen, wodurch die Rauigkeit schnell ansteigt. Inseln, die nahe aneinander liegen, bilden längliche Strukturen, sobald sie sich berühren. So entsteht eine Art Netzwerk, bei dem die Zwischenräume zunächst eher länglich sind. Die maximale Rauigkeit ist etwa dann erreicht, wenn das Netzwerk geschlossen ist und die Strukturen mehr in die Breite wachsen. Durch das Wachstum des Netzwerks werden die Zwischenräume immer kleiner und nehmen rundere Form an. Außerdem werden sie mit Material aufgefüllt, wodurch die Rauigkeit schnell abfällt. Außerdem werden die typischen Abstände zwischen den Strukturen, die aus der Korrelationslänge $R_{c}$ bestimmt werden können, größer, da diese anfangs den Abstand der getrennten Inseln angeben, aber mit wachsender Schichtdicke immer größere Bereiche zusammenhängen, zwischen denen dann der typische Abstand gemessen wird. Der Anstieg von $R_{c}$ ist in Abb. 9.4(a) dargestellt. Bis zu einer Schichtdicke von etwa $100 \mathrm{~nm}$ steigt der typische Abstand von ca. $180 \mathrm{~nm}$ auf $500 \mathrm{~nm}$ an und bleibt dann bei diesem Wert. Bei den dickeren Schichten ist eine Bestimmung von $R_{c}$ allerdings auch schwierig, da das erste Maximum der Höhen-Höhen-Korrelationsfunktionen nicht mehr deutlich zu erkennen ist (vgl. Abb. 6.8, 6.11, 6.14). In Abb. 9.4(b) ist der Rauigkeitsexponent $\alpha$ für verschiedene Schichtdicken aufgetragen. Für dünne Schichten ist $\alpha \approx 0,85$, während bei dicken Schichten $\alpha \approx 0,65$ gilt. Auch in den Simulationen ändert sich $\alpha$ mit der Schichtdicke, allerdings von $\alpha \approx 0,8$ auf $\alpha \approx 0,95$. Ebenso ändert sich der Abfall des Fourierspektrums, der für dicke Schichten etwa einem $q^{-4}$ Verlauf folgt, während er für dünne Schichten steiler abfällt. Einen $q^{-4}$ Verlauf des Fourierspektrums findet man für Wachstumsprozesse, die von Oberflächendiffusion dominiert sind [Her50; Mul57; Bar95]. Steilere Abfälle deuten darauf hin, dass nicht nur lineare Prozesse ${ }^{3}$ beteiligt sind. Für

\footnotetext{
${ }^{3}$ Ein linearer Prozess ist hier ein Prozess, der im Kontinuumsmodell durch einen in $h$ oder in höheren Ableitungen von $h$ linearen Term modelliert werden kann.
} 
dicke Schichten ist der Einfluss des effektiven Grenzflächenpotentials klein und kann vernachlässigt werden, damit bleibt im Kontinuumsmodell neben Deposition nur noch der Term für krümmungsinduzierte Oberflächendiffusion. Dafür erwartet man einen $q^{-4}$ Verlauf des Fourierspektrums und einen Rauigkeitsexponenten $\alpha=1$ [Bar95], wie es hier auch beobachtet wird. In den Experimenten findet man dagegen $\alpha \approx 0,65$, was durch zusätzliche Beiträge von Adatom-konzentrationsinduzierter Oberflächendiffusion erklärt werden kann [Bar95]. Ein solcher Wert wurde im Übrigen auch bei laserdeponierten PC Schichten gefunden [Hac04; Vre05]. Wie weiter oben bereits erwähnt, erwartet man für solche Systeme kinetische Aufrauung, also ein Anwachsen der Rauigkeit mit der Schichtdicke, welches hier weder in der Simulation noch im Experiment beobachtet wird. Es ist nicht auszuschließen, dass zu höheren Schichtdicken hin kinetische Aufrauung einsetzt. Der experimentelle Aufbau erlaubt aber im Moment keine Präparation dickerer Schichten. Hier sind weitere Untersuchungen und ein Umbau der Apparatur notwendig.

\subsubsection{Zeitliche Entwicklung der Topographie}

Die zeitliche Entwicklung der Strukturen von dünnem thermisch aufgedampften PC, die exemplarisch anhand einer $20 \mathrm{~nm}$ dicken PC Schicht auf Si in Abb. 6.18 dargestellt ist, zeigt, dass diese auf $\mathrm{Si}, \mathrm{SiO}_{2}$ und GaAs Substraten nicht stabil sind, sondern über einen Zeitraum von mehreren Tagen, bzw. Wochen und Monaten immer mehr Substratfläche freilegen (vgl. Abb. 6.19). Der Anteil der unbedeckten Fläche steigt dabei innerhalb von $690 \mathrm{~h}$ von anfangs etwa $10 \%$ bis auf $50 \%$ an. Es handelt sich also um (langsame) Entnetzungsprozesse (wobei hier diffusive Prozesse nicht ausgeschlossen werden können, diese sind thermisch aktiviert und bei Raumtemperatur daher sehr langsam). Da dickere Schichten mit $d \gtrsim 40 \mathrm{~nm}$ auf diesen Substraten stabil sind und auch nach Monaten keine messbare Veränderung aufweisen, handelt es sich um metastabile Systeme im Sinne der weiter oben genannten Definitionen. Auf PC Substraten ist bei keiner Schichtdicke eine Veränderung der Topographie mit der Zeit zu erkennen, es handelt sich demnach um ein stabiles System.

Die Dynamik der Entnetzung kann durch Temperaturbehandlung beschleunigt werden, so ist z. B. eine $18 \mathrm{~nm}$ dicke PC Schicht auf Si nach Lagerung im Ofen bei $70{ }^{\circ} \mathrm{C}$ nach 7 Tagen vollständig entnetzt, d. h. der Film besteht aus einzelnen, isolierten Inseln. Aber auch bei Raumtemperatur findet Entnetzung statt. Wie bereits weiter oben erwähnt, ist Entnetzung ein bei Polymeren nahe ihrer Glasübergangstemperatur $T_{g}$ und bei Flüssigkeiten bekanntes Phänomen [Rei01]. Die Glasübergangstemperatur von unbehandeltem $\mathrm{PC}$ beträgt $T_{g} \approx 150{ }^{\circ} \mathrm{C}$, d. h. es liegt weit über der Lagerungstemperatur. Durch das thermische Aufdampfen wird demnach die Glasübergangstemperatur deutlich reduziert, da sonst keine Entnetzung stattfinden könnte. Dafür kommen im Wesentlichen zwei Effekte in Frage, zum einen die verkürzte Kettenlänge und zum anderen die geringe Schichtdicke. Von Polymeren ist bekannt, dass sich die Glasübergangstemperatur sowohl mit abnehmender Molmasse [Fox55; Spe85] als auch mit der Schichtdicke [Her01; For01] verringert, wobei die Abhängigkeit von der Schichtdicke immer noch kontrovers diskutiert wird, da sie vom jeweiligen System und der SubstratFilm-Wechselwirkung abhängt [Tor00; For01]. Für Systeme, die eine Abnahme von $T_{g}$ mit der Schichtdicke zeigen, wie das oft untersuchte System Polystyrol auf Si Substra- 
ten mit unterschiedlich dicker Oxidschicht, kann der Einfluss der Schichtdicke durch die empirische Funktion

$$
T_{g}=T_{g}^{0}\left(1-\frac{d_{0}}{d}\right)
$$

beschrieben werden, wobei $T_{g}^{0}$ die Glasübergangstemperatur einer Volumenprobe angibt und $d_{0}$ eine Konstante bezeichnet, die je nach System im Bereich 0,5 nm bis $5 \mathrm{~nm}$ liegt [Her01]. Für PS auf $\mathrm{SiO}_{2}$ wird z. B. eine Abnahme von $T_{g}$ um etwa $20 \%$ beobachtet, die mit $d_{0}=0,8 \mathrm{~nm}$ beschrieben werden kann [Her01]. In Abb. 9.5(a) ist die Abnahme von $T_{g}$ gemäß dieser Gleichung mit den Parametern $d_{0}=1 \mathrm{~nm}$ und $T_{g}^{0}=50{ }^{\circ} \mathrm{C}$ aufgetragen. Unterhalb von $50 \mathrm{~nm}$ Schichtdicke wird die Abnahme von $T_{g}$ mit der Schichtdicke stärker.

Um den Einfluss des Molekulargewichts $M$ auf die Glasübergangstemperatur bestimmen zu können, haben Fox und Loshaek aus dem Zusammenhang von Volumen, Temperatur und Molekulargewicht einerseits und von Volumen und Temperatur bei $T_{g}$ andererseits die folgende Abhängigkeit von $T_{g}$ von $M$ bestimmt [Fox55]:

$$
\frac{1}{T_{g}}=\frac{1}{T_{g, \infty}}+\frac{K}{T_{g, \infty}^{2} M}
$$

Dabei bezeichnen $T_{g, \infty}$ die Glasübergangstemperatur von quasi unendlich langen Ketten und $K$ eine Ratenkonstante. In der Literatur findet man für diese Größen Werte zwischen $150{ }^{\circ} \mathrm{C} \leq T_{g, \infty} \leq 170{ }^{\circ} \mathrm{C}$ und $1 \times 10^{5} \mathrm{gK} / \mathrm{mol} \leq K \leq 6 \times 10^{5} \mathrm{gK} / \mathrm{mol}$ [Rob02b; Ada76]. In Abb. 9.5(b) sind die Grenzfälle dieser Literaturdaten und die Werte von Adam et al. [Ada76] aufgetragen, aus denen man für die thermisch aufgedampften PC Schichten mit einem Molekulargewicht von 1000 - 2000 g/mol eine Glasübergangstemperatur von $T_{g} \approx 50{ }^{\circ} \mathrm{C}$ abschätzen kann. Diese ist auch als Ausgangspunkt für den Einfluss der Schichtdicke verwendet worden.

Da sowohl die Molmasse als auch die Schichtdicke die Glasübergangstemperatur beeinflussen können, müssen beide Effekte kombiniert werden. So kann für die dünnen Filme eine Absenkung von $T_{g}$ bis auf Raumtemperatur erklärt werden. Die Zunahme von $T_{g}$ mit der Schichtdicke kann ein weiterer Grund dafür sein, dass die dickeren Schichten stabil bleiben.

\subsection{Lokale mechanische Eigenschaften}

Neben der Untersuchung der Topographie bietet ein AFM die Möglichkeit, elastische Eigenschaften zu untersuchen. Dafür sind immer neue Techniken und Betriebsmodi wie Kraft-Abstandskurven und damit zusammenhängend „Force Volume Imaging“ [Rad94], der „Force Modulation Mode“ [Mai91], die akustische Kraftmikroskopie [Rab94a] oder HarmoniX ${ }^{T M}$ [Sah07] entwickelt worden. Mit Hilfe von Kraft-Abstandskurven sind z. B. Zellen [AH98], die Glasübergangstemperatur von Polymeren [Bli02], nanomechanische Eigenschaften von Polymeren [Cap08], Gewebe von Gelenkknorpeln [Sto04], Metalle [Kra00; Hue94] und Polymermischungen [Rag00] untersucht worden. Bei der Untersuchung der thermisch aufgedampften PC Schichten mit Kraft-Abstandskurven hat sich gezeigt, dass diese so weich sind, dass selbst bei kleinen Auslenkungen des Cantilevers 

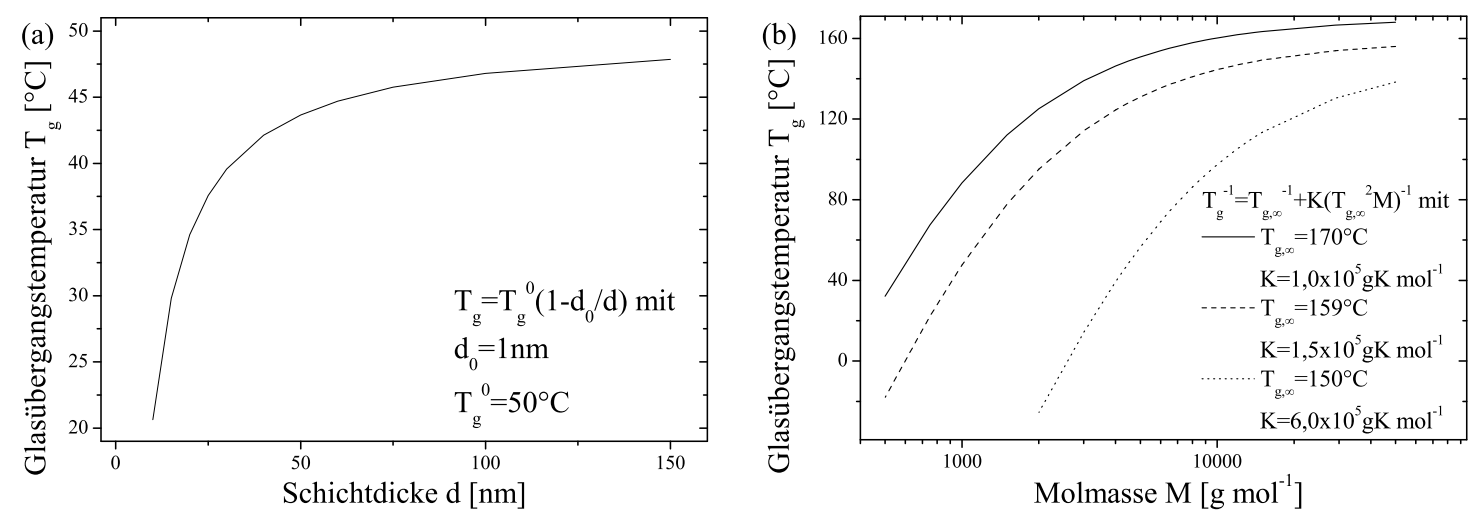

Abbildung 9.5: (a) Abnahme der Glasübergangstemperatur $T_{g}$ mit der Schichtdicke nach der empirischen Formel $T_{g}=T_{g}^{0}\left(1-\frac{d_{0}}{d}\right)$ nach [Her01] mit $d_{0}=1 \mathrm{~nm}$ und $T_{g}^{0}=50^{\circ} \mathrm{C}$. (b) Abnahme von $T_{g}$ mit der Molmasse (logarithmisch aufgetragen) nach der Vorhersage von Fox und Loshaek [Fox55]: $\frac{1}{T_{g}}=\frac{1}{T_{g, \infty}}+\frac{K}{T_{g, \infty}^{2} M}$ mit verschiedenen Abschätzungen nach Daten aus der Literatur (Grenzwerte der Literaturdaten und Daten von Adam et al.) [Rob02b; Ada76].

plastische Verformung auftritt. Ziel war es, mechanische Heterogenitäten auf kleinen Längenskalen, die in Computersimulationen an Polymerfilmen [Yos04] und an metallischen Gläsern [May09] beobachtet worden sind, experimentell zu untersuchen. Die mit Hilfe der Kraft-Abstandskurven erstellten Karten der elastischen Eigenschaften zeigen auf PC große Variationen, die aber auch durch unterschiedliche plastische Verformung erklärt werden können und nicht nur durch heterogene Eigenschaften. Die Verteilung der Werte ist dabei jeweils gaußverteilt, wie man es für statistische Schwankungen um einen Wert erwarten kann. Außerdem können sich die Messpunkte durch die Verformung gegenseitig beeinflussen, wenn Material an einem Messpunkt bis zum nächsten Messpunkt verschoben wird. Bei den $70 \mathrm{~nm}$ dicken thermisch aufgedampften PC Schichten sind praktisch keine Unterschiede durch die verschiedenen Substrate zu erkennen, weder in den Steigungen der Kraft-Abstandskurven, noch in der Größe der Variationen oder in der Stärke der plastischen Verformung. Diese Schichtdicke reicht also aus, um den Einfluss der Grenzfläche zum Substrat klein werden zu lassen und nur die Eigenschaften der PC Schicht zu untersuchen. Im Vergleich zu den PC Schichten sind die PC Substrate etwas härter, wie es aufgrund der längeren Kettenlänge zu erwarten ist, allerdings sind auch die Variationen größer, was auf eine größere Inhomogenität schließen lässt. Auch auf $\mathrm{SiO}_{2}$ sind Variationen erkennbar, ohne dass die Oberfläche durch die Messung verändert wird. Dies zeigt, dass die Methode im Prinzip funktioniert, mit den verwendeten Cantilevern auf den PC Schichten aber nicht gut anwendbar ist. Eine mögliche Lösung wären Cantilever mit noch geringerer Federkonstante, mit denen man aber meist nur im Kontaktmodus die Topographie messen kann und solche Messungen führen auf den weichen PC Schichten wiederum zur Deformation. D. h. man kann nicht mit dem gleichen Cantilever die Topographie nach der Messung aufnehmen, um zu kontrollieren, ob sich die Oberfläche verändert hat.

Ein sehr ähnliches Problem ergibt sich bei den AFAM Messungen. Die Tests auf den 
Kalibrierungsproben und vor allem Messungen an metallischen Gläsern von H. Wagner zeigen, dass die neue Erweiterung betriebsbereit, aber auf den PC Schichten nicht gut anwendbar ist. Außerdem geben die Messungen an amorphen $\mathrm{SiO}_{2}$ im Vergleich zu kristallinen Strontiumtitanat und Saphir Hinweise darauf, dass die lokalen Variationen der elastischen Eigenschaften auf amorphen Oberflächen größer als auf kristallinen Oberflächen sind. Für die PC Schichten lassen sich hier noch keine Aussagen machen. AFAM ist eine Technik, die sich gut für härtere Proben wie z. B. die metallischen Gläser eignet, wohingegen für die weichen Polymere die neue HarmoniX ${ }^{T M}$ Technik von Veeco Instruments $\mathrm{GmbH}$ vielversprechender scheint, die für genau solche Untersuchungen entwickelt worden ist. Mit HarmoniX ${ }^{T M}$ sollen nach Angabe von Veeco Instruments Proben mit einem E-Modul im Bereich von $10 \mathrm{MPa}$ bis $10 \mathrm{GPa}$ untersucht werden können, wodurch sie wiederum nicht für härtere Proben wie die metallischen Gläser anwendbar ist.

\subsection{Diskussion der MD Simulationsergebnisse}

Im Folgenden sollen die Ergebnisse der MD Simulationen zur Dynamik von dünnen, amorphen, freistehenden Polymerfilmen diskutiert und interpretiert werden. Bevor die eigentliche Dynamik betrachtet wird, soll geklärt werden, inwieweit die Ergebnisse von der vorhergehenden Behandlung, also von Herstellung und Relaxation, abhängen.

Die Ergebnisse der Strukturuntersuchungen, die exemplarisch in Abs. 8.1 vorgestellt sind, zeigen, dass alle präparierten Zellen amorph sind. Die Paarkorrelationsfunktionen zeigen den für amorphe Festkörper und Flüssigkeiten typischen Verlauf [Ell90]. Mit zunehmender Temperatur werden die Maxima aufgrund der höheren thermischen Fluktuationen etwas breiter und niedriger. Die Paarkorrelationsfunktionen werden während eines Simulationslaufes regelmäßig ausgewertet und es sind bei keiner Temperatur Anzeichen von Kristallisation aufgetreten. Somit sind die Filme dünn, freistehend und amorph und bleiben dies auch während der gesamten Simulationen.

Die Glasübergangstemperatur $T_{g}$ liegt für die zur Dynamikuntersuchung hergestellten Proben bei $T_{g} \approx 0,44 \epsilon / \mathrm{k}_{\mathrm{B}}$. Dabei ist $T_{g}$ abhängig von der Kühl-, bzw. Heizrate und steigt kontinuierlich mit zunehmender Abkühlrate an, d. h. je schneller eine Probe abgeschreckt wird, desto höher liegt die Glasübergangstemperatur, wie man es für Gläser erwartet [El190]. Für die höchste untersuchte Abkühlrate findet man die höchste Glasübergangstemperatur $T_{g}\left(\frac{9 \epsilon / \mathrm{k}_{\mathrm{B}}}{10^{6} \Delta t}\right) \approx 0,46 \epsilon / \mathrm{k}_{\mathrm{B}}$ und bei der niedrigsten Abkühlrate $T_{g}\left(\frac{9 \epsilon / \mathrm{k}_{\mathrm{B}}}{10^{8} \Delta t}\right) \approx 0,42 \epsilon / \mathrm{k}_{\mathrm{B}}$.

Für beide Herstellungsvarianten (vgl. Abs. 5.2.2) zeigt die zeitliche Entwicklung der Gesamtenergie während der eigentlichen Simulationen einen, innerhalb der Schwankungen, konstanten Verlauf. Dies ist ein deutlicher Hinweis darauf, dass die Relaxationszeiten vor Beginn der eigentlichen Simulationen lang genug sind. Dies bestätigt auch ein Testlauf mit verdoppelter Relaxationszeit, dessen Ergebnisse innerhalb des Fehlers nicht von denen der kürzeren Relaxation abweichen. Es gibt also keine Hinweise darauf, dass die Ergebnisse auf einen zu kurz relaxierten Ausgangspunkt zurückzuführen sind. Auch Größeneffekte durch zu kleine Simulationszellen können aufgrund von Testläufen mit verdoppelter Zellengröße, die zu den gleichen Ergebnissen führen, ausgeschlossen wer- 
den. Die Zelle ist außerdem so groß gewählt, dass die Enden einer Kette im vollständig gestreckten Zustand nicht miteinander wechselwirken.

\subsubsection{Betrachtung einer einzelnen Kette auf der Oberfläche}

Die Betrachtung einer einzelnen Kette auf der Oberfläche bietet die Möglichkeit, die Bewegung im Detail zu beobachten und qualitativ zu beschreiben. Sie ist daher besonders zur Visualisierung der Kettenbewegung geeignet. Die Fluktuationen, die alle thermisch aktivierten Prozesse, also auch die Diffusion hier, beeinflussen, sorgen vor allem bei der tieferen Temperatur von $T=0,40 \epsilon / \mathrm{k}_{\mathrm{B}}$ dafür, dass für die einzelne Kette keine effektive Diffusionskonstante bestimmt werden kann, da die Schwankungen die mittlere Verschiebungsweite dominieren (vgl. Abb. 8.5(a) und (b)). Es fällt auf, dass es Zeiträume mit großen Änderungen gibt, aber auch Zeiträume, in denen sich die Kette fast gar nicht bewegt. Das äußert sich auch im End-zu-End Abstand und im Gyrationsradius (Abb. 8.4), in denen ebenfalls unterschiedlich starke Änderungen zu verschiedenen Zeiten zu beobachten sind. Wie man in den zugehörigen Momentaufnahmen erkennen kann, bewegt sich die Kette ähnlich wie eine Raupe auf der Oberfläche. Dieser raupenartige Bewegungsmechanismus ist so nur auf der Oberfläche möglich, da im Volumen die Bewegungsfreiheit durch die umliegenden Ketten eingeschränkt ist. In den Stadien größerer Bewegung streckt sich die Kette etwas aus, verändert also ihre Konformation in einen länglichen Zustand, wodurch End-zu-End Abstand und Gyrationsradius erhöht sind und zieht sich dann wieder zusammen, da der gestreckte Zustand aufgrund der niedrigeren (Konfigurations-) Entropie ungünstiger ist. Daher zieht sich die Kette zu Beginn der Simulation auch zusammen. Die meiste Zeit liegt die Kette flach auf der Oberfläche, wie es auch in Experimenten [Suk00] und anderen Simulationen beobachtet wird [Qia07; Yan06; Des07; Muk08], in denen bei höheren Temperaturen die Abhängigkeit des Diffusionskoeffizienten von der Kettenlänge untersucht worden ist.

Bei $T=0,44 \epsilon / \mathrm{k}_{\mathrm{B}}$ ist neben den Fluktuationen eine effektive Bewegung der Einzelkette zu erkennen, der Bewegungsmechanismus ist dabei unverändert. Die Bewegungsweiten sind sowohl für die einzelne Kette als auch für die Ketten in der Zelle aufgrund der höheren Temperatur deutlich größer als bei $T=0,40 \epsilon / \mathrm{k}_{\mathrm{B}}$. Der Temperatureinfluss und der Unterschied zwischen Oberfläche und Volumen werden im folgenden Abschnitt ausführlich diskutiert.

\subsubsection{Einfluss der Oberflächen auf die diffusive Dynamik}

Der Einfluss der Oberflächen wird bei Betrachtung der Tiefenprofile der effektiven Diffusionskonstante in Abb. 8.8 deutlich, welche ein Maßs für die Mobilität der Ketten ist. Die erhöhte Mobilität im Oberflächenbereich ist dabei in guter Übereinstimmung mit Experimenten [Zap01; Fak08] und Simulationen [Pet06; Pet07] von Polymeren. In Experimenten ist die Dynamik von Polymeroberflächen z. B. durch das Einsinkverhalten von metallischen Nanopartikeln in einen Polymerfilm [Zap01] oder durch die Ausheilung von gezielt durch Nanopartikel an der Oberfläche eingebrachte Deformationen [Fak08] untersucht worden. Beide Methoden weisen auf eine erhöhte Mobilität an der Oberfläche hin. Jedoch gibt es in Experimenten mit dielektrischer Spektroskopie an dünnen Polymerfilmen $(d>10 \mathrm{~nm})$ keine Hinweise auf eine erhöhte Mobilität, zumindest gibt 
es keine Verschiebung der mittleren Relaxationszeit und keine Verbreiterung des dynamischen Glasübergangs [Ser08]. In diesen Experimenten wird allerdings der Einfluss der Schichtdicke auf die Gesamtdynamik im Film, wobei die dielektrische Spektroskopie im Wesentlichen Rotationsdynamik testet, untersucht und es können keine Aussagen über Oberflächeneffekte gemacht werden. Dafür können Substrateinflüsse, die je nach Wechselwirkung auch den gegenteiligen Effekt einer Reduktion der Mobilität zeigen können, stärkere Auswirkungen haben. In Simulationen zum Einfluss der Schichtdicke auf die Glasübergangstemperatur von dünnen Polymerfilmen ist die Mobilität in der Nähe einer freien Oberfläche ebenfalls erhöht [Tor00; Pet06; Pet07]. Die Tiefenprofile der effektiven Diffusionskonstante haben außerdem eine große Ähnlichkeit zu Simulationen von metallischen Gläsern [Ba196; Böd99; Vau05], die auch einen deutlichen Anstieg an den Oberflächen im Vergleich zum Volumen zeigen. Das deutet auf eine gewisse Universalität des Phänomens in amorphen Systemen hin.

Die Erhöhung der Mobilität an den Oberflächen, also das Verhältnis von Oberflächendiffusion zu Volumendiffusion, wird mit zunehmender Temperatur kleiner und scheint oberhalb von $T \approx 0,5 \epsilon / \mathrm{k}_{\mathrm{B}}$ einen Grenzwert zu erreichen. Dabei muss beachtet werden, dass die Ketten bei hohen Temperaturen stärker vermischt werden, also Ketten aus dem Volumen an die Oberfläche diffundieren und umgekehrt. Da für die Mittelung zur Bestimmung der Tiefenprofile die z-Koordinaten der Schwerpunkte der Ketten zu Beginn der Simulation verwendet werden, nähern sich Volumen- und Oberflächenwerte einander an. Die Verwendung der anfänglichen z-Koordinaten ist in Simulationen so etwas wie Tracer-Diffusion in Experimenten, in denen man bestimmte Objekte zu Beginn markiert und dann ihre Bewegung verfolgt. Eine andere Möglichkeit, die Oberflächendiffusion in Simulationen zu untersuchen, wäre nur solche Ketten zu beachten, die während der gesamten Simulationszeit an der Oberfläche verweilen. Diese Methode würde aber eine im Vergleich zu Experimenten zu hohe Diffusionskonstante ergeben und in der vorliegenden Arbeit geht es gerade auch darum, solche Größen zu betrachten, die experimentell zugänglich sind. Daher wird die Diffusion auch nicht wie in vielen anderen Simulationen in einen parallelen und einen senkrechten Anteil zur Oberfläche aufgeteilt [Var02; Pet07; Des07], da experimentell auch alle Richtungen betrachtet werden.

An dieser Stelle soll noch einmal darauf hingewiesen werden, dass sich die Dichte im Oberflächenbereich nicht von der Dichte im Volumen unterscheidet, weshalb die Mobilitätserhöhung nicht durch Konzepte des freien Volumens [Tur58] erklärt werden kann, dennoch ist die Behinderung durch die Umgebung an der Oberfläche geringer, da über den Oberflächen keine Teilchen vorhanden sind. Des Weiteren gibt es trotz der deutlichen Unterschiede zwischen Volumen- und Oberflächeneigenschaften einen kontinuierlichen Übergang und keinerlei Hinweise auf verschiedene Phasen, vor allem keine Phasengrenze. Da der Glasübergang ein kontinuierlicher Übergang ist, ist eine Phasengrenze aber auch nicht zu erwarten [El190]. Ob es sich beim Glasübergang um einen (thermodynamischen) Phasenübergang handelt, ist in der Literatur noch nicht endgültig geklärt [Hec08].

Neben dieser qualitativen Beschreibung erlaubt die Temperaturentwicklung der effektiven Diffusionskonstanten eine quantitative Auswertung in einer Arrhenius-Auftragung, wie sie bereits in Abb. 8.9 dargestellt ist. Aus den Steigungen in einer ArrheniusAuftragung lassen sich die Aktivierungsenthalpien $\Delta H_{D}$ der betrachteten Prozesse bestimmen. Dies ist in Abb. 9.6 verdeutlicht. $\mathrm{Zu}$ tieferen Temperaturen hin ist sowohl 


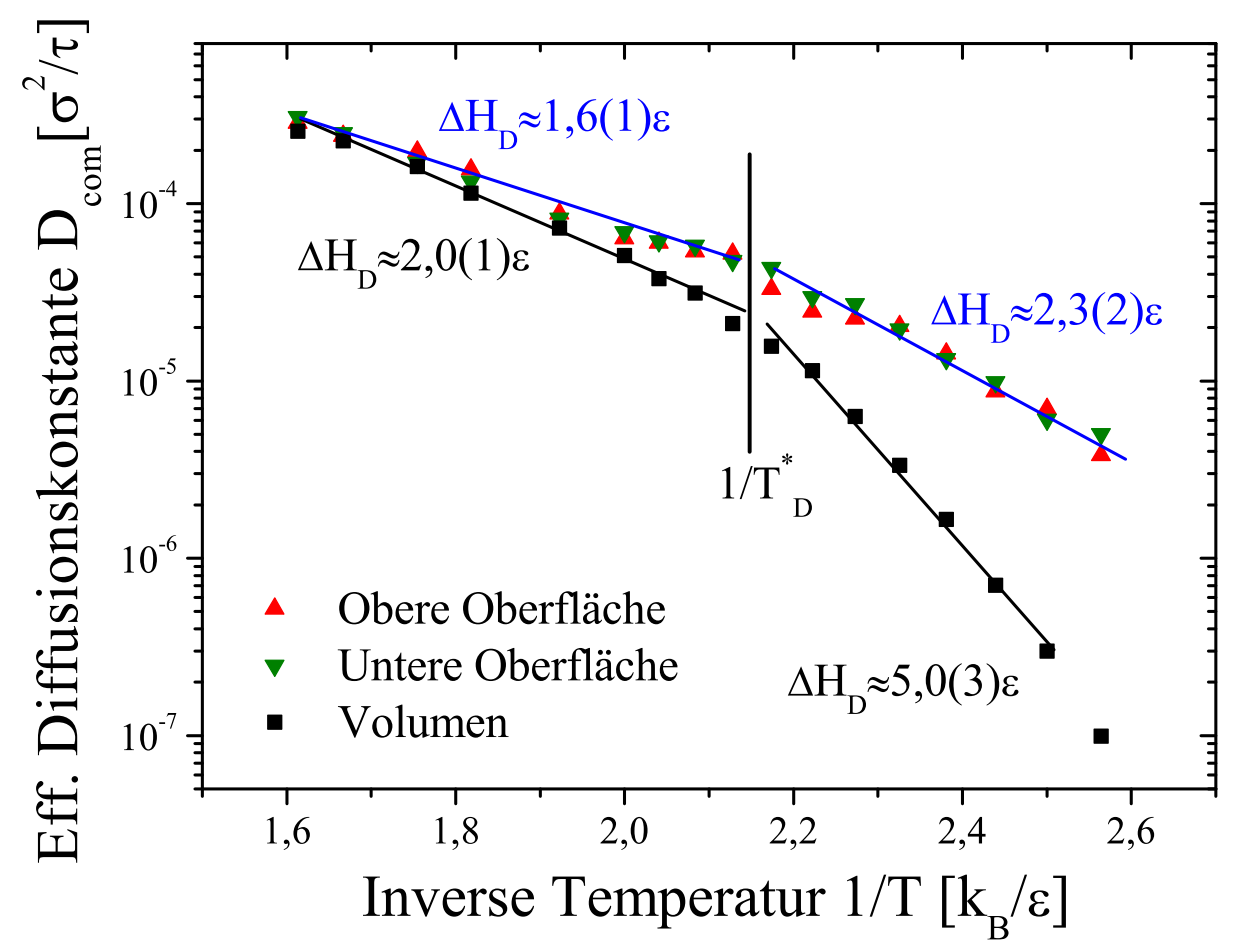

Abbildung 9.6: Arrhenius-Auftragung der effektiven Diffusionskonstante im Volumen (schwarze Quadrate) und in den Oberflächenbereichen (rote (oben) und grüne (unten) Dreiecke) wie in Abb. 8.9 mit zusätzlichen Hilfslinien zur Verdeutlichung der unterschiedlichen Steigungen. Die aus den Steigungen bei hohen und bei niedrigen Temperaturen bestimmten Aktivierungsenthalpien für die unterschiedlichen Bereiche sind mit aufgeführt. Der flüssigkeitsähnliche Bereich bei hohen Temperaturen ist im Volumen und an den Oberflächen klar vom glasartigen Zustand durch veränderte Aktivierungsenthalpien getrennt. Der Übergang erfolgt bei der Temperatur $T_{D}^{*} \approx 0,47 \epsilon / \mathrm{k}_{\mathrm{B}}$.

im Volumen als auch an den Oberflächen eine stärkere Abnahme der effektiven Diffusionskonstante mit der Temperatur zu erkennen. Es sind zwei Temperaturbereiche zu unterscheiden, die durch einen Übergang bei der Temperatur $T_{D}^{*} \approx 0,47 \epsilon / \mathrm{k}_{\mathrm{B}}$ getrennt werden können. Dies äußert sich in unterschiedlichen Steigungen und somit unterschiedlichen Aktivierungsenthalpien im Hoch- und im Niedrigtemperaturbereich. Bei niedrigen Temperaturen findet man $\Delta H_{D} \approx 5,0(3) \epsilon$ im Volumen und $\Delta H_{D} \approx 2,3(2) \epsilon \mathrm{im}$ Oberflächenbereich, wohingegen die Werte bei hohen Temperaturen mit $\Delta H_{D} \approx 2,0(1) \epsilon$ im Volumen und $\Delta H_{D} \approx 1,6(1) \epsilon$ im Oberflächenbereich kleiner sind. Die Übergangstemperatur $T_{D}^{*}$ liegt höher als die Glasübergangstemperatur $\left(T_{g} \approx 0,44 \epsilon / \mathrm{k}_{\mathrm{B}}\right)$ und weist große Ähnlichkeit mit der kritischen Temperatur $T_{c}$ der Modenkopplungstheorie [Göt92; Rät07b] auf, die den Bereich der unterkühlten Flüssigkeit vom (mikroskopischen) Glaszustand trennt [Fau03]. In der unterkühlten Flüssigkeit ist die Dynamik durch Fließprozesse, also globale Bewegung aller Teilchen, dominiert und man findet eine niedrige Aktivierungsenthalpie für Diffusion, wohingegen im Glaszustand durch die 
höhere Dichte lokale Umordnungen mit deutlich höherer Aktivierungsenthalpie für die Bewegung verantwortlich sind, da die eine Kette umgebende Matrix auf der Zeitskala der Bewegung einzelner Teilchen fest erscheint. Dabei gibt es qualitativ keine Unterschiede zwischen Oberflächen- und Volumenbereichen, man findet für alle Arrhenius-Verhalten ober- und unterhalb von einer gemeinsamen Übergangstemperatur $T_{D}^{*}$ und ein Abknicken des Verlaufs in dieselbe Richtung. Das ist wiederum ein Hinweis darauf, dass sich Oberfläche und Volumen nicht in zwei verschiedene Phasen trennen. Dennoch sind die Aktivierungsenthalpien an den Oberflächen stets niedriger als im Volumen. Dieser Unterschied ist im Glaszustand größer als in der unterkühlten Flüssigkeit, d. h. also, dass das Einfrieren der Dynamik während des Abkühlens im Volumen stärker als an den Oberflächen ist.

\subsubsection{Einfluss der Oberflächen auf die konformative Dynamik}

Die Untersuchung der Dynamik mit Hilfe von effektiven Diffusionskonstanten bietet nur Zugang zum Langzeitverhalten der Dynamik. Um die Unterschiede zwischen der Dynamik im Oberflächenbereich und im Volumen weiter herauszuarbeiten, sind in Abs. 8.3.2 die Fluktuationen des Gyrationsradius betrachtet und in Abhängigkeit sowohl von der Temperatur als auch von der Beobachtungszeit untersucht worden. Beide sind entscheidend dafür, ob ein System glasartig erscheint oder nicht [Zar91]. Wie bereits in Abs. 8.3.2 erwähnt, sind Änderungen des Gyrationsradius und damit dessen Fluktuationen mit Änderungen der Konformation und folglich mit struktureller, bzw. konformativer Relaxationen verbunden, zumindest dann, wenn die Fluktuationen nicht zu klein - also zumindest größer als thermische Fluktuationen - sind. Die Tiefenprofile der Fluktuationsstärke zeigen für steigende Temperaturen die gleiche Entwicklung wie für längere Beobachtungszeiträume (vgl. die Abb. 8.10 und 8.11). Dies deutet die Äquivalenz von Beobachtungszeit und Temperatur an, d. h. längeres Beobachten bei einer bestimmten Temperatur hat den gleichen Effekt wie eine kürzere Beobachtungszeit bei einer höheren Temperatur (und umgekehrt), wie es auch bei der Verformung von metallischen Gläsern und deren mechanischer Antwort gefunden worden ist [Zin06].

Die Fluktuationen sind bei kurzen Beobachtungszeiträumen und niedrigen Temperaturen zu den Oberflächen hin viel stärker als im Volumen, aber auch hier ist der Übergang wie bei der diffusiven Dynamik glatt. Mit steigender Temperatur und mit zunehmender Beobachtungszeit gleichen sich die Werte im Oberflächenbereich und im Volumen an. Für genügend große Beobachtungszeiten tastet eine Kette außerdem das gesamte System ab und erreicht so jede Entfernung von der Oberfläche, was sicherlich auch ein Grund für die Angleichung von Oberflächen- und Volumenverhalten ist. Des Weiteren scheint es eine maximale Fluktuationsstärke bei $\Delta R_{G}^{2} \approx 3 \sigma^{2}$ zu geben. Dieses Verhalten kann man verstehen, wenn man die Änderungen der Konformation als Folge von Relaxationsprozessen sieht. Man kann die Fluktuationen als ein Abtasten der möglichen Konfigurationen und damit der möglichen Zustände mit verschiedener Energie betrachten. Sind die Fluktuationen groß genug, werden alle möglichen Zustände innerhalb der Beobachtungszeit erreicht und die Fluktuationsstärke wird nicht weiter anwachsen. Da hier nur Ketten aus 30 Teilchen betrachtet werden, deren mittlerer Gyrationsradius $\left\langle R_{G}^{2}\right\rangle \approx 7 \sigma^{2}$ beträgt, ist eine Fluktuationsstärke von $\left\langle\Delta R_{G}^{2}\right\rangle \approx 3 \sigma^{2}$ sicherlich groß genug, um alle möglichen Zustände zu erreichen. Der Zeitraum, in dem diese maxima- 
le Fluktuationsstärke erreicht wird, hängt dabei von der Temperatur ab. Der flüssige Zustand oberhalb der kritischen Temperatur der Modenkopplungstheorie erlaubt dabei eine vollständige Abtastung, während der Zeitraum für tiefere Temperaturen sukzessive zunimmt, bis ein vollständiges Abtasten innerhalb des Beobachtungszeitraums im Glaszustand $\left(T<T_{g}\right)$ nicht mehr möglich ist. Da sich der Gyrationsradius nur leicht mit der Temperatur ändert, ist die maximale Fluktuationsstärke hauptsächlich von der Kettenlänge beeinflusst.

Im Bild einer potentiellen Energielandschaft (PEL) (vgl. Abs. 2.1.1 und [Deb01]) entsprechen kleine Änderungen der Konformation, also kleine Fluktuationen, lokalen Schwingungen innerhalb eines lokalen Minimums der PEL. Übergänge zwischen Megabasins sind dagegen nur bei großen Fluktuationsstärken möglich, welche gleichbedeutend mit großen Konformationsänderungen vieler Ketten sind. Bei mittleren Fluktuationsamplituden gibt es Übergänge zwischen benachbarten Minima. Wenn innerhalb eines kurzen Zeitraums viele dieser mittleren Änderungen auftreten, kann es ebenfalls zu einem Übergang zwischen Megabasins kommen. Somit beschreibt die zeitliche Entwicklung der Fluktuationen gewissermaßen die strukturelle Entwicklung innerhalb der potentiellen Energielandschaft. Diese zeitliche Entwicklung, die in Abb. 8.12 für einige Temperaturen für den Oberflächenbereich und für das Volumen aufgetragen ist, zeigt bei allen Temperaturen ein Ansteigen bis zum Maximalwert von ungefähr $3 \sigma^{2}$, der allerdings bei den niedrigsten Temperaturen wie oben beschrieben innerhalb der Beobachtungszeit nicht erreicht wird. Der Zeitraum dieses Anwachsens nimmt mit zunehmender Temperatur deutlich ab. Zur besseren Übersicht sind in dieser Abbildung nur einige Temperaturen gezeigt, um den Trend zu verdeutlichen. Die Werte bei höheren Temperaturen verlaufen alle nahe der $T=0,62 \epsilon / \mathrm{k}_{\mathrm{B}}$ Linie.

Das Anwachsen der mittleren Fluktuationsstärke mit der Zeit kann für alle Temperaturen mit einer gestreckten Exponentialfunktion (nach Kohlrausch [Koh54]) der Form

$$
\left\langle\Delta R_{G}^{2}\right\rangle / A=\left(1-\exp \left(-\left(t / \tau_{r}\right)^{\beta}\right)\right)
$$

mit einem Exponenten $\beta \approx 0,44(5)$, der maximalen Fluktuationsstärke $A$ und einer charakteristischen Relaxationszeit $\tau_{r}=\tau_{r}(T)$ angepasst werden. In Abb. 9.7 sind diese Anpassungen zusammen mit den Daten aus Abb. 8.12 gezeigt. Ein sehr ähnliches Verhalten ist für zeitliche Fluktuationen des Elastizitätstensors im Bereich der Glasübergangstemperatur in metallischen Gläsern gefunden und auf die Kinetik der Abtastung des Phasenraums zurückgeführt worden [May09]. Dabei ist der Anteil an noch nicht besuchten Konfigurationen - oder lokalen Minima in der PEL - zur Zeit $t$ gerade durch $1-\exp \left(-\left(t / \tau_{r}\right)^{\beta}\right)$ gegeben, wobei man für eine Zufallsbewegung auf einer gleichmäßig verteilten PEL $\beta=0,5$ findet [May09]. Für ungleichmäßige Verteilungen der Minima in der PEL, wie man sie für komplexe Systeme erwarten kann, kann dieser Wert abweichen. Da die Fluktuationsstärke ein Maß für Konfigurationsänderungen ist, wird ihr Verlauf (normiert auf die maximale Fluktuationsstärke) durch die gleiche gestreckte Exponentialfunktion beschrieben.

Aus den Anpassungen nach Gl. (9.4) kann man zu jeder Temperatur die charakteristische Relaxationszeit für Oberflächen- und Volumenbereiche bestimmen. Diese Temperaturabhängigkeit ist in Abb. 9.8 dargestellt. Sie ist sehr ähnlich zu den Ergebnissen der effektiven Diffusionskonstanten in Abb. 9.6. Sowohl für die Oberflächen als auch 


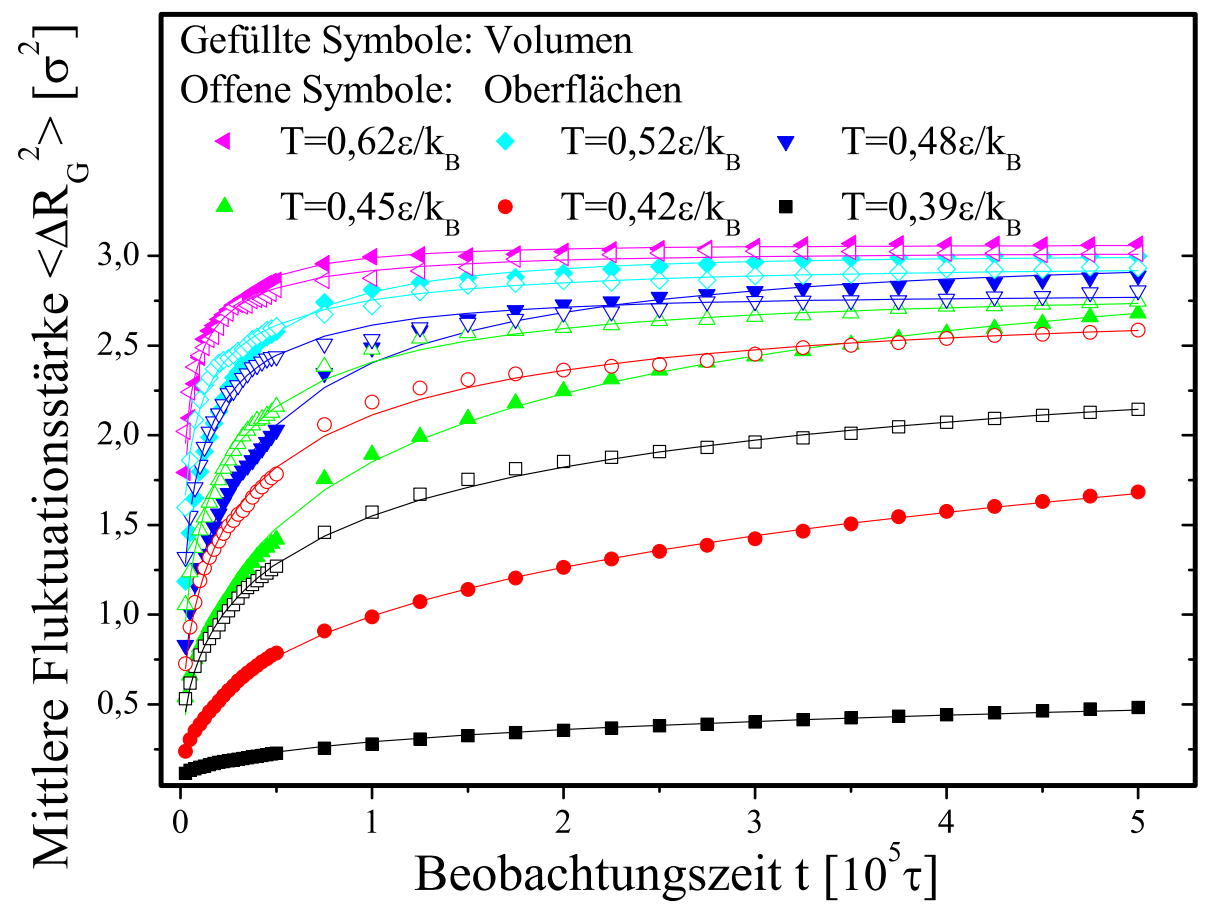

Abbildung 9.7: Entwicklung der Fluktuationsstärke des Gyrationsradius mit der Länge des Beobachtungszeitraums für verschiedene Temperaturen (Symbole) mit der Anpassung einer gestreckten Exponentialfunktion nach Gl. (9.4) (Linien). Der Exponent der Streckung in der Anpassung liegt bei $\beta \approx$ $0,44(5)$.

für das Volumen kann man klar zwei Bereiche voneinander trennen, die ober- und unterhalb einer kritischen Temperatur $T_{R}^{*} \approx 0,47 \epsilon / \mathrm{k}_{\mathrm{B}}$ liegen. In beiden Bereichen liegt für Oberflächen- und Volumenbereiche Arrhenius-Verhalten mit entsprechenden Aktivierungsenthalpien $\Delta H_{R}$ für die Konformationsänderungen vor, die in Abb. 9.8 eingetragen sind. Bei niedrigen Temperaturen findet man $\Delta H_{R} \approx 4,9(4) \epsilon$ im Volumen und $\Delta H_{R} \approx 2,2(2) \epsilon$ im Oberflächenbereich, wohingegen die Werte bei hohen Temperaturen bei $\Delta H_{R} \approx 2,0(1) \epsilon$ im Volumen und $\Delta H_{R} \approx 1,6(1) \epsilon$ im Oberflächenbereich liegen. $\Delta H_{R}$ ist also an den Oberflächen stets niedriger als die entsprechenden Werte im Volumen, was aufgrund der geringeren Behinderungen durch die Umgebung auch verständlich ist. Das Auftreten einer kritischen Temperatur $T_{R}^{*}$, die sehr nahe bei $T_{D}^{*}$ liegt, kann man wiederum verstehen, wenn man $T_{R}^{*}$ mit der kritischen Temperatur $T_{c}$ der Modenkopplungstheorie in Verbindung setzt. Oberhalb von $T_{c}$ erscheint die eine Kette umgebende Matrix flüssig auf der Zeitskala der molekularen Dynamik und behindert somit die Konformationsänderung der Kette weniger, was sich in einer reduzierten Aktivierungsenthalpie im Vergleich zum Glaszustand mit eingefrorener Umgebung („Cage-effect" [Göt92]) unterhalb von $T_{c}$ äußert. 


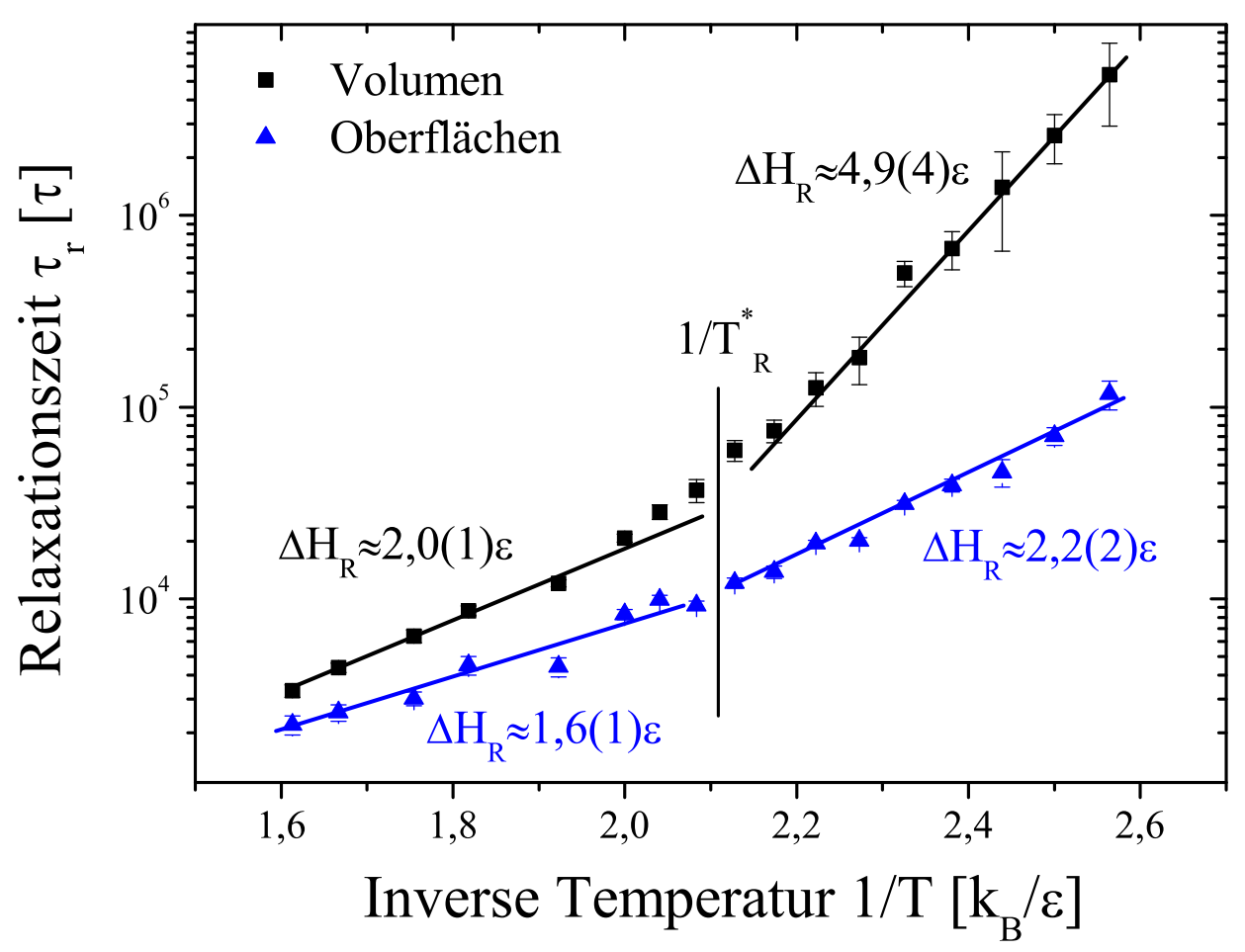

Abbildung 9.8: Arrhenius-Auftragung der charakteristischen Relaxationszeit $\tau_{r}$ für den Oberflächenbereich (blaue Dreiecke) und den Volumenbereich (schwarze Quadrate). In beiden Bereichen liegen die Werte sowohl im flüssigkeitsähnlichen Regime bei hohen Temperaturen als auch im Glas bei niedrigen Temperaturen auf Geraden mit unterschiedlicher Steigung, wobei die Steigungen im Glaszustand deutlich höher sind. Die aus den Steigungen bei hohen und bei niedrigen Temperaturen bestimmten Aktivierungsenthalpien für die unterschiedlichen Bereiche sind mit aufgeführt. Der flüssigkeitsähnliche Bereich bei hohen Temperaturen ist im Volumen und an den Oberflächen klar vom glasartigen Zustand durch veränderte Aktivierungsenthalpien getrennt. Der Übergang erfolgt bei der Temperatur $T_{R}^{*} \approx 0,47 \epsilon / \mathrm{k}_{\mathrm{B}}$. 


\subsubsection{Kopplung von diffusiver und konformativer Dynamik}

Ein Vergleich der diffusiven Dynamik und der strukturellen Rekonfiguration aus den beiden vorangegangenen Abschnitten führt zu der Schlussfolgerung, dass beiden dieselben molekularen Relaxationsprozesse zugrundeliegen und zwar sowohl im flüssigen Zustand als auch Glaszustand. Die zugehörigen Arrhenius-Auftragungen der Relaxationszeiten ${ }^{4}$ in den Abb. 9.6 und 9.8 liefern, wie bereits beschrieben, identische Aktivierungsenthalpien über und unter $T_{c}$ im Oberflächen- und im Volumenbereich. Es gilt also $\Delta H_{D}=\Delta H_{R}$ und $T_{D}^{*}=T_{R}^{*}$ für alle Bereiche. Der Zusammenhang verschiedener Relaxationsprozesse in Gläsern wird aktuell auch bei der Interpretation experimenteller Daten diskutiert [Ric07; Hac08] und gehört außerdem zu den Grundlagen der Modenkopplungstheorie [Göt92]. Im flüssigen Zustand oberhalb von $T_{c}$ ist ein ähnlicher Zusammenhang zwischen Diffusionskonstante und der Orientierungsrelaxationszeit ${ }^{5}$ des End-zu-End Abstands, der eine ähnliche Längenskala wie der Gyrationsradius hat, gefunden worden [Bas05]. Die Abklingzeit der inkohärenten Streufunktion, die als strukturelle Relaxationszeit auf der Längenskala eines Monomers interpretiert werden kann, zeigt allerdings eine andere Temperaturabhängigkeit.

Zusammenfassend heißst das, dass Diffusion und konformative Relaxation in allen Bereichen gekoppelt sind und auf denselben molekularen Relaxationsprozessen beruhen. Das kann man so interpretieren, dass einzelne Ketten durch zufällige Bewegung in der potentiellen Energielandschaft, die eine Projektion des Konformationsraums darstellt, ihre möglichen Konformationen abtasten, wie es durch $\beta \approx 0,5$ wie in [May09] bestätigt wird. Dabei ist jede Konformationsänderung mit einer zufälligen Bewegung im Realraum verbunden. Diese Art der Kettenbewegung ist eine der Grundlagen des Reptationsmodells [Gen71].

\footnotetext{
${ }^{4}$ Die intrinsische Relaxationszeitskala der Diffusion ist $\propto 1 / D$, damit kann man aus den effektiven Diffusionskonstanten in Abb. 9.6 die Relaxationszeit der Diffusion bestimmen.

${ }^{5}$ Im Englischen orientational relaxation time.
} 


\section{Zusammenfassung}

In der vorliegenden Arbeit ist die Dynamik von dünnen Polymerfilmen zum einen experimentell zusammen mit Kontinuumsmodellierungen und zum anderen mit Molekulardynamik Simulationen untersucht worden. Dazu wurden dünne Polycarbonat (PC) Filme durch thermisches Verdampfen bei $300{ }^{\circ} \mathrm{C}$ im Vakuum auf verschiedenen Substraten (Si, $\mathrm{SiO}_{2}, \mathrm{GaAs}$ und PC) hergestellt, experimentell charakterisiert und mit stochastischen Ratengleichungen modelliert. Zum anderen wurde die Dynamik von dünnen, freistehenden Polymerfilmen mithilfe von Molekulardynamik Simulationen betrachtet. Des Weiteren wurden erste Tests zur Messung lokaler mechanischer Eigenschaften durchgeführt. Dafür wurden einerseits Kraft-Abstandskurven ausgewertet und andererseits das vorhandene Rasterkraftmikroskop zu einem akustischen Rasterkraftmikroskop (AFAM) erweitert. Bei beiden Methoden müssen aber auf den Polymerschichten noch Probleme der plastischen Verformung gelöst werden.

Es konnte durch Fourier-Transformations-Infrarotspektroskopie gezeigt werden, dass die Bindungsstruktur des Polycarbonats, also vor allem der Aufbau des Monomers, durch das thermische Verdampfen nicht verändert wird, insbesondere kann Dekomposition, also die vollständige Zersetzung des Polymers, ausgeschlossen werden. Durch Messungen der Molmassen und damit der Kettenlängen mit Größenausschlusschromatographie und Massenspektrometrie konnten als Hauptbestandteile der aufgedampften Schichten oligomeres Polycarbonat, also kurzkettiges Material, das nur aus wenigen Monomeren besteht, identifiziert werden. Dazu wurde noch ein kleiner Anteil mit hoher Molmasse beobachtet. Die kürzeren Ketten werden durch thermische Degradation gebildet. Dabei wird eine Kette an statistisch verteilten schwächeren Bindungen gespalten. Die Abnahme dieser schwächeren Bindungen mit der Zeit folgt einer Kinetik 1. Ordnung. Diese Abnahme sorgt dafür, dass sich die Aufdampfrate mit zunehmender Zeit verringert, da die Produktion der verdampfbaren kürzeren Ketten der limitierende Schritt wird. Aus der Temperaturabhängigkeit der anfänglichen Aufdampfrate wurde die Verdampfungsenthalpie zu $\Delta H \approx 125 \mathrm{~kJ} / \mathrm{mol}$ bestimmt. Die PC Schichten sind den Weitwinkelröntgenmessungen nach amorph, es gibt keine Hinweise auf Kristallisation.

Untersuchungen der Topographie auf den unterschiedlichen Substraten in Abhängigkeit von der Schichtdicke mittels Rasterkraftmikroskopie haben gezeigt, dass sich das Wachstumsverhalten auf PC Substraten stark von dem auf den anderen - anorganischen - Substraten unterscheidet. Außerdem muss man bei den Schichten auf den anorganischen Substraten zwischen den anfänglichen Strukturen und ihrer zeitlichen Entwicklung unterscheiden.

Für PC auf PC ist keine ausgezeichnete Struktur oder Schichtdickenabhängigkeit zu erkennen. Die Oberflächen sind für alle untersuchten Schichtdicken glatt und homogen, die Rauigkeit liegt etwa bei $\sigma \approx 0,5(2) \mathrm{nm}$. Die Schichten bleiben über den gesamten untersuchten Zeitraum stabil und verändern sich nicht mehr im Laufe der Zeit. Insbesondere ist kein Anstieg der Rauigkeit zu sehr dünnen Schichten hin zu erkennen. 


\section{Zusammenfassung}

Eine stark erhöhte Rauigkeit bei sehr dünnen Schichten zeichnet die Rauigkeitsentwicklung auf $\mathrm{Si}, \mathrm{SiO}_{2}$ und GaAs aus. Auf diesen Substraten findet man bei dünneren Filmen eine ausgeprägte Struktur mit grabenartigen und runden Vertiefungen, die große Ähnlichkeit mit der Struktur von entnetzenden Polymerfilmen (vor allem Polystyrol auf $\mathrm{SiO}_{2}$ ) haben. Dickere Filme mit $d \gtrsim 50 \mathrm{~nm}$ zeigen diese Vertiefungen nicht mehr, sondern bilden homogene, glatte und geschlossene Schichten. Außerdem hat sich gezeigt, dass die dünnen Filme auf den anorganischen Substraten bei Raumtemperatur nicht stabil sind, sondern sehr langsam über einen Zeitraum von mehreren Wochen entnetzen, also immer mehr Substratfläche freilegen und sich zu Tropfen zusammenziehen. Die dickeren Filme verändern sich dagegen über den gesamten beobachteten Zeitraum von einigen Monaten nicht. Daraus folgt, dass es sich bei PC auf den untersuchten anorganischen Substraten um metastabile Systeme handelt, bei denen dicke Schichten stabil, aber dünne Schichten instabil, d. h. auf diesen Substraten nicht benetzend sind. Diese Entnetzung im Laufe der Zeit bei Raumtemperatur kann man durch eine Reduzierung der Glasübergangstemperatur aufgrund der niedrigeren Molmasse und der geringeren Schichtdicke erklären. Beruhend auf Literaturdaten haben Abschätzungen zum Einfluss der Molmasse und der Schichtdicke gezeigt, dass die Glasübergangstemperatur bis in die Nähe der Raumtemperatur abgesenkt sein kann.

Um die Wachstumsprozesse, die zu den anfänglichen Strukturen führen, identifizieren zu können, wurde ein minimales Kontinuumsmodell vorgestellt, das die wesentliche Strukturbildung während des Aufdampfens reproduziert. Das Modell beinhaltet neben der Deposition nur die durch das chemische Potential getriebene Oberflächendiffusion. Das chemische Potential ist aus Beiträgen aus der Oberflächenkrümmung und dem effektiven Grenzflächenpotential, dessen wesentlicher Parameter die Hamaker-Konstante ist, zusammengesetzt. Je nach Wahl des Vorzeichens der Hamaker-Konstante kann ein stabiles System $(A<0)$ oder ein instabiles System $(A>0)$ modelliert werden. Durch eine geeignete Parametrisierung und Reskalierung der numerischen Lösung der Kontinuumsgleichung konnte eine qualitative und quantitative Übereinstimmung mit dem Wachstum von PC auf GaAs erzielt werden. Es konnte also gezeigt werden, dass die anfänglichen Strukturen allein durch Oberflächendiffusion und statistische Deposition gebildet werden können.

Das thermische Aufdampfen von PC bietet interessante Anwendungsmöglichkeiten für zukünftige Strukturbildungsexperimente, da sich durch die Wahl der Schichtdicke und der Substrattemperatur unterschiedliche Strukturen einstellen lassen. So ist z. B. eine Erweiterung mit einem zweiten Verdampfer für metallische Schichten denkbar, um strukturierte Metallfilme oder auch Metall-Polymer-Multilagen herzustellen. Zukünftige Erweiterungen der Kontinuumsmodellierung könnten Korrekturen höherer Ordnung oder eine Erweiterung auf andere Materialsysteme beinhalten. Außerdem könnte man versuchen, das Langzeitverhalten oder Strukturbildung bei höheren Temperaturen einzubauen.

Bei der Betrachtung der Dynamik von freistehenden, dünnen Polymerfilmen mit Molekulardynamik Simulationen wurde der Einfluss von freien Oberflächen auf die Diffusion und auf konformative Relaxation untersucht. Beide Prozesse sind an den Oberflächen im Vergleich zum Volumen erhöht, wobei der Grad der Erhöhung bei der Diffusion mit zunehmender Temperatur und bei der konformativen Dynamik mit zunehmender Zeit und steigender Temperatur niedriger wird. Durch Arrhenius-Auftragungen wurde für 
beide Arten der Dynamik ein Übergang bei einer kritischen Temperatur gefunden, die im Zusammenhang mit der kritischen Temperatur der Modenkopplungstheorie diskutiert worden ist. Diese Übergangstemperatur ist für Volumen und Oberflächen dieselbe. Die aus den Arrhenius-Auftragungen bestimmten Aktivierungsenthalpien für die diffusive und für die konformative Dynamik stimmen innerhalb des Fehlers sowohl oberhalb als auch unterhalb der Übergangstemperatur, für Volumen und Oberflächen, überein, und daher folgt die Schlussfolgerung, dass beide auf denselben molekularen Relaxationsprozessen beruhen. Die Relaxationen wurden im Bild einer potentiellen Energielandschaft diskutiert und mit einer zufälligen Bewegung einer Kette in ihrem Konformationsraum erklärt. 



\section{Anhang}

\subsection{Hochauflösende Massenspektren}

Das in Abb. 6.4 gezeigte ESI Massenspektrum von thermisch aufgedampftem PC gibt eine gute Übersicht der vorkommenden Massen. Zusätzliche hochaufösende Massenspektren, die in Abb. 11.1 gezeigt sind ${ }^{1}$, gemessen mit einem FT-ICR-MS, erlauben darüber hinaus die Bestimmung der molekularen Zusammensetzung der einzelnen Maxima, wie sie in der Abbildung eingetragen sind. Ein weiterer Vorteil ist, dass das Hintergrundrauschen deutlich geringer als bei dem ESI Massenspektrometer ist.

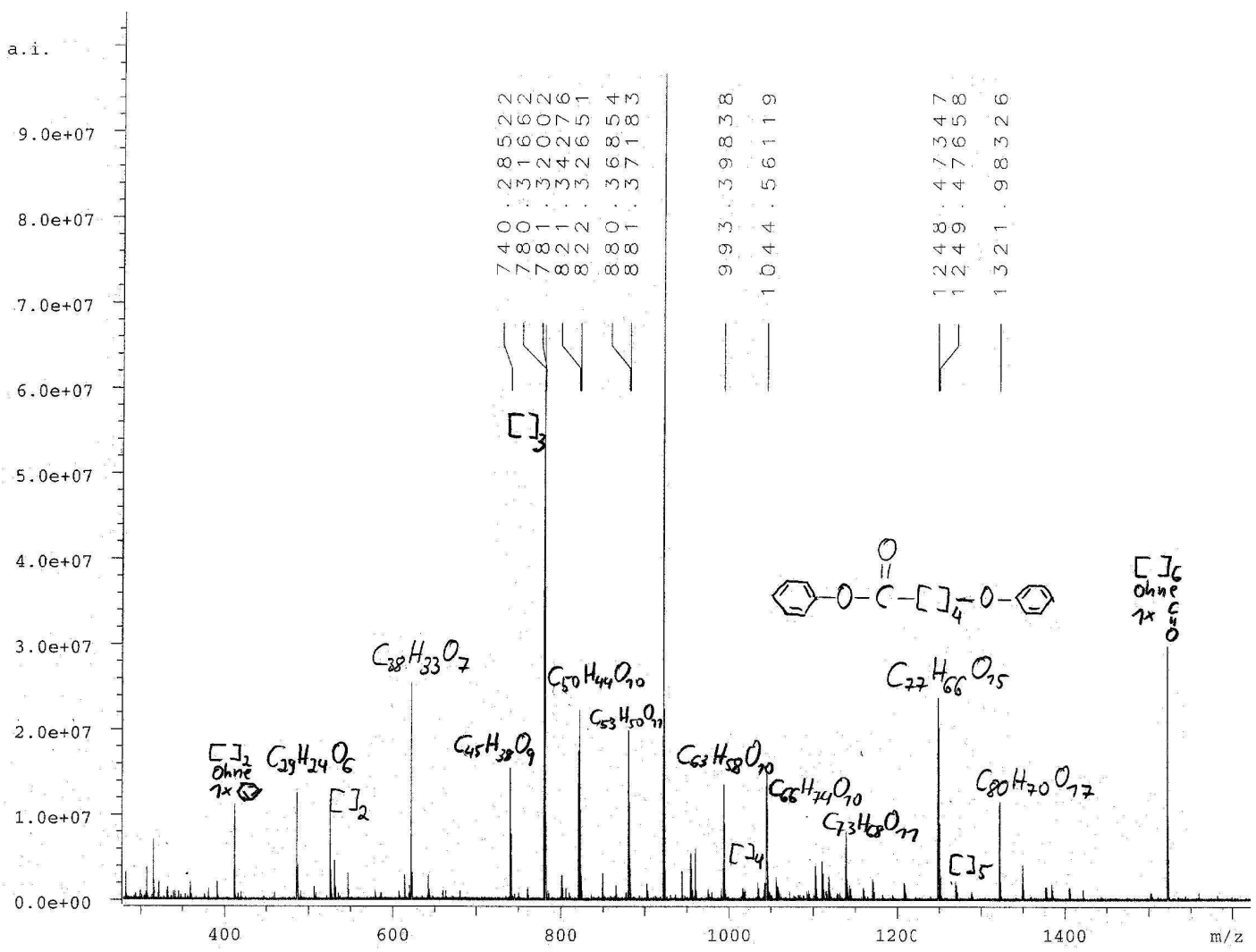

Abbildung 11.1: Hochaufösendes Massenspektrum von thermisch aufgedampftem PC aufgenommen mit einem FT-ICR-MS (vgl. Abs. 3.2.2). Die hohe Auflösung erlaubt eine genaue chemische Zuordnung der Maxima, die in Zusammenarbeit mit F. Günzler (aus der Arbeitsgruppe von Prof. M. Buback am Institut für Physikalische Chemie, Universität Göttingen) vorgenommen worden ist.

\footnotetext{
${ }^{1}$ Die Spektren liegen nicht in digitaler Form vor.
} 


\subsection{Exemplarische Kleinwinkelröntgenmessung}

Für die Kalibrierung der Schwingquarzwaage werden Kleinwinkelröntgenmessungen, wie z. B. in Abb. 11.2 gezeigt, verwendet. Aus den Oszillationen lässt sich mit Gl. (3.1) die Schichtdicke berechnen. Diese Methode vernachlässigt die Brechung der Röntgenstrahlung an der Grenzfläche, also den Einfluss des Brechungsindex. Um dies zu berücksichtigen, kann man die Winkelabhängigkeit der Reflektivität z. B. mit Hilfe des ParratFormalismus zur Bestimmung von Schichtdicke und Rauigkeit anpassen [Par54]. Da hier aber die Stöchiometrie der Filme nicht bekannt ist und die Rauigkeiten mit dem AFM ausgewertet werden, wird auf eine solche Anpassung verzichtet und die vereinfachte Auswertung unter Verwendung von Gl. (3.1) verwendet.

Für die nominell $75 \mathrm{~nm}$ dicke PC Schicht auf Si aus Abb. 11.2 erhält man so die Dicke $d=70(4) \mathrm{nm}$. Der Unterschied und damit der Fehler der Anzeige der Schwingquarzwaage beträgt weniger als $7 \%$.

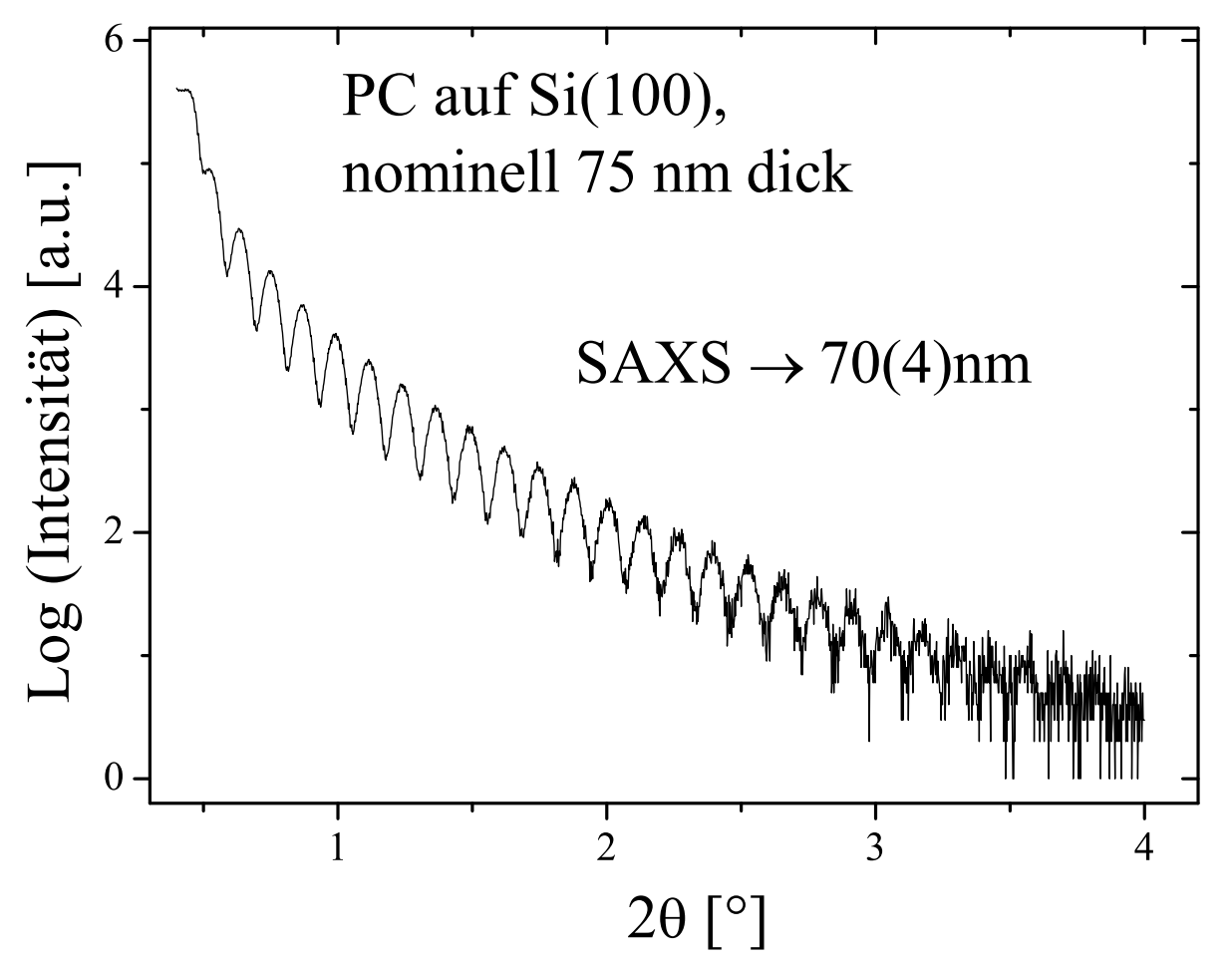

Abbildung 11.2: Exemplarische Kleinwinkelröntgenmessung an einer laut Schwingquarzwaage $75 \mathrm{~nm}$ dicken PC Schicht auf Si. Aus dem Abstand der periodischen Oszillationen wird mit der Bragg-Bedingung die Schichtdicke zu 70(4) nm bestimmt. 


\subsection{Thermisches Verdampfen von Polypropylen und Polystyrol}

Neben Polycarbonat sind testweise auch Polypropylen (PP) bei $T=250{ }^{\circ} \mathrm{C}$ und Polystyrol (PS) bei $T=280{ }^{\circ} \mathrm{C}$ sowie PC bei einer höheren Temperatur von $T=400{ }^{\circ} \mathrm{C}$ thermisch verdampft worden. Bei der Verdampfung PP und PS ist eine starke Blasenbildung zu beobachten und die Flüssigkeit im Tiegel siedet stark, wie es aus früheren Untersuchungen bereits bekannt ist [Ash78]. Abb. 11.3 zeigt die zugehörigen AFM Bilder und eine REM Aufnahme des PS Films. Keine so hergestellte Schicht zeigt einen zusammenhängenden Film, stattdessen findet man separierte Inseln, wie sie bei entnetzenden Filmen zu beobachten sind. Bei der Messung von PP bei Raumtemperatur ergibt sich ein weiteres Problem, da die Glasübergangstemperatur bei $T_{g} \approx-10^{\circ} \mathrm{C}$ liegt und das Material bei der Messung flüssigkeitsähnlich ist. Wie man an den REM Aufnahmen in Abb. 11.3(c) anhand der dendritischen Strukturen sieht, neigen die PS Schichten zur Kristallisation an Defekten oder Verunreinigungen.
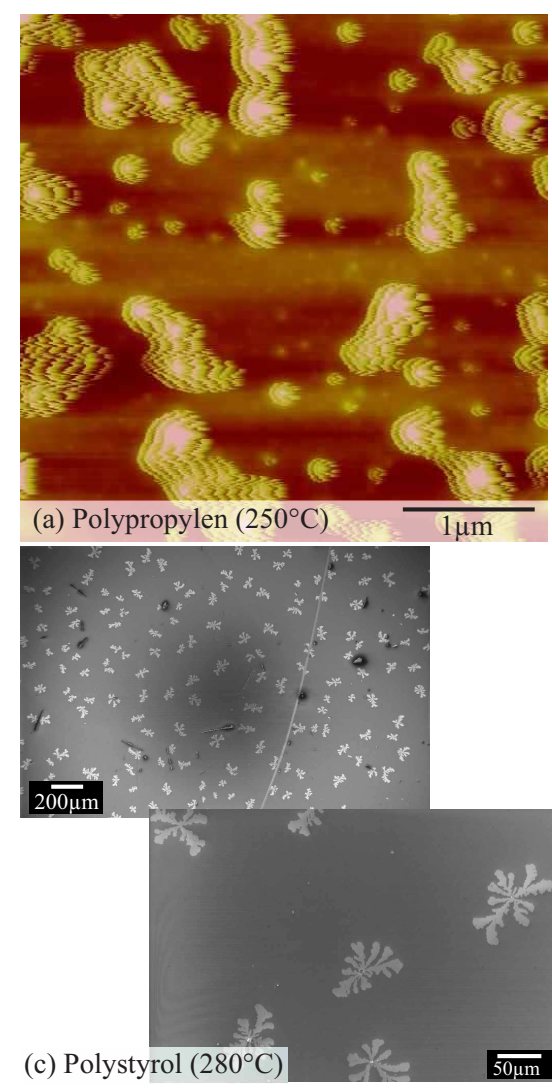

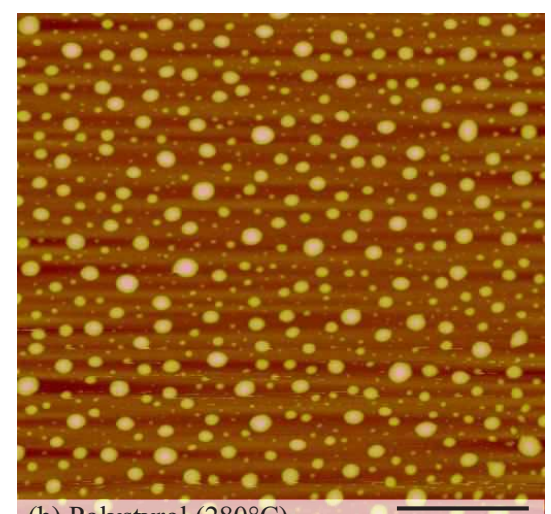

(b) Polystyrol $\left(280^{\circ} \mathrm{C}\right)$

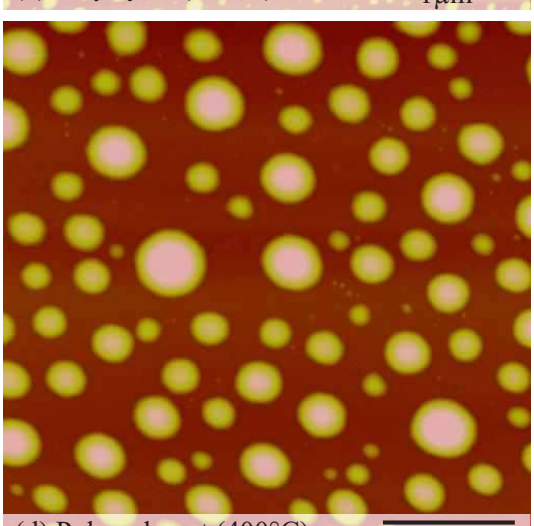

(d) Polycarbonat $\left(400^{\circ} \mathrm{C}\right)$

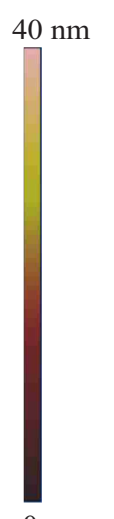

$0 \mathrm{~nm}$

$80 \mathrm{~nm}$

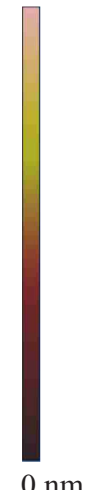

Abbildung 11.3: Exemplarische Topographien von thermisch verdampften Polymeren: (a) PP hergestellt bei $T=250{ }^{\circ} \mathrm{C}$ und (b) PS hergestellt bei $T=280^{\circ} \mathrm{C}$. Statt eines Films bilden sich separierte tropfenartige Inseln. (c) REM Übersichtsbilder verdeutlichen, dass die PS Filme zur Kristallisation an Defekten oder Verunreinigungen neigen. (d) Auch beim Verdampfen von PC bei $T=400{ }^{\circ} \mathrm{C}$ bilden sich separierte tropfenartige Inseln. 


\subsection{Vergleich der Herstellungsvarianten in der MD Simulation}

Die Simulationszellen der MD Untersuchungen werden mit zwei verschiedenen Varianten hergestellt (vgl. Abs. 5.2.2). Während in Variante 1 einige Ketten zerschnitten werden müssen, haben in Variante 2 alle Ketten dieselbe Länge. Um die Ergebnisse vergleichen zu können, werden in Variante 1 nur nicht zerschnittene Ketten in die Auswertung mit einbezogen.

In Abb. 11.4 sind für verschiedene Temperaturen Tiefenprofile der effektiven Diffusionskonstante aufgetragen. Für beide Herstellungsverfahren erhält man qualitativ und quantitativ gleiche, bzw. sehr ähnliche Werte. Ein Unterschied besteht in der Ausdehnung der Zelle in z Richtung. Die nach Variante 1 hergestellten Zellen sind auch aufgrund der fehlenden Teilchen etwas kleiner.

Ein Vergleich der Fluktuationen des Gyrationsradius, der in Abb. 11.5 gezeigt ist, bestätigt, dass die Unterschiede durch die Herstellungsverfahren klein sind.

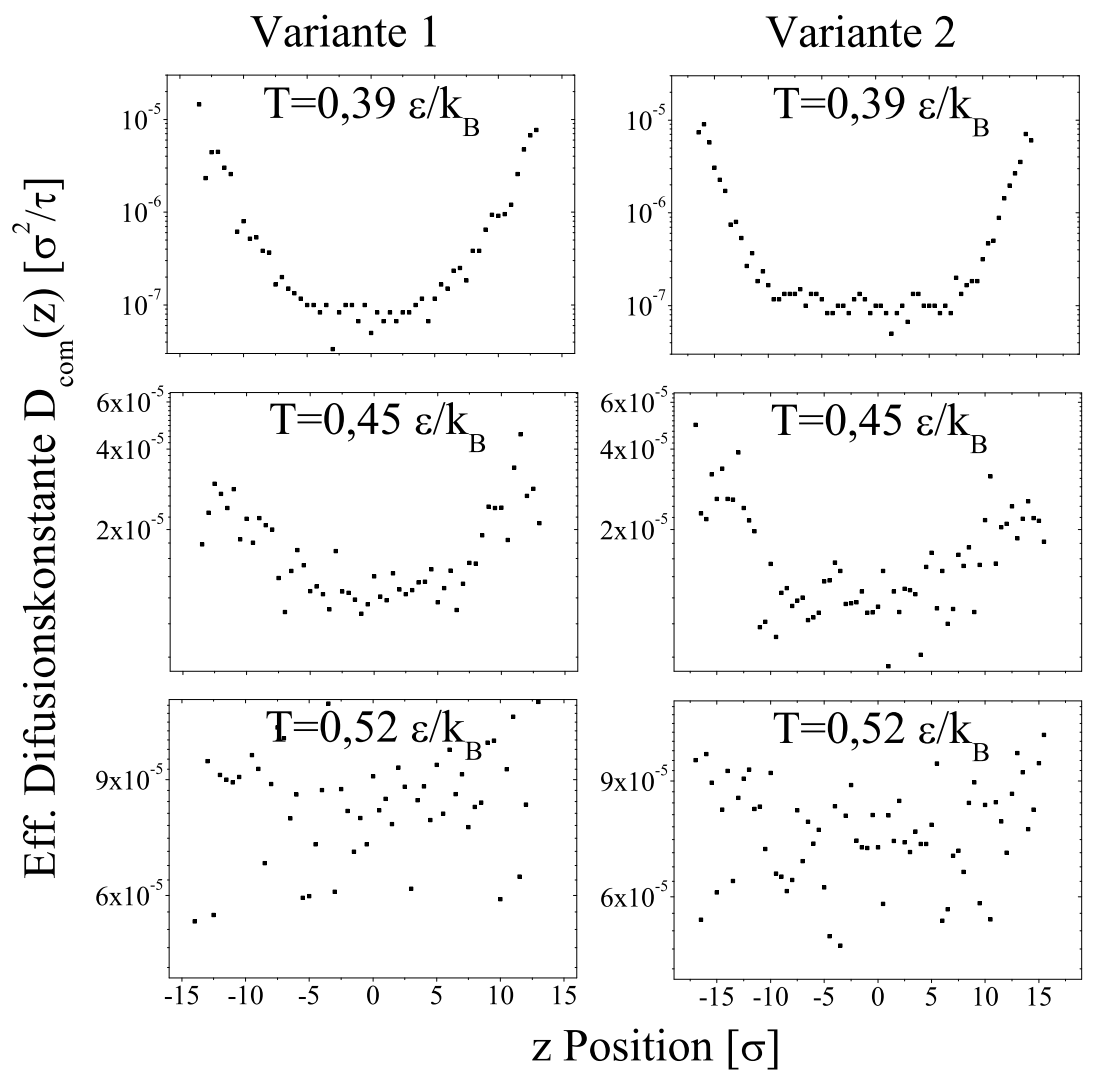

Abbildung 11.4: Vergleich der Tiefenprofile der effektiven Diffusionskonstante $D_{\text {com }}(z)$ auf einer logarithmischen Skala bei verschiedenen Temperaturen für Simulationszellen, die nach Variante 1 (links), bzw. 2 (rechts) hergestellt worden sind. Für Variante 2 sind dieselben Daten wie in Abb. 8.8 aufgetragen. Qualitativ und quantitativ sind die Tiefenprofile für beide Herstellungsvarianten sehr ähnlich. 

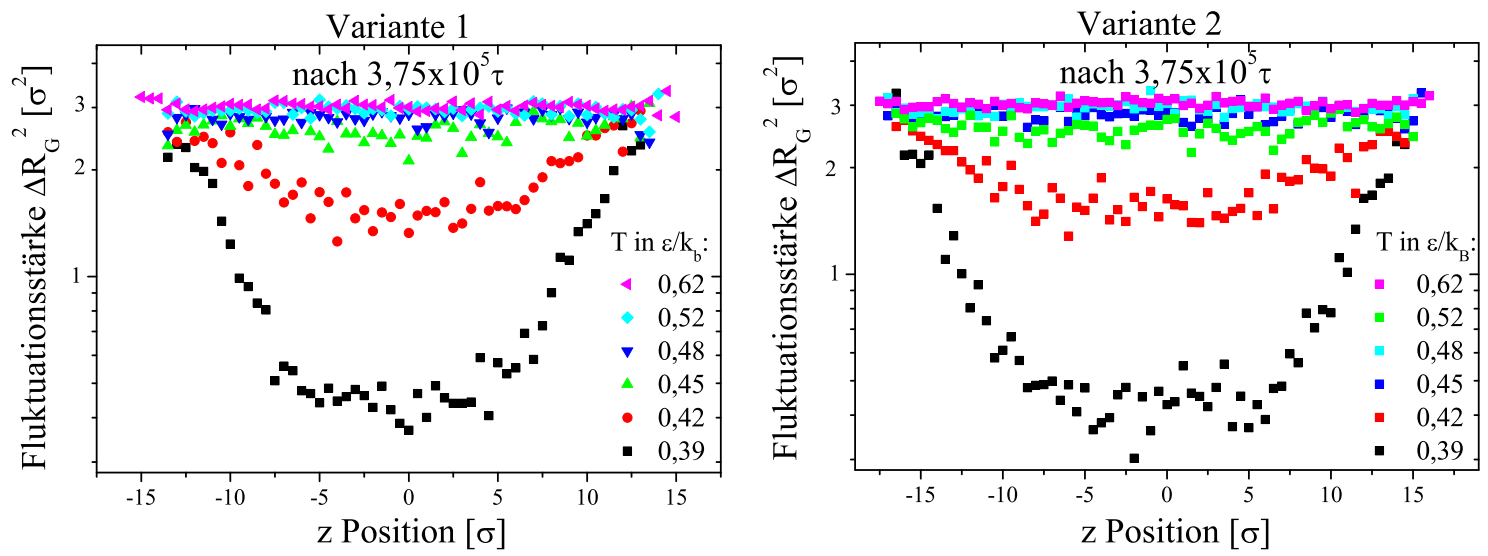

Abbildung 11.5: Vergleich der Fluktuationen des Gyrationsradius bei verschiedenen Temperaturen für Simulationszellen, die nach Variante 1 (links), bzw. 2 (rechts) hergestellt worden sind. Qualitativ und quantitativ sind beide in guter Übereinstimmung. 



\section{Literaturverzeichnis}

[Abr03] Abrams, C. F. und Kremer, K. Combined coarse-grained and atomistic simulation of liquid bisphenol A-polycarbonate: Liquid packing and intramolecular structure. Macromolecules 36, 260 (2003).

[Ada65] Adam, G. und Gibbs, J. H. On the Temperature Dependence of Cooperative Relaxation Properties in Glass-Forming Liquids. J. Chem. Phys. 43, 139 (1965).

[Ada76] Adam, G. A., Hay, J. N., Pasons, I. W. und Haward, R. N. Effect of molecular weight on the thermal properties of polycarbonates. Polymer 17, 51 (1976).

[AH98] A-Hassan, E., Heinz, W. F., Antonik, M. D., D'Costa, N. P., Nageswaran, S., Schoenenberger, C. A. und Hoh, J. H. Relative Microelastic Mapping of Living Cells by Atomic Force Microscopy. Biophys. J. 74, 1564 (1998).

[All89] Allen, M. P. und Tildesley, D. J. Computer simulation of liquids. Oxford University Press, USA (1989).

[Ash78] Ashida, M., Ueda, Y. und Watanabe, T. Epitaxial growth of low molecular weight polypropylene from vapor phase. J. Polym. Sci. - Polym. Phys. Ed. 16, 179 (1978).

[Ash01] Ashcroft, N. W. und Mermin, N. D. Festkörperphysik. Oldenbourg Verlag, München, Wien 1. Aufl. (2001).

[Bal96] Ballone, P. und Rubini, S. Roughening Transition of an Amorphous Metal Surface: A Molecular Dynamics Study. Phys. Rev. Lett. 77, 3169 (1996).

[Bal98] Baldo, M., Deutsch, M., Burrows, P., Gossenberger, H., Gerstenberg, M., Ban, V. und Forrest, S. Organic Vapor Phase Deposition. Adv. Mater. 10, 1505 (1998).

[Bar95] Barabási, A.-L. und Stanley, H. E. Fractal Concepts in Surface Growth. Cambridge University Press, Cambridge 1. Aufl. (1995).

[Bas05] Baschnagel, J. und Varnik, F. Computer simulations of supercooled polymer melts in the bulk and in confined geometry. J. Phys. Cond. Mat. 17, 851 (2005). 
[Bec03] Becker, J., Gruen, G., Seemann, R., Mantz, H., Jacobs, K., Mecke, K. R. und Blossey, R. Complex dewetting scenarios captured by thin-film models. Nature Mater. 2, 59 (2003).

[Ben98] Bennemann, C., Paul, W., Binder, K. und Dünweg, B. Molecular-dynamics simulations of the thermal glass transition in polymer melts: $\alpha$-relaxation behavior. Phys. Rev. E 57, 843 (1998).

[Ben99] Bennemann, C., Baschnagel, J., Paul, W. und Binder, K. Molecular-dynamics simulation of a glassy polymer melt: Rouse model and cage effect. Comp. Theor. Polym. Sci. 9, 217 (1999).

[Ben00] Bentrem, F. W., Pandey, R. B. und Family, F. Roughening, deroughening, and nonuniversal scaling of the interface width in electrophoretic deposition of polymer chains. Phys. Rev. E 62, 914 (2000).

[Bin81] Binnig, G., Rohrer, H., Gerber, C. und Weibel, E. Tunneling through a controllable vacuum gap. Appl. Phys. Lett. 40, 178 (1981).

[Bin82] Binnig, G., Rohrer, H., Gerber, C. und Weibel, E. Surface Studies by Scanning Tunneling Microscopy. Phys. Rev. Lett. 49, 57 (1982).

[Bin86] Binnig, G., Quate, C. F. und Gerber, C. Atomic Force Microscopy. Phys. Rev. Lett. 56, 930 (1986).

[Bin04] Binder, K., Varnik, F., Baschnagel, J., Scheidler, P. und Kob, W. Computer Simulation of the Glass Transition in Thin Films. AIP Conf. Proc. 708, 509 (2004).

[Bli02] Bliznyuk, V. N., Assender, H. E. und Briggs, G. A. D. Surface glass transition temperature of amorphous polymers. A new insight with SFM. Macromolecules 35, 6613 (2002).

[Böd99] Böddeker, B. und Teichler, H. Dynamics near free surfaces of molecular dynamics simulated $N i_{0.5} Z r_{0.5}$ metallic glass films. Phys. Rev. E 59, 1948 (1999).

[Bub07] Buback, M., Frauendorf, H., Günzler, F. und Vana, P. Electrospray ionization mass spectrometric end-group analysis of PMMA produced by radical polymerization using diacyl peroxide initiators. Polymer 48, 5590 (2007).

[Cap99] Cappella, B. und Dietler, G. Force-distance curves by atomic force microscopy. Surf. Sci. Rep. 34, 1 (1999).

[Cap08] Cappella, B. und Silbernagl, D. Nanomechanical properties of polymer thin films measured by force-distance curves. Thin Sol. Films 516, 1952 (2008).

[Chi77] Chiang, C. K., Fincher, C. R., Park, Y. W., Heeger, A. J., Shirakawa, H., Louis, E. J., Gau, S. C. und MacDiarmid, A. G. Electrical Conductivity in Doped Polyacetylene. Phys. Rev. Lett. 39, 1098 (1977). 
[Coh59] Cohen, M. H. und Turnbull, D. Molecular transport in liquids and glasses. J. Chem. Phys. 31, 1164 (1959).

[Col94] Collins, G. W., Letts, S. A., Fearon, E. M., McEachern, R. L. und Bernat, T. P. Surface Roughness Scaling of Plasma Polymer Films. Phys. Rev. Lett. 73, 708 (1994).

[Dav64] Davis, A. und Golden, J. H. Degradation of polycarbonates III. Viscometric study of thermally induced chain scission. Die Makromol. Chem. 78, 16 (1964).

[Dav68] Davis, A. und Golden, J. H. Thermal degradation of polycarbonate. J. Chem. Soc. B 1968, 45 (1968).

[Deb01] Debenedetti, P. G. und Stillinger, F. H. Supercooled liquids and the glass transition: Complex systems. Nature 410, 259 (2001).

[Des06] Desai, T. G., Keblinski, P., Kumar, S. K. und Granick, S. Molecular-dynamics simulations of the transport properties of a single polymer chain in two dimensions. J. Chem. Phys. 124, 084904 (2006).

[Des07] Desai, T. G., Keblinski, P., Kumar, S. K. und Granick, S. Modeling Diffusion of Adsorbed Polymer with Explicit Solvent. Phys. Rev. Lett. 98, 218301 (2007).

[Dix90] Dixon, P. K., Wu, L., Nagel, S. R., Williams, B. D. und Carini, J. P. Scaling in the relaxation of supercooled liquids. Phys. Rev. Lett. 65, 1108 (1990).

[Doi86] Doi, M. und Edwards, S. F. The Theory of Polymer Dynamics. Oxford Science Publications, Oxford (1986).

[Edw82] Edwards, S. F. und Wilkinson, D. R. The surface statistics of a granular aggregate. Proc. R. Soc. Lond. A 381, 17 (1982).

[Ell90] Elliott, S. R. Physics of Amorphous Materials. Longman Group and Wiley, Essex, New York 2. Aufl. (1990).

[Fak07] Fakhraai, Z. Dynamics of polymer thin films and surfaces. Phd thesis University of Waterloo (2007).

[Fak08] Fakhraai, Z. und Forrest, J. A. Measuring the Surface Dynamics of Glassy Polymers. Science 319, 600 (2008).

[Fal97] Falbe, J. und Regitz, M. IR-Spektroskopie. In RÖMPP Lexikon: Chemie Bd. 3 H-L. Thieme Verlag (1997).

[Fau03] Faupel, F., Frank, W., Macht, M. P., Mehrer, H., Naundorf, V., Rätzke, K., Schober, H. R., Sharma, S. K. und Teichler, H. Diffusion in metallic glasses and supercooled melts. Rev. Mod. Phys. 75, 237 (2003). 
[For01] Forrest, J. A. und Dalnoki-Veress, K. The glass transition in thin polymer films. Adv. Coll. Inter. Sci. 94, 167 (2001).

[Fox55] Fox, T. G. und Loshaek, S. Influence of Molecular Weight and Degree of Crosslinking on the Specific Volume and Glass Temperature of Polymers. J. Polym. Sci. 15, 371 (1955).

[Gat07] Gates, R. S. und Reitsma, M. G. Precise atomic force microscope cantilever spring constant calibration using a reference cantilver array. Rev. Sci. Instr. 78, 086101 (2007).

[Gef06] Geffroy, B., le Roy, P. und Prat, C. Review: Organic light-emitting diode (OLED) technology: materials, devices and display technologies. Polym. Intern. 55, 572 (2006).

[Gen71] de Gennes, P. G. Reptation of a Polymer Chain in the Presence of Fixed Obstacles. J. Chem. Phys. 55, 572 (1971).

[Gen85] de Gennes, P. G. Wetting: statics and dynamics. Rev. Mod. Phys. 57, 827 (1985).

[Gol64] Golden, J. H., Hammant, B. L. und Hazell, E. A. Degradation of polycarbonates. IV. Effect of molecular weight on flexural properties. J. Polym. Sci. A 2, 4787 (1964).

[Göt92] Götze, W. und Sjögren, L. Relaxation processes in supercooled liquids. Rep. Prog. Phys. 55, 241 (1992).

[Gra03] Granick, S., Kumar, S. K., Amis, E. J., Antonietti, M., Balazs, A. C., Chakraborty, A. K., Grest, G. S., Hawker, C., Janmey, P., Kramer, E. J., Nuzzo, R., Russel, T. P. und Safinya, C. R. Macromolecules at Surfaces: Research Challenges and Opportunities from Tribology to Biology. J. Polym. Sci. B 41, 2755 (2003).

[Gue00] Guerdane, M. Structure and Dynamics of Molecular-Dynamics Simulated Undercooled Ni-Zr-Al Melts. Dissertation Universität Göttingen (2000).

[Hac04] Hachenberg, J., Streng, C., Süske, E., Vauth, S., Mayr, S. G., Krebs, H.U. und Samwer, K. Kinetic Roughening of Laser Deposited Polymer Films: Crossover from Single Particle Character to Continuous Growth. Phys. Rev. Lett. 92, 246102 (2004).

[Hac08] Hachenberg, J., Bedorf, D., Samwer, K., Richert, R., Kahl, A., Demetriou, M. D. und Johnson, W. L. Merging of the $\alpha$ and $\beta$ relaxations and aging via the Johari-Goldstein modes in rapidly quenched metallic glasses. Appl. Phys. Lett. 92, 131911 (2008).

[Han88] Hansen, S. G. und Robtaille, T. E. Formation of polymer films by pulsed laser evaporation. Appl. Phys. Lett. 52, 81 (1988). 
[Hec08] Hecksher, T., Nielsen, A. I., Olsen, N. B. und Dyre, J. C. Little evidence for dynamic divergences in ultraviscous molecular liquids. Nature Phys. 4, 737 (2008).

[Her81] Hertz, H. Über die Berührung fester elastischer Körper. J. Reine Angew. Math. 92, 156 (1881).

[Her50] Herring, C. Effect of Change of Scale on Sintering Phenomena. J. Appl. Phys. 21, 301 (1950).

[Her01] Herminghaus, S., Jacobs, K. und Seemann, R. The glass transition of thin polymer films: some questions, and a possible answer. Eur. Phys. J. E 5, 531 (2001).

[Hoo85] Hoover, W. G. Canonical dynamics: Equilibrium phase-space distributions. Phys. Rev. A 31, 1695 (1985).

[Hoo86] Hoover, W. G. Constant-pressure equations of motion. Phys. Rev. A 34, 2499 (1986).

[Hue94] Hues, S. M., Draper, C. F. und R. J. Colton, R. J. Measurement of nanomechanical properties of metals using the atomic force microscope. J. Vac. Sci. Technol. B 12, 2211 (1994).

[Isr92] Israelachvili, J. N. Intermolecular and surface forces. Academic Press London (1992).

[Jel48] Jellinek, H. H. G. Thermal degradation of polystyrene. Part I. J. Polym. Sci. 3, 850 (1948).

[Jel49a] Jellinek, H. H. G. Thermal degradation of polystyrene. Part II. J. Polym. Sci. 4, 1 (1949).

[Jel49b] Jellinek, H. H. G. Thermal degradation of polystyrene. Part III. J. Polym. Sci. 4, 13 (1949).

[Joh70] Johari, G. P. und Goldstein, M. Viscous liquids and the glass transition. II. Secondary relaxations in glasses of rigid molecules. J. Chem. Phys. 53, 2372 (1970).

[Kie31] Kiessig, H. Interferenz von Röntgenstrahlen an dünnen Schichten. Ann. d. Phys. 402, 769 (1931).

[KM05] Kopycinska-Müller, M. On the elastic properties of nanocrystalline materials and the determination of elastic properties on a nanoscale using the atomic force acoustic microscopy technique. Dissertation Universität des Saarlandes (2005). 
[KM08] Kopycinska-Müller, M., Caron, A., Hirsekorn, S., Rabe, U., Natter, H., Hempelmann, R., Birringer, R. und Arnold, W. Quantitative Evaluation of Elastic Properties of Nano-Crystalline Nickel Using Atomic Force Acoustic Microscopy. Zeit. f. Phys. Chem. 222, 471 (2008).

[Kni78] Knight, G. J. A comparison of the thermal stabilities of a siloxane substituted polycarbonate with a standard polycarbonate and a polydimethylsiloxane. Brit. Polym. J. 10, 187 (1978).

[Koh54] Kohlrausch, R. Theorie des elektrischen Rückstandes in der Leidener Flasche. Ann. d. Phys. 167, 56 (1854).

[Kop97] Kopf, A., Dünweg, B. und Paul, W. Dynamics of polymer isotope mixtures: Molecular dynamics simulation and Rouse model analysis. J. Chem. Phys. 107, 6945 (1997).

[Kra00] Kracke, B. und Damaschke, B. Measurement of nanohardness and nanoelasticity of thin gold films with scanning force microscope. Appl. Phys. Lett. 77, $361(2000)$.

[Kre88] Kremer, K., Grest, G. S. und Carmesin, I. Crossover from Rouse to Reptation Dynamics: A Molecular-Dynamics Simulation. Phys. Rev. Lett. 61, 566 (1988).

[Kre89] Kremer, K. Molekulardynamikmethoden. In 20. IFF-Ferienkurs, Computersimulationen in der Physik. Forschungszentrum Jülich GmbH (1989).

[Kre90] Kremer, K. und Grest, G. S. Dynamics of entangled linear polymer melts: A molecular-dynamics simulation. J. Chem. Phys. 92, 5057 (1990).

[Kru62] Kruse, P. W., McGlauchlin, L. D. und McQuistan, R. B. Elements of infrared technology: generation, transmission, and detection. Wiley, New York, London (1962).

[Kru99] Krug, J. und Rost, M. Linear theory of unstable growth on rough surfaces. Phys. Rev. B 60, 16334 (1999).

[Kub94] Kubono, A. und Okui, N. Polymer thin films prepared by vapor deposition. Prog. Polym. Sci. 19, 389 (1994).

[Lee64] Lee, L. H. Mechanisms of thermal degradation of phenolic condensation polymers. I. Studies on the thermal stability of polycarbonate. J. Polym. Sci. A 2, 2859 (1964).

[LeG00] LeGrand, D. G. und Bendler, J. T. (Hg.). Handbook of Polycarbonate Science and Technology. CRC Press (2000).

[Li99] Li, X.-G. und Huang, M.-R. Thermal degradation of bisphenol A polycarbonate by high-resolution thermogravimetry. Polym. Int. 48, 387 (1999). 
[Luf70] Luff, P. P. und White, M. The structure and properties of evaporated polyethylene thin films. Thin Sol. Films 6, 175 (1970).

[Lun00] Lunkenheimer, P., Schneider, U., Brand, R. und Loidl, A. Glassy dynamics. Contemp. Phys. 41, 15 (2000).

[Mad48] Madorsky, S. L. und Straus, S. High Vacuum Pyrolytic Fractionation of Polystyrene. Ind. Eng. Chem. 40, 848 (1948).

[Mad52] Madorsky, S. L. Rates of thermal degradation of polystyrene and polyethylene in a vacuum. J. Polym. Sci. 9, 133 (1952).

[Mad53] Madorsky, S. L. Rates and Activation Energies of Thermal Degradation of Styrene and Acrylate Polymers in. J. Polym. Sci. 9, 491 (1953).

[Mai91] Maivald, P., Butt, H. J., Gould, S. A. C., Prater, C. B., Drake, B., Gurley, J. A., Elings, V. B. und Hansma, P. K. Using force modulation to image surface elasticities with the atomic force microscope. Nanotechnol. 2, 103 (1991).

[Mak90] Maki, K. Evaporation of polyethylene powder for its thin film growth. Thin Sol. Films 188, 355 (1990).

[Man91] Mansfield, K. und Theodorou, D. Molecular dynamics simulation of a glassy polymer surface. Macromolecules 24, 6283 (1991).

[Mar99] Mark, J. E. Polymer data handbook. Oxford University Press (1999).

[May97] Mayr, S. G. Oberflächenrauigkeit von amorphen ZrAlCu-Schichten. Diplomarbeit Universität Augsburg (1997).

[May98] Mayr, S. G., Moske, M. und Samwer, K. Early stages in amorphous ZrAlCu film growth on HOPG. Europhys. Lett. 44, 465 (1998).

[May99] Mayr, S. G., Moske, M. und Samwer, K. Identification of key parameters by comparing experimental and simulated growth of vapor-deposited amorphous $Z_{65} A l_{7,5} C u_{27,5}$ films. Phys. Rev. B 60, 16950 (1999).

[May00] Mayr, S. G. Wachstum amorpher Schichten: Vergleich von Experiment und Simulation im Bereich Oberflächenrauigkeit und mechanische Spannungen. Dissertation Universität Göttingen (2000).

[May01] Mayr, S. G. und Averback, R. S. Surface Smoothing of Rough Amorphous Films by Irradiation-Induced Viscous Flow. Phys. Rev. Lett. 87, 196106 (2001).

[May09] Mayr, S. G. Relaxation kinetics and mechanical stability of metallic glasses and supercooled melts. Phys. Rev. B 79, 060201(R) (2009). 
Literaturverzeichnis

[McN91] McNeill, I. C. und Rincon, A. Degradation Studies of Some Polyesters and Polycarbonates VIII. Bisphenol A Polycarbonate. Polym. Degrad. Stab. 31, 163 (1991).

[McN93] McNeill, I. C. und Rincon, A. Thermal Degradation of Polycarbonates: Reaction Conditions and Reaction Mechanisms. Polym. Degrad. Stab. 39, 13 (1993).

[Mey04] Meyer, E., Hug, H. J. und Bennewitz, R. Scanning Probe Microscopy: The Lab on a Tip. Springer, Heidelberg 1. Aufl. (2004).

[Mit93] Mitlin, V. S. Dewetting of solid surface: analogy with spinodal decomposition. J. Coll. Interf. Sci. 156, 491 (1993).

[Mon87] Montaudo, G. und Puglisi, C. Thermal degradation mechanisms in condensation polymers. Dev. Polym. Degrad. 7, 35 (1987).

[Mon92] Montaudo, G. und Puglisi, C. Thermal-Decomposition Processes in Bisphenol-A Polycarbonate. Polym. Degrad. Stab. 37, 91 (1992).

[Muk06] Mukherji, D. und Müser, M. H. Possible explanation of the $\Lambda$-shape anomaly in polymer surface diffusion. Phys. Rev. E 74, 10601 (2006).

[Muk08] Mukherji, D., Bartels, G. und Müser, M. H. Scaling Laws of Single Polymer Dynamics near Attractive Surfaces. Phys. Rev. Lett. 100, 68301 (2008).

[Mul57] Mullins, W. M. Theory of Thermal Grooving. J. Appl. Phys. 28, 333 (1957).

[Mu159] Mullins, W. M. Flattening of a Nearly Plane Solid Surface due to Capillarity. J. Appl. Phys. 30, 77 (1959).

[Nak63] Nakamoto, K. Infrared Spectra of Inorganic and Coordination Compounds. Wiley, New York, London (1963).

[Nek73] Neki, K. und Geil, P. H. Morphology-property studies of amorphous polycarbonate. J. Macromol. Sci. B 8, 295 (1973).

[Nga88] Ngai, K. L. und Strom, U. High-frequency dielectric loss of Na $\beta$-alumina: Evidence for relaxation crossover. Phys. Rev. B 38, 10350 (1988).

[Nos84] Nosé, S. A molecular dynamics method for simulations in the canonical ensemble. Mol. Phys. 52, 255 (1984).

[Par54] Parrat, L. G. Surface Studies of Solids by Reflection of X-Rays. Phys. Rev. 95, 359 (1954).

[Pet06] Peter, S., Meyer, H. und Baschnagel, J. Thickness-dependent Reduction of the Glass Transition Temperature in Thin Polymer Films with a Free Surface. J. Polym. Sci. B 44, 2951 (2006). 
[Pet07] Peter, S., Meyer, H., Baschnagel, J. und Seemann, R. Slow dynamics and glass transition in simulated free-standing polymer films: a possible relation between global and local glass transition temperatures. J. Phys. Cond. Matt. 19, 205119 (2007).

[Pet08] Petersen, J. und Mayr, S. G. Dewetting of Ni and NiAg solid thin films and formation of nanowires on ripple patterned substrates. J. Appl. Phys. 103, 023520 (2008).

[Pli95] Plimpton, S. J. Fast parallel algorithms for short-range molecular dynamics. J. Comp. Phys. 117, 1 (1995). URL http://lammps.sandia.gov/.

[Pre02] Press, W. H., Teukolsky, S. A., Vetterling, W. T. und Flannery, B. P. Numerical Recipes in $C$, The Art of Scientific Computing. Cambridge University Press, Cambridge New York Port Chester Melbourne Sydney 2. Aufl. (2002).

[Qia07] Qian, H. J., Chen, L. J., Lu, Z. Y. und Li, Z. S. Surface Diffusion Dynamics of a Single Polymer Chain in Dilute Solution. Phys. Rev. Lett. 99, 68301 (2007).

[Rab94a] Rabe, U. und Arnold, W. Acoustic microscopy by atomic force microscopy. Appl. Phys. Lett. 64, 1493 (1994).

[Rab94b] Rabe, U. und Arnold, W. Atomic force microscopy at MHz frequencies. Ann. d. Phys. 506, 589 (1994).

[Rab96a] Rabe, U. Akustische Kraftmikroskopie im Ultraschallfrequenzbereich. Dissertation Universität des Saarlandes (1996).

[Rab96b] Rabe, U., Janser, K. und Arnold, W. Vibrations of free and surface-coupled atomic force microscope cantilevers: Theory and experiment. Rev. Sci. Inst. 67, 3281 (1996).

[Rab06] Rabe, U. Atomic Force Acoustic Microscopy. In Applied Scanning Methods II: Scanning Probe Microscopy Techniques. Springer (2006).

[Rad94] Radmacher, M., Fritz, M., Cleveland, J. P., Walters, D. A. und Hansma, P. K. Imaging adhesion forces and elasticity of lysozyme adsorbed on mica with the atomic force microscope. Langmuir 10, 3809 (1994).

[Rag00] Raghavan, D., Gu, X., Nguyen, T., VanLandingham, M. und Karim, A. Mapping polymer heterogeneity using atomic force microscopy phase imaging and nanoscale indentation. Macromolecules 33, 2573 (2000).

[Rai00] Raible, M., Mayr, S. G., Linz, S. J., Moske, M., Hänggi, P. und Samwer, K. Amorphous thin lm growth: Theory compared with experiment. Europhys. Lett. 50, 61 (2000).

[Rap04] Rapaport, D. C. The art of molecular dynamics simulation. Cambridge University Press (2004). 
[Ras82] Rasigni, G., Varnier, F., Rasigni, M. und Palmari, J. P. Autocovariance functions, root-mean-square-roughness height, and autocovariance length for rough deposits of copper, silver, and gold. Phys. Rev. B 25, 2315 (1982).

[Rat07a] Rath, S. und Port, H. Controlled Fabrication of Molecular Nano-Dot Patterns. J. Phys.: Conf. Ser. 61, 977 (2007).

[Rät07b] Rätzke, K., Zöllmer, V., Bartsch, A., Meyer, A. und Faupel, F. Diffusion in bulk-metallic glass-forming $\mathrm{Pd}-\mathrm{Cu}-\mathrm{Ni}-\mathrm{P}$ alloys: From the glass to the equilibrium melt. J. Non-Cryst. Sol. 353, 3285 (2007).

[Rei01] Reiter, G. Dewetting of Highly Elastic Thin Polymer Films. Phys. Rev. Lett. 87, 186101 (2001).

[Rei03] Reiter, G. Summary and conclusions: Progress in our understanding of instabilities in thin films. Eur. Phys. J. E 12, 465 (2003).

[Ric07] Richert, R. und Samwer, K. Enhanced diffusivity in supercooled liquids. New J. Phys. 9, 36 (2007).

[Rob01] Robertson, J. E. Thermal Degradation Studies of Polycarbonate. Phd thesis State University (2001).

[Rob02a] Robertson, J. E. und Ward, T. C. Development of a methodology to predict material properties from environmental exposure. I. Kinetic treatment. J. Polym. Sci. B 40, 794 (2002).

[Rob02b] Robertson, J. E. und Ward, T. C. Development of a methodology to predict material properties from environmental exposure. II. Structural features. J. Polym. Sci. B 40, 802 (2002).

[Rou53] Rouse, P. E. A Theory of the Linear Viscoelastic Properties of Dilute Solutions of Coiling Polymers. J. Chem. Phys. 21, 1272 (1953).

[Sah07] Sahin, O., Magonov, S. N., Su, C., Quate, C. F. und Solgaard, O. An atomic force microscope tip designed to measure time-varying nanomechanical forces. Nature Nanotech. 2, 507 (2007).

[Sch18] Scherrer, P. Bestimmung der Grösse und der inneren Struktur von Kolloidteilchen mittels Röntgenstrahlen. Nachr. Ges. Wiss. Göttingen 26, 98 (1918).

[Sch56] Schnell, H. Polycarbonate, eine gruppe neuartiger thermoplastischer kunststoffe. Angew. Chem. 68, 633 (1956).

[Sch64] Schnell, H. Chemistry and Physics of Polycarbonates. Wiley, New York (1964).

[Sch67] Schnell, H. und Bottenbruch, L. Über Polycarbonate. Naturwissenschaften 54, 306 (1967). 
[Sch97] Schnakenberg, J. Theorie und Simulation von Markov-Prozessen: Master-, Fokker-, Planck- und Langevin-Gleichungen. In 28. IFF-Ferienkurs, Dynamik und Strukturbildung in kondensierter Materie. Forschungszentrum Jülich GmbH (1997).

[Sch00] Schneider, U., Brand, R., Lunkenheimer, P. und Loidl, A. Excess wing in the dielectric loss of glass formers: A johari-goldstein beta relaxation? Phys. Rev. Lett. 84, 5560 (2000).

[Sch03] Schieber, J. D. Fluctuations in entanglements of polymer liquids. J. Chem. Phys. 118, 5162 (2003).

[Sch06] Scharf, T. Depositionsmechanismen, Struktur und mechanische Eigenschaften laserdeponierter Poly(methyl methacrylat)-Filme. Dissertation Universität Göttingen (2006).

[See01a] Seemann, R., Herminghaus, S. und Jacobs, K. Dewetting Patterns and Molecular Forces: A Reconciliation. Phys. Rev. Lett. 86, 5534 (2001).

[See01b] Seemann, R., Herminghaus, S. und Jacobs, K. Gaining control of pattern formation of dewetting liquid films. J. Phys. Cond. Mat. 13, 4925 (2001).

[Seo08] Seo, Y. und Jhe, W. Atomic force microscopy and spectroscopy. Rep. Prog. Phys. 71, 016101 (2008).

[Ser08] Serghei, A., Huth, H., Schick, C. und Kremer, F. Glassy dynamics in thin polymer layers having a free upper interface. Macromolecules 41, 3636 (2008).

[Sha93] Sharma, A. Relationship of thin film stability and morphology to macroscopic parameters of wetting in the apolar and polar systems. Langmuir 9, 861 (1993).

[Sou89] Souha, H., Viale, D., Weber, G. und Gillot, B. Effects of a silicon oxide layer on reactivity of silicon with copper (I) chloride. J. Mater. Sci. 24, 1767 (1989).

[Spe85] Sperling, L. H. Introduction to Physical Polymer Science. Wiley, New York (1985).

[Spr91] Springer, T. Einführung. In 22. IFF-Ferienkurs, Physik der Polymere. Forschungszentrum Jülich GmbH (1991).

[Sti95] Stillinger, F. H. A topographic view on supercooled liquids and glass formation. Science 267, 1935 (1995).

[Sto04] Stolz, M., Raiteri, R., Daniels, A. U., VanLandingham, M. R., Baschong, W. und Aebi, U. Dynamic Elastic Modulus of Porcine Articular Cartilage Determined at Two Different Levels of Tissue Organization by IndentationType Atomic Force Microscopy. Biophys. J. 86, 3269 (2004).

[Str04] Streng, C. Wachstumsanalyse amorpher dicker Schichten und Schichtsysteme. Dissertation Universität Göttingen (2004). 
[Str06] Streng, C., Samwer, K. und Mayr, S. G. Surface smoothing and pattern formation in the transient region of compositionally modulated amorphous metallic alloy films: Experiment versus theory. Phys. Rev. B 73, 104107 (2006).

[Suk00] Sukhishvili, S. A., Chen, Y., Müller, J. D., Gratton, E., Schweizer, K. S. und Granick, S. Diffusion of a polymer pancake. Nature 406, 146 (2000).

[Süs05] Süske, E. Charakteristische Eigenschaften laserdeponierter Poly(methylmethacrylat)-Filme. Dissertation Universität Göttingen (2005).

[Tam96] Tamayo, J. und Garcia, R. Deformation, contact time, and phase contrast in tapping mode scanning force microscopy. Langmuir 12, 4430 (1996).

[Ton94] Tong, W. M. und Williams, R. S. Kinetics of Surface Growth: Phenomenology, Scaling, and Mechanisms of Smoothening and Roughening. Annu. Rev. Phys. Chem. 45, 401 (1994).

[Tor00] Torres, J. A., Nealey, P. F. und de Pablo, J. J. Molecular Simulation of Ultrathin Polymeric Films near the Glass Transition. Phys. Rev. Lett. 85, 3221 (2000).

[Tur58] Turnbull, D. und Cohen, M. H. Concerning Reconstructive Transformation and Formation of Glass. J. Chem. Phys. 29, 1049 (1958).

[Ued98] Ueda, Y., Matsushita, M., Morimoto, S., Ping Ni, J., Suzuki, H. und Mashiko, S. Structure and crystal growth of low molecular weight polyethylene vapordeposited on polymer friction-transferred layers. Thin Sol. Films 331, 216 (1998).

[Var02] Varnik, F., Baschnagel, J. und Binder, K. Reduction of the glass transition temperature in polymer films: A molecular-dynamics study. Phys. Rev. E 65, 21507 (2002).

[Vau02] Vauth, S. Experimente und Simulationen zum Wachstum amorpher Aufdampfschichten bei schräger Teilchendeposition. Diplomarbeit Universität Göttingen (2002).

[Vau05] Vauth, S. und Mayr, S. G. Atomic dynamics in molecular dynamics simulations of glassy CuTi thin films. Appl. Phys. Lett. 86, 061913 (2005).

[Vau07a] Vauth, S. Glättungsmechanismen beim Ionenbeschuss rauer amorpher Oberflächen. Dissertation Universität Göttingen (2007).

[Vau07b] Vauth, S. und Mayr, S. G. Relevance of surface viscous flow, surface diffusion, and ballistic effects in keV ion smoothing of amorphous surfaces. Phys. Rev. B 75, 224107 (2007).

[Vau08] Vauth, S. und Mayr, S. G. Ion bombardment induced smoothing of amorphous metallic surfaces: Experiments versus computer simulations. Phys. Rev. B 77, 155406 (2008). 
[Ver67] Verlet, L. Computer Ëxperimentsön Classical Fluids. I. Thermodynamical Properties of Lennard-Jones Molecules. Phys. Rev. A 159, 98 (1967).

[Vil91] Villain, J. Continuum models of crystal growth from atomic beams with and without desorption. J. Phys. I (France) I, 19 (1991).

[Vre05] Vree, C. Experimente und Simulationen zum Wachstum dünner Polymerfilme. Diplomarbeit Universität Göttingen (2005).

[Vre06] Vree, C. und Mayr, S. G. Kinetic roughening during vapor deposition of polymer films: A Monte Carlo study. J. Appl. Phys. 100, 013511 (2006).

[Vre08] Vree, C. und Mayr, S. G. Structure and surface morphology of vapor deposited polycarbonate thin films. J. Appl. Phys. 104, 083517 (2008).

[Vre09a] Vree, C. und Mayr, S. G. Dynamics and diffusive - conformational coupling in polymer bulk samples and surfaces: a molecular dynamics study. Phys. Rev. B submitted (2009).

[Vre09b] Vree, C. und Mayr, S. G. Pattern formation during vapor deposition of organic films on inorganic substrates-continuum modeling vs experiments. Appl. Phys. Lett. 94, 093110 (2009).

[Vri66] Vrij, A. Possible mechanism for the spontaneous rupture of thin, free liquid films. Disc. Farad. Soc. 42, 23 (1966).

[Was05] Waser, R. Nanoelectronics and information technology. Wiley-VCH, Weinheim (2005).

[Wei03] Weitze, M. D. Das Rasterkraftmikroskop. Deutsches Museum, Naturwissenschafts- und Technikgeschichte (2003).

[Wil70] Williams, T. Gel Permeation Chromatography: A Review. J. Mater. Sci. 5, 811 (1970).

[Win06] Winkler, R. G. Molecular Dynamics Simulation. In 3\%. IFF-Ferienkurs, Computational Condensed Matter Physics. Forschungszentrum Jülich GmbH (2006).

[Wol90] Wolf, D. E. und Villain, J. Growth with surface diffusion. Europhys. Lett. 13, 389 (1990).

[Wol96] Wolfgardt, M. und Binder, K. On the equation of state for thermal polymer solutions and melts with attractive interaction. Macromol. Theory Simul. 5, 699 (1996).

[Xie98] Xie, R., Karim, A., Douglas, J. F., Han, C. C. und Weiss, R. A. Spinodal Dewetting of Thin Polymer Films. Phys. Rev. Lett. 81, 1251 (1998). 
[Yak61] Yakubovich, Y. A., Gordon, G. Y., Maslennikova, L. I., Grobman, E. M., Tret'Yakova, K. I. und Kokoreva, N. I. Investigations of the chemical conversions of polycarbonates. J. Polym. Sci. 55, 251 (1961).

[Yam96] Yamanaka, K. und Nakano, S. Ultrasonic Atomic Force Microscope with Overtone Excitation of Cantilever. Jpn. J. Appl. Phys. 35, 3787 (1996).

[Yan06] Yang, H., Lu, Z. Y., Li, Z. S. und Sun, C. C. A molecular-dynamics simulation study of diffusion of a single model carbonic chain on a graphite (001) surface. J. Mol. Model. 12, 432 (2006).

[Yos04] Yoshimoto, K., Jain, T. S., Workum, K. V., Nealey, P. F. und de Pablo, J. J. Mechanical Heterogeneities in Model Polymer Glasses at Small Length Scales. Phys. Rev. Lett. 93, 175501 (2004).

[Zap01] Zaporojtchenko, V., Strunskus, T., Erichsen, J. und Faupel, F. Embedding of Noble Metal Nanoclusters into Polymers as a Potential Probe of the Surface Glass Transition. Macromolecules 34, 1125 (2001).

[Zar91] Zarzycki, J. Glasses and the vitreous state. Cambridge University Press, New York, Port Chester, Melbourne, Sydney (1991).

[Zav08] Zavala, G. Atomic force microscopy, a tool for characterization, synthesis and chemical processes. Coll. Polym. Sci. 286, 85 (2008).

[Zha00] Zhao, Y.-P., Fortin, J. B., Bonvallet, G., Wang, G.-C. und Lu, T.-M. Kinetic Roughening in Polymer Film Growth by Vapor Deposition. Phys. Rev. Lett. 85, 3229 (2000).

[Zho93] Zhong, Q., Inniss, D., Kjoller, K. und Elings, V. B. Fractured polymer/silica fiber surface studied by tapping mode atomic force microscopy. Surf. Sci. Lett. 290, L688 (1993).

[Zin06] Zink, M., Samwer, K., Johnson, W. L. und Mayr, S. G. Validity of temperature and time equivalence in metallic glasses during shear deformation. Phys. Rev. B 74, 12201 (2006). 


\section{Danksagung}

An dieser Stelle möchte ich mich bei all denjenigen herzlich bedanken, die direkt oder indirekt zum Entstehen dieser Arbeit beigetragen haben.

Herrn Prof. Dr. Stefan G. Mayr danke ich für die Betreuung meiner Arbeit. Er hat es mir ermöglicht, dieses interessante Thema zu bearbeiten. Sein Engagement und Interesse sowie die damit verbundenen hilfreichen Diskussionen, Tipps und Anregungen haben wesentlich zum Gelingen der Arbeit beigetragen.

Herrn Prof. Dr. Stephan Herminghaus möchte ich für die Übernahme des Korreferats danken.

Herrn Prof. Dr. Walter Arnold danke ich für die Unterstützung und das Interesse am Fortschritt des AFAM Aufbaus. In diesem Zusammenhang möchte ich auch Hannes Wagner für die gute Zusammenarbeit, die Programmierung der AFAM Auswertung und sein Engagement bei der Weiterentwicklung und der Fortführung der AFAM Messungen danken.

Herrn Dr. Björn Steisel und Herrn Dr. Fabian Günzler aus der Arbeitsgruppe von Herrn Prof. Dr. Michael Buback bin ich für die Durchführung der SEC und Massenspektrometrie Messungen sowie deren Deutung zu Dank verpflichtet.

Der Polymergruppe um Herrn Prof. Dr. Hans-Ulrich Krebs möchte ich für die Zusammenarbeit und die vielen wichtigen Diskussionen danken.

Ohne die Unterstützung unserer Techniker Carsten Mahn, Uta Bete und Katrin Gehrke und der Crew der feinmechanischen Werkstatt wäre diese Arbeit wohl nicht möglich gewesen. Dafür danke ich ihnen sehr.

Ansonsten bleibt mir, mich bei meiner Arbeitsgruppe und allen anderen Mitgliedern der I. Physik für das tolle Arbeitsklima zu bedanken. Die Arbeit im Institut hat mir auch wegen der guten Stimmung bei allen Mitarbeitern stets viel Spaß gemacht. Hervorzuheben sind dabei Dr. Jan Petersen und meine ehemaligen Zimmerkollegen Dr. Jörg Hachenberg und Dr. Sebastian Vauth, die mir von Beginn an sowohl bei physikalischen als auch bei nicht fachlichen Fragen weitergeholfen haben. Meinen aktuellen Sitznachbarn Melanie Schneider und Christoph Bergmann danke ich für die weiterhin angenehme Atmosphäre. 
Mein besonderer Dank gilt Dennis Bedorf und Tobias Edler, die in vielen Diskussionen zum Fortschritt der Arbeit beigetragen haben. Beide hatten immer ein offenes Ohr für mich und waren außerdem zum Korrekturlesen dieser Arbeit und der anderen Veröffentlichungen bereit. Und ohne Dennis wäre der tägliche Gang zur Mensa nur halb so lustig.

Ganz besonderen Dank haben meine Eltern verdient, die mich immer und in allen Lebenslagen unterstützt haben.

Und zum Schluss möchte ich meiner Freundin Sarah dafür danken, dass sie immer für mich da war und ist.

„Denn das Glück, geliebt zu werden, ist das höchste Glück auf Erden.“

(Johann Gottfried von Herder) 


\section{Publikationen}

2006: C. Vree and S. G. Mayr:

Kinetic roughening during vapor deposition of polymer films:

A Monte Carlo study,

J. Appl. Phys. 100, 013511 (2006)

2008: C. Vree and S. G. Mayr:

Structure and surface morphology of vapor deposited polycarbonate thin films,

J. Appl. Phys. 104, 083517 (2008)

2009: C. Vree and S. G. Mayr:

Pattern formation during vapor deposition of organic films on inorganic substrates - continuum modelling vs. experiments, Appl. Phys. Lett. 94, 093110 (2009)

C. Vree and S. G. Mayr:

Dynamics and diffusive - conformational coupling in polymer bulk samples and surfaces: a molecular dynamics study,

Phys. Rev. B , submitted (2009) 



\section{Lebenslauf}

\section{Persönliche Daten}

$\begin{array}{ll}\text { Name: } & \text { Christian Vree } \\ \text { Geburtsdatum: } & \text { 12. November } 1979 \\ \text { Geburtsort: } & \text { Wolfenbüttel } \\ \text { Staatsangehörigkeit: } & \text { Deutsch }\end{array}$

\section{Schulausbildung}

$\begin{array}{ll}08.1986-07.1990 & \text { Grundschule Groß Elbe } \\ 08.1990-07.1992 & \text { Orientierungsstufe Baddeckenstedt } \\ 08.1992-06.1999 & \text { Gymnasium Salzgitter Bad } \\ \text { 28.06.1999 } & \text { Abitur }\end{array}$

\section{Grundwehrdienst}

$07.1999-04.2000 \quad$ Grundwehrdienst, Heeresflieger in Celle

\section{Hochschulausbildung}

10.2000 - $07.2005 \quad$ Studium der Physik an der Georg-August-Universität Göttingen 01.2004 - 07.2005 Diplomarbeit im Bereich Festkörperphysik bei Herrn Prof. Dr. S. G. Mayr mit dem Thema: „Experimente und Simulationen zum Wachstum dünner Polymerfilme“

08.07.2005 Diplom in Physik (Note: Sehr gut)

seit 10.2005 Promotionsstudium im Fach Physik an der Georg-AugustUniversität Göttingen

seit 09.2005

Wissenschaftlicher Mitarbeiter am I. Physikalischen Institut der Georg-August-Universität Göttingen und Anfertigung der vorliegenden Dissertation 